\title{
Review of Simulation Techniques for Aquifer Thermal Energy Storage (ATES)
}

\section{March 1981}

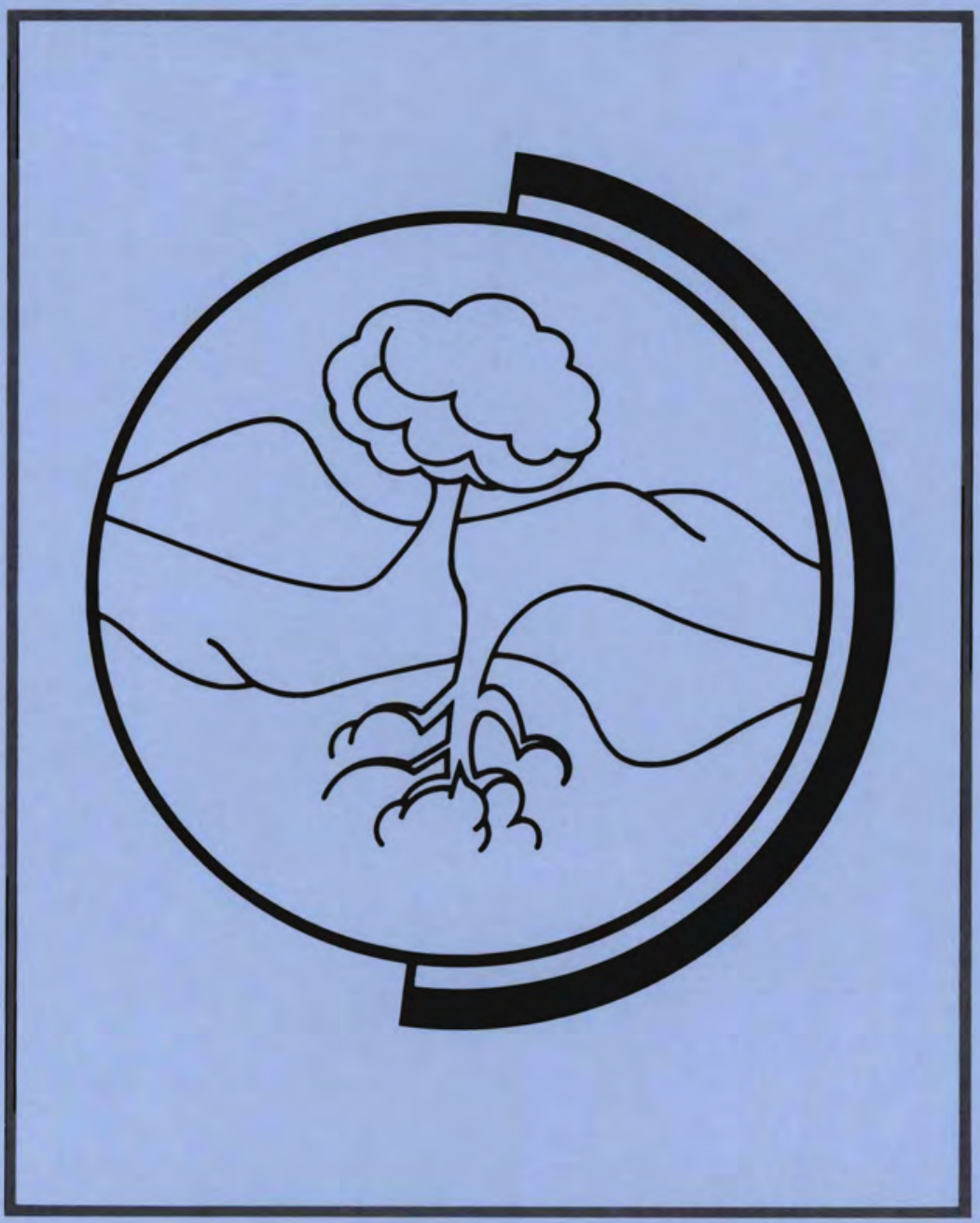

Prepared for Pacific Northwest Laboratory Seasonal Thermal Energy Storage Program under Special Agreement B-67764-A-O

Pacific Northwest Laboratory Operated for the U.S. Department of Energy by Battelle Memorial Institute 
NOTICE

This report was prepared as an account of work sponsored by the United States Government. Neither the United States nor the Department of Energy, nor any of their employees, nor any of their contractors, subcontractors, or their employees, makes any warranty, express or implied, or assumes any legal liability or responsibility for the accuracy, completeness or usefulness of any information, apparatus. product or process disclosed, or represents that its use would not infringe privately owned rights.

The views, opinions and conclusions contained in this report are those of the contractor and do not necessarily represent those of the United States Government or the United States Department of Energy.

\author{
PACIFIC NORTHWEST LABORATORY \\ operated by \\ BATTELLE \\ for the \\ UNITED STATES DEPARTMENT OF ENERGY \\ Under Contract DE-AC06-76RLO 1830
}

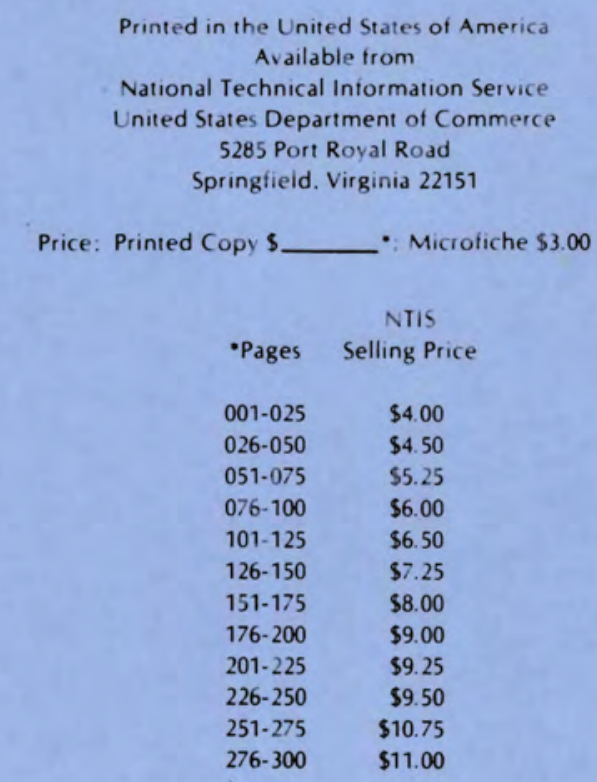


REVIEW OF SIMULATION TECHNIQUES

FOR AQUIFER THERMAL ENERGY STORAGE (ATES)

J. W. Mercer

C. R. Faust

W. J. Miller

GeoTrans, Inc.

Herndon, VA

F. J. Pearson, Jr.

INTERA Environmental Consultants, Inc. Houston, TX

March 1981

Prepared for Pacific Northwest Laboratory Seasonal Thermal Energy Storage Program under Special Agreement B-B2424-A-0 under U.S. Department of Energy Contract DE-AC06-76RLO 1830

Pacific Northwest Laboratory Richland, Washington 99352 



\section{ACKNOWLEDGMENTS}

This report was prepared by James Mercer, Charles Faust, and William Miller of GeoTrans, Inc. and by F.J. Pearson, Jr. of INTERA, Environmental Consultants, Inc. under a subcontract issued by Pacific Northwest Laboratory (No. B-B2424-A-0). Charles Kincaid of PNL served as technical contract officer. We gratefully acknowledge the help of Carol Roberts and Virginia van Gessel in preparing the manuscript. We also wish to thank Jack Guswa of the U.S. Geological Survey for information on the numerical simulation aspects of the ongoing demonstration program at the University of Minnesota. Information on the Texas A\&M experiment was received from Don Reddell (of the Texas A\&M faculty) and is gratefully acknowledged. 


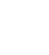




\section{SUMMARY}

The storage of thermal energy, in aquifers has recently received considerable attention as a means to conserve and more efficiently use energy supplies. The analysis of aquifer thermal energy storage (ATES) systems will rely on the results from mathematical and geochemical models. The purpose of this report, therefore, is to review and evaluate the state-of-the-art models relevant to ATES. These models describe important processes active in ATES including ground-water flow, heat transport (heat flow), solute transport (movement of contaminants), and geochemical reactions.

In general, available models of the saturated ground-water environmerit are adequate to address most concerns associated with ATES; that is, design, operation, and environmental assessment. The validation of presently available codes against a range of representative ATES applications should precede further development of aquifer models. The creation of comprehensive data bases from a variety of ATES applications is necessary for this validation. Furthermore, basic data deficiencies must be remedied for geochemical reaction and biological processes before models of these processes can be applied to ATES problems.

In those cases where models are not adequate, development should be preceded by efforts to identify significant physical phenomena and relate model parameters to measurable quantities. Model development can then proceed with the expectation of an adequate data base existing for the model's eventual use. A notable example of modeling deficiency lies in the coupled transport of fluid and energy within unsaturated sediments overlying unconfined aquifers. More complete understanding of these transport processes is required before the potential for ATES in unconfined aquifers can be evaluated.

Review of model applications to ATES shows that the major emphasis has been on generic sensitivity analysis and site characterization. Generic sensitivity analysis (determining how various parameters affect the success of ATES) has provided valuable insight into understanding important processes. Additional generic studies, however, will not be as useful as 
(nor should they be substituted for) the more important model applications to specific sites. The results from generic studies show how ideal systems behave. Applications to specific sites will demonstrate the sufficiency of the idealized models and/or indicate research needs.

Assuming that models are applied appropriately, the primary limitation on model calculations is the data base used to construct the mode1. Numerical transport models are limited by the uncertainty of subsurface data and the lack of long-term historical data for calibration. Geochemical models are limited by the lack of thermodynamic data for the temperature ranges applicable to ATES. Model applications undertaken with data collection activities on ATES sites should provide the most important contributions to the understanding and utilization of ATES. Therefore, the primary conclusion of this review is that model application to field sites in conjunction with data collection activities is essential to the development of this technology. 


\section{TABLE OF CONTENTS}

ACKNOWLEDGMENTS

SUMMARY

TABLE OF CONTENTS vi i

LIST OF FIGURES. . . . . . . . . . . . . . . . . . . . . . . $x V$

LIST OF TABLES . . . . . . . . . . . . . . . . . . . . xvi . . . . . . . . .

INTRODUCTION . . . . . . . . . . . . . . . . . . . . 1

PURPOSE AND SCOPE ........................ 1

LITERATURE REVIEW . . . . . . . . . . . . . . . . . 4

CONCLUSIONS AND RECOMMENDATIONS .................. 5

PHYSICAL AND CHEMICAL PROCESSES . . . . . . . . . . . . . . 9

FLUID FLOW ......................... 9

HEAT TRANSPORT . . . . . . . . . . . . . . . . . 13

Conduction-Dispersion .............. 13

Convection ....................... 14

Heat Capacity .................... 14

SOLUTE TRANSPORT . . . . . . . . . . . . . . . 15

Dispersion .......................... 15

Source Term Properties............... 20

GEOCHEMISTRY . . . . . . . . . . . . . . 20

Corrosion Chemistry ................ 21

Mineral Deposition .................. 21

Scaling................... . . 22

Calcium Carbonate................ 22

Silica.......................... 23

Mixing of Fluids . . . . . . . . . . . . . . . 24 
WATER-ROCK INTERACTIONS . . . . . . . . . . . . 24

Dispersion of Interstitial Clay .......... 25

Particulate Plugging. . . . . . . . . . . 26

BIOLOGICAL FACTORS ................ 26

WELL FACTORS . . . . . . . . . . . . . . . . 28

Well-Bore Heat Loss . . . . . . . . . . . . 28

Well Design and Location............. . 28

MATHEMATICAL DESCRIPTION . . . . . . . . . . . . . 30

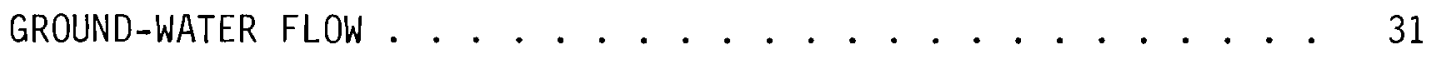

General Equation . . . . . . . . . . . 31

Pressure Formulation .......... 31

Hydraulic Head Formulation . . . . . . . . 34

Variations of the General Equation ......... 36

Reduced Cartesian Equations .......... 36

Radial Equation ............ . 37

Isothermal Equations . . . . . . . . 38

HEAT TRANSPORT . . . . . . . . . . . . . . . . . 39

General Equation ............ 39

Variations of the General Equation.......... 44

Reduced Cartesian Equations ........... . 44

Radial Equation ............. . 45

SOLUTE TRANSPORT ............................. 45

BOUNDARY AND INITIAL CONDITIONS . . . . . . . . . . 48

FLOW IN FRACTURED, FISSURED, OR CAVERNOUS MEDIA . . . . . 51 


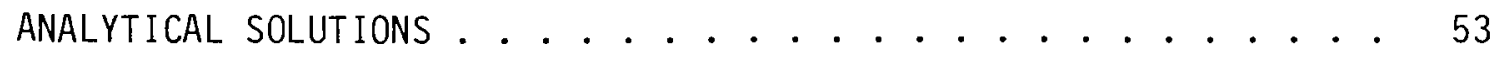

RECHARGE-DISCHARGE PAIR SOLUTION . . . . . . . . . 54

Problem ........................ 54

Assumptions ....................... 54

Solution ...................... 54

Possible Usage .................... 58

LAUWERIER SOLUTION ................................ 58

Problem ...................... 58

Assumptions. .................... 59

Solution ................... 60

Possible Usage ................... 60

GRINGARTEN ET AL. SOLUTION . . . . . . . . . . 60

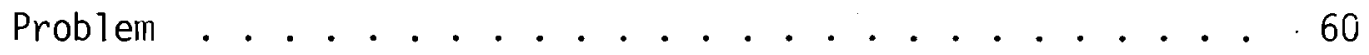

Assumptions ..................... 61

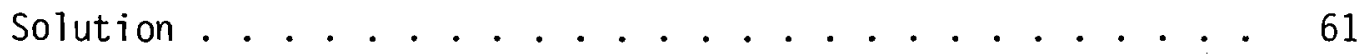

Possible Usage ................... 61

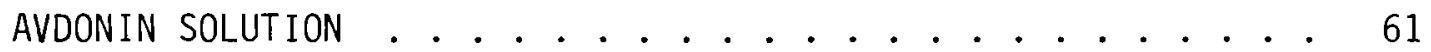

Problem .................... 61

Assumptions ..................... 62

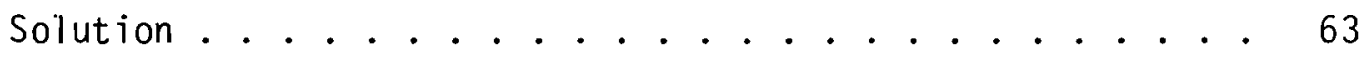

Possible Usage .................. . . 63

ONE-DIMENSIONAL HEAT CONDUCTION .................... 64

Problem ........................ 64

Assumptions ..................... 65

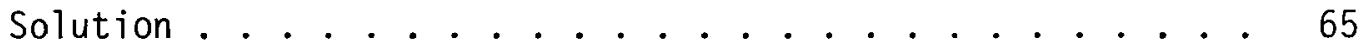

Possible Usage . . . . . . . . . . . . . 65 
GRINGARTEN AND SAUTY SOLUTION . . . . . . . . . . . . . 65

Problem ...................... 65

Assumptions ................... 66

Solution ......................... 66

Possible Usage . . . . . . . . . . . . . . . . . 68

TILTING FRONT SOLUTION . . . . . . . . . . . . . . . 68

Problem .................... . . 68

Assumptions ................... . . 68

Solution . . . . . . . . . . . . . . . 68

Possible Usage . . . . . . . . . . . . . . . 70

NUMERICAL SOLUTIONS . . . . . . . . . . . . . . . . 71

CCC........................... 73

Governing Equations .............. . . 73

Assumptions . . . . . . . . . . . . . . . 77

Solution . . . . . . . . . . . . . . 77

Applications . . . . . . . . . . . . . . . 77

SWIP . . . . . . . . . . . . . . . . . . 84

Governing Equations . . . . . . . . . . . . 84

Assumptions .................... . . . 84

Solution . . . . . . . . . . . . . . . . 85

Applications . . . . . . . . . . . . . . 87

GEOTHER ...................... 87

Governing Equations .............. 87

Assumptions . . . . . . . . . . . . . . . 90

Solution . . . . . . . . . . . . . . . 91

Applications . . . . . . . . . . . . . . . . 91 
TEXAS A\&M FINITE ELEMENT MODEL . . . . . . . . . . . . . 94

Governing Equations ................ 94

Assumptions . . . . . . . . . . . . . . . 94

Solution . . . . . . . . . . . . . . . . 94

Applications ............................... 95

GEOCHEMICAL MODELS . . . . . . . . . . . . . . . . 97

AQUEOUS SOLUTION MODELS . . . . . . . . . . . . . . . . 99

Conceptual Models .................... 99

Ion-Association Model . . . . . . . . . . . 100

Mixed-Electrolyte Model . . . . . . . . . . 101

Ion-Association Models . . . . . . . . . . . . 102

Governing Equations . . . . . . . . . . 102

Solution Techniques .................... 106

Applications .......................... 110

GEOCHEMICAL REACTION MODELS . . . . . . . . . . . . 112

Mass Transfer Models . . . . . . . . . . . 112

Governing Equations ............... 113

Solution Techniques . . . . . . . . . . . . 114

Applications ................... . . 114

Reaction Path Models ............... 115

Governing Equations.............. . . 115

Solution Techniques . . . . . . . . . . . 116

Applications .................... 117 
MODEL APPLICATIONS ............................... 117

Effects of Changing Temperature/Pressure . . . . . 117 Gas Solubility ................ 118

Mineral Solubility ............ . 119

Effects of Mixing Injected and Formation Waters . . . . 121

Effect of Injected Water On Formation Mineral Solubilities . 122 MODEL LIMITATIONS . . . . . . . . . . . . . . . 126

Limitations in Model Assumptions . . . . . . . . . 126

Limitations to Model Use . . . . . . . . . . 128 APPLICATIONS OF TRANSPORT MODELS . . . . . . . . . . 130 REVIEW OF FIELD APPLICATIONS . . . . . . . . . . 130

Krefeld, Germany ............. 132

Location ................... 132

Hydrogeology . . . . . . . . . . . 132

Description of Field Operations . . . . . . . 132

Discussion ................... 133

Neuchatel, Switzerland ........... 135

Location . . . . . . . . . . . . . . 135

Hydrogeology ..................... 135

Description of Field Operations .............. 135

Discussion . . . . . . . . . . . . . . 136

Mobile, Alabama .............. 137

Location . . . . . . . . . . . 137

Hydrogeology . . . . . . . . . . . . 137

Description of Field Operations . . . . . . . 138

Discussion ...................... 138 
Bonnaud, France .............. 143

Location . . . . . . . . . . 143

Hydrogeology . . . . . . . . . . 143

Description of Field Operations .......... 143

Discussion .................... 144

Gard, France . . . . . . . . . . . . 144

Location . . . . . . . . . . 144

Hydrogeology ................... 145

Description of Field Operations . . . . . . . 145

Discussion ............. 146

Yamagata Basin, Japan ............ 147

Location . . . . . . . . . . . . 147

Hydrogeology . . . . . . . . . . . 147

Description of Field Operations . . . . . . 147

Discussion ................ 148

College Station, Texas ............. 150

Location . . . . . . . . 150

Hydrogeology . . . . . . . . . . 150

Description of Field Operations ......... 150

Discussion ..................... 151

Minneapolis-St. Paul, Minnesota . . . . . . . 152

Location ............ . . 152

Hydrogeology . . . . . . . . . . 152

Description of Field Operations . . . . . . . . 152 
Stony Brook, New York ............. 153

Location . . . . . . . . . 153

Hydrogeology ........... . . 153

Description of Field Operations ........ . 153

Bethel, Alaska ............... 154

Location ............ . . 154

Hydrogeology ................ . . 154

Description of Field Operations . . . . . . . . 154

SITE-SPECIFIC CONSIDERATIONS . . . . . . . . . . . 155

AQUIFER CHARACTERISTICS . . . . . . . . . . . . 155

Shallow Water-Table Aquifers .......... 155

Deep Water-Table Aquifers . . . . . . . . . . 157

Thin Confined Aquifers . . . . . . . . . 157

Thick Confined Aquifers . . . . . . . . . . 157

Heterogeneous Aquifers . . . . . . . . 158

Fissured or Cavernous Aquifers . . . . . . . . . 158

FLUID AND ROCK PROPERTIES . . . . . . . . . . . 160

BOUNDARY AND INITIAL CONDITIONS ....................... 163

USES AND LIMITATIONS OF TRANSPORT MODELS . . . . . . . . . . 164

SITE-SPECIFIC MODEL USE . . . . . . . . . . . . . 164

MODEL LIMITATIONS . . . . . . . . . . . . . . . . 167

REFERENCES ........................ 170

APPENDIX A - EQUATION DEVELOPMENT ............. A-1

APPENDIX B - NUMERICAL APPROXIMATIONS .......... B-1 


\section{LIST OF FIGURES}

Figure

Legend

Page

1

2

3

4

5

6

7

8

9

10

Schematic of Aquifer Thermal Energy Storage

(after Reilly 1980)............ 2

Idealized injection-recovery problem

iliustrating concentration front inovement between injection and recovery we.lis. With convection on $7 y$, concentration moves out as a sharp front. With dispersion, the front is smeared (from Mercer and Faust 1980) . . . . . 18

A) Physical situation of an instantaneous uniform concentration source in an aquifer. B) Typical discrepancy between observed and predicted concentration distributions for some later time $t_{1}$ (from Mercer and Faust 1980). . .

Major components of the ground-water flow equation (from Mercer and Faust 1980) . . . . . 33

Major components of the heat transport equation (from Mercer and Faust 1980) . . . . . . .

Components of the solute transport equation (from Mercer and Faust 1980) . . . . . . . .

Section showing water levels in a rechargedischarge pair (doublet) . . . . . . . .

Generalized model development by finitedifference and finite element methods (from Faust and Mercer 1980)

The recovery factor as a function of aquifer thickness and storage radius. The latter is defined as the radius reached by the storage temperature front at the end of injection period (from Tsang 1980) . . . . . . . . 80

The recovery factor as a function of storage volume, implicitly given by $\mathrm{R}^{2} \mathrm{thH}$ for different values of aquifer thickness $H$ (from Tsang 1980) . . 


\section{LIST OF FIGURES}

Figure

11

12

13

14

15

16

17

18

A-1

$A-2$

Legend

Page

The recovery factor as a function of aspect ratio, storage radius over aquifer thickness (Rth/H), for different aquifer and aquitard thermal conductivities ( $\lambda_{a}$ and $\lambda_{c}$ )

(from Tsang 1980).

The recovery factor as a function of cycle time periods $\left(t_{\text {cycle }}\right)$ (from Tsang 1980). . . . . .

Avdonin analytic solution (solid line) for a time of $3.75 \times 10^{8} \mathrm{~s}$ and a reservoir length of $1187.5 \mathrm{~m}$ (distance measured from injection source is indicated as dimensionless). Also shown are finite-difference solutions for midpoint and upstream weighting (from Faust and Mercer 1979b).

Isotherm illustration in longitudinal section parallel to the flow of the ground water

A. Field experiment; B. Calculation model (from Werner and Kley 1977) . . . . . . . . .

Comparison of simulated and observed temperature history at selected depths in Wel1 1 (from Papadopulos and Larson 1978). . . . .

Comparison of simulated and observed temperatures of the recovered water (from Papadopulos and Larson 1978) . . . . . . .

Example results showing the effect of

fracture zone thickness (b) on temperatures

in fracture and percent of heat retained in

the fracture zone ................

Flow chart showing model use (from Mercer and Faust 1980)

Variation in bulk density as a function of the average volume, with inserts (from Mercer and Faust 1980)

Volume element of a porous material

showing flow across all faces

(from Mercer and Faust 1980) 


\section{LIST OF FIGURES}

Figure

Legend

Page

B-1 Grids showing mesh-centered nodes and block-centered nodes (from Faust and Mercer 1980) . . . . . . B B-2

B-2 Four by five block-centered grid used in difference equation development $(A)$ and typical connection (B) for node $(i, j)$ (from Faust and Mercer 1980) . . . . . . B B-3

B-3 Polygon geometry (after Thomas 1973). . . . B-5

B-4 Finite element configuration showing typical node and element (from Faust and Mercer 1980) . . B-8

B-5 Surface described by linear basis function for a triangular element (from Faust and Mercer 1980) 


\section{LIST OF TABLES}

$\underline{\text { Table }}$

Legend

Page

1

2

3

4

5

6

7

8

10

11

12

13

14

15

16

Processes with dependent variables and application variations ........ 10

Regional dispersivities determined from areal model studies (after Anderson 1979) . . . . . . . . . 16

Boundary conditions for ground-water flow, heat transport, and solute transport .. . 49 Summary of analytical solutions ....... 55 Modeling studies in ATES (extended from Tsang et al. 1980a) ..... 74

Summary of model characteristics and options for CCC code . . . . . . . . 78

Summary of numerical diffusion and stability (from INTERCOMP 1979) . . . . . 86

Summary of model characteristics and options for SWIP code . . . . . . . . . 88

Summary of model characteristics and options for GEOTHER code . . . . . . 92 Summary of model characteristics and options for the Texas A\&M code . . . . . 96 Partial summary of geochemical models . . . . . . 107 Data from ATES field experiments (extended and modified from Tsang et al. 1980a) . 131 Parameters for heat-transport model . . . . . 139 Summary of injection/production information . . 149 Desirable characteristics of an ATES project and corresponding favorable and unfavorable aquifer conditions ..... 156 Reported parameters used for field applications . 162 


\section{INTRODUCTION}

Methods for storing heat energy are needed to maximize the benefits of some alternative energy supplies and to minimize the waste associated with conventional energy utilization. One alternative energy supply that is being investigated is solar energy, which may be used to produce heat. Energy conservation is concerned with using available energy more effectively, i.e., minimizing waste. Usually this waste is in the form of heat and is associated with (Reffstrup 1979): (1) power plants where electricity and heat are produced in co-generation, (2) large industrial plants where heat is used in industrial processes, and (3) refuse disposal plants. The most probable use of this waste heat is for space heating. Unfortunately, the seasonal production of waste heat or solar energy in the summer and the demand for space heating do not generally coincide, with the demand for space heating being highly variable depending on the season. According to Reffstrup (1979), the seasonal variation in the demand for heat is the main obstacle for more general usage of waste heat for space heating purposes and for a large-scale use of energy sources such as solar energy.

\section{PURPOSE AND SCOPE}

A solution to this problem is the uncoupling of waste heat and solar energy production and heat demand through large-scale seasonal storage of hot water or seasonal thermal energy storage (STES). One method of storage that has been suggested is to pump the hot water into ground-water aquifers (Kazmann 1971) or aquifer thermal energy storage (ATES). This concept has been extended to include the storage of cold water. Thus, in general, ATES involves injecting either hot or cold fluid into an aquifer, where it is stored until it is needed for space heating or cooling, when it is pumped out. This concept is demonstrated in Figure 1, where the shaded area in the aquifer represents the injected fluid.

The well system demonstrated in Figure 1 is called a well doublet. Using conventional drilling and well-completion techniques, two separate wells are constructed in an aquifer. Water is withdrawn from one well, 


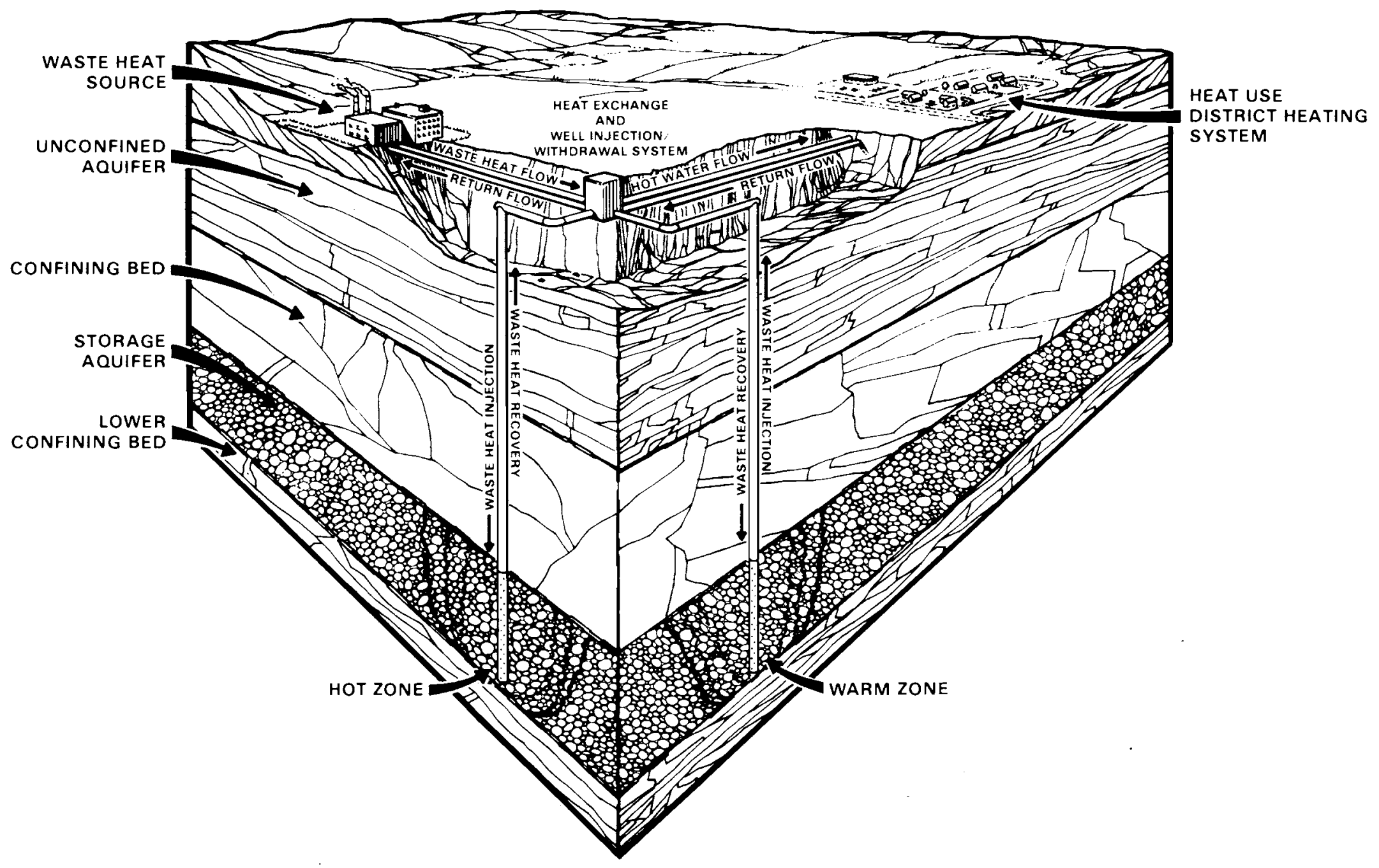

FIGURE 1. Schematic of Aquifer Thermal Energy Storage (after Reilly 1980) 
heated or chilled using an energy source, and reinjected back into the aquifer via the second well. After storage, the heated or chilled water is withdrawn from the second wel1, permitting recovery of the energy, and then reinjected back into the first well. This process is repeated many times throughout the life of the system. By using the storage aquifer as its own supplier one reduces the potential chemistry and particulate plugging problems. Another aspect of the doublet concept is that using a "closed system", the regulatory process may be easier.

During the last decade, the storage of thermal energy in aquifers has received considerable attention as a means to conserve and more efficiently use energy supplies. Since the geologic environment provides the storage container for the heated or chilled water, a thorough analys is of the hydrologic transport and thermal/chemical interactions in the geologic media used for storage is essential to a complete assessment of any ATES project. Because the analysis deals with a complex thermal/chemical problem, it inevitably involves models and simulation. Models provide the framework to incorporate all the processes that will be active in an ATES project, thereby permitting analysis and prediction of ATES behavior.

Models used for ATES studies may be divided into three general categories: (1) analytical, (2) numerical, and (3) geochemical. Analytical models are based on exact mathematical solutions to transport processes and are used where data and simplified assumptions permit. Numerical models for transport processes are based on equation approximations and matrix solution techniques. They are more general than analytical models, and are used for more complicated problems when supporting data permits. Geochemical models, on the other hand, are based on the solution to a set of chemical reaction and related equations. In general, geochemical models calculate the nature of chemical reactions, but not whether the reaction actually will occur or its rate of occurrence. Because of this lack of rate information, geochemical models are usually not incorporated directly into the analytical or numerical models. 
The purpose of this paper is to review the state-of-the-art modeling of aquifer thermal energy storage including (1) physical, chemical, and energy mechanisms, (2) mathematical treatment of transport mechanisms, (3) simulation techniques for permeable media, (4) techniques for analyzing geochemistry, (5) applications, and (6) site-specific considerations.

\section{LITERATURE REVIEW}

Aquifer storage is not a new idea; since aquifers have been used to store fresh water, petroleum products and liquid wastes. Kazmann (1971) was perhaps the first to suggest that aquifers might be used for ATES. However, the injection of heated or cooled fluid into aquifers has been practiced for over 45 years. Leggetts and Brashears (1938) describe the use of ground water for air conditioning on Long Island, New York, and its subsequent reinjection at temperatures that were increased by $10-15^{\circ} \mathrm{F}$. An early example of ATES is presented by Guyton (1946). In this example, cool water was injected during the spring into a glacial outwash aquifer near Louisville, Kentucky. The project was mainly concerned with artificial recharge, but the cool water was subsequently pumped out and used in the summer by Seagram and National distilleries to make alcohol (non-drinkable) for the war effort. As a result, during the summer when the city water became too warm to be used in the plants, an increased and ample supply of cold water was available from wells.

The injection of hot water as a means of enhanced petroleum recovery has also been performed for several years. A summary of information on $0 i 1$ recovery by steam and hot-water injection is provided by Ramey (1967) and Farouq Ali (1970).

Initial studies involving ATES were made by Rabbimov et al. (1971), Meyer and Todd (1973a), Meyer et a1. (1972), Me:er and Todd (1973b), and Hausz. (1974). These early works were mainly concerned with economical and institutional considerations. Werner and Kley (1975) reported results from an initial field experiment where $430 \mathrm{~m}^{3}$ of water at $45^{\circ} \mathrm{C}$ was injected into an aquifer over a 64-day period. Mathey (1977) also reported results from a field experiment where $494 \mathrm{~m}^{3}$ of hot water at $51^{\circ} \mathrm{C}$ was injected during a 
period of 223 hours. The water was partially recovered after a storage period of 4 months, with natural convection having the predominant effect. Another field experiment was reported by Molz et al. (1978) in which water was injected at $36^{\circ} \mathrm{C}$. The injected volume of water was kept in storage for a period of time and then recovered. Clogging was a serious problem in this experiment; however, enough information was gained to allow numerical simulation of the injection-storage-recovery cycle (Papadopulos and Larson 1978).

The preceding discussion represents only a partial literature review. The references presented in this section as well as additional references will be reviewed in more detail in subsequent sections. Our goal is to summarize theoretical and modeling studies and provide the reader with a realistic vantage point by summarizing field studies. Although the main emphasis of this report is on modeling procedures for ATES, discussions of current and former field studies and applications will provide the framework for assessing model needs.

Because ATES is a concept in which interest has only recently evolved, the literature is somewhat limited. To fill in gaps of the ATES literature, we have included information from the related fields of natural gas storage, geothermal reservoir engineering, and hot water and steam flooding of petroleum reservoirs. For current and recent studies, the reviews by Tsang et a1. (1980a) and Meyer et al. (1976), as well as the Proceedings of the Thermal Energy Storage in Aquifers Workshop and the quarterly ATES Newsletter have been very helpful. 


\section{CONCLUSIONS AND RECOMMENDATIONS}

It should be emphasized that aquifers are highly site-specific in their characteristics. While certain features may be generalized, the number of relevant parameters required to characterize a specific aquifer for ATES is large and unique (Hooper and Angus 1979). For this reason, many of the conclusions must be qualitative and the general modeling results presented in an illustrative manner.

To use and model an aquifer as a storage medium requires more detailed knowledge of the aquifer's characteristics than conventional uses of aquifers for water supply. This required information is not generally obtained for conventional applications, but it can be determined to the required degree of detail by currently available methods (Hooper and Angus 1979).

Models have been successfully used in ATES for both field studies and generic studies. Most of the basic physical processes active in ATES can be adequately represented in a model. These include fluid flow, heat conduction and convection, consolidation, solute transport, and two-phase flow. For field studies, models have been demonstrated as effective for preliminary feasibility modeling, conceptualization, and field data collection. A conclusive demonstration of predictive analysis is yet to be performed. Generic studies have been helpful in determining what factors and parameters most strongly affect recovery. For example, it has been demonstrated that the recovery factor increases with additional storage cycles.

It is evident that the solution of problems related to ATES usually requires an understanding of the coupled behavior of interacting physical and chemical processes. This understanding, in general, is best obtained by careful analysis of the processes in the field and laboratory. Once this is accomplished, the conceptual models of these processes can be translated into mathematical models that are suitable for predictive purposes. It seems that mathematical models, both analytical and numerical, are sufficiently powerful to solve many ATES applications. Unfortunately, the conceptual basis of some of these mathematical models appears to need 
reinforcement. This is especially true for incorporating chemical and biological reactions into models. Even if the mathematical models were known to be correct, the essential parameters describing field conditions are often unavailable.

As indicated in this review, there are many models available that consider a wide range of applications. Therefore, it appears that little additional model development is necessary unless and until available codes are applied to complete and representative data sets from typical ATES facilities. One question that has not been addressed adequately by modeling and field studies concerns economics. Limited economic studies have been conducted, but, in general, do not depend on detailed aquifer modeling results. A modeling approach to this problem is to combine an economic model with a reservoir model. Such an approach has been taken for geothermal development by Maddock et a1. (1979). Similar work for ATES should complement field demonstration projects that are completed to production.

Mathematical models will continue to play an important role in the design, operation, and environmental assessment of aquifer thermal energy storage. These models span the range from the very simple to the very complex. The major conclusions and recommendations concerning these models and their applications to ATES may be summarized as:

- Model application to field sites in conjunction with data collection activities is highly recommended to confirm the adequacy of the assumptions fundamental to currently available codes.

- There is little need for additional development of aquifer simulation models until the capabilities of the available models have been applied to and validated against the entire range of potential ATES applications.

- While geochemical and biological models are available, their thermodynamic and reaction rate data bases for application to the temperature range of ATES must be developed and documented.

- The physics of nonisothermal unsaturated flow should be investigated and available models validated against laboratory or field studies before the potential of unconfined aquifers for ATES can be estimated.

- The optimization of well field design (e.g., multiple wells) should be studied under constraints of gradient control, flow rates, energy recovery efficiency, and energy recovery quality. 
- A potentially useful management tool lies in the combination of reservoir and economic models to provide a design and operational scenario which is optimal in an economic sense.

- Fractured aquifers, in general, are not favorable for ATES, and development of models for this type of media is not warranted for the ATES program.

- ATES is mainly concerned with near-well performance and environmental effects away from the well; both of these problems can be addressed by present technology.

- Transport models are generally complex and difficult to use, requiring the user to be highly trained in order to avoid misuse.

- Costs associated with modeling may be divided into computer costs and mantime costs. In general, mantime expenses are much greater than computer expenses.

- ATES applications require a considerable amount of detailed field data.

- The adequacy of the field data will be the major factor controlling the credibility of any analysis and prediction. 


\section{PHYSICAL AND CHEMICAL PROCESSES}

An ATES project is affected by both natural and man-induced processes within an aquifer and wells. Physical-and energy-related aspects of an ATES project include physical, chemical and biological processes associated with ground-water flow and heat transport. Understanding what these processes are and the driving forces controlling them is essential to the operational efficiency of ATES. These processes are described in quantitative terms by the use of mathematical models.

Distributed processes that may be considered part of ATES are fluid flow, heat transport, solute transport, and deformation. Even though only four general processes are identified, the number of different models that can be conceived is very large. The reason for this is that many of the model variations are application dependent. In addition to applicationdependent variations, for convenience, equations describing the same process are often posed in terms of different dependent variables. Pressure is usually used as the dependent variable (also called unknown or state variable) in the ground-water flow equation for ATES, but hydraulic head is sometimes used. Table 1 provides a summary of the major processes, dependent variables, and application-dependent variations that are commonly encountered in ATES applications.

\section{FLUID FLOW}

Fluid movement is controlled by the transmissive properties of the medium and the potential gradient, with transient effects controlled in large part by the storage properties of the medium. For ATES there are several aspects of fluid flow that should be considered. These include natural or regional ground-water flow, flow due to withdrawal or injection, and flow due to changes in temperature. All of these are strongly dependent on the aquifer hydraulic properties, especially the aquifer permeability. This parameter determines the ability of an aquifer to transmit water under an applied potential gradient. It is important since it, in part, establishes the economical rate of water injection and withdrawal. 


\begin{tabular}{|c|c|c|}
\hline \multicolumn{3}{|c|}{$\begin{array}{l}\text { TABLE 1. Processes with Dependent Variables } \\
\text { and Application Variations }\end{array}$} \\
\hline Major Process & Dependent Variable & Application Dependent Variations \\
\hline Fluid Flow & $\begin{array}{l}\text { Fluid Pressure, } \\
\text { Hydraulic Head, } \\
\text { Hydraulic Potential } \\
\text { or Drawdown }\end{array}$ & $\begin{array}{l}\text { Porous Media } \\
\text { Doubly Porous Media } \\
\text { Discrete Fractured Media } \\
\text { Single-Phase Fluid } \\
\text { Two-Phase Fluid }\end{array}$ \\
\hline Heat Transport & $\begin{array}{l}\text { Temperature, } \\
\text { Enthalpy or } \\
\text { Internal Energy }\end{array}$ & $\begin{array}{l}\text { Same as for Flow plus } \\
\text { Convection } \\
\text { Conduction } \\
\text { Radiation }\end{array}$ \\
\hline Solute Transport & Concentration & $\begin{array}{l}\text { Same as for Flow plus } \\
\text { Convection } \\
\text { Dispersion } \\
\text { Chemical Source Sink Terms } \\
\text { Ion-Exchange } \\
\text { Adsorption \& Desorption } \\
\text { Precipitation \& Dissolution }\end{array}$ \\
\hline
\end{tabular}


Idea11y, the natural movement of ground water must be slow enough to a11 ow containment and retrieval of stored water, that is, so the injected volume of water does not drift away from the intended storage area. If drifting is likely to occur, it can be handled in two ways. First, for a we11 pair, the withdrawa 1 well can be appropriately located down gradient of the injection well such that heat recovery is optimized. And secondly, for either a single or a well pair system, gradient control wells can be placed and operated beyond the immediate influence of the heated region (Molz and Be11 1977).

The flow due to injection or withdrawal is a controllable flow rate dependent on the permeability of the porous medium and the pumping equipment used. The permeability can be affected by the chemistry of the injected water. If the injected water is not chemically compatible with the ambient ground water, reactions may occur causing precipitation of dissolved species, and dispersion or swelling of clay, all of which can clog the formation. It is imperative to the economic and hydraulic efficiency of ATES to maintain or, if possible, increase the permeability of an aquifer. This can be accomplished by periodic surging or possibly chemical treatments. Further discussion as to the chemical nature of clogging is discussed later. The optimal operation of ATES is site- and need-specific. The generally mentioned approach is injection followed by a "dormant" storage period followed by withdrawa1. Other options include injection followed by immediate withdrawal or possible injection and withdrawal conjunctively, if a multi-well system is used.

The flow due to the density differences between the hot and cold water has been termed buoyancy flow. This flow causes the hot water to rise and the cool water to sink. This action tilts the thermal front and ultimately leads to stratification, with the hot water above the cool water. These effects become more pronounced in thick and/or highly permeable aquifers having a low horizontal to vertical anisotropy, and when storage times are long or when the temperature difference between the stored and ambient fluids is large. Note that a trade-off exists between hydraulic efficiency and buoyancy flow with regard to an optimal permeability when the media is homogeneous. 
In addition to the transmissive characteristics of the aquifer, the storage properties must also be considered. Adequate storage volume must be demonstrated, which includes parameters such as effective porosity and aquifer thickness. The effective porosity of a formation is the percentage of accessible void space it contains.

Other hydraulic factors that affect an ATES project include aquifer depth, confinement and deformation properties. Aquifer depth is important, since it directly affects drilling and pumping costs. It also partially governs the maximum storage temperature and pressure. Injected fluid should be contained during storage to allow high energy recovery rates. Low permeability and thermal conductivity of overlying and underlying units are desirable to prevent fluid and thermal leakage.

In terms of deformation, there are two potential problems that should be controlled, land subsidence or uplift and hydraulic fracturing. There are two kinds of pressure to consider in underground strata (Katz and Coats, 1973): filid pressure in the pores of the rock and the overburden or lithostatic pressure exhibited by the solids. If the fluid pressure is reduced substantially, land subsidence may result; if it is increased, uplift may occur. If the fluid pressure is increased even further, so that it is $50-70 \%$ of the 1 ithostatic pressure, hydraulic fracturing may occur.

In addition to these aquifer properties that effect fluid flow, the fluid properties of density and viscosity also affect flow. Density, defined as mass of the fluid per unit volume, generally depends on pressure, temperature, and the chemistry of the water. Water density decreases with increasing temperature. Fluid viscosity or dynamic viscosity is a measure of the fluid's resistance to flow. It varies considerably with temperature, the decrease in viscosity with increasing temperature being almost exponential. Viscosity also exhibits a minor dependence upon water chemistry. These changes in density and viscosity cause the governing equations for nonisothermal flow to be nonlinear. 


\section{HEAT TRANSPORT}

When water is injected into an aquifer at a different temperature, it transfers thermal energy to the rock and water in the aquifer and to any surrounding confining units (Schaetzle et al. 1980). Thus, heat transport, and consequently, thermal energy storage occur in both the fluid and solid parts of an aquifer. Important aspects of ATES are conduction, thermal dispersion, convection and heat capacity.

Conduction-Dispersion

Conduction is defined as the direct transfer of heat through a substance by means of collisions with neighboring molecules due to molecular vibration. Heat flow by conduction is a result of a temperature difference and is described by Fourier's Law. Fourier's Law relates heat flow to a temperature gradient by a proportionality factor, which is thermal conductivity. A substance with a large thermal conductivity is a thermal conductor whereas one with a small value is a thermal insulator. The thermal conductivity of a liquid usually increases as the temperature is raised. Many rocks show a reduction of thermal conductivity with temperature increase (Clark 1966). These changes, however, are usually minor in most ATES applications.

The conductivities of rock and water are often combined into a thermal conductivity of fluid-saturated rock or medium thermal conductivity. Considerable work has been done on the medium thermal conductivity, and Somerton et a1. (1974) point out that it can be a function of temperature, porosity and water saturation.

In addition to the thermal conductivities of rock and water, in porous media, heat can be transferred as a result of velocity and temperature fluctuations in the pore space. Although at the microscopic level (see Appendix A) this is a form of convection, at the macroscopic level it appears as an enhanced conduction. Therefore, it is sometimes combined with the medium thermal conductivity to form a medium conduction-dispersion coefficient. A more complete discussion of thermal dispersion may be found in Bear (1972). It is similar in nature to the hydrodynamic dispersion associated with solute transport, which is discussed later. 
In terms of ATES, the effect of this term is to smear or spread temperature fronts. That is, for a large value of the medium conductiondispersion coefficient, hot water injected into an aquifer will tend to disperse far away from the well, reducing heat recovery efficiency when the well is pumped out.

\section{Convection}

In a general sense, convection is defined as the transferal of heat by the actual motion or flow of the hot fluid. For instance, the bulk movement of fluid through an aquifer causes heat transfer via convection. If the fluid is made to move by the action of a pump, the phenomenon is called forced convection, whereas advection has been used to describe convection associated with regional ground-water flow. If the motion of the fluid is caused by a difference in density that is a result of a temperature difference, the phenomenon is called natural or free convection. This has also been called buoyancy flow, and in ATES is most noticeable at the thermal front where hot fluid moves over cooler fluid due to density differences. If the storage aquifer is highly permeable (especially in the vertical) or if large storage times are planned, stratification may occur which can reduce the efficiency of the therral energy recovery.

\section{Heat Capacity}

Heat capacity is a term that indicates how well a material adsorbs heat for a given change in temperature. The heat capacity of a system per unit mass is called specific heat capacity or specific heat. It can be determined under conditions of either constant pressure or constant volume. Laboratory measurements of the heat capacity of solids and liquids, however, usually take place at constarit pressure. For liquid water below $100^{\circ} \mathrm{C}$, specific heat at constant pressure and constant volume are nearly equal.

Water has a high specific heat capacity, so it takes a considerable amount of heat to raise its temperature. Due to its high specific heat, water can store large amounts of heat. The specific heat capacity of the rock is approximately $25 \%$ that of water. However, for a sand having a $10 \%$ porosity, the rock occupies $90 \%$ of the porous medium. 


\section{SOLUTE TRANSPORT}

In general, solute transport should play a minor part in an ATES project. If the need does arise to understand solute transport, then two important factors should be discussed, hydrodynamic dispersion and source term properties.

\section{Dispersion}

Dispersion refers to the spreading and mixing caused in part by molecular diffusion and microscopic variation in velocities within individual pores (Anderson 1979). For many field problems, molecular diffusion (described by Fick's $1 \mathrm{aw}$ ) is small compared to mechanical mixing. The form of the dispersion tensor is complex and is discussed by Scheidegger (1961). A simplified representation that illustrates the basic components may be written as

$$
\overline{\bar{D}}=f\left(v, d_{1}, d_{2}\right)+f\left(D_{d}\right)
$$

where $f$ indicates "a function of" and $D_{d}$ is a molecular diffusion coefficient $\left(L^{2} / t\right)$. The first term to the right of the equal sign represents the mixing term and is composed of the real velocity (that is, Darcy velocity divided by effective porosity) and the longitudinal $\left(d_{1}\right)$ and transverse $\left(d_{2}\right)$ dispersivities (L). Dispersion requires at least six components since the velocity components can vary direction from point to point. In general, dispersion is determined by history matching and adjusting the dispersivities, $d_{1}$ and $d z$. Anderson (1979) discusses some of the difficulties in determining these parameters and presents tabulated values. These results, in part, are shown in Table 2. As noted, dispersion spreads the concentration distribution, and the larger the dispersivity, the larger the spreading. As may be seen from Table 2, there is a considerable range of values, with longitudinal (parallel to the direction of ground-water flow) dispersivity generally being larger than transverse (perpendicular to the direction of flow) dispersivity. These values are presented to help guide selection of dispersivity, but should not be strongly adhered to. 
TABLE 2. Regional Dispersivities Determined from Areal Model Studies (after Anderson 1979)

\begin{tabular}{|l|c|c|}
\hline \multirow{2}{*}{\multicolumn{1}{|c|}{ Material }} & \multicolumn{2}{|c|}{ Range $(\mathrm{m})^{+}$} \\
\cline { 2 - 3 } & $\begin{array}{c}\text { Longitudinal } \\
\text { dispersivity }\end{array}$ & $\begin{array}{c}\text { Transverse } \\
\text { dispersivity }\end{array}$ \\
\hline Alluvial sediments & $12-61$ & $4-30$ \\
Giacial deposits & 21 & 4 \\
Limestone & 61 & 18 \\
Fractured basalt & $30-91$ & $18-136$ \\
\hline
\end{tabular}

+ The implied precision in these numbers is mainly the result of metric conversion from original references. 
To illustrate the effects of dispersion, consider the concentration movement between parallel rows of injection wells and pumping wells in a confined homogeneous aquifer, where the wells are injecting at $c^{*}=1$. If we assume that the initial aquifer concentration is $\mathrm{C}=0$ and that steadystate flow exists between the wells, the problem can be approximated by a simple one-dimensional model (see Figure 2). The concentration profile without dispersion would appear as a sharp front that moves out at the average fluid velocity from the injection wells. With dispersion the front is smeared out.

The form of the dispersion term presented in Equation (1) is the one most commonly used in applications. However, the nature of the dispersion phenomena and its precise mathematical form are still sources of controversy. Two problems are commonly mentioned. Firstly, values determined by observations from laboratory and field data are scale dependent. Values of longitudinal dispersivity, $d_{1}$, vary from a few centimeters for laboratory experiments, through a few meters for tracer studies, to tens or hundreds of meters for regional pollution problems. The commonly accepted explanation for this is that the longitudinal dispersivity coefficient is a measure of the scale of heterogeneity that is not included in the analysis (the larger the area, the larger the dispersion). The second difficulty with the dispersion term as presented in Equation (1) is that it contradicts certain details of observations. In particular, since it depends primarily on the magnitude of the velocity field, it predicts dispersion of concentrations up gradient, that is, dissolved material movement opposite in direction to local ground-water flow. This effect is not observed in laboratory or field experiments. Figure 3 illustrates the typical discrepancy between observed and predicted results. In spite of ongoing debate concerning dispersion and its known limitations, insufficient agreement among the research community exists to offer a suitable alternative to describe the dispersion-mixing phenomena. For a further discussion on dispersion, see Fried (1975) or Bear (1972). 


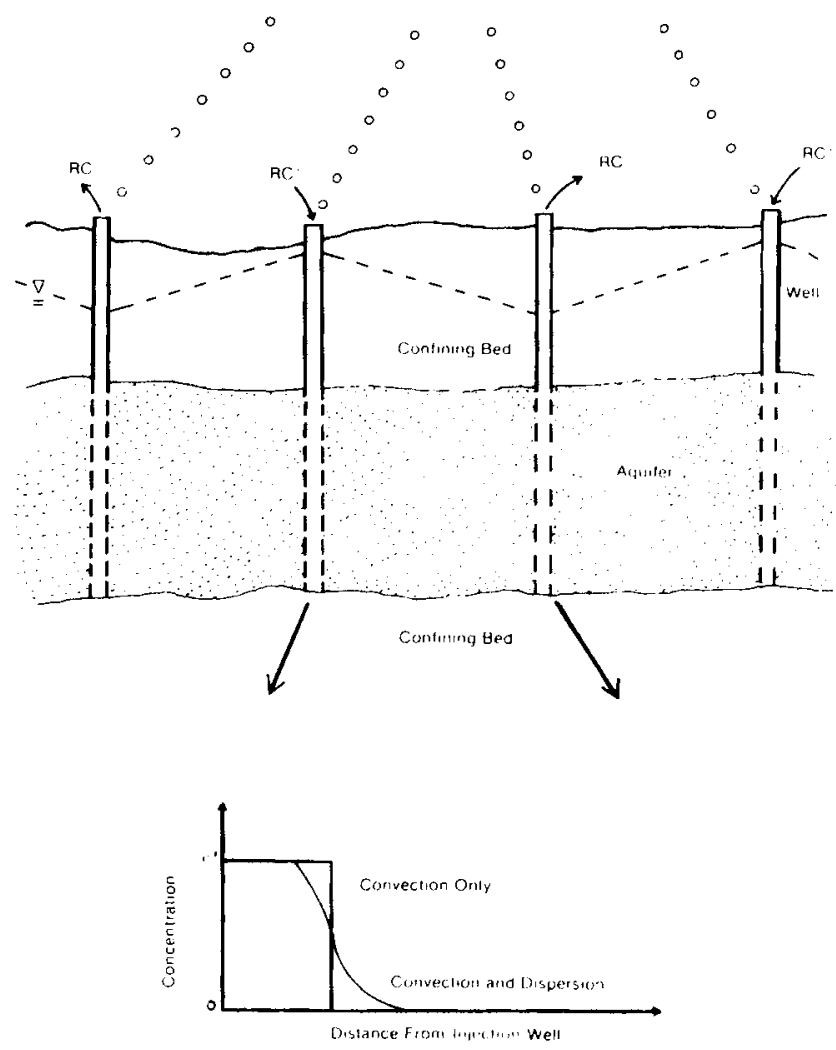

FIGURE 2. Idealized injection-recovery problem illustrating concentration front movement between injection and recovery wells. With convection only, concentration moves out as a sharp front. With dispersion, the front is smeared (from Mercer and Faust 1980). 


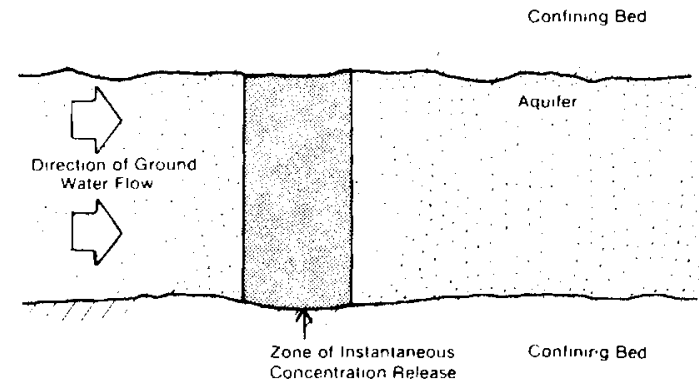

B

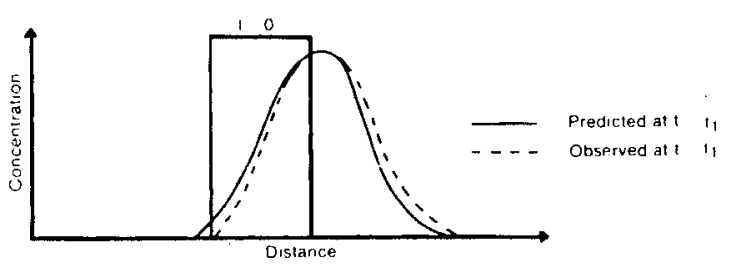

FIGURE 3. A) Physical situation of an instantaneous uniform concentration source in an aquifer. B) Typical discrepancy between observed and predicted concentration distributions for some later time $t_{1}$ (from Mercer and Faust 1980). 
Source Term Properties

Source terms can be incorporated that include the effects of the various chemical processes and reactions operating in the ground-water system. These include precipitation and solution, co-precipitation, oxidation and reduction, adsorption and desorption, ion exchange, complexation, ion filtration and gas generation. A common attempt to quantify sorption and precipitation downgradient of the source is through the use of distribution coefficients $\left(K_{d}\right)$. In general, a high $K_{d}$ indicates a strong tendency for sorption and therefore retards movement of material. Back and Cherry (1976) discuss this and other problems associated with incorporating geochemistry into transport models. A further discussion on adsorption may be found in Faust and Mercer (1980).

\section{GEOCHEMISTRY}

Only recently has work been done on the chemical reactions associated with ATES (Stottlemyre et al. 1979 and Stottlemyre et al. 1980). Therefore, most of the material in this section is taken from the fields of geothermal and petroleum reservoir engineering. In particular, we rely on references such as Ellis and Mahon (1977) and Patton (1974). Reactions that are considered are those that could change the permeability of the receiving formation or affect the equipment used to process and inject the fluid.

The problems that are considered include corrosion and mineral deposition. Modeling these problems proves to be quite difficult. As discussed in subsequent sections, one approach is to incorporate chemical reactions into a source term in the solute transport equation. Unfortunately, parameters characterizing this term are generally lacking. Another approach is to uncouple the chemistry from the flow through permeable media, and model just the chemical reactions. This approach is also discussed in a subsequent section.

of these two problems, mineral deposition is probably more important to ATES. A discussion on corrosion is included for completeness. This may be of importance in ATES projects that use solar energy, since high 
temperatures may cause the formation of steam. Under these conditions it will probably be necessary to use metal casing and the corrosion problems will be similar to those encountered in geothermal development. Thermal effects are also included, since temperature differences will be a major contribution to these chemical reactions.

\section{Corrosion Chemistry}

Corrosion chemistry associated with geothermal plants in New Zealand was reviewed by Marshall and Braithwaite (1973). These problems for geothermal fluids are probably more severe than corrosive problems in ATES, however, an outline of chemical aspects of geothermal fiuids should give insight into possible ATES problems.

The corrosion characteristics of fluids are generally examined by exposure tests, using the discharge of a typical well. Metals under appropriate stress conditions are exposed to the fluid. This exposure should account for the effects of aeration and fluid velocity. The corrosivity of the water is determined by such factors as temperature, $\mathrm{pH}$, types and amounts of salinity, velocity, and the presence of corrosive gases such as oxygen, carbon dioxide, or hydrogen sulfide (Subcasky 1973; Patton 1974). Corrosion products such as iron oxides and sulfides can plug the receiving formation when fluid is reinjected.

Mineral Deposition

When utilizing high-temperature water systems, consideration must be given to the possibility of mineral deposition, or more specifically, scaling. Changes in water temperature or pressure can cause the precipitation of certain solids. If this occurs in pipes or equipment, flow rates will be reduced and failure could occur. 


\section{Scaling}

Scaling can be described as the formation of a deposit or precipitate from a physical (e.g., temperature) or chemical (e.g., loss of carbon dioxide) change in a fluid (Subcasky 1978). It may also result from the mixing of two or more chemically incompatible fluids. Depending on kinetics and adhesion, the following cases can be distinguished (Subcasky 1978): (1) formation of a deposit on the walls of equipment, (2) sloughing of this deposit to give particles in solution, (3) formation of a suspended solid within the fluid, and (4) precipitation of a deposit on the sand grains of the formation.

\section{Calcium Carbonate}

According to Ellis and Mahon (1977), the changes in water chemistry which occur when steam boils from high-temperature water can be summarized as follows:

$$
\begin{aligned}
& \mathrm{CO}_{2}(\mathrm{aq})=\mathrm{CO}_{2} \text { (vap) } \\
& \mathrm{HCO}_{3}^{-}+\mathrm{H}^{+}=\mathrm{CO}_{2}(\mathrm{aq})+\mathrm{H}_{2} \mathrm{O} \\
& \mathrm{Ca}^{++}+2 \mathrm{HCO}_{3}^{-}=\mathrm{CaCO}_{3}+\mathrm{CO}_{2}(\mathrm{aq})+\mathrm{H}_{2} \mathrm{O} \\
& \mathrm{HCO}_{3}^{-}+\mathrm{H}_{3} \mathrm{BO}_{3}=\mathrm{H}_{2} \mathrm{BO}_{3}^{-}+\mathrm{CO}_{2}(\mathrm{aq})+\mathrm{H}_{2} \mathrm{O} \\
& \mathrm{HCO}_{3}^{-}+\mathrm{H}_{4} \mathrm{SiO}_{4}=\mathrm{H}_{3} \mathrm{SiO}_{4}^{-}+\mathrm{CO}_{2}(\mathrm{aq})+\mathrm{H}_{2} \mathrm{O}
\end{aligned}
$$

Water which is originally close to saturation with calcite rapidly becomes supersaturated following boiling, loss of carbon dioxide, and rising $\mathrm{pH}$. Most high-temperature geothermal waters contain concentrations of boric and silicic acids, and during the later stages of carbon dioxide loss there is a tendency for reactions of the type (5) and (6) to occur. 
Decreasing the pressure of a fluid without boiling may also cause the release of dissolved carbon dioxide. Again, the loss of carbon dioxide causes a rise in the $\mathrm{pH}$ of the fluid and a decrease in the solubility of calcium carbonate, i.e.,

$$
\mathrm{Ca}^{++}+2 \mathrm{HCO}_{3}^{-}=\mathrm{CaCO}_{3}+\mathrm{CO}_{2}(\mathrm{vap})+\mathrm{H}_{2} \mathrm{O}
$$

The solubility of most materials in water is affected by temperature (Subcasky 1978). For example, the solubility of calcium carbonate decreases with increasing temperature whereas the solubility of calcium sulfate (gypsum) increases with increasing temperature. Therefore, a solution saturated at one temperature may become supersaturated and form a precipitate at another temperature. The precipitate may form as an adherent deposit on the receiving formation or may form as a suspended solid which could plug the formation.

\section{Silica}

Silica deposition is relevant to the disposal of certain waters, since silica blockage of surface pipes and channelways during reinjection can cause great difficulties and expense. The reaction of solid silica with water may be written as:

$$
\mathrm{SiO}_{2}+2 \mathrm{H}_{2} \mathrm{O}(\mathrm{aq}) \underset{\overrightarrow{\mathrm{k}}_{+}}{\stackrel{\mathrm{k}}{\mathrm{k}}} \mathrm{H}_{4} \mathrm{SiO}_{4}(\mathrm{aq})
$$

where $k_{+}$is the rate constant for the forward dissolution reaction and $k$ is the rate constant for the reverse precipitation reaction. Both $k_{+}$and $k$ - are exponential functions of temperature and are related through the equilibrium constant. Additional information on these rates of reaction are given in Rimstidt and Barnes (1980).

In geothermal waters, the solubility of quartz may control the concentrations of silica. When discharged at atmospheric pressure, the fluid generally becomes supersaturated with respect to amorphous silica solubility. This may eventually be precipitated in various forms, including colloidal silica, gelatinous silica, and fibrous or opaline 
silica sinter (E1lis and Mahon 1977). The chemical processes leading to deposition are more complex than Equation (8) indicates.

\section{Mixing of Fluids}

While largely avoided when the doublet concept is employed, chemical problems may occur when fluids of differing compositions or concentrations are mixed. Problems of chemical incompatibility can arise when the injected fluid differs from the receiving fluid. Two examples from the petroleum literature are presented in Subcasky (1978). In the first, an injected water with a high sulfate concentration could form a plugging precipitate when mixed with a formation water with a high barium or calcium concentration:

$$
\mathrm{Ba}^{++}+\mathrm{SO}_{4}=\mathrm{BaSO}_{4}
$$

or

$$
\mathrm{Ca}+\mathrm{SO}_{4}=\mathrm{CaSO}_{4}
$$

In the second example, water with a high pH or alkalinity is mixed with another of high calcium or magnesium hydroxide:

$$
\mathrm{Ca}^{++}+\mathrm{HCO}_{3}^{-}+\mathrm{OH}^{-}=\mathrm{CaCO}_{3}+\mathrm{H}_{2} \mathrm{O}
$$

or

$$
\mathrm{Mg}^{++}+2 \mathrm{OH}^{--}=\mathrm{Mg}(\mathrm{OH})_{2}
$$

\section{WATER-ROCK INTERACTIONS}

Two topics are covered under water-rock interactions, dispersion of interstitial clay and particulate plugging. Modeling these processes in a rigorous fashion is impossible. Empirical formulas for permeability reduction may be used, but would be highly site-dependent and probably impossible to accurately predict a priori. Although these modeling difficulties exist, a discussion of the clay dispersion and plugging problems is presented. 
Dispersion of Interstitial Clay

Receiving formations usually contain clays and other minerals that may undergo physical and chemical changes when the electrolyte content of the surrounding fluid is changed (Mungan 1965 and Hewitt 1963). Therefore, the spacing between repeating layers of montmorillonite is altered when the degree of hydration is changed by the change in salt content (Subcasky 1978). When mica containing potassium ions (interplanar spacing 10 angstroms) comes into contact with a fluid that is low in potassium content, a cationic exchange or chemical reaction can occur with larger hydrated ions such as sodium or calcium. The interplanar spacing after the exchange is 15 angstroms (Reed 1977). Breaking and movement of clay particles may occur. Since the dispersing types of clays are charged species, changing the electric field around the particles by changing the electrolyte composition can cause the particles to disperse or coagulate (Subcasky 1978). Dispersed particles can migrate within the formation and plug narrow pores.

An example of this problem is presented in Brown and Silvey (1977). A decrease in specific capacity was observed during the injection of fresh water into a brackish-water sand aquifer. Hydraulic and chemical data indicated that the loss of specific capacity was due to dispersion of interstitial clay. Fortunately, the clay readily responded to chemical treatment for the purposes of decreasing or eliminating dispersion. The stabilization of interstitial clay was accomplished by injecting a pre-flush of calcium chloride solution in front of the fresh water.

According to Subcasky (1978), a rock formation may have an appreciable solubility in the injected water if the injected water is not in equilibrium with the formation. For example, water unsaturated with respect to calcium carbonate could dissolve the cementing carbonate present in some formations. Fine particles could be released which could plug the formation (Reed 1977). In silaceous formations this may also occur since nonequilibrium water at high $\mathrm{pH}$ and/or high temperature can dissolve considerable amounts of silica. Also, changes in $\mathrm{pH}$ and/or temperature may redeposit the dissolved material, causing formation plugging. 


\section{Particulate Plugging}

Particulate plugging may be the result of material in the injected fluid or from material formed or produced within the receiving formation. Possible sources of particulate matter in the injected fluid include (Subcasky 1978) silts, formation fines, corrosion products, scale deposits, bacteria, and precipitates from the mixing of chemically incompatible fluids. Water-rock or water-water interaction within the formation could produce or dislodge particles that could restrict flow through pores.

Particulate matter within the injected fluid can be highly variable. The rate and degree of permeability reduction depends on the particle properties, pore properties and flow rate (Donaldson and Baker 1977). Formation damage could be the result of a "filter cake" on the face of the injection well. A method for estimating the permeability of the filter cake is given by Collins (1961, p. 194).

An example of particulate plugging during an ATES project is given by Molz et al. (1978). During the first stage of this study, surface water was injected into a sand aquifer. Even though the water was filtered, particulate plugging occurred due to the inability to remove ciay and small silt-sized particles by filtration. Ultimately, the injection was terminated when increased injection pressure, induced by the clogging, led to failure of the upper confining layer along the well casing.

\section{BIOLOGICAL FACTORS}

Biological factors are also difficult to account for in a model by any means other than empirical formulas. Biological factors mainly consist of problems caused by microorganisms. For the purpose of ATES, microorganisms can be divided into three main groups (Patton 1974):

- Algae - contain chlorophyl1. They need sunlight to grow and can be a problem in surface ponds or open pits.

- Fungi - do not contain chlorophy11. Fungi do not usually constitute a major problem in injection operations. 
- Bacteria - have some properties in common with both fungi and algae. Bacteria comprise the broad class of microorganisms that are the most troublesome within an injection water.

The reason that bacteria can create so much trouble is that they can double their population in 20 minutes under many conditions. Bacteria can form slimes that plug a formation or filters. Colonies of bacteria on pipe walls can form oxygen concentration cells which accelerate corrosion and pitting (Subcasky 1978).

Sulfate reducers (Desulfovibria Desulfuricans) probably cause more serious problems than any other bacteria. They reduce sulfate ions in the water to sulfide ions, resulting in hydrogen sulfide as a by-product. Hydrogen sulfide is toxic and corrosive. Also, iron sulfide that is produced as a product of the corrosion reaction is an excellent plugging material.

Iron bacteria deposit iron hydroxide around them as they grow. The iron is obtained from soluble iron ions in the water. Iron bacteria can cause both corrosion and plugging. Although they do not directly participate in the corrosion reaction, corrosion can result either from the activity of sulfate reducers under the hydroxide sheath or by the creation of an oxygen concentration cell.

Slime forming bacteria are a general class of aerobic bacteria capable of producing dense masses of slime on solid surfaces. They cause plugging and contribute to corrosion in the same ways as iron bacteria by shielding part of the surface.

There are many other types of bacteria present in water injection systems. The sulfate reducers, iron bacteria and slime formers constitute the major microbiological problem. In general, chemicals are used to kill or inhibit the growth of these bacteria. For more detailed information on bacteria and fluid injection, see Patton (1974). 


\section{WELL FACTORS}

Well factors that should be considered when designing an ATES system include (1) well-bore heat loss and (2) production and monitoring well design and location. For general information on well design and construction, consult references such as Craft et al. (1962), Gibson and Singer (1971), or Campbe11 and Lehr (1973).

We11-Bore Heat Loss

We11-bore heat loss is the transferal of heat by conduction through the casing to the materials overlying the aquifer. It is strongly dependent on the casing material and the temperature of the injected/withdrawn liquid. For shallow aquifers (above 200 to $300 \mathrm{ft}$ ), this can be minimized by using polyvinyl chloride (PVC) casing instead of steel in that PVC is less conductive and less costly than steel; it is also corrosion resistant and of light weight (Schaetzle 1980).

\section{Well Design and Location}

The materials comprising an injection or withdrawal well, and its design, can affect the efficiency of an ATES project. As such, design decisions must be made for materials such as casing, liners, screens, fittings, pumps, grouts, and gravel packs, and towards any construction details such as screen settings. Much of this design criteria will depend upon the geohydrology of the storage formation (i.e., depth, hydraulic properties, and geology) and the money available to drill the wells. Pumping water from the aquifer, putting it through heat exchangers and injecting it again introduces considerable opportunity for entraining gases. Pumps, therefore, must be supplied with water at a pressure sufficient to prevent gas breakout and gas entrainment.

Another design criteria that should be considered is well penetration. When a well only partially screens a formation, inefficiency can occur upon withdrawal, that is, cooler water can be drawn in with the hot water (in the case where hot water is being stored). This is especially true when thermal stratification occurs and is referred to as water coning. Tsang (1980), however, shows the potential improvement in thermal recovery when 
full-penetration injection is followed by partial-penetration recovery.

We1l location depends in large part on the surface facilities as well as subsurface conditions describing the geohydrology. In many cases, analytical or numerical models may be used to give guidance in locating the wells. For example, a model may be used to simulate injection to determine the radial extent of a thermal front. This information can be used to site wells, either within or beyond the front. This same concept may be used to site gradient control wells, except that now the computed pressure gradient is used to help well siting. For a further discussion on well siting and well monitoring, see Warner and Lehr (1977). For legal and regulatory issues, see Hendrickson (1980). 


\section{MATHEMATICAL DESCRIPTION}

The variety and complexity of mathematical models (usually partial differential equations) used in ATES applications are numerous. This is possible because of significant advances in digital computers. This proliferation of mathematical models is often bewildering to the hydrologist who is trying to keep up with the research literature. Although the number of model types is large, as discussed, only a few basic processes are considered. This is somewhat ironic since the large number of "different" models is the result of various simplifying assumptions used to reduce a general set of equations to some solvable form. Fortunately, if one keeps in mind the fundamental processes being simulated, then different or simplified forms of equations are less confusing.

The development of a mathematical model begins with a conceptual understanding of the physical system. Once these concepts are formulated they can be translated into a mathematical framework resulting in equations that describe the process. A variety of analytical and numerical techniques can be applied to solve the equations, resulting in practical tools (often referred to as models) such as type curve or finite-difference and finiteelement computer programs.

In the last section, we discussed the processes and concepts associated with ATES and ground-water systems. In this section we look at the procedure for translating these concepts into mathematical terms. Rigorous development of specific equations used in ground-water applications may be found in textbooks (e.g., Bear 1972), and a simplified development is given in Appendix $A$. The emphasis here is on (1) a review of basic considerations and assumptions, (2) the procedure for obtaining the final equations, and (3) a discussion of the equations in physical terms. As part of this discussion, we also include boundary and initial conditions as they relate to the solution of the equations.

The derivations of equations used in ATES applications are based on the conservation principles dealing with mass, momentum, and energy. These principles require that the net quantity (mass, momentum, or energy) leaving 
or entering a specified volume of açuifer during a given time interval be equal to the change in the amount of that quantity stored in the volume. The derivation of the particular conservation equation involves representing the balance in terms of mathematical expressions. Once the balance equation is developed in mathematical terms, it is necessary to specify additional relationships among variables so that the equations can be solved. These include thermodynamic (e.g., the effect of fluid pressure on density) and constitutive (e.g., the effect of fluid pressure on porosity) relationships. The result of the derivation is usually a set of general partial differential equations in the three-dimensional cartesian coordinate system.

\section{GROUND-WATER FLOW}

\section{General Equation}

\section{Pressure Formulation}

An equation describing transient ground-water flow in three dimensions can be written (see Appendix A for derivation details) as

$$
\bar{\nabla} \cdot \frac{\bar{k} \rho}{\mu} \cdot(\bar{\nabla} p+\rho g \bar{\nabla} z)+\rho R=\frac{\partial(\phi \rho)}{\partial t}
$$

where

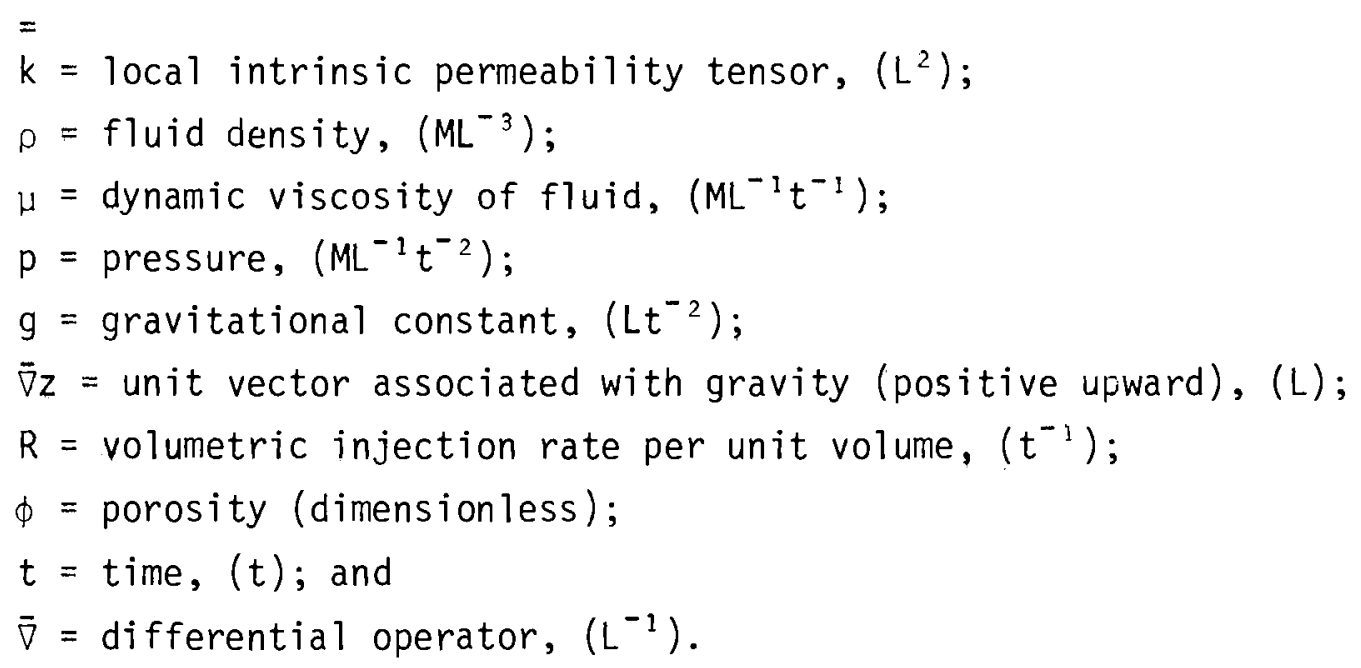


Since thermal effects in ATES applications may cause density changes, Equation (13) is written in terms of pressure. For general problems, al 1 of the equation parameters can vary from point to point within the aquifer (that is, they are functions of the spatial independent variables, $x, y$, and $z$ ). Equation (13) is derived by combining the mass conservation (water balance) and momentum conservation (Darcy's Law) equations, as shown in Figure 4. A better understanding of what Equation (13) expresses may be obtained by considering a small control volume of the aquifer. The first term in the equation represents the difference in the rate of water flowing into and out of the control volume. $R$ represents the rate of water gained or lost from some source or sink within the volume. The right-hand side represents the change in the amount of water stored in the control volume expressed as a rate.

Equation (13) is used in several reports on ATES. Sometimes the righthand side is expanded; this may be done as follows:

$$
\frac{\partial(\phi \rho)}{\partial t}=\phi \frac{\partial \rho}{\partial t}+\rho \frac{\partial \phi}{\partial t}
$$

In order to evaluate the density-time derivative, a constitutive assumption is needed relating density to temperature and pressure. Assuming that changes in density are sma11, density may be expanded about $\rho_{0}$ in a Taylor series truncated with first-order terms in temperature and pressure

$$
\rho=\rho_{0}+\left(\frac{\partial \rho}{\partial T}\right)_{0}\left(T-T_{0}\right)+\left(\frac{\partial \rho}{\partial p}\right)_{0}\left(p-p_{0}\right)
$$

where $\rho_{0}$ is the average density of the fluid at a reference temperature and pressure. The coefficients $\beta_{T}$ and $\beta_{p}$ may be defined as

$$
\beta_{T}=\frac{\left(\frac{\partial \rho}{\partial T}\right)_{0}}{\rho_{0}} \text { and } \beta_{p}=\frac{\left(\frac{\partial \rho}{\partial p}\right)_{0}}{\rho_{0}}
$$




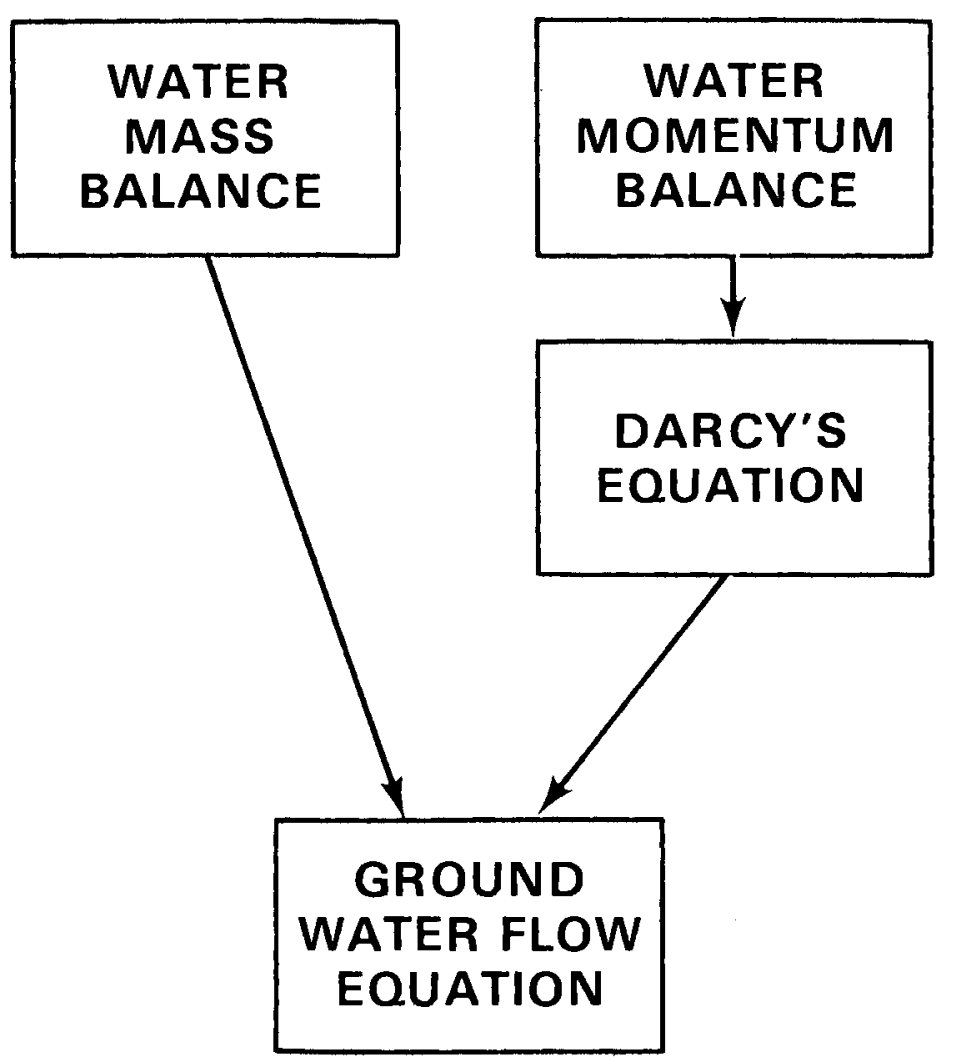

FIGURE 4. Major Components of the Ground-Water Flow Equation

(from Mercer and Faust 1980) 
where

$\beta_{T}=$ coefficient of thermal volume expansion for the fluid, $\left(T^{-1}\right)$;

$\beta_{p}=$ compressibility coefficient of the fluid, $\left(\mathrm{ML}^{-1} \mathrm{t}^{2}\right)$.

Porosity is assumed to be a linear function of pressure, giving an equation similar to Equation (15):

$$
\phi=\phi_{0}+\alpha\left(p-p_{0}\right)
$$

where $\phi_{0}$ is a reference porosity and $\alpha$ is the intergranular vertical compressibility coefficient, $\left(M^{-1} L t^{2}\right)$. Taking the time derivative of Equations (15) and (16), and substituting the result into Equation (14) gives

$$
\frac{\partial(\phi \rho)}{\partial t}=\left(\rho_{0} \beta_{p}+\rho \alpha\right) \frac{\partial p}{\partial t}+\phi \rho_{0} \beta_{T} \frac{\partial T}{\partial t} .
$$

Substituting Equation (17) into (13) yields an alternative form of the general equation

$$
\bar{\nabla} \cdot \frac{\bar{k} \rho}{\mu} \cdot(\bar{\nabla} p+\rho g \bar{\nabla} z)+\rho R=\left(\phi \rho_{c} \beta_{p}+\rho \alpha\right) \frac{\partial p}{\partial t}+\phi \rho_{0} \beta_{T} \frac{\partial T}{\partial t}
$$

Hydraulic Head Formulation

For general nonisothermal flow conditions in porous media, equations posed in terms of pressure are most appropriate. However, for applications in which temperature variations (and, hence, density variations) are small, hydraulic head may be used as the unknown dependent variable in the flow equation. Hydraulic head is related to pressure by (Hubbert 1940)

$$
h=z+\int_{p}^{p} \frac{1}{p g} d p
$$

where $z$ is the elevation above some reference datum and $p_{0}$ is some reference pressure. For hydraulic head to be uniquely defined, density must be a function of pressure only. In ATES problems, density is a function of both 
pressure and temperature, and an approximation of Equation (19) is required to pose Equation (18) in terms of head. The approximation is

$$
h \approx \frac{p}{\rho_{0} g}+z
$$

in which $\rho_{0}$ is the reference density of water - usually a typical value for water in the aquifer. Differentiating Equation (20),

$$
\bar{\nabla} p=\rho_{0} g \bar{\nabla} h-\rho_{0} g \bar{\nabla} z
$$

and

$$
\frac{\partial p}{\partial t}=\rho_{0} g \frac{\partial h}{\partial t}
$$

Substituting Equations (21) and (22) into (18) yields

$$
\begin{aligned}
\rho_{0} g\left\{\nabla \cdot \frac{\bar{k} \rho}{\mu} \cdot\right. & {\left.\left[\nabla h+\left(\frac{\rho}{\rho_{0}}-1\right) \nabla z\right]\right\}+\rho R=} \\
\rho_{0} g & {\left[\left(\phi \rho_{v} \beta_{p}+\rho \alpha\right) \frac{\partial h}{\partial t}+\phi \beta_{T} \frac{\partial T}{\partial t}\right] . }
\end{aligned}
$$

Expansion of density inside the parentheses on the left side of the equal sign as

$$
\rho \approx \rho_{0}+\rho_{0} \beta_{T}\left(T-T_{0}\right)
$$

and division by $\rho_{0}$ gives

$$
\bar{\nabla} \cdot \bar{K} \cdot\left[\bar{\nabla} h+\beta_{T}\left(T-T_{0}\right) \bar{\nabla} z\right]=S_{S} \frac{\partial h}{\partial t}+\phi \beta_{T} \frac{\partial T}{\partial t} \cdot
$$

The above equation uses the familiar ground-water parameters, hydraulic conductivity

$$
\begin{aligned}
& = \\
& \mathrm{k}=\frac{\rho g \mathrm{k}}{\mu}
\end{aligned}
$$


and specific storage

$$
S_{S}=g\left(\phi \rho_{0} \beta_{p}+\rho \alpha\right)
$$

Without the temperature terms, Equation (25) reduces to the conventional isothermal ground-water flow equation. The term $\beta_{T}\left(T-T_{0}\right) \vec{\nabla} z$ accounts for themal buoyancy effects. Although Equation (25) is in a convenient form, careful consideration to the approximations in Equations (20) and (24) should be given before applying it.

Variations of The General Equation

For many applications, a three-dimensional model may be neither necessary nor practical. One- and two-dimensional forms of the equation can be used by making certain assumptions concerning the symmetry or geometry of the system to be modeled. 0ther useful reduced forms of the three-dimensional flow equation include:

- two-dimensional, areal, cartesian coordinates

- two-dimensional, vertical, cartesian coordinates

- one-dimensional, cartesian coordinates

- two-dimensional, cylindrical coordinates

- one-dimensional, cylindrical coordinates

\section{Reduced Cartesian Equations}

Equation (13) can be integrated over the thickness of the aquifer (see, for example, Pinder and Gray 1977) to give

$$
\bar{\nabla}_{x y} \cdot \frac{b k_{\rho}}{\mu} \cdot\left(\bar{\nabla}_{x y} p+\rho g \bar{\nabla}_{x y}\langle z>)+\rho q^{\prime}+b \rho R=\frac{\partial(b \phi \rho)}{\partial t}\right.
$$

which is an areal ground-water flow equation where $\bar{\nabla}_{x y}$ now applies over the horizontal dimensions, $\langle z\rangle=\left(z_{\text {base }}+z_{\text {top }}\right) / 2$, and the additional source term $\rho q^{\prime}$ includes the gain or loss of water to leaky confining beds. There are several approximations for this leakage under either steady or transient flow conditions. As for Equation (13), the time derivative can be expanded in terms of pressure and temperature. The use of this equation is appropriate for problems in which the variation in equation parameters is small 
in the vertical dimension.

Reduced equations for the two-dimensional vertical slice and onedimensional horizontal cases can also be obtained by integration of Equation (13). These equations may apply to problems involving flow to a line of wells. For both equations, the limits of integration are over a unit thickness in the $y$-dimension and the aquifer thickness in the $z$-dimension. The $x-z$ equation is

$$
\bar{\nabla}_{x z} \cdot \frac{\bar{k}_{\rho}}{\mu} \cdot\left(\bar{\nabla}_{x z} p+\rho g \bar{\nabla}_{x z} z\right)+\rho R=\frac{\partial(\phi \rho)}{\partial t}
$$

and the $x$ equation is

$$
\frac{\partial}{\partial x}\left[b \frac{k \rho}{\mu}\left(\frac{\partial p}{\partial x}+\rho g \frac{\partial\langle z>}{\partial x}\right)\right]+\rho q^{\prime}+b \rho R=\frac{\partial(b \phi \rho)}{\partial t},
$$

where all terms have been defined previously.

\section{Radial Equation}

For applications involving radial flow to a vell, Equation (13) can be transformed to cylindrical coordinates. If it is also assumed that radial symmetry exists, then a simpler two-dimensional form of the equation can be used. The two-dimensional form of this equation is

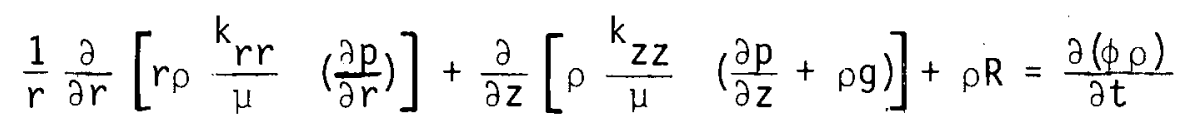

Again, the time derivative can be expanded in terms of pressure and temperature as in Equation (18).

The radial, one-dimensional equation is the basis for many of the solutions applied to aquifer testing. If it is assumed that vertical changes in ground-water flow parameters are sma11, Equation (29) can be integrated over the thickness of the aquifer, yielding

$$
\frac{1}{r} \frac{\partial}{\partial r}\left[\operatorname{br} \rho \frac{k_{r r}}{\mu}\left(\frac{\partial p}{\partial r}\right)\right]+\rho q^{\prime}+\rho b R=\frac{\partial(\phi \rho b)}{\partial t}
$$

in which all terms have already been described. 


\section{Isothermal Equations}

Although nonisothermal conditions will exist in an operating ATES system, isothermal conditions will normally prevail before injection begins. Consequently, a great deal of hydrologic analysis can be done using available data in conjunction with simple ground-water models. Some of the useful equations are listed below.

Three-dimensional cartesian:

$$
\bar{\nabla} \cdot \bar{k} \cdot \bar{\nabla} h=S_{S} \frac{\partial h}{\partial t}
$$

which is the diffusion equation.

Two-dimensional cartesian (areal):

$$
\bar{\nabla}_{x y} \cdot \bar{T} \cdot \bar{\nabla}_{x y} h+q^{\prime}+b R=s_{s} \frac{\partial h}{\partial t}
$$

in which $\overline{\bar{T}}=\vec{b} \bar{K}$ is called the transmissivity and $S=S_{S} b$ is the storage coefficient.

Two-dimensional, vertical slice, cartesian:

$$
\bar{\nabla}_{x z} \cdot \bar{k} \cdot \bar{\nabla}_{x z} h=s_{s} \frac{\partial h}{\partial t}
$$

Two-dimensional, cylindrical:

$$
\frac{1}{r} \frac{\partial}{\partial r}\left[r K_{r r}\left(\frac{\partial h}{\partial r}\right)\right]+\frac{\partial}{\partial z}\left[K_{z z} \frac{\partial h}{\partial z}\right]+R=S_{S} \frac{\partial h}{\partial t}
$$

One-dimensional, radial:

$$
\frac{1}{r} \frac{\partial}{\partial r}\left[r T\left(\frac{\partial h}{\partial r}\right)\right]+q^{\prime}+b R=S \frac{\partial h}{\partial t}
$$

Of the above equations, (32), (34), and (35) will probably be the most useful. The areal flow equation can be used to evaluate the existing regional flow field. Equation (35) has many analytical solutions for 
various boundary and leakage conditions. These solutions can be used to determine hydrologic parameters from aquifer tests. Models based on Equations (35) and (33) can be used to evaluate practical pumping and injection rates for alternative well-spacing designs.

\section{HEAT TRANSPORT}

\section{General Equation}

The ground-water flow equation describes the rate of propagation of a pressure or head change in an aquifer. In order to describe the movement of heat, the heat transport equation and the ground-water flow equation must be solved simultaneously.

The equation describing transport of heat energy in saturated porous media was developed in Appendix A. It was obtained by taking a heat energy balance as shown in Figure 5 and may be written as

$$
-\bar{\nabla} \cdot\left(\rho \bar{q} c_{p} T\right)+\bar{\nabla} \cdot\left(K_{m}^{\star} \bar{\nabla} T\right)+\rho R c_{p} T^{\prime}=\frac{\partial}{\partial t}\left[\phi \rho c_{p}+(1-\phi) \rho_{r} c_{p r}\right] T
$$

where the new terms are

$\bar{q}=$ flux, volumetric flow rate per unit area, $\left(\mathrm{Lt}^{-1}\right)$;

$c_{p}=$ heat capacity at constant pressure, per unit mass, $\left(L^{2} t^{-2} T^{-1}\right)$;

$T=$ temperature, $(T)$;

$\bar{K}_{m}^{\star}=$ medium conduction/dispersion tensor $\left(M L t^{-3} T^{-1}\right)$; and

where the subscript $r$ refers to rock and the superscript ' refers to the source/sink term. Note that the flux $\bar{q}$ is determined from Darcy's Law and a solution of the ground-water flow equation. Equation (36) is rigorously correct if $c_{p}$ is constant; otherwise, it is a valid approximation for the temperature ranges qenerally encountered in ATES $\left(0-150^{\circ} \mathrm{C}\right)$.

Equation (36) contains a convection term, a conduction/disperion term, a source term and an accumulation terin. It is similar to the ground-water flow equation, except with an additional term, and is called a convectiondiffusion equation. The medium thermal conduction/dispersion tensor in Equation (36) includes the effects of thermal dispersion, which is analogous to solute dispersion, discussed in the next section, and heat conduction 


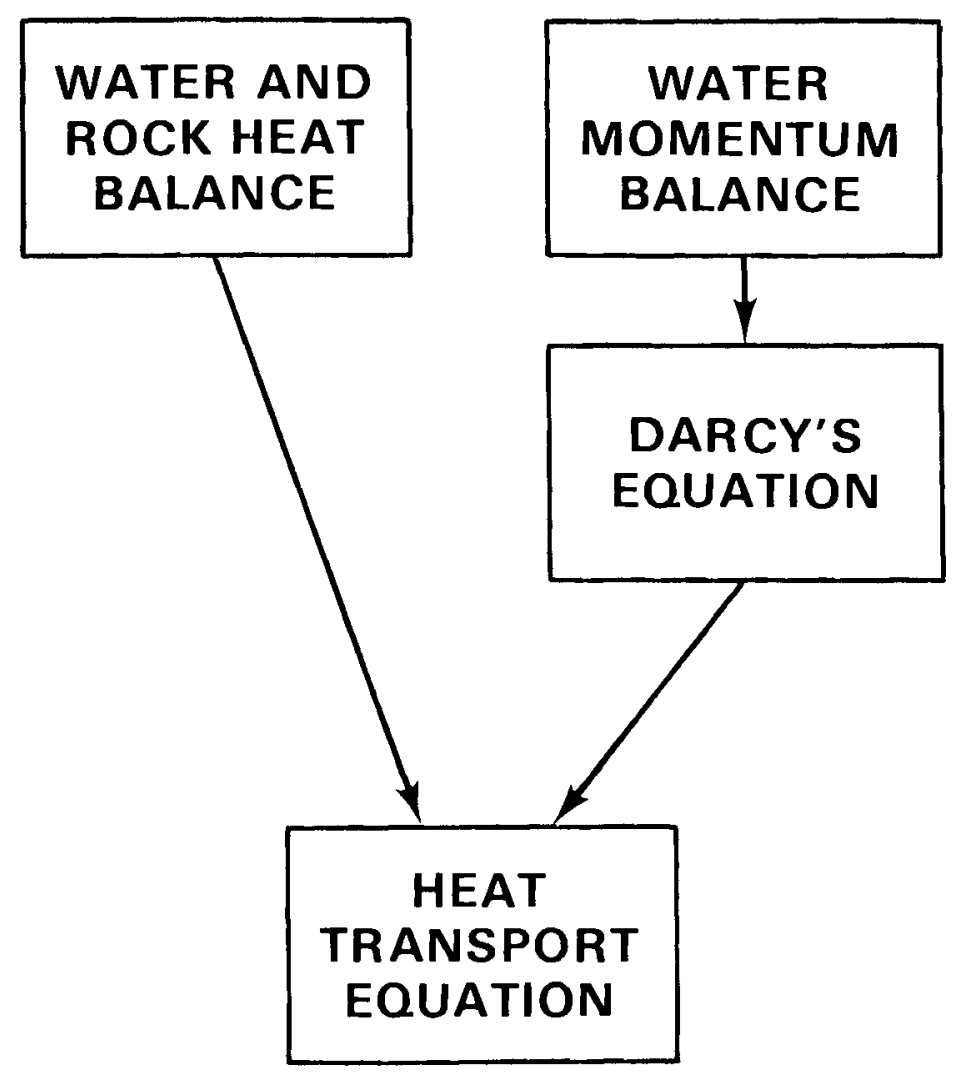

FIGURE 5. Major Components of the Heat Transport Equation

(from Mercer and Faust 1980) 
which is analogous to molecular diffusion. Unlike the solute dispersion term, it includes, and can be dominated by, thermal conduction in the rock. The conductive flow of heat is described by Fourier's Law, which has the same form as Darcy's Law. The derivation of Equation (36) is based on the assumption of thermal equilibrium between water and rock. This is generally reasonable since the movement of water in a porous medium is often slow, while the surface area of the water-rock contact is large.

Thermal dispersion is not a we11-understood phenomena. In heat transport, heat conduction is often as important as thermal dispersion. Because of this, it is very difficult to determine thermal dispersion properties from experimental or field data. Two forms of the dispersion tensor are used in the literature - one is an extension of the solute dispersion expression, the other is a lumped coefficient. In view of the lack of thermal dispersion data and the difficulty in determining it from the field, the lumped coefficient is often the more appropriate as well as more practical formulation. In the following paragraphs a brief review of the more general formulation of the thermal dispersion tensor is given.

The total thermal dispersion tensor is composed of two parts, dispersion in the liquid, and conduction in both solid and liquid:

$$
\bar{K}_{m}^{*}=\bar{K}_{L}+\bar{K}_{C}
$$

where

$$
\begin{aligned}
& {\overrightarrow{K_{m}}}^{\star}=\text { the total thermal dispersion tensor }\left(\mathrm{MLt}^{-3} \mathrm{~T}^{-1}\right) ; \\
& \overline{K_{L}}=\text { the thermal dispersion tensor }\left(\mathrm{MLt}^{-3} \mathrm{~T}^{-1}\right) ; \\
& \overline{K_{C}}=\text { the molecular heat conduction tensor of both water and } \\
& \quad \text { rock }\left(\mathrm{MLt}^{-3} \mathrm{~T}^{-1}\right) .
\end{aligned}
$$


A functional relationship is needed so that Equation (37) can be evaluated. As previously noted the total thermal dispersion tensor is analogous to the dispersion tensor used in solute transport work and is subject to the same analysis. There are several functional relationships for the equivalent of Equation (37) in solute transport literature given by Bear et al. (1968), List and Brooks (1967), Poreh (1965), and Scheidegger (1961). The development presented follows a development for the solute dispersion tensor given by Redde 11 and Sunada (1970).

The heat conduction mechanism is not exactly analogous to molecular diffusion in solute transport. Heat can be conducted through the rock as. well as through the water, whereas molecular diffusion occurs only in the water. Formulas for determining heat conduction coefficients based on the contribution from both rock and water are available (see Bear 1972). For field applications such complexity is rarely justified by available data. Consequently, a medium thermal conductivity coefficient - generally not a tensor - is estimated from data measured on similar rocks.

The second part of the total thermal dispersion tensor is the thermal dispersion tensor. Using summation notation, Scheidegger (1961) evaluates the dispersion tensor, $\overline{\bar{K}}_{L}$, as follows

$$
k_{i j}=\varepsilon_{i j k \ell} \frac{v_{k} v_{\ell}}{v}
$$

where

$$
\begin{aligned}
\varepsilon_{i j k \ell}= & \text { the dispersivity of the medium, a fourth rank tensor, }(L) ; \\
v_{k} v_{\ell}= & \text { components of velocity in the } k \text { and } \ell \text { directions, } \\
& \text { respectively, }\left(L t^{-1}\right) ; \\
v= & \text { magnitude of the velocity, }\left(L t^{-1}\right) .
\end{aligned}
$$


Scheidegger further demonstrates that for an isotropic media, the dispersivity tensor reduces to the following nonzero components:

$$
\begin{aligned}
& \varepsilon_{\text {rrr }}=\varepsilon_{1}, \\
& \varepsilon_{\text {rrs }}=\varepsilon_{2}, \\
& \varepsilon_{r s r s}=\frac{1}{2}\left(\varepsilon_{1}-\varepsilon_{2}\right), \\
& \varepsilon_{r s s r}=\frac{1}{2}\left(\varepsilon_{1}-\varepsilon_{2}\right) .
\end{aligned}
$$

The longitudinal and transverse thermal dispersion coefficients are related to the dispersivities by

$$
K_{\ell}=\varepsilon_{1} v
$$

and

$$
K_{t}=\varepsilon_{2} v .
$$

Expanding Equation (37), making these substitutions, and assuming isotropic heat conduction, the following functional relationship for the nine components of the total thermal dispersion tensor are obtained:

$$
\begin{aligned}
& k_{x x}^{*}=k_{l} \frac{v_{x} v_{x}}{v^{2}}+k_{t} \frac{v_{y} v_{y}}{v^{2}}+k_{t} \frac{v_{z} v_{z}}{v^{2}}+k_{c}, \\
& k_{y y}^{*}=k_{t} \frac{v_{x} v_{x}}{v^{2}}+k_{\ell} \frac{v_{y} v_{y}}{v^{2}}+k_{t} \frac{v_{z} v_{z}}{v^{2}}+k_{c}, \\
& k_{z z}^{*}=k_{t} \frac{v_{x} v_{x}}{v^{2}}+k_{t} \frac{v_{y} v_{y}}{v^{2}}+k_{l} \frac{v_{z} v_{z}}{v^{2}}+k_{c}, \\
& k_{x y}^{*}=k_{y x}^{*}=\left(k_{l}-k_{t}\right) \frac{v_{x} v_{y}}{v^{2}},
\end{aligned}
$$




$$
\begin{aligned}
& k_{x z}^{\star}=k_{z x}^{*}=\left(k_{l}-k_{t}\right) \frac{v_{x} v_{z}}{v^{2}}, \\
& k_{y z}^{\star}=k_{z y}^{\star}=\left(k_{l}-k_{t}\right) \frac{v_{y} v_{z}}{v^{2}} .
\end{aligned}
$$

\section{Variations of The General Equation}

As with the ground-water flow equation, the most general heat transport equation is not always the most appropriate to use. Two of the more useful equation subsets include the two-dimensional areal equation and the cylindrical $(r-z)$ equation. These are listed below (note in the listed equations, the total thermal dispersion tensor has been reduced to a single coefficient).

\section{Reduced Cartesian Equations}

Just as the ground-water flow equation was integrated over the thickness of an aquifer, the heat transport equation may also be partially integrated over the vertical to give

$$
\begin{gathered}
-\bar{\nabla}_{x y} \cdot\left(b \rho \bar{q} c_{p} T\right)+\bar{\nabla}_{x y} \cdot\left(b K_{m} \bar{\nabla}_{x y}{ }^{\top}\right)+b \rho R c_{p} T^{\prime}+q^{\prime} \rho c_{p} T^{\prime \prime}+Q= \\
\frac{\partial}{\partial t}\left[b \phi \rho c_{p}+b(1-\phi) \rho_{r} c_{p r}\right] T .
\end{gathered}
$$

In this equation two heat source terms arise from consideration of heat gained or lost to confining beds. The first ( $\left.q^{\prime} p c_{p} T^{\prime \prime}\right)$ represents the convection heat flux carried by water entering the aquifer at temperature $T^{\prime \prime}$. The second term $Q$ is the conductive heat loss or gain to confining beds. As with the ground-water flow equation, several alternative approaches are available for treating (or approximating) the confining bed source terms.

The areal heat transport equation can be used to examine flow in confined aquifers with small vertical variation in equation parameters. In general, this will not be the case unless injection and production wells fully penetrate the aquifer. 
Equations for a vertical slice $(x-z)$ and the horizontal ( $x$ ) may be obtained trivially by dropping the y terms from Equations (36) and (39), respectively.

\section{Radial Equation}

Assuming radial symmetry, the heat transport equation may be transformed to give

$$
\begin{aligned}
& \frac{1}{r} \frac{\partial}{\partial r}\left(r \rho c_{p} T q_{r}\right)+\frac{\partial}{\partial z}\left(\rho c_{p} T q_{z}\right)+\frac{1}{r} \frac{\partial}{\partial r}\left(r K_{m} \frac{\partial T}{\partial r}\right)+\frac{\partial}{\partial z}\left(K_{m} \frac{\partial T}{\partial z}\right)+\rho R c_{p} T^{\prime}= \\
& \frac{\partial}{\partial T}\left[\phi \rho c_{p}+(1-\phi) \rho_{r} c_{p r}\right] T .
\end{aligned}
$$

Integrating over the vertical dimension yields

$$
\begin{aligned}
& \frac{1}{r} \frac{\partial}{\partial r}\left(b r \rho c_{p} T q_{r}\right)+\frac{1}{r} \frac{\partial}{\partial r}\left(b r k_{m} \frac{\partial T}{\partial r}\right)+b \rho c_{p} R T^{\prime}+q^{\prime} \rho c_{p} T^{\prime \prime}+Q= \\
& \frac{\partial}{\partial t}\left[b \phi \rho c_{p}+b(1-\phi) \rho_{r} c_{p r}\right] T .
\end{aligned}
$$

Equation (40) can be used to evaluate the effects of thermal bouyancy in the vicinity of an injection well. The effects of thermal dispersion can be evaluated using either Equation (40) or (41). For idealized conditions Equation (41) has an analytical solution that can be used for calculations of a preliminary or very approximate nature.

\section{SOLUTE TRANSPORT}

In general, a complete physical-chemical description of ATES would include the movement of the fluid, heat, and all species of material dissolved in the fluid, and chemical reactions among the various species. The difficulties encountered in solving the set of equations descriping this interaction (for real problems) have forced hydrologists to consider simplified subsets of the general problem. In order to describe the transport of heat and dissolved chemical species in ground water, the two transport equations and the ground-water flow equation must be solved simultaneously. 
The transport equation for a solute is similar in form to the heat transport equation and is obtained by taking a mass balance of the material as shown in Figure 6. It may be written as (see, for example, Reddell and Sunada 1970)

$$
\bar{\nabla} \cdot \rho \phi \bar{D} \cdot \bar{\nabla} C-\bar{\nabla} \cdot \rho \vec{q} C+\rho R C^{\prime}=\frac{\partial(\rho \phi C)}{\partial t}
$$

where $C$ is the material concentration $\left(M / L^{3}\right), C^{\prime}$ is the concentration of the source/sink term $\left(M / L^{3}\right)$ and $\overline{\bar{D}}$ is the dispersion tensor $\left(L^{2} / t\right)$. The solute transport equation (also known as the convection-diffusion equation) contains (from left to right) a dispersion term, a convection term, a source term (which can include chemical reactions) and a rate change in concentration term.

Although Equation (42) mathematically describes solute transport, determining the parameters used in this equation usually proves to be quite difficult. These parameters include: (1) velocity, (2) dispersion properties, and (3) source term properties. Velocities are generally determined using Darcy's equation and solving the ground-water flow equation. Therefore, porosity, intrinsic permeability, as well as the pressure distribution, must be known.

For ATES applications, solute transport is not a major modeling consideration. For situations in which chemical problems exist (such as clay dispersion or mineral precipitation) the transport models are likely to be inadequate. The coupling of chemical reaction terms with the transport equation is still very much an area of current research. Because the water to be injected should have relatively high quality or be similar to the water withdrawn, conservative transport (no reactions) should not be of much concern either.

One of the models discussed later has the capability of simulating fluid flow, heat transport and solute transport simultaneously. However, it is more likely that geochemical models not considering solute transport will be more applicable to ATES chemical related problems. 


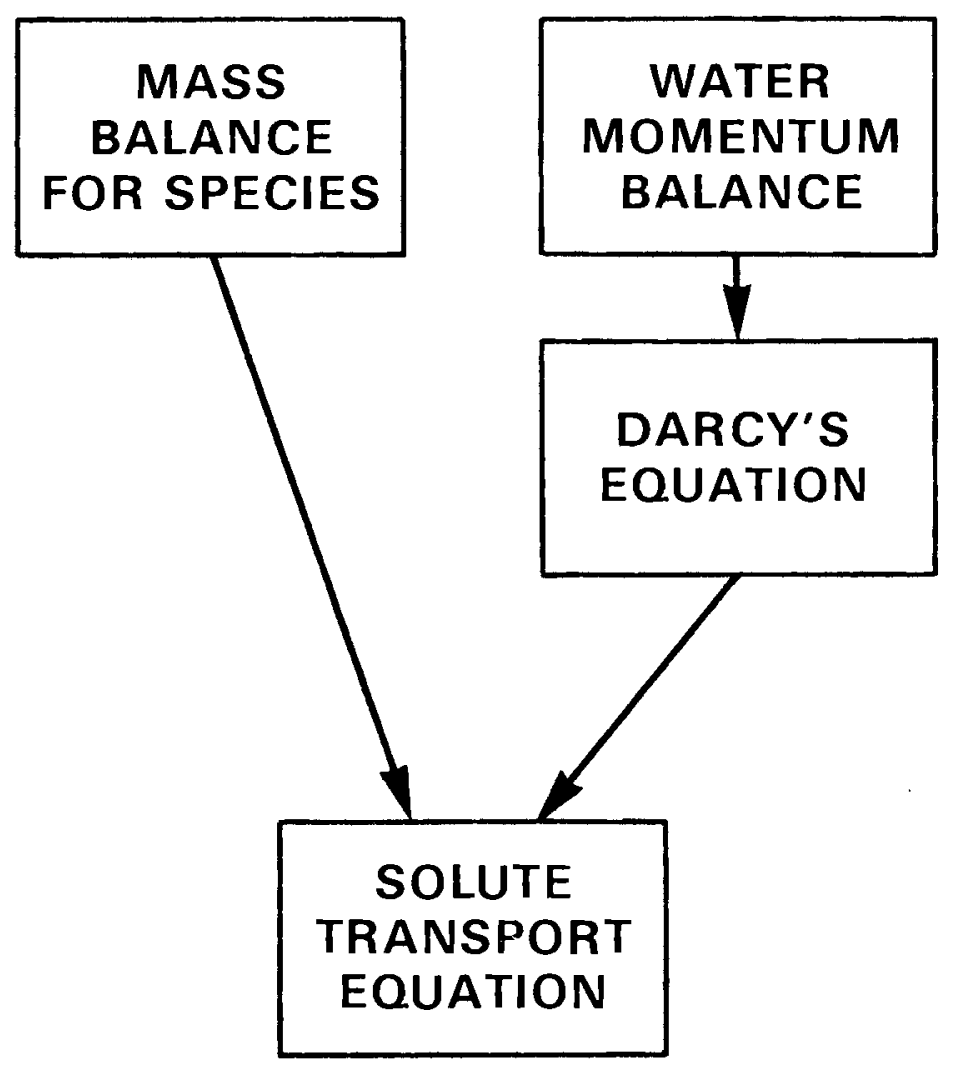

FIGURE 6. Components of the Solute

Transport Equation

(from Mercer and Faust 1980) 
BOUNDARY AND INITIAL CONDITIONS

In order to obtain a unique solution of a partial differential equation corresponding to a given physical process, additional information about the physical state of the process is required. This information is described by boundary and initial conditions. For steady-state problems only boundary conditions are required, whereas for transient problems both boundary and initial conditions are required. Mathematically, the boundary conditions include the geometry of the boundary and the values of the dependent variable or its derivative normal to the boundary. In physical terms, for ground-water applications the boundary conditions are generally of three types: (1) specified value, (2) specified flux or (3) valuedependent flux, where the value is potential, concentration or temperature, depending on the equation. The initial conditions are simply the values of the dependent variable specified everywhere inside the boundary at time equals zero.

For ATES applications the types of boundary conditions that may arise are summarized in Table 3. The specification of boundary conditions is of primary importance for (1) injection or production wells, (2) the external periphery of the area to be simulated, and (3) the contacts between the aquifer and its overlying and underlying confining strata.

Since the porous media stops at the well face, the aquifer not only has a boundary around its perimeter, but the outline of each well is also considered a boundary to the aquifer. The flow boundary conditions at wells are treated as constant or variable-specified flux, or constant head, depending on which best describes the actual physical conditions. If the well is discharging or recharging at a given concentration or temperature, then these must also be specified, when the transport equations are being considered. When using numerical modeis, it is not always evident that boundary conditions are specified at the wells. This is because the well boundary condition is usually lumped into a well source term. For analytical solutions involving a well, the boundary condition of the well is always stated explicitly. 
TABLE 3. Boundary Conditions for Ground-Water Flow, Heat Transport, and Solute Transport

\begin{tabular}{|c|c|c|c|c|}
\hline $\begin{array}{c}\text { Boundary Condition } \\
\text { Type }\end{array}$ & $\begin{array}{l}\text { Ground-water } \\
\text { Flow }\end{array}$ & $\begin{array}{l}\text { Heat } \\
\text { Transport }\end{array}$ & $\begin{array}{l}\text { Solute } \\
\text { Transport }\end{array}$ & Description \\
\hline $\begin{array}{l}\text { Specified Value } \\
\text { (Dirichlet) }\end{array}$ & $p=p(x, y, z, t)$ & $T=T(x, y, z, t)$ & $C=C(x, y, z, t)$ & $\begin{array}{l}\text { Values of pressure, tempera- } \\
\text { ture or concentration are } \\
\text { specified along the boundary. } \\
\text { Examples: temperature at the } \\
\text { well face, pressure at bound- } \\
\text { ary connected to large surface } \\
\text { water body. }\end{array}$ \\
\hline $\begin{array}{l}\text { Specified Flux } \\
\text { (Neumann) }\end{array}$ & $\begin{aligned} q_{n}= & -\frac{k \rho}{\mu}\left(\frac{\partial p}{\partial n}\right. \\
& \left.+\rho g \frac{\partial z}{\partial n}\right)\end{aligned}$ & $Q_{n}=-K_{m} \frac{\partial T}{\partial n}$ & $Q c_{n}=-D_{n} \frac{\partial C}{\partial n}$ & $\begin{array}{l}\text { Flux rate of water, heat, or } \\
\text { concentration is specified } \\
\text { along the boundary and equated } \\
\text { to the normal derivative. } \\
\text { Examples: } \\
q_{n}=0 \text { on an impermeable } \\
a_{n}=0 \text { on an insulated } \\
\text { boundary }\end{array}$ \\
\hline $\begin{array}{l}\text { Value-Dependent } \\
\text { Flux } \\
\text { (Mixed) }\end{array}$ & $q_{n}=q_{n}(p)$ & $Q_{n}=Q_{n}(T)$ & $Q c_{n}=Q c_{n}(C)$ & $\begin{array}{l}\text { The flux rate is related to } \\
\text { both the normal derivative and } \\
\text { the value. In ground-water } \\
\text { flow, this is a leaky or spring } \\
\text { type boundary condition. For } \\
\text { heat transport, it represents } \\
\text { heat loss to a boundary, such } \\
\text { as a confining bed. }\end{array}$ \\
\hline
\end{tabular}


For ground-water flow all three of the alternative boundary conditions are encountered in applications. Impermeable or nearly impermeable boundaries are formed by underlying or overlying beds of rock, by contiguous rock masses (as along a fault or along the wall of a buried rock valley), or by dikes or similar structures (Jacob 1950). Permeable boundaries are formed by the bottom of rivers, canals, lakes, and other bodies of surface water. These permeable boundaries may be treated as surfaces of equal pressure or potential (specified), if the body of surface water is large in volume, so that its level is uniform and independent of changes in ground-: water flow. The uniform potential on a boundary of this type may, however, change with time due to seasonal variation in the surface water level. Other bodies of surface water, such as streams, may form boundaries with non-uniform distributions of potential that may be either constant or variable with time. A small stream, for example, might be affected by a nearby withdrawal of ground water if that withdrawal occurred at a rate of the same order of magnitude as the flow in the stream. Then the boundary condition would not be independent of the ground-water flow, that is, it would be a potential-dependent flux.

For heat transport problems associated with ATES, the most realistic boundary condition for the outer boundary of the aquifer is the temperaturedependent flux. The thermal conductivity of common geologic materials does not vary enough for the assumption of insulated conditions to be accurate. However, practical considerations when applying a particular model to an area may dictate use of the less realistic insulated, specified temperature, or specified flux conditions. These boundary conditions can be justified if they do not affect the results in the primary area of interest.

For problems in which water flows across a boundary, a difficulty occurs with the treatment of the convective term at the boundary. The temperature or concentration of this term must be specified. Usually, if flow of water is into the system modeled, the temperature or concentration is known. At an outflow point the temperature or concentration at the boundary can be used, but this may lead to some computation problems. 
FLOW IN FRACTURED, FISSURED, OR CAVERNOUS MEDIA

The mathematical equations introduced thus far have been based on a porous media assumption. Mathematical descriptions or models also exist for fractured, fissured or cavernous media. Such models are not emphasized here because fractured media are not anticipated to be extensively used in ATES. As will be discussed in a subsequent section, fractured media are not, in general, well suited for the storage of heated or cooled water. Because of this, only a brief review of modeling approaches for fractured media is presented.

It is well known that on a local or small scale, aquifers composed of fractured or cavernous media do not respond according to idealized porous media models. However, for larger-scale applications these porous media models can be used with meaningful results. Until recently flow models specifically designed for fractured or cavernous media have not received a great deal of practical attention because standard flow models have appeared to be adequate for most large scale water-supply problems. Interest in transport in fractured media, however, has stimulated the need to more accurately analyze flow in fractured media. This need has occurred because transpert models for porous media are generally inadequate to characterize transport in fractured media. Thus, fractured-media transport models are required. Because transport models must be used in conjunction with flow models, flow in fractured media is now receiving considerable attention. The results of these efforts will also be applicable to many flow in cavernous media problems.

Two general approaches for simulating flow in fractured media have been used--discontinuum and continuum. The discontinutm approach treats each fracture discretely. This requires a geometrical description of the fracture surface and its width. Discrete fracture descriptions have been coupled with continuum models for flow through porous media and elastic rock behavior (see Gale 1977, for a review of progress) and for fluid flow! and heat transport (Coats 1977). 
In the continuum approach for fractured media, the scale of the problem is assumed to be large enough such that meaningful averages of fluid pressures and velocities in fractures can be defined. For regional aquifer studies, it has been found that porous media models can be applied with good results. For problems of more local nature, an alternative approach based on a double porosity continuum has been used. The double porosity concept assumes that two porosities and permeabilities are associated with the fractured media. The primary porosity and permeability are those of the porous, though lowly-permeable, blocks that are separated by fractures. The secondary porosity and permeability are associated with fractures, where the permeability is generally high and the porosity low. It is also assumed that the porous matrix blocks act as sources (sinks) which feed (drain) the fractures with fluid. The fractures provide the main network for the movement of fluid.

Using the conceptual framework of a double-porosity aquifer, alternative numerical and analytical methods have been developed by many researchers. The most significant difference among the various models concerns the approximation of the matrix-to-fracture leakage. With minor differences two ways for treating this leakage are used--quasi-steady-state and unsteadystate. The quasi-steady-state leakage is assumed to be proportional to the difference between average heads in the fracture and matrix blocks. The proportionality function can be correlated, crudely, with the hydraulic conductivity and geometry of the porous blocks. The unsteady treatment of leakage attempts to better account for the geometry of the matrix blocks and the distribution of heads within the blocks.

Although there are several analytical and numerical models for groundwater flow in fractured rocks, other than the equivalent porous media models, few have demonstrated usefulness for practical applications. For heat storage applications, only porous media models for heat transport have been used. 
In the preceding material, physical and chemical considerations lead to partial differential equations that describe ATES. Without a solution, however, these equations are of little value. Only a simplified subset of the general equations can be solved by analytical means, and these usually describe idealized situations. In spite of their simplifying assumptions, analytical solutions are valuable tools to the ground-water hydrologist. At the very least, they can be used for a preliminary analysis to more detailed numerical modeling.

For ATES applications the two major concerns are ground-water flow and heat transport. The analytical solutions used to describe ground-water flow all assume isothermal conditions. Likewise, the heat transport solutions generally assume a very simple and known flow field.

Except for one straight-forward application, analytical solutions for ground-water flow are not described here. The number of solutions is substantial and many references already exist (see, for example, Walton (1969), Kruseman and DeRidder (1976), and Hantush (1964)). Analytical solutions for ground-water flow are used in two primary ways. They are most commonly used in the analysis of aquifer tests to determine transmissive and storage properties of the aquifer. They can also be used to predict water level changes for idealized aquifer conditions.

There are also a number of analytical solutions for various simplified forms of the solute transport equation. Examples of such solutions may be found in Ogata and Banks (1951) and Codell and Schreiber (1979). As noted, the solute transport equation is similar in form to the heat transport equation, and a solution to one can be easily modified to be a solution to the other. In general, solutions to the solute transport equation are also not discussed.

Analytical solutions are also available for analyzing aquifer tests in fractured media for a variety of aquifer conditions--confined, unconfined, quasi-steady-state leakage and unsteady leakage. Stretslova-Adams (1978) reviews and evaluates many of these solutions. Only one analytical solution for fractured media is presented in this section, that by Gringarten et al. (1975). 
The solutions discussed in this section, in general, are those analytical models that have been or can be used to examine hot or cold fluid injection. Each model is discussed separately and includes (1) problem, (2) assumptions, (3) solution and (4) possible uses. These are also summarized in Table 4.

\section{RECHARGE-DISCHARGE PAIR SOLUTION}

Problem

Many proposed ATES projects involve balanced injection and withdrawal among a group of wells. A doublet consists of two wells in the same aquifer, one an injection well and the other a pumping well. Figure 7 shows a section between a pumping and injection well and the resulting water levels in a confined aquifer.

Assumptions

The radial flow equation (35) in conjunction with the superposition principle is used to obtain the solution for steady flow among wells. The important assumptions include:

- The thickness and transmissivity are uniform over the areal extent of the aquifer.

- The areal extent is infinite.

- The aquifer is confined.

- Flow is steady.

Solution

The general solution for steady flow among any number of wells is given by Davis and Deweist (1966) as

$$
h(x, y)=\frac{1}{4 \pi T} \sum_{i=1}^{n} Q_{j} \ln \left[\left(x-x_{i}\right)^{2}+\left(y-y_{i}\right)^{2}\right]+H
$$

where

$$
\begin{aligned}
& h(x, y)=\text { the head at point } x \text { and } y \text { in a confined aquifer }(L), \\
& Q_{j}=\text { the discharge }(-) / \text { recharge }(+) \text { rate at well } i\left(L^{3} / t\right),
\end{aligned}
$$


TABLE 4. Summary of Analytical Solutions

\section{Reference}

Davis \& Deweist (1966)

Lauwerier (1955)

Gringarten et a] . (1975)

Avdonin (1964)
Problem

steady ground-water flow among wells

- linear heat transport

- linear heat transport

- Tinear heat transport

- radial heat transport

\section{Major Assumptions}

- uniform hydrologic properties

- porous medium

- convection in the receiving formation

- conductive heat loss to confining units

- convection in receiving medium

- conduction in confining bed

- finite thickness for confining bed

- conduction \& convection in receiving formation

- conductive heat loss to confining units

- single well (radial)
Usage

- evaluating wel1 spacing \& patterns

- determining groundwater flow field for heat transport solutions

- same as those given for Avdonin solution

- evaluating heat transport in fractured or fissured media

- test for numerical models

- estimating position of thermal front

- estimating heat losses to confining layers 
TABLE 4. Continued

Reference

Carslaw \& Jaeger (1959)

Gringarten \& Sauty (1975)

ת Hellström et al. (1979):

Claesson (1980)
Problem

- one-dimensional heat conduction

- heat flow between well doublet

- tilting of interface due to density differences
Major Assumptions

- one-dimensional heat conduction

- convection in the receiving formation

- conductive heat loss to confining units

- two wells

- interface separating two fluids of different density
Usage

- couple with numerical model to compute heat losses to confining layers

- estimate well spacing

- estimate production temperatures after breakthrough

- estimate tilting of thermal front 


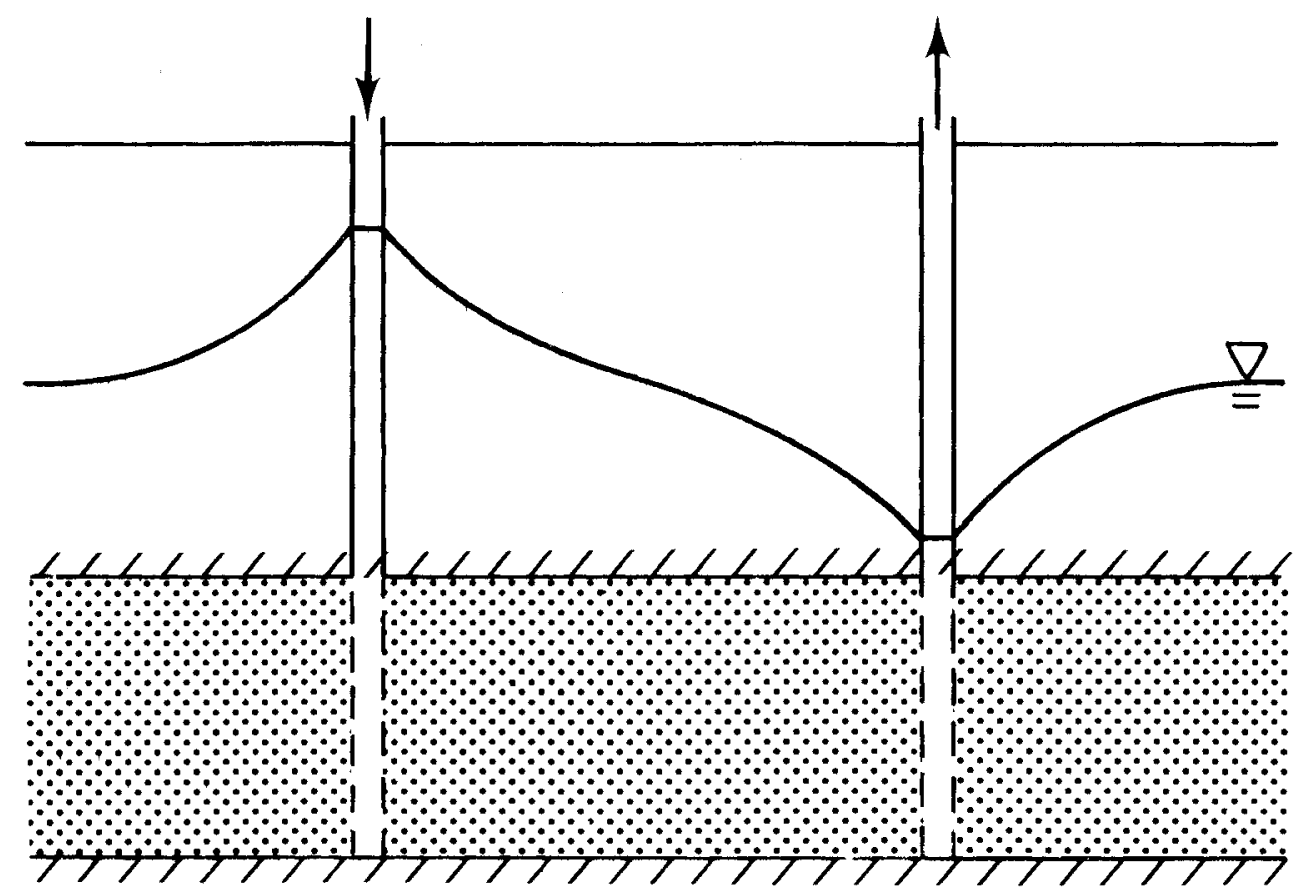

FIGURE 7. Section Showing Water Levels in a Recharge-Discharge Pair (Doublet) 
$T=$ transmissivity $\left(L^{2} / t\right)$,

$\mathrm{n} \quad=$ total number of pumping wells, and

$\mathrm{H}=\mathrm{a}$ constant determined for the particular problem, but often set to the head measured before pumping and injection.

For the special case of a doublet, Equation (43) can be written as

$$
h-H=\frac{Q}{4 \pi T}\left\{\ln \left[\left(x-x_{r}\right)^{2}+\left(y-y_{r}\right)^{2}\right]+\ln \left[\left(x-x_{d}\right)^{2}+\left(y-y_{d}\right)^{2}\right]\right\}
$$

where the subscripts $(r)$ and (d) refer to the recharge and discharge wells, respectively.

Possible Usage

This solution is useful for determining the water-level declines and buildups in planned pumping and injection wells. Because pumping and injection costs are controlled by these water levels, alternative well spacings and patterns can be evaluated before installation. The solution (44) also provides the flow field necessary to characterize heat transport in a doublet heat storage system (see Gringarten and Sauty 1975).

\section{LAUWERIER SOLUTION}

Problem

Although this solution was developed to examine hot-water flooding of an oil reservoir, it may also be used in conjunction with ATES. Heat is supplied to the formation by injection of a hot fluid through injection wells. For this special case, the flow pattern is linear, that is, where both injection and production wells are placed in a row, the two rows are parallel to each other and are considered as a line source and a line sink, respectively. The equations that are solved are

. $k_{r} \frac{\partial^{2} T_{r}}{\partial z^{2}}=\rho_{r} c_{r} \frac{\partial T r}{\partial t} \quad x>0,|z| \geq b, t>0$ 
and

$$
b \rho_{m} c_{m} \frac{\partial T}{\partial t}+b \rho_{w} c_{w} v_{w} \frac{\partial T}{\partial x}-\left.K_{r} \frac{\partial T}{\partial z}\right|_{z=b}=0 \quad x>0,-b<z<b, t>0
$$

where

$$
\rho_{m} c_{m}=(1-\phi) \rho_{s} c_{s}+\phi \rho_{w} c_{w}
$$

and

$$
\begin{aligned}
\phi & =\text { porosity } \\
c & =\text { specific heat } \\
\rho & =\text { density } \\
v & =\text { linear velocity } \\
2 b & =\text { thickness of injection formation }
\end{aligned}
$$

The subscripts refer to the overlying and underlying rock, $r$, the solid phase in the formation, $s$, water, $w$, and medium (solid and water), m. Finally, from the time $t=0$ onwards, the boundary $x=0,-b<z<b$ is kept at a constant temperature, $T_{i}>T$, by injection of water of temperature $T_{i}$ at a rate producing a velocity $v_{w}$ as to effect a convective heat transport in the $x$ - direction. The temperature at $z \cdots ! \infty$ are assumed to remain coristant.

Assumptions

A major simplifying assumption is that heat conduction in the horizontal $x$ - direction is neglected in both the injection formation and confining layeis. other assumptions include:

(1) Flow is linear.

(2) The thickness, permeability and porosity of the reservoir are uniform.

(3) Hot compressible water is injected at a constant rate.

(4) Areal extent is infinite.

(5) Horizontal convection occurs in the injection formation, whereas vertical conduction occurs in confining layers.

(6) It is assumed that the establishment of the temperature equilibrium water/solid is instantaneous.

(7) The confining layer is infinitely thick. 
Solution

Lauwerier (1955) presents a solution to Equations (45) and (46):

$$
T=T_{\mathbf{i}} \operatorname{erfc} \frac{\xi}{2 \sqrt{\theta(\tau-\xi)}} U(\tau-\xi)
$$

where $U(\xi)$ stands for the unit function: 0 for $\xi<0$ and 1 for $\xi>0$. Other terms are defined as

$$
\xi=\frac{x K_{r}}{b^{2} \rho_{w}{ }^{c} w^{v} w} ; \quad \theta=\frac{\rho_{m} c_{m}}{\rho_{r} c_{r}} ; \tau=\frac{t K_{r}}{b^{2} \rho_{m} c_{m}}-.
$$

Possible Usage

The solutions presented by Avdonin (and discussed later) are more general and usually should be used instead of that given by Lauwerier. Equation (47) is sometimes used in place of Avdonin's solution because it is easy to evaluate. Lauwerier's solution has been used for comparison with numerical models. It is interesting to note that (1) having a large thickness produces a sharp front and (2) a high velocity also produces a sharp front. To smear the front (not desired for ATES), decrease (1) and/or (2).

It is interesting to note that Grisak and Pickens (1980) have used this basic solution to describe solute transport through fractured media with matrix diffusion.

GRINGARTEN ET AL. SOLUTION

Problem

To consider heat extraction from a dry geothermal area, Gringarten et a1. (1975) posed a mathematical problem similar to that of Lauwerier. The primary difference in the two problems is that in the specifications of the boundary condition in the z-direction. Whereas Lauwerier assumed that boundary was at infinity, Gringarten et al. consider a finite boundary with a no-heat flow condition. The governing equations are (45) and (46). 
Assumptions

The same as those for the Lauwerier solution, except that

$$
\frac{\partial T}{\partial z}=0 \quad \text { at } z=\ell
$$

where $\ell$ is the position of a symmetry or insulation boundary.

Solution

The solution given by Gringarten et al. (1975) is posed in terms of a complicated Laplace transform that can be inverted (evaluated) numerically. The interested reader is referred to the original publication.

Possible Usage

This solution is particularly applicable to aquifers comprised of a system of parallel fractures. The solution can be used to evaluate the temperatures in the fracture and surrounding matrix. In this case, the fracture zone is the "aquifer" and the rock matrix is the "confiring bed". Such an analysis generally will show that fractured systems are not desirable for ATES because heat becomes too dispersed.

\section{AVDONIN SOLUTION}

Problem

The problem is to determine the temperature field in a formation during the injection of a hot incompressible fluid through a single well. Both conduction and convection are considered in the formation whereas conductive thermal heat loss is considered in the rocks overlying and underlying the formation. Solutions are presented for both linear and radial flow.

The linear flow of hot compressible fluid through a confined aquifer may be described by

$$
k_{r} \frac{\partial^{2} u}{\partial z^{2}}=\rho_{r} c_{r} \frac{\partial u}{\partial t} \quad z>0, t>0
$$


and

$$
k_{m} \frac{\partial^{2} u}{\partial x^{2}}-v_{w} \rho_{w} c_{w} \frac{\partial u}{\partial x}+\frac{2}{b} k_{r} \frac{\partial u}{\partial z}=\rho_{m} c_{m} \frac{\partial u}{\partial t} \quad z=0, x>0, t>0
$$

subject to the initial conditions $u(x, 0)=0$ for $x>0$ and the constant temperature boundary condition at the injection well,

$$
u(0, t)=1, \quad z=0, \quad t>0 ; \quad \begin{array}{ll}
\text { imit } \quad u=0 \\
\\
x^{2}+z^{2} \rightarrow \infty
\end{array}
$$

where $u=\left(T-T_{0}\right) /\left(T_{1}-T_{0}\right)$ is the normalized temperature, $T_{0}$ is the overburden (underburden) temperature and the initial aquifer temperature, $T_{1}$ is the temperature of the injection fluid, $c$ is heat capacity, and $v$ is average intergranular velocity. The subscripts refer to the rock $r$, water $w$, and medium (rock and water) $\mathrm{m}$.

\section{Assumptions}

The assumptions invoked in the solution of the above equations involve:

(1) Equation parameters such as porosity, heat capacity, thermal conductivity, thickness, and density are constant.

(2) There is also a uniform velocity in the formation. That is, the injection rate is uniform and steady-state flow conditions exist in the formation. This velocity is a result of the injection only, and does not consider regional flow.

(3) The areal extent of the formation is infinite.

(4) Convection and conduction in the formation are negligible in the vertical direction.

(5) Convection does not occur and conduction is negligible in the horizontal direction in the rocks overlying and underlying the formation.

(6) The extent of the confining bed is infinite. 


\section{Solution}

Avdonin (1964) presents an analytical solution for Equations (48) and (49):

$$
\begin{aligned}
& u(x, \tau)=\frac{x}{(\pi \tau)^{1 /},} \int_{0}^{1}\left\{\exp \left[-\left(s \gamma(\tau)^{1 / 2}-\overline{2 s(\tau)^{1 / \tau}}\right)^{2}\right]\right. \\
& \left.\quad \operatorname{erfc}\left(\frac{\alpha s^{2}(\tau)^{1 / 2}}{2\left(1-s^{2}\right)^{1 / 2}}\right)\right\} \frac{d s}{s^{2}}
\end{aligned}
$$

where

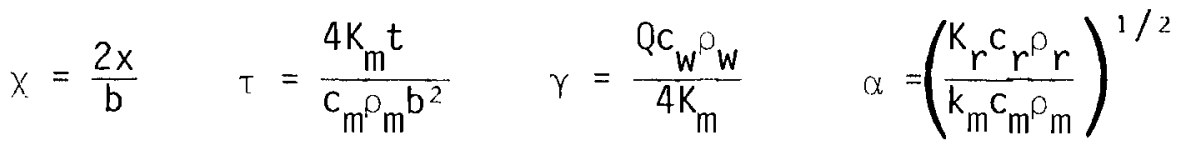

and $Q$ is the injection flow rate. (In the original reference, $\gamma$ is defined with 8 in the denominator; this appears to be a typographical error).

Avdonin also presents an analytical solution for a problem similar to Equations (48) and (49) involving radial flow:

$$
u(\omega, \tau)=\frac{1}{\Gamma(\nu)}\left[\frac{\omega^{2}}{4 \tau}\right]^{\nu} \int_{0}^{1}\left\{\exp \left(-\frac{\omega^{2}}{4 \tau s}\right) \operatorname{erfc}\left(\frac{\alpha s \sqrt{\tau}}{2 \sqrt{1-s}}\right)\right\} \frac{d s}{v+1}
$$

where

$$
v=\frac{Q c_{w} \rho_{w}}{4 \pi b K_{m}}, w=\frac{2 r}{b} \text {, }
$$

$r$ is the radial coordinate, and all other terms are defined as in Equation (50).

\section{Possible Usage}

Equations (50) and (51) may be used to test numerical solutions. In addition, Equation (51) may be used to estimate the location of the thermal front's location from an injection well. This, in turn, may be used to help locate monitoring wells. Also given in the solution is an estimate of heat losses to confining layers. 


\section{ONE-DIMENSIONAL HEAT CONDUCTION}

Problem

An estimate to conductive heat loss to confining units may be obtained by solving the following equation

$$
K_{m} \frac{\partial^{2} T}{\partial z^{2}}=\rho_{m} c_{m} \frac{\partial T}{\partial t}
$$

which is the one-dimensional, heat conduction equation where

$$
\begin{aligned}
& \mathrm{K}_{\mathrm{m}}=\text { medium thermal conductivity, }\left(\mathrm{MLt}^{-3} \mathrm{~T}^{-1}\right), \\
& \rho_{\mathrm{m}}=\text { medium density, }\left(\mathrm{ML}^{-3}\right), \text { and } \\
& \mathrm{c}_{\mathrm{m}}=\text { medium heat capacity, }\left(\mathrm{L}^{2} \mathrm{t}^{-2} \mathrm{~T}^{-1}\right) .
\end{aligned}
$$

If it is assumed that the temperature distribution is initially at steadystate, then

$$
\frac{\partial^{2} T_{0}}{\partial z^{2}}=0
$$

where $T_{0}$ is the initial temperature distribution. Subtracting Equation (53) from (52) gives

$$
K_{m} \frac{\partial^{2} T^{\star}}{\partial Z^{2}}=\rho_{m} c_{m} \frac{\partial T^{\star}}{\partial \bar{t}}
$$

where $T^{*}=T-T_{0}$. Equation (54) is subject to the following initial and boundary conditions:

$$
\begin{aligned}
& T^{\star}(z, 0)=0, \\
& T^{\star}(0, t)=0, \text { and } \\
& T^{\star}(\ell, t)=T^{\prime}
\end{aligned}
$$

where $T^{\prime}$ is a constant temperature at some distance $\ell$. 


\section{Assumptions}

This problem may also be thought of as a subset to the Avdonin problems in that it represents only the conductive leakage portion to confining layers. Assumptions include:

(1) Permeability is low and convective flow may be neglected.

(2) The effects of heat conduction in directions other than the z- direction are negligible.

(3) $K_{m}, \rho_{m}$ and $c_{m}$ are constant.

Solution

This particular problem has an analytical solution of the form (Carslaw and Jaeger, 1959):

$$
T^{*}=T^{\prime} \frac{z}{\ell}+\frac{2}{\pi} \sum_{n=1}^{\infty} \frac{T^{\prime}(-1)^{n}}{n} \sin \frac{n_{\pi i z}}{\ell} \exp \left(-\frac{K_{m}}{\rho_{m} c_{m}} \frac{n^{2} \pi^{2} t}{\ell, 2}\right)
$$

\section{Possible Usage}

Equation (55) may be coupled with a numerical model of the injection formation in ATES to compute conductive heat losses to confining layers without having to treat the confining units as part of the grid for the numerical portion. That is, based on the step function in temperature at the reservoir-confining layer boundary and the solution given by Equation (55), the heat flux can be determined using Fourier's Law. If the step function at the boundary changes with time, however, a numerical solution of Equation (54) may be more appropriate.

\section{GRINGARTEN AND SAUTY SOLUTION}

\section{Problem}

The problem is to calculate the movement of a therma? front between a well doublet. Water is withdrawn from one well, heated or chilled using an energy source, and reinjected back into the aquifer via the second well. 
The equations, as discussed by Gringarten and Sauty (1975), which describe this problem are very similar to those presented in the section on Lauwerier solution, and will not be repeated. The main difference is that here the equations are used to describe the water temperature within a streamchannel.

\section{Assumptions}

The assumptions are as follows:

(1) The aquifer is of infinite extent, horizontal, and of uniform thickness. It is confined above and below by layers that are impermeable and of infinite vertical extent.

(2) Flow is steady, with the injection rate constant and equal to the production rate. All wells are fully penetrating, with the flow field due to the recharging and discharging wells superimposed on the natural flow system.

(3) Initially, the water and rock in all layers are at the same temperature. At time $t=0$, the temperature of the injected water is set equal to $T_{0}$, and maintained constant thereafter. Thermal equilibrium is assumed to take place instantaneously between rock and water.

(4) There is no heat transfer by conduction in the horizontal directions within the aquifer or confining layers. All heat transport is by vertical conduction above and below the aquifer, and forced convection in the horizontal direction within the aquifer.

(5) Equation parameters are assumed constant, and differences in viscosity between injected and native water are neglected.

\section{Solution}

In general, the solution is similar to Lauwerier's. If the streamlines leaving a recharge we11 reach a discharge we11, the water temperature at the discharge well within an elementary streamchannel is given by

$$
\frac{T_{0}-T^{\psi}}{T_{0}-T_{i}}=\operatorname{erfc}\left\{\frac{d\left(S_{\max } / D^{2}\right)}{d(\psi / Q)}\left[\lambda\left(t_{D}-\frac{d\left(S_{\max } / D^{2}\right)}{d(\psi / Q)}\right]^{-1 / 2}\right\}\right.
$$

where

$$
\lambda=\frac{\rho_{w}{ }^{c} w^{\rho}{ }^{c} m}{K_{r} \rho_{r} c_{r}} \quad \frac{Q h}{D^{2}} ; \quad t_{D}=\frac{\rho_{w}{ }^{c} w}{\rho_{m} c_{m}} \frac{Q t}{D^{2} h}
$$


and where

$T_{0}$ is the initial and confining layer temperature,

$\mathrm{T}^{\psi}$ is the water temperature within a streamchannel,

$T_{j}$ is the injection temperature,

$\mathrm{S}_{\max }$ is the total streamchannel area between the two wells,

$Q$ is the injection rate,

$D$ is some characteristic length,

$\psi$ is the streamline,

$h$ is the aquifer thickness,

$\rho$ is density,

$c$ is specific heat, and

$K$ is thermal conductivity.

As before, the subscripts refer to the rock, $r$, water, $w$, and medium, $m$. $\frac{d\left(S_{\max } / D^{2}\right)}{d(\psi / Q)}$ is the dimensionless derivative of the tntal streamchannel area with respect to the streamfunction. Note that heat transfer between the aquifer and confining layers is negligible for $\lambda \geq 10^{4}$.

The water temperature at the recharge well at a given time $t_{D}$ is obtained by integrating the right side of Equation (56) with respect to $\psi$ for all streamchannels that have reached the discharge well at $t_{D}$ (Gringarten and Sauty 1975):

$$
\frac{T_{0}-T}{T_{0}-T_{i}}=\operatorname{erfc}\left\{\frac{d\left(S_{\max } / D^{2}\right)}{d(\psi / Q)}\left[\lambda\left(t_{0}-\frac{d\left(S_{\max } / D^{2}\right)}{d(\psi / Q)}\right]^{-1 / 2}\right\} d\left(\frac{\psi}{Q}\right)\right.
$$

This result is general where $T$ can be evaluated if the flow field is known. The procedure requires integration of the effects of all streamchannels in order to determine the temperature of the water being pumped. The location of the streamchannels for the most ideal case can be determined from Equation (44). 


\section{Possible Usage}

For the doublet problem, the characteristic length $D$ in Equation (57) is the distance between the two we11s. Gringarten and Sauty show that by using information from Equation (57) the following may be computed: (1) the spacing between the two wells in order not to have any change in temperature at the production well during a specified period, and (2) the temperature change at the production well after breakthrough. The use of this solution is discussed further in Tsang et al. (1977a) and Lippmann and Tsang (1980).

\section{TIL.TING FRONT SOLUTION}

\section{Problem}

A vertical front dividing waters of different temperatures that may result from a fully-penetrating injection well is unstable because of the lower density of the warmer water. The thermal front will tilt so that the warmer water eventually ends on top. This and other thermal stratification effects, which entail undesired heat losses, were examined by Hellström et al. (1979) and Claesson (1980).

\section{Assumptions}

The assumptions include the following:

(1) Darcy's equation is valid,

(2) Compressibility effects are neglected,

(3) The aquifer is bounded by impermeable layers and injection/extraction wells,

(4) Density and viscosity are functions of temperature, but uniform in each fluid,

(5) Aquifer properties such as porosity and thickness are uniform.

\section{Solution}

Solutions are presented for (1) infinite strip, (2) semi-infinite strip with impermeable left boundary, (3) semi-infinite strip with free flow in a well along the left boundary, (4) circular disc, (5) cylindrical case, and (6) infinite strip with thermal front thickness uniform. For the sake of brevity, we only present the solutions of cases 5 and 6 ; for the other 
solutions, see Hellström et a1. (1979).

For the cylindrical case, the horizontal ground-water flow across the front, $q_{f}(z)$ is given by,

$$
\begin{aligned}
q_{f}(z)= & \sqrt{\frac{k^{T}}{k}} q_{0} \cdot \frac{4}{\pi} \sum_{n=0}^{\infty} \frac{(-1)^{n}}{2 n+1} \cdot \sin \left[\frac{(2 n+1) \pi z}{H}\right], \\
& {\left[\frac{\mu_{1}}{\mu_{0}+\mu_{1}} \cdot \frac{I_{0}\left(\gamma_{n}\right)}{I_{1}\left(\gamma_{n}\right)}+\frac{\mu_{0}}{\mu_{0}+\mu_{i}} \cdot \frac{K_{0}\left(\gamma_{n}\right)}{K_{1}\left(\gamma_{n}\right)}\right], }
\end{aligned}
$$

where

$$
\begin{aligned}
k & =\text { horizontal permeability, } \\
k^{\prime} & =\text { vertical permeability, } \\
z & =\text { vertical cartesian coordinate, } \\
H & =\text { height of aquifer stratum, } \\
\mu_{i} & =\text { dynamic viscosity for water at temperature } T_{i}, \\
q_{0} & =\text { characteristic buoyancy flow } \\
& =k\left(\rho_{0}-\rho_{1}\right) g /\left(\mu_{0}+\mu_{1}\right) \\
g & =\text { gravity, } \\
\rho_{i} & =\text { density of water at temperature } T_{i}, \\
\gamma_{n} & =(2 n+1) \pi \sqrt{k^{\prime}} \cdot L / H, \\
L & =\text { thickness of warm aquifer region, }
\end{aligned}
$$

and $K_{n}$ and $I_{n}$ are the modified Bessel functions.

For an infinite strip with diffuse thermal front, the solution is

$$
\begin{gathered}
q_{f}(z)=\sqrt{\frac{k^{\prime}}{k}} q_{0} \frac{4}{\pi} \sum_{n=0}^{\infty} \frac{(-1)^{n}}{2 n^{n}+1}\left\{1-\exp \left[-(2 n+1) \pi \sqrt{\frac{k^{\prime}}{k}} \frac{D}{2} / H\right]\right\} / \\
(2 n+1) \pi \sqrt{k^{\prime}} \frac{D}{2} / H \cdot \sin [(2 n+1) \pi z / H]
\end{gathered}
$$


where the new term, $D=$ thickness of the thermal front. The flow $a_{f}(z)$ refers to the middle of the thermal front region. For $\operatorname{large}_{\sqrt{\frac{k}{k}}} \frac{\mathrm{D}}{\mathrm{H}}$, a good approximation is

$$
q_{f}(z) \because 2 q_{0} z / D
$$

\section{Possible Usage}

These solutions may be used to estimate the tilting of the thermal front. Hellström et al. (1979) also analyze buoyancy and forced convection by use of superposition. 
NUMERICAL SOLUTIONS

Numerical models provide the most general tool for the quantitative analysis of ATES applications. They are not subject to many of the restrictive assumptions required for analytical solutions. In spite of the flexibility of numerical models, their mathematical basis is actually less sophisticated than that of the analytical methods.

To develop a numerical model of a physical system (in our case, an aquifer), it is first necessary to understand how that system behaves. This understanding takes the form of laws and concepts. As presented, these concepts and laws are then translated into mathematical expressions, usually partial differential equations, with boundary and initial conditions. Numerical methods provide a means for solving these equations in their most general form.

Numerical solution normally involves approximating continuous partial differential equations with a finite number of equations at discrete points in time and space. Thus, the region and time period of interest are divided in some fashion, resulting in an equation or set of equations for each subregion and time step. These discrete equations are combined to form a system of algebraic equations that must be solved for each time step. Finite-difference and finite-element methods are the major numerical techniques used in ground-water applications. The important components and steps of model development for the two alternative methods are shown in Figure 8 .

A general discussion on finite-difference and finite-element techniques is given in Appendix $B$. In this section, pertinent numerical models used for ATES are reviewed. In addition, several models are discussed in detail.

Comprehensive reviews on models used in geothermal applications have been presented by Cheng (1978), Pinder (1979) and Mercer and Faust (1979b). Some of the models discussed in these references will also be discussed here. In addition to these references, Tsang et al. (1980a) present a review on ATES that also contains numerical models. 


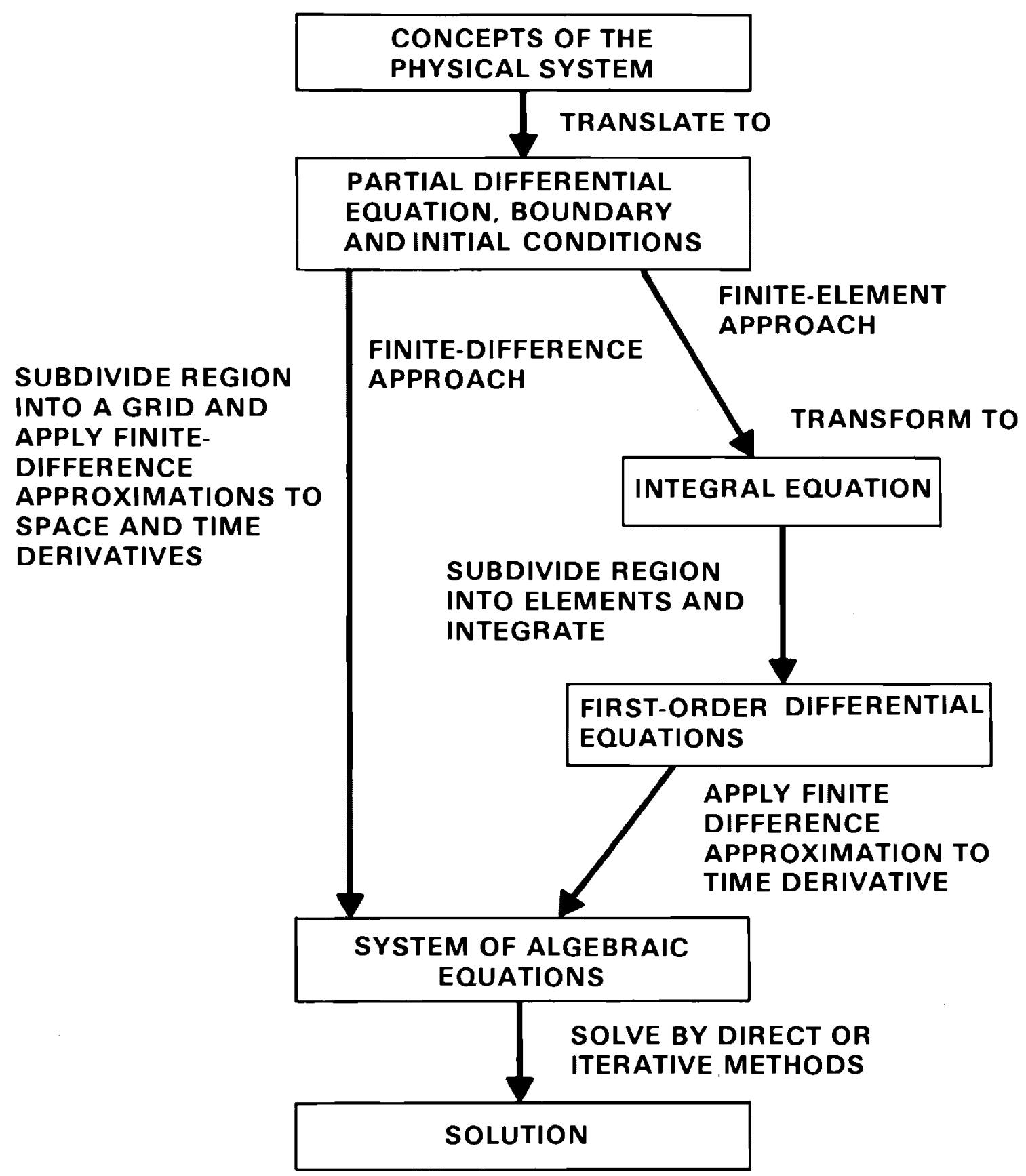

FIGURE 8. Generalized Model Development by Finite-Difference and Finite Element Methods (from Faust and Mercer 1980) 
Table 5, extended from Tsang et a1. (1980a), summarizes current modeling studies in ATES. This table is by no means complete, but does indicate the level of effort for numerical modeling of heat transport. Several models are discussed in detail. They were chosen because they are generally well documented and have been used to examine several heat transport problems. They were also selected because they represent a fairly complete sampling of available codes. In addition to using approximating techniques such as finite difference, integrated finite difference and finite element, the codes treat heat flow as well as consolidation, solute transport and twophase flow. Each code is discussed separately and the following topics are covered: (1) governing equations, (2) assumptions, (3) solution, and (4) application.

$\underline{\mathrm{CCC}}$

Governing Equations

The numerical model CCC (Conduction-Convection-Consolidation) is described in Mangold et a1. (1980). It solves for flow and heat transport in a liquid saturated medium, and computes one-dimensional consolidation. The governing equations employed in the model are the basic mass and energy balance laws. The mass flow equation can be written in integral form as

$$
\frac{d}{d t} \int_{V} \phi \rho c_{T} p d V=-\int_{A} \rho \bar{v} \cdot \bar{n} d A+\int_{V} \rho R d V .
$$

Equation (60) applies to any control element of volume $V$ and surface area $A$. The terms in Equation (60) are similar to those presented in Equation (13), except that it is now written in integral form. New symbols include

$$
\begin{aligned}
& c_{T}=\text { total matrix compressibility } \\
& \bar{n}=\text { outward unit normal to the surface bounding } v .
\end{aligned}
$$


TABLE 5. Modeling Studies in ATES

(extended from Tsang et al. 1980a)

Research Institute

Royal Institute of Technology

Stockholm, Sweden

(Pinder et al. 1980; Voss et al. 1981)

Lund University, Sweden

(Hellströn 1978; Claesson et al. 1978)

Technical University of Denmark, Denmark (Qvale 1978; Reffstrup 1979)

University of Neuchâtel, Switzerland

(Mathey 1977; Mathey and Manjos 1978)

Institut de Production d' Energie de 1 'École Polytechnique Fédérale de Lausanne, Switzerland

(Joos 1978)

École des Mines de Paris, France (deMarsily 1978)

Bureau des Recherches Géologiques et Minieres (BRGM), France

(Gringarten et a 1. 1977)

Yamagata University, Japan

(Yokoyama et al. 1980)

U.S. Geological Survey, United States

(Papadopulos and Larson 1978)
Project

One- and two-dimensional finite element model

Two-dimensional, doublet, semianalytical model; two-dimensional finite-difference program developed to study storage in eskers

One- and two-dimensional finite element models; study of using compensation wells for countering regional flow

Two- and three-dimensional

finite element models

Three-dimensional finite element mode 1

Two-dimensional, radial, finitedifference model; two- and three-dimensional finite element models

Layered two-dimensional finitedifference mode 7

Two-dimensional finite-difference model

SWIP mode1, based on a finite-difference scheme, used to model the Auburn experiment 


\section{TABLE 5. Continued}

Research Institute

U.S. Geological Survey, United States

(Faust and Mercer 1979b)

Lawrence Berkeley Laboratory, United States Two- and three-dimensional inte-

(Mangold et al. 1980)

Texas A\&M University, United States

(Grubaugh and Reddel1 1980)

Dames \& Moore, United States

(Huyakorn, written comm. 1980)

University of Kansas, United States

(Willhite et al. 1974)

Pacific Northwest Laboratories, United States

(Gupta et al. 1980)
Project

Three-dimensional finite-difference model (GEOTHER) grated finite-difference model (CCC)

Three-dimensional finite element model

Two- and three-dimensional finite element mode1s (GREASE2 \& GREASE3)

Three-dimensional finite-difference model

Multi-dimensional finite element model (CFEST) 
The energy equation can also be written in integral form as

$$
\begin{gathered}
\frac{d}{d t} \int_{V}\left(\phi \rho c_{p}+(1-\phi) \rho_{r} c_{p r}\right) T d V=\int_{A} K_{m} \bar{\nabla} T \cdot \bar{n} d A \\
-\int_{A} \rho \bar{q} c_{p} \delta T \cdot \bar{n} d A+\int_{V} R c_{p} T^{\prime} d V
\end{gathered}
$$

where in the convective term, $\delta T$ denotes the difference between the interface temperature and the temperature of the node.

Equations (60) and (61) are nonlinear in that the parameters $\rho, k, \mu$, $k_{m}$ and $c_{p}$ depend on pressure and/or temperature. Furthermore, the parameters $\phi, c_{T}$ and $k$ are stress dependent. These relationships are described using the one-dimensional consolidation theory of Terzaghi (1925).

The basic concept in Terzaghi's theory is the relationship between the effective stress $\sigma^{\prime}$ and the pore pressure, p. For a saturated medium, this may be expressed as

$$
\sigma^{\prime}=\sigma_{n}-p
$$

where $\sigma_{n}$ denotes the normal stress (overburden). The consolidation behavior of each material is described by

$$
\phi=e /(1+e) .
$$

Porosity, in turn, may be used to compute the total matrix compressibility by

$$
c_{T}=\beta_{p}+\alpha / \phi(1+e)
$$

where $\beta_{p}$ is the compressibility coefficient of the fluid, $\alpha$ is the intergranular vertical compressibility, and $e$ is the void ratio. Note that the approximations (63) and (64) are slightly different than those used in Equations (15) and (16). 
Finally, permeability changes are computed using

$$
k=k_{0} \exp \left[2.303\left(e-e_{0}\right) / C_{k}\right]
$$

where $k_{0}$ and $e_{0}$ are arbitrary reference values of the permeability and void ratio, respectively, and $C_{k}$ is the slope of the best fitted line of void ratio versus $\log k$.

Assumptions

The major assumptions according to Mangold et al. (1980) are:

(1) Darcy's equation adequately describes fluid movement.

(2) The rock and fluid are in thermal equilibrium.

(3) Energy changes due to the fluid compressibility, acceleration and viscous dissipation are neglected.

(4) One-dimensional consolidation theory is adequate to describe vertical deformation.

Solution

Equations (60) and (61) are solved using the integrated finitedifference method, which is discussed in Appendix B. The basic idea is to solve the equations directly without reducing them to point equations. The volume $V$ is selected to be a multi-faceted sphere (a polygon in two space dimensions) where the flux across each face is approximated using a finitedifference approximation of these first order terms (Pinder 1979). To evaluate volume boundary temperatures, upstream weighting is used.

The pressure and energy equations are solved alternatively by interlacing them in time. Since in general the pressure changes much more rapidly than the temperature, much smaller time steps are used in the flow cycles than in the energy cycles. A detailed explanation of the solution technique, called mixed explicit-implicit iterative scheme, can be found in Narasimhan et al. (1978).

\section{Applications}

A summary of CCC's options is presented in Table 6 . These options have been extensively tested (Tsang et al. 1978a) by comparison with 


\section{TABLE 6. Summary of Model Characteristics}

and Options for CCC Code

Major Assumptions:

Approximating method:

Solution technique:

Geometry:

Options:

Boundary conditions:
- porous media

- single phase flow

- one-dimensional consolidation

- integrated finite-difference

- similar to successive over-relaxation

- 1-,2-, or 3-dimensional cartesian

- cylindrical

- spherical

- steady or transient flow

- heat transport

- consolidation

- heterogeneous and/or anisotropic media

- specified value

- specified flux 
analytical solutions, such as those by Avdonin (1964) and Gringarten and Sauty (1975). In addition, the code has been used in a number of generic studies (Tsang et al. 1976) as well as field applications. The model was applied to the two cycles of injection, storage and production performed by Auburn University, which will be discussed in detail in the field application section. The model has also been used to examine the physical feasibility of using underground aquifers as a storage system for hot water from solar energy collectors (Tsang et al. 1977a and Tsang et al. 1978b) and to examine ATES in eskers (He11ström 1979).

In addition to these studies, Tsang (1980) used a simplified model to examine the energy recovery factor, which he defines as the ratio of energy recovered to energy stored, as a function of aquifer properties and storage parameters. In this study, he assumes no buoyant flow and steady-state fluid flow in an infinite, uniform aquifer. The recovery factor was calculated using differirg values of aquifer thickness, storage volume, aquifer and aquitard thermal conductivity, caprock thickness, cycle time period, velocitydependent dispersion, and the number of cycles. In a11 cases, equal volumes of fluid are injected and produced. Some of the results from this study are shown in Figures 9-12. According to Tsang,

Figure $\{9\}$ shows the energy recovery factor as a function of thermal radius, Rth, and aquifer thickness, $H$. Each dashed line traces the recovery factor for a given fluid volume. There is an optimal value of $\mathrm{Rth} / \mathrm{H}$ which yields the maximum recovery factor for each volume. Generally, the recovery factor is a much more sensitive function for small values of $R_{t h}$ and $H$ than for large values. Figure $\{10\}$ shows the recovery factor as a function of volume for a series of values of aquifer thickness, $H$. The recovery factor increases rapidly at first, then levels off. Figure $\{11\}$ shows the recovery factor as a function of the aspect ratio, $\mathrm{R}_{\mathrm{th}} / \mathrm{H}$, for a series of aquitard to aquifer thermal conductivity ratios. As this ratio decreases, the aspect ratio which yields the maximum recovery factor increases. Figure 12$\}$ shows energy recovery as a function of the time period of a single injection-storageproduction cycle for several different injected volumes. The aquifer thickness for each volume is such that the aspect ratio is optimal. Lines "a" show the results for a cycle with no storage period, i.e., production begins as soon as injection ends. In this figure, injection and production periods are equal. We have found that varying the relative injection and production periods for a given storage period has only a minor effect on the recovery factor. Lines labeled "b" show 


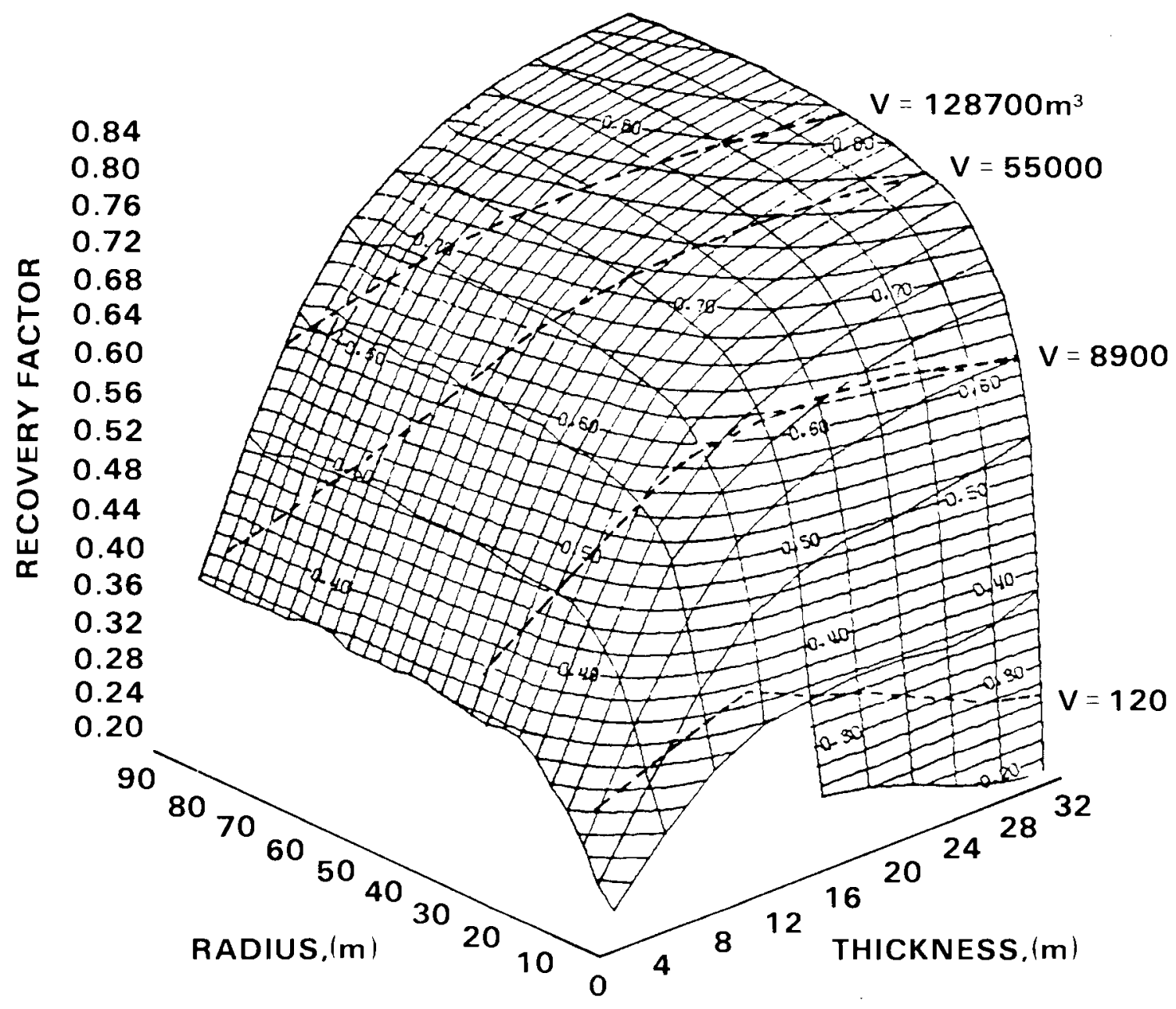

FIGURE 9. The recovery factor as a function of aquifer thickness and storage radius. The latter is defined as the radius reached by the storage temperature front at the end of injection period. (from Tsang 1980). 


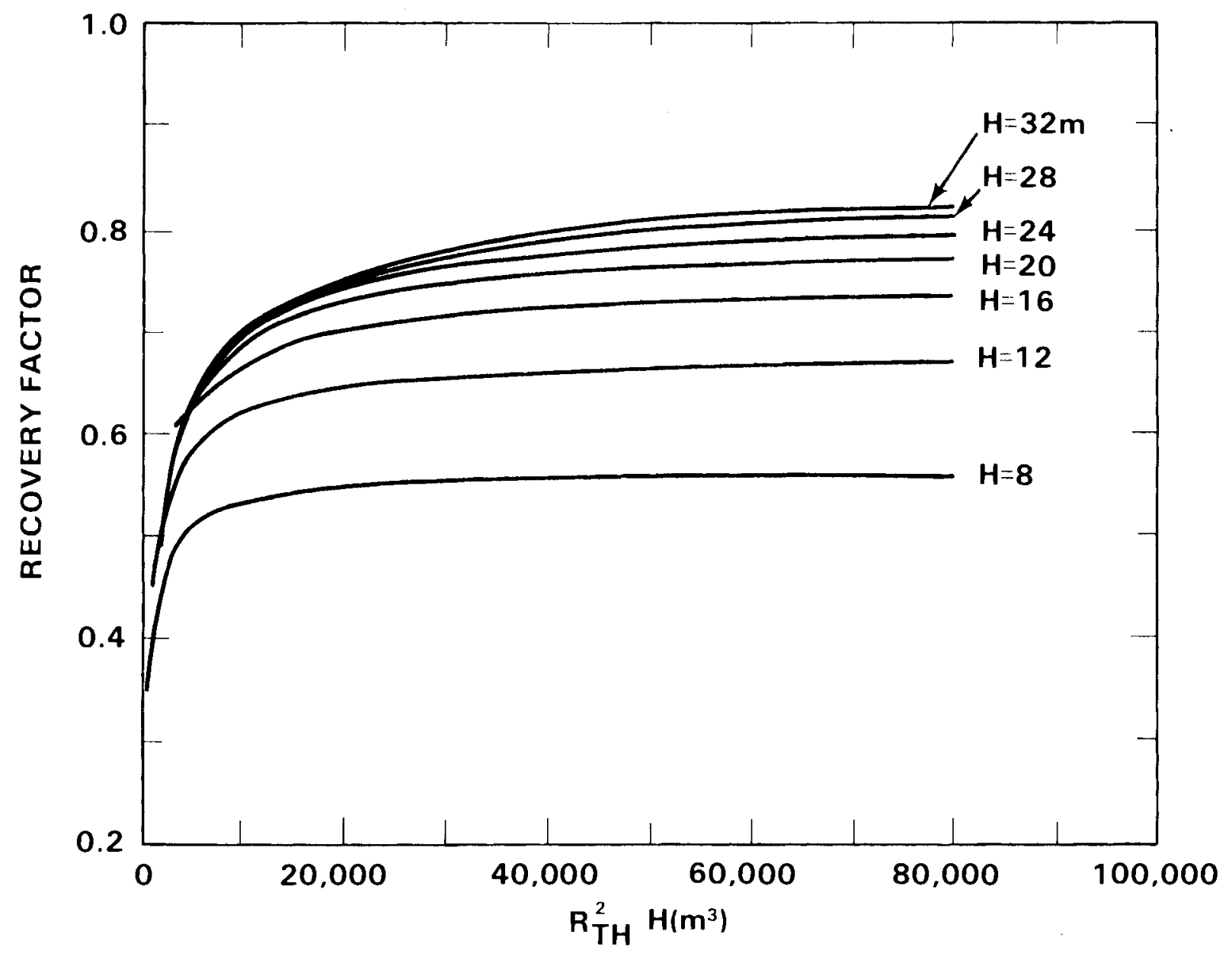

FIGURE 10. The recovery factor as a function of storage volume, implicitly given by $\mathrm{R}^{2}$ thH for different values of aquifer thickness $H$ (from Tsang 1980). 


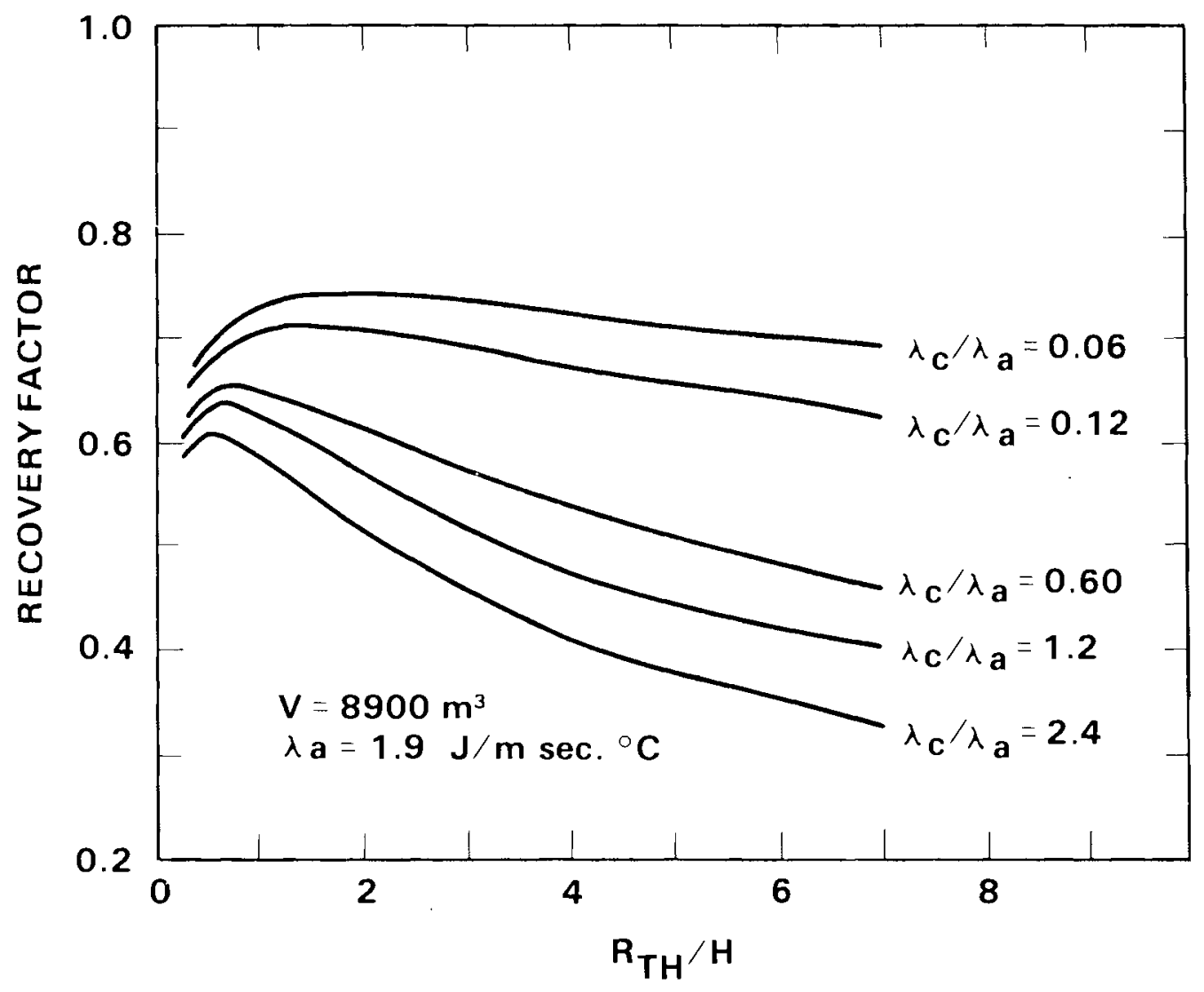

FIGURE 11. The recovery factor as a function of aspect ratio, storage radius over aquifer thickness (Rth/H), for different aquifer and aquitard thermal conductivities ( $\lambda$ a and $\lambda_{c}$ ) (from Tsang 1980). 


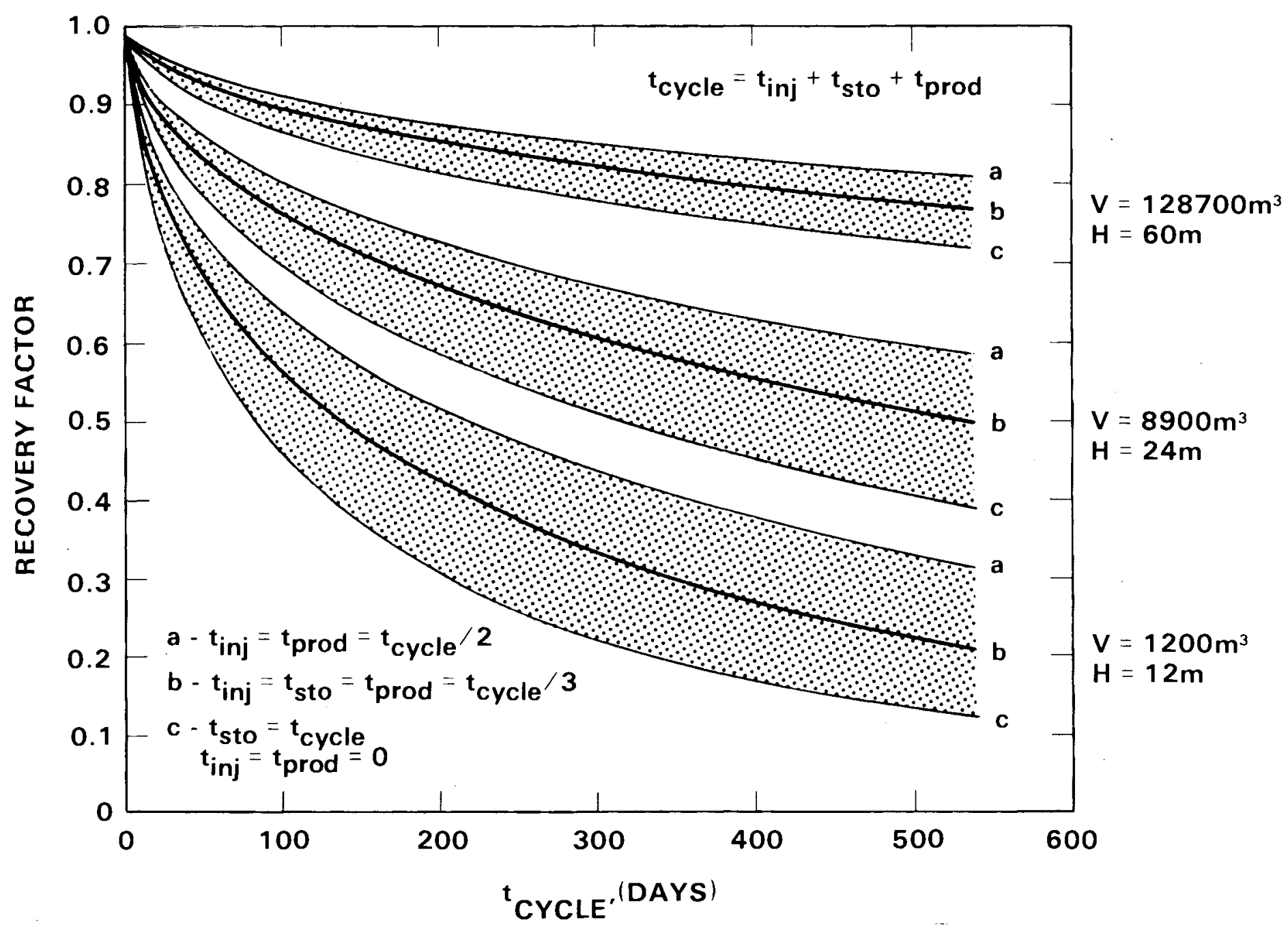

FIGURE 12. The Recovery Factor as a Function of Cycle Time Periods ( $t_{\text {cycle }}$ ) (from Tsang 1980) 
the results for a cycle with equal injection, storage, and production periods. Lines labeled " $c$ " show the results for the hypothetical cycle that is all storage period, the hot water being instantly injected and later instantly produced. This represents the limit of very short injection and production periods.

SWIP

Governing Equations

The numerical model SWIP (Survey Waste Injection Program) is described in INTERCOMP (1976). It was originally developed for the U.S. Geological Survey to be used for waste injection problems, and as such, not only considers flow and heat transport in a liquid-saturated porous media, but also considers solute transport.

The equations are essentially the same as those presented in the mathematical description section. More specifically, the flow equation is Equation (13), whereas the heat transport equation is (36), only with some parts of the equation in terms of enthalpy (heat content). The solute transport equation is Equation (42).

In addition to these equations, which describe reservoir conditions, SWIP couples reservoir and wellbere models. The wellbore model simulates the injection (or production) well conditions and provides the boundary conditions for the reservoir model. The energy losses in the well are calculated to obtain the bottom-hole pressures and temperatures. Equations describing the wellbore model may be found in the original reference.

Assumptions

The major assumptions include (INTERCOMP 1976):

(1) Three-dimensional, transient, laminar (Darcy) flow.

(2) Fluid density can be a function of pressure, temperature and concentration. Fluid viscosity can be a function of temperature and concentration.

(3) Miscibility of the injected wastes with the in-place fluids.

(4) Aquifer properties vary with position.

(5) Hydrodynamic dispersion is described as a function of fluid velocity. 
(6) The energy equation can be described as enthalpy in-enthalpy out = change in internal cenergy of the system. This is rigorous except. for kinetic and potential energy which have been neglected.

(7) Boundary conditions allow natural water movement in the aquifer, heat losses to the adjacent formation, and the location of injection, production and observation points anywhere within the system.

(8) Thermal equilibrium exists.

Solution

The equations are solved by dividing the region of interest into a rectilinear grid and developing finite-difference approximations for the grid. This method is discussed in more detail in Appendix B. The resulting difference equations are nonlinear because, in general, the fluid properties are functions of the dependent variables.

Special attention is given to numerical dispersion and oscillation. For problems dominated by convection, one of these two difficulties will generally occur depending upon the numerical approximation and solution. Basically, for finite-difference approximations, the problem can be described as (INTERCOMP 1976):

(1) A first order correct finite-difference approximation to the time or convective (first order) space derivative results in second order truncation error which is virtually identical to the physical dispersion.

(2) A second order correct finite-difference approximation eliminates the term identical to physical dispersion, but introduces a time step and block size restriction for obtaining good results, that is, overshoot and undershoot.

To cope with these problems, the user has the option in SWIP to choose from among several difference forms:

(1) Backward-In-Space (BIS), Backward-In-Time (BIT)

(2) Backward-In-Space (BIS), Central-In-Time (CIT)

(3) Central-In-Space (CIS), Backward-In-Time (BIT)

(4) Central-In-Space (CIS), Central-In-Time (CIT)

These are summarized in Table 7 along with truncation error forms and stability considerations. Equivalent truncation error forms or stability 


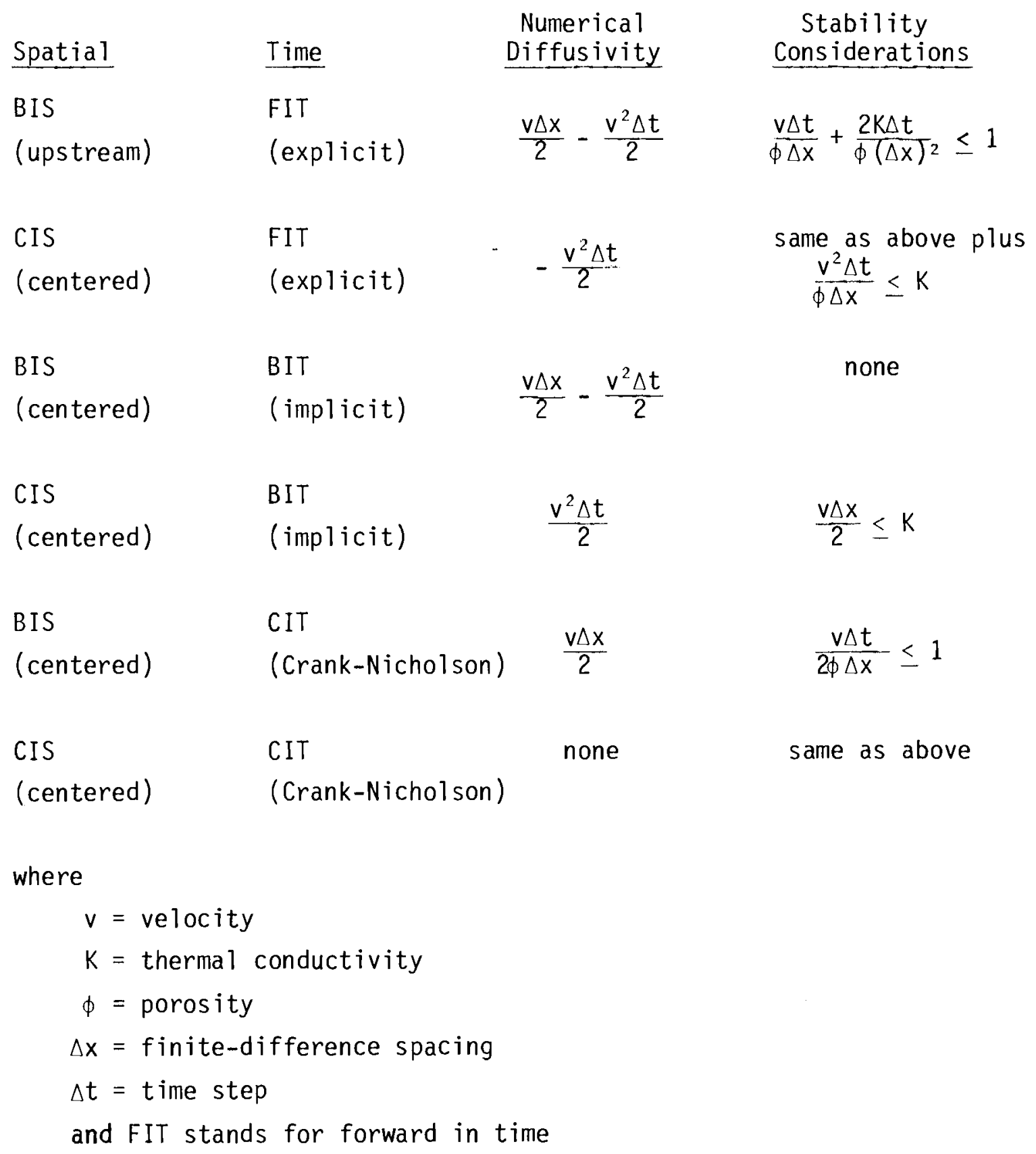


criteria can be derived for a radial system by substituting

$$
V=Q / 2 \pi r \Delta z
$$

and

$$
\Delta x=\Delta r=\frac{1}{r} \Delta \ln r .
$$

The finite-difference equations that result from the above approximations are solved sequentially (Coats et a1., 1974). Two solution techniques are provided in the model to solve the matrix equations. These are (1) a reduced bandwidth direction solution and (2) two line successive overrelaxation (L2SOR).

\section{Applications}

A summary of SWIP's options is presented in Table 8 . These options have been thoroughly tested by comparison to several analytical solutions including that of Avdonin (1964). The model has also been applied to numerous field problems including the first phase of the Auburn field experiment, which will be discussed later.

An advantage that SWIP offers is that overburden and underburden heat losses are computed without the need for including the confining layers in the grid, thus reducing the number of unknowns that are solved for in the matrix solution. The model also incorporates aquifer influence functions into the approximation. For example, the effects of an infinite or large aquifer may be included in the calculations without having to extend the finite-difference grid far away from the area of interest. This also reduces the number of unknowns, thus the simulation cost.

\section{GEOTHER}

\section{Governing Equations}

GEOTHER is one of several codes that were developed for geothermal applications, however, they are also applicable to ATES problems. Most of 
TABLE 8. Summary of Model Characteristics and Options for SWIP Code

Major assumptions:

- porous media

- single phase flow

Approximating method:

- finite-difference

Solution technique:

- reduced bandwidth direct

- L2SOR

Geometry:

- 1-, 2-, or 3-dimensional cartesian

- cylindrical

Options:

- steady or transient flow

- solute transport

- heat transport

- well bore

- heterogeneous and/or anisotropic media

- confined and/or water-table conditions

- recharge and/or wells

Boundary conditions:

- specified value

- specified flux

- aquifer influence functions 
the geothermal codes are similar in ability and only GEOTHER is discussed in this report. A discussion of other geothermal models may be found in Pinder (1979).

The final equations are formulated in terms of the dependent variables pressure and enthalpy, because these variables uniquely define the thermodynamic state of the system and because they are commonly obtained in a geothermal field situation. The equations are (Faust and Mercer 1979a)

$$
\frac{\partial(\phi \rho)}{\partial t}-\bar{\nabla} \cdot\left[\frac{k k_{r s} \rho_{s}}{\mu_{s}} \cdot\left(\bar{\nabla} \rho-\rho_{s} g \bar{\nabla} D\right)\right]-\bar{\nabla} \cdot\left[\frac{k k_{r w} \rho_{w}}{\mu_{w}} \cdot\left(\bar{\nabla} \rho-\rho_{w} g \bar{\nabla} D\right)\right]-q_{m}{ }^{\prime}=0
$$

and

$$
\begin{aligned}
\frac{\partial}{\partial t}[\phi \rho h & \left.+(1-\phi) \rho_{r} h_{r}\right]-\bar{\nabla} \cdot\left[\frac{k k_{r s} \rho_{s} h_{s}}{\mu_{s}} \cdot\left(\bar{v}_{\rho}-\rho_{s} g \bar{\nabla} D\right)\right] \\
& -\bar{\nabla}\left[\frac{k k_{r w} \rho^{h} w}{\mu_{w}} \cdot\left(\bar{\nabla} \rho-\rho_{w} g \bar{\nabla} D\right)\right]-\bar{\nabla} \cdot\left[K_{m}\left(\frac{\partial T}{\partial \rho}\right)_{h} \bar{\nabla}_{\rho}+k_{m}\left(\frac{\partial T}{\partial h}\right)_{p} \bar{\nabla} h\right] \\
& -q_{h}^{\prime}=0
\end{aligned}
$$

where the new terms are

$$
\begin{aligned}
h & =\text { specific enthalpy of the fluid ( } w=\text { water, s=steam), } \\
k_{r} & =\text { relative permeability ( } w=\text { water, s=steam), } \\
q^{\prime} & =\text { source term (m=mass, } h=\text { heat). }
\end{aligned}
$$

The enthalpy of the steam-water mixture is defined by

$$
h=\left(s_{s} \rho_{s} h_{s}+S_{w} \rho h_{w}\right) / \rho
$$

where $\rho$ is the density of the total steam-water mixture, defined as

$$
\rho=S_{w} \rho_{w}+S_{s} \rho_{S}
$$


and the volume saturations $\left(S_{S}\right.$ and $\left.S_{W}\right)$ are defined so that they sum to 1:

$$
S_{S}+S_{W}=1
$$

The final three-dimensional equations describe the two-phase flow of heat in a steam-water-rock system; however, with minor modification they also describe the flow of heat in a water-rock or a steam-rock system. When either steam or water is absent, the saturation of the absent phase is 0 and that for the existing phase is 1 . Further, it is assumed that the relative permeability of the absent phase is 0 and that for the existing phase is 1. Therefore, Equations (66) and (67) reduce to the appropriate equations for either the compressed-water region or the superheated-steam region. A solution for these equations will determine whether a specified location contains compressed water, a steam-water mixture, or superheated steam.

\section{Assumptions}

Assumptions discussed in Faust and Mercer (1979a) include:

(1) Capillary pressure effects are negligible.

(2) Thermal equilibrium exists among the steam, water, and rock.

(3) The reservoir fluid is single-component water consisting of either one or two phases.

(4) Relative permeability is a function of liquid volume saturation; hysteresis is neglected.

(5) Viscosities are considered functions of temperature.

(6) Porosity is a linear function of pressure, given by an equation similar to (16).

(7) Rock density, reservoir thickness, and intrinsic permeability are functions of space.

(8) Rock enthalpy is a linear function of temperature given by

$$
h_{r}=c_{r} T
$$

where $c_{r}$ is the specific heat of the rock and $T$ is temperature. 
(9) Darcy's equation for multi-phase flow is valid.

(10) Kinetic and potential energy, and the pressure material derivative, are negligible.

Solution

GEOTHER is based on a finite difference scheme using a block-centered grid. The grid allows variable spacing and can be used to approximate irregular reservoir geometry. The program is fully implicit, using NewtonRaphson iteration on nonlinear terms (Faust and Mercer 1979b).

For three-dimensional problems, the matrix equation is solved using SSOR (S1ice Successive Over-Relaxation). For one- and two-dimensional problems, the matrix solution technique is direct (Gauss-Doolittle decomposition).

\section{Applications}

A summary of GEOTHER's options is presented in Table 9. GEOTHER has been tested by comparison to analytical solutions, laboratory data and field data from Wairakei (Mercer and Faust 1979a). It was also used in a geothermal code comparison (Faust et al., 1980).

One of the analytical solutions used for testing was that given by Avdonin (1964). The results of this problem are presented in Figure 13. The original problem is described in Faust and Mercer (1979b); the results are shown to demonstrate the effects of midpoint and upstream weighting. It is clear that midpoint weighting approximates the temperature front better than upstream weighting but exhibits oscillations behind the front. Upstream weighting smears the front out by numerical diffusion (or dispersion) and does not exhibit oscillations (overshoot and undershoot).

As may be seen, GEOTHER may be used for single-phase problems. In addition, it may also be used for two-phase problems such as those discussed by Collins et al. (1978) where solar energy will be used to heat water to temperatures in excess of $600^{\circ} \mathrm{F}$, which will be injected and stored underground. 
TABLE 9. Summary of Model Characteristics and Options for GEOTHER Code

Major assumptions:

Approximating method:

Solution technique:

Geometry:

Options:

Boundary conditions:
- porous media

- single- and two-phase flow

- thermal equilibrium

- pure water

- finite-difference

- Newton-Raphson iteration

- slice successive over-relaxation

- Gauss-Doolittle decomposition

- 1-, 2-, or 3-dimensional cartesian

- cylindrical

- steady or transient flow

- heat transport

- two-phase flow

- heterogeneous and/or anisotropic media

- specified value

- specified flux 


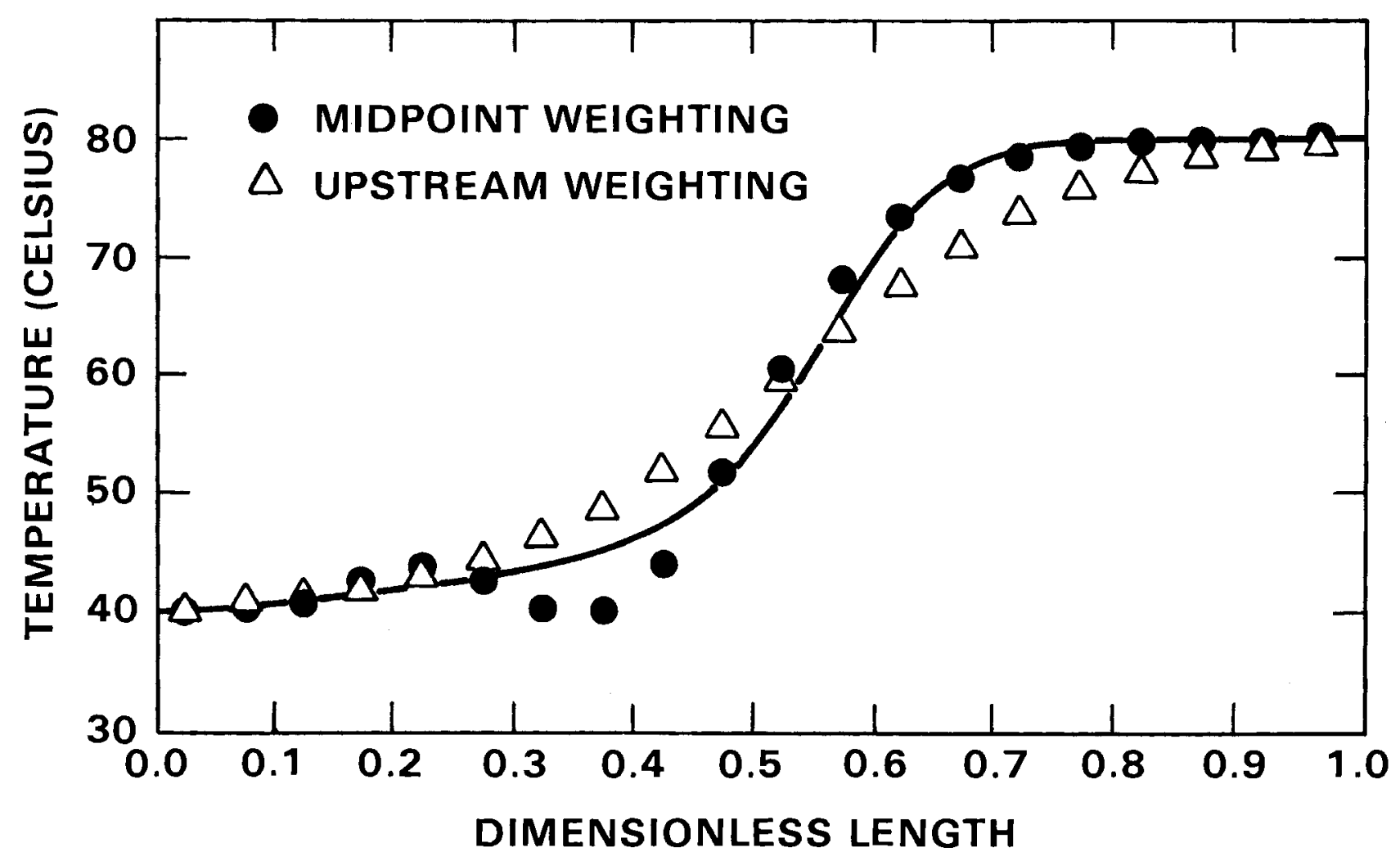

FIGURE 13. Avdonin analytic solution (solid line) for a time of $3.75 \times 10^{8} \mathrm{~s}$ and a reservoir length of $1187.5 \mathrm{~m}$ (distance measured from injection source is indicated as dimensionless). Also shown are finite-difference solutions for midpoint and upstream weighting (from Faust and Mercer 1979b). 


\section{TEXAS A\&M FINITE ELEMENT MODEL}

Governing Equations

The finite element model discussed in this section is presented in Grubaugh and Redde11 (1980). The equation for fluid flow is that given in Equation (18), whereas the heat transport equation is similar to Equation (36).

Assumptions

The following major assumptions were made in the derivation of the governing equations (Grubaugh and Reddell 1980):

(1) Both Darcy's Law and Fourier's Law are valid.

(2) Fluid density can be expanded about po, a reference density, in a Taylor series truncated with first order terms in temperature and pressure.

(3) Instantaneous thermal equilibrium is assumed between the fluid and solid phases at any point in the porous media.

\section{Solution}

The equations are solved using the finite element method, which is discussed in Appendix B. The matrix solution is direct. More specifically, a finite-difference approximation is used for the time derivative and a three-dimensional Galerkin finite element approximation for the space derivatives. According to Grubaugh and Reddell (1980), the use of a strict Galerkin approach led to unacceptable solution oscillations in sharp temperature front problems (i.e., problems where the temperature changes quickly over a small distance or time). Several techniques were tried in an attempt to correct the problem. Reduction of element and time step size proved ineffective in eliminating the sharp temperature front oscillation problem. An upstream weighting scheme corrected the oscillation problem, but resulted in an unacceptable smear of the sharp temperature front. A mass lumping scheme resulted in the best solution to sharp temperature front problems. The mass lumping scheme yielded solutions without the oscillation problem and with less smear than the upstream weighting scheme. 


\section{Applications}

A summary of the Texas A\&M code is presented in Table 10. The model has been applied to several analytical solutions including that by Avdonin (1964). It has also been applied to several hypothetical problems, but it has not been field tested. 


\section{TABLE 10. Summary of Model Characteristics}

and Options for the Texas A\&M Code

Major assumptions:

- porous media

- single-phase flow

- thermal equilibrium

Approximating method:

- finite element

Solution technique:

- direct

Geometry:

- 1-, 2-, and 3-dimensional cartesian

Options:

- steady or transient flow

- heat transport

- heterogeneous and/or anisotropic media

Boundary conditions:

- specified value

- specified flux 
GEOCHEMICAL MODELS

ATES requires an understanding of (1) the local or site-specific aquifer characteristics, (2) the economics of the heat producers and consumers, (3) the pumping and well bore equipment, and (4) chemical compatibilities between the injected liquid and the inplace liquid, the aquifer solid matrix, and any surrounding confining units. It has been shown that flow and transport models can be valuable tools for evaluating some of these aspects. In this section, we review some geochemical models presently available which can help evaluate chemical problems associated with ATES.

The geochemical models discussed in this section have a different purpose from the thermal and fluid flow models discussed earlier. The state of the geochemist's art has not yet reached the point where it is possible to calculate the reactions which will occur in any given mixture of waters or between any water and it's aquifer. The principles of thermodynamics enable one to calculate the tendency of reactions to occur as a system tends toward a state of equilibrium but data and a theoretical framework are not yet available to predict how fast these reactions will actually take place. The models described here are used to calculate the nature of chemical reactions which could occur for any given ATES scheme but they do not attempt to predict whether the reaction actually will occur and how fast. They are nonetheless very important to the evaluation of potential ATES schemes, for it is important to be certain that for example, the water to be injected will not have a tendency to precipitate an aquifer-clogging solid upon mixing with the formation water or to react with the formation itself so as to produce a solution capable of corroding the surface equipment when it is pumped back. In this section we review some geochemical models presently available which can help evaluate potential chemical problems of this type. Specifically, two types of models used by geochemists will be discussed. These types are here termed, aqueous solution models and geochemical reaction models. 
Aqueous solution models provide the methodology by which measured chemical compositions of solutions are transformed into such thermodynamically significant quantities as free ion activities. For example, the quantity important to determining whether a calcium-bearing mineral dissolves or precipitates is the thermodynamic activity of the free calcium ion in solution. What is measured in the chemical analysis of the solution is merely the concentration of the total calcium in solution. Models which permit the calculation of activities from concentrations are called aqueous solution or speciation models.

Geochemical reaction models describe the chemical reactions which result from, for example, mixing two solutions, addition of solids to a solution, and temperature changes to a solution or to a system of solids and a solution. These reactions are described in terms of the masses of elements which are transferred between solid phases and the solution or from one solid to another. Changes in solution chemistry from such a model are needed to determine the changes in solution density and viscosity which will affect the flow in the system. Changes in solid masses are related to changes in the volume in the system and have a potential to affect the aquifer porosity and permeability. Two types of geochemical reaction models are discussed.

A number of geochemical phenomena which have affected ATES programs, other water injection experiments, or low temperature natural geothermal systems are also discussed. Those included were selected as examples of problems which either were or could have been elucidated using existing geochemical models. 
Geochemical models are not a panacea, however, and some of their limitations are presented. Limitations resulting from assumptions made in their development and construction are considered, as are some practical limitations on their use.

\section{AQUEOUS SOLUTION MODELS}

This section contains a discussion of the conceptual models, as opposed to computer codes, which are available for describing the behavior of aqueous solutions. The discussion emphasizes the most commonly used conceptual model including its mathematical formulation for the solution of geochemical problems, how the equations are solved and embodied in several available computer codes and the general area of application of this model and the codes based on it.

\section{Conceptual Models}

The equations of classical thermodynamics apply in their most elegant and simple form only to ideal solutions. In order to apply them successfully to the description of real solutions, it is necessary to account for the non-ideal behavior of the solutions.

The thermodynamic properties of solutions have been investigated extensively and for many years. From these investigations, it is clear that while aqueous solutions are much too complicated to be described from principles using a quantum-statistical approach, it is still possible to adequately describe their behavior with equations containing relatively few semi-empirical parameters.

One of the most important parameters needed for aqueous solution modeling is the activity coefficient $(\gamma)$ which relates the thermodynamic activity (a) of a species with its mass $(\mathrm{m})$. The mass, or concentration of a solute is normally the measured parameter while the thermodynamic activity of the solute is the parameter which appears in the equations describing its thermodynamic behavior. The determination of the activity coefficient relating these two parameters is of the utmost importance to our ability to model behavior of an aqueous system. 
There are two major conceptual model types which are of use to geochemical modeling. These are the ion-association and the mixed electrolyte models. A summary of the conceptual basis for each of these follows.

\section{Ion-Association Model}

The conceptual solution model most often applied in geochemistry is the ion-association or ion-complexing model, originally due to Bjerrum. This model assumes that oppositely charged dissolved ions react to form complexes which can be treated as thermodynamic entities with the complex in equilibrium with the remaining free ions and the equilibrium described by a thermodynamic equilibrium constant. The popularity of this approach in geochemistry stems from its early use by Garrels and Thompson (1962) in developing a chemical model to explain the behavior of sea water. This model is further discussed in standard textbooks on aqueous geochemistry (Garrels and Christ 1965).

The ion-association model is in common usage by aqueous geochemists. The number of field studies successfully completed making use of it show that the model does in fact, validly represent the phenomena it is meant to portray when used within its limits. The recent review volume edited by Jenne (1979) contains a number of papers illustrating the successful application of this conceptual model to geochemical problems.

A point which has strongly favored the continued use of the ionassociation model is the existence of a suitable thermodynamic data base. The data base results from the general acceptance and use of the conceptual model to reduce many laboratory experiments on solution behavior. In spite of the wide availability of data, problems frequently occur which cannot be handled satisfactorily because data for some elements and complexes are not available. Shortccmings to modeling due to the lack of thermodynamic data are discussed in more detail in a later section. 
The most serious limitation to the applicability of complexing models is that they become less capable of representing real solution behavior as the salinity of the solution increases. In general, ion-association models behave well in solutions with salt contents as high as a few molal (moles/ $\mathrm{kg}$ of pure $\mathrm{H}_{2} \mathrm{O}$ ), certainly including the range of normal sea water which is 0.7 molal. At concentrations significantly greater and particularly in such highly saline waters as brines, the ion-association model breaks down and the mixed electrolyte conceptual model discussed next is preferred.

\section{Mixed-Electrolyte Model}

The mixed-electrolyte model for the behavior of solutions is a relatively recent theoretical development of Reilly et al. (1971) and Pitzer (1973) based on equations proposed by Guggenheim and Scatchard (see Lewis and Randal1 1961).

The mixed-electrolyte theory does not assume the existence of explicitly defined complex ions in solution but instead writes an expression for the activity coefficient of individual ions as functions of the concentrations of all ions in solution. This way it considers the contributions of other ions to the activity of the ion of interest but does so in a lumped parameter way rather than as discreetly defined individual complex ions.

The advantage of this conceptual approach is that it is able to reproduce the properties of solutions of all concentrations including those far more saline than could be satisfactorily treated using the ion-association mode 1. This has been demonstrated in recent work by Eugster et a 1 . (1980) who use a mixed-electrolyte model to simulate the sequence of minerals which deposit from evaporating sea water. Extremely good experimental data are available on this mineral sequence and by successfully simulating it, Eugster et al. can be considered to have validated the use of mixed-electrolyte models for geochemical problems.

Such models, successful as they might be, cannot presently be applied to a wide range of geochemical problems because of their severly restricted data base. At the present time, for example, data are available only for systems containing the elements $\mathrm{Na}-\mathrm{K}-\mathrm{Mg}-\mathrm{Ca}-\mathrm{SO}_{4}-\mathrm{Cl}-\mathrm{H}_{2} \mathrm{O}$. It is probable that carbonate ions will be added to this list in the near future which 
will make simulation of many ground waters feasible. The mixed-electrolyte will still have little application in ATES work though because to date, no data are available at other than $25^{\circ} \mathrm{C}$.

The mixed-electrolyte model has been mentioned here for the sake of completeness and because it will probably form the basis of many future geochemical modeling schemes. At the present time though, its applications are so limited that it will not be discussed further.

Ion-Association Models

Governing Equations

Two sets of equations can be written and solved to determine the distribution of species in an aqueous solution.

A mass balance equation can be written for each element in the solution as

$$
m_{T, \underline{i}}=m_{\underline{i}}+\sum_{\underline{j}=1}^{J} n_{\underline{i j}} m_{\underline{j}}, \quad \underline{i}=1,2, \ldots, I
$$

where $m_{\underline{i}}=$ concentration of free $i$ of of species $\underline{i}$;

$$
\begin{aligned}
& m_{\underline{j}}=\text { concentration of complex } i \text { on } \underline{j} \text { containing } n_{j \underline{j}} \text { moles of } \underline{i} \text {; } \\
& m_{T, \underline{i}}=\text { total concentration of } \underline{i} \text {, often the analyzed concentration } \\
& \text { input to the model. }
\end{aligned}
$$

Likewise, a mass action equation is available for each association reaction forming a complex ion

$$
a_{j}=k_{j} \prod_{\underline{i}=1}^{I} a_{\underline{i}}^{n_{i j}}, \quad \underline{j}=1,2, \ldots, J
$$

$$
\text { where } \begin{aligned}
a_{\underline{j}} & =\text { activity of complex ion } \underline{j} ; \\
a_{\underline{i}} & =\text { activity of free ions, } \underline{i} ; \\
n_{\underline{i j}} & =\text { stoichiometric coefficient of ion } \underline{i} \text { in species } \underline{j} ;
\end{aligned}
$$




$$
\begin{aligned}
& \mathrm{K}_{\mathrm{j}}=\text { equilibrium constant for formation of complex } \underline{j} \text {; and } \\
& \pi \text { indicates a product over } i=1 \text { to } I \text {. }
\end{aligned}
$$

Generally, analytical values are available for the total concentrations of each element, giving I values of $m_{T}$. Also, equilibrium constants are known for each complex ion considered in the model, giving $J$ values of $\mathrm{K}$. Values of al1 the stoichiometric coefficients $n_{\underline{i j}}$ are also known. By relating the free and complex ion concentrations, $m$, and activities, $a$, the set of Equations (72) and (73) are solvable.

The activity coefficient, $\gamma$, relates activities and concentrations in the equation

$$
a_{i}=\gamma_{\underline{i}} m_{i}
$$

Activity coefficients are evaluated experimentally using the departures from ideal behavior observed in such properties of real solutions as osmotic pressures, vapor pressures, electrochemical potentials, and diffusion rates. These measurements show that activity coefficients vary from ion to ion and with temperature and total ion concentration in the solution.

The Debye-Hückel theory of ion behavior in solution reproduces measured activity coefficients in dilute solutions. The Debye-Hückel theory itself is described by Lewis and Randal1 (1961) and its application to geochemical problems by Garrels and Christ (1965) and by Helgeson (1969). The DebyeHückel equation can be written

$$
\log \gamma=-\frac{A z^{2} \sqrt{I}}{1+B a \sqrt{I}}+b I
$$

where $A$ and $B$ are known functions of the dielectric constant, density and temperature of the solvent, water;

$z=$ ionic charge;

$a=$ ion size parameter;

$b=$ ion specific parameter dependent on the temperature and the solvent. 


$$
\begin{aligned}
I & =\text { ionic strength of the solution, } \\
& =1 / 2 \sum_{\underline{k}} m_{\underline{k}} z^{2}, \quad k=1,2, \ldots k \\
& \text { for all } \underline{k}(=\underline{I}+\underline{J}) \text { dissolved ions and complexes. }
\end{aligned}
$$

The first term on the right of Equation (75) is the original Debye-Hückel expression which is able to reproduce measured activity coefficients in solutions with ionic strengths no higher than 0.05 to 0.1 molal. Inclusion of the second term extends the range of applicability to soluticns of ionic strengths of several molal. As pointed out in the previous section, though, in more concentrated solutions the quite different mixed-electrolyte approach to solution behavior and activity coefficient formulation is required. Truesdell and Jones (1974) discuss activity coefficient calculation and provide values for use in Equation (75).

As an example of the application of the general Equations (72), (73), and (74), consider a solution containing calcium and sulfate. The major ion association reactions will be

$$
\mathrm{Ca}^{+2}+\mathrm{SO}_{4}^{-2}=\mathrm{CaSO}_{4}^{\mathrm{O}}
$$

The mass balance equation for calcium is written

$$
\mathrm{m}_{\mathrm{T}, \mathrm{Ca}}+2=\mathrm{Ca}_{\mathrm{Ca}}+2+\mathrm{maSO}_{4}^{0}
$$

and that for sulfate:

$$
\mathrm{m}_{\mathrm{T}, \mathrm{SO}_{4}^{-2}}=\mathrm{m}_{\mathrm{SO}_{4}^{-2}}+\mathrm{m}^{\mathrm{CaSO}_{4}^{\circ}}
$$


The mass action equation corresponding to the complex-formation reaction (76) is written

$$
{ }^{\mathrm{a}} \mathrm{CaSO}_{4}^{\circ}=\mathrm{K}_{\mathrm{CaSO}_{4}^{\circ}}{ }^{\mathrm{a}} \mathrm{Ca}^{+2} \quad{ }^{\mathrm{a}} \mathrm{SO}_{4}^{-2}
$$

Using activity coefficients and Equation (74) allows this equation to be written in terms of concentrations as

$$
\mathrm{m}_{\mathrm{CaSO}_{4}}=\frac{{ }_{\mathrm{CaSO}}{ }_{4}{ }^{\mathrm{m}}{ }_{\mathrm{Ca}}+2{ }^{\gamma}{ }_{\mathrm{Ca}}+2{ }^{\mathrm{m}} \mathrm{SO}_{4}^{-2}{ }^{\gamma} \mathrm{SO}_{4}^{-2}}{{ }_{\mathrm{CaSO}_{4}}^{\circ}}
$$

A phenomenon not accounted for in the general Equations (72) and (73) is that the solvent itself may be involved in certain of the complex forming reactions. The solvent, water, is involved in any reaction in which hydroxide or hydrogen ions participate. The activities of $\mathrm{H}^{+}$and $\mathrm{OH}^{-}$are connected by association expression for water

$$
\mathrm{H}^{+}+\mathrm{OH}^{-}=\mathrm{H}_{2} \mathrm{O}
$$

but there still exists one more unknown than equation.

Two approaches are taken to account for this additional unknown. Frequently, the measured $\mathrm{H}^{+}$activity $\left(=10^{-\mathrm{pH}}\right)$ is specified as input, along with total concentrations of other dissolved elements. The other approach makes use of the fact that a solution can have no net electrical change. This electro-neutrality principle is embodied in the equation

$$
0=\sum_{\underline{k}} \underline{m}_{\underline{k}} z_{\underline{k}} \text { over all } \underline{k}(=I+J) \text { dissolved ions. }
$$

If this equation is included in the set to be solved, the hydrogen ion activity can be calculated, rather than required as input data. 
Given these sets of equations, and input data on concentrations, equilibrium constants and subsidiary constant values for Equation (75), for example, it is possible to calculate the activities and concentrations of all dissolved ions and ion complexes in a given solution. The equations to be solved are highly nonlinear, though, so in the next section, techniques for their solution representative of those presently used in computer models are discussed.

\section{Solution Techniques}

All chemical equilibrium calculations whether they be in gases or liquids are based on the solution of sets of mass balance and mass action equations similar to (72) and (73) of the preceding section. The nonlinearity of this set of equations has caused chemists to consider for many years how they might best be solved. Van Zeggeren and Storey (1970) discuss a number of the approaches which have been taken for the calculation of chemical equilibrium. Much of their discussion centers on work done on gas phase reaction modeling but many of the techniques are applicable as well to aqueous modeling.

A number of computer models for the calculation of solution equilibrium are available at the present time. In all of these models only three basic solution types are used. These are simple iterative methods, Newton-Raphson iteration, and solution by means of monotone sequences. Solution equilibrium programs are described in a review paper by Nordstrom et a1. (1979). These authors describe the solution techniques used by each of the individual programs as well as providing the primary references for detailed descriptions of the programs themselves. Potter (1979) has also reviewed certain of these and other present geochemical models but in considerably less detail than Nordstrom et al. A partial list of the models discussed in this section is presented in Table 11 . Note the temperature and pressure ranges over which the various models are valid. Also indicated are the number of gases and aqueous species considered by each model.

A number of the most commonly used aqueous speciation programs use simple iterative back substitution methods to solve the combined sets of mass balance and mass action equations. The first generation programs 
Table 11. Partial Summary of Geochemical Mode1s

\begin{tabular}{|c|c|c|c|c|c|}
\hline Reference & Mode1 & Aqueous Sp & Gases & $\begin{array}{l}\text { Temperature } \\
\text { Range } \mathrm{C} \\
\end{array}$ & $\begin{array}{c}\text { Pressure } \\
\text { Range-Bars }\end{array}$ \\
\hline $\begin{array}{l}\text { Kharaka \& } \\
\text { Barnes (1973) }\end{array}$ & SOLMNEQ & 181 & 3 & $0-300$ & $1-1000$ \\
\hline $\begin{array}{l}\text { Plummer } \\
\text { et al. (1979) }\end{array}$ & WATEQF & 105 & 3 & $0-100$ & 1 \\
\hline $\begin{array}{l}\text { Ball et a1. } \\
\text { (1979) }\end{array}$ & WATEQ2 & 220 & 3 & $0-100$ & 1 \\
\hline $\begin{array}{l}\text { Nordstrom } \\
\text { et a1. (1979) }\end{array}$ & GEOCHEM & 2000 & 2 & 25 & 1 \\
\hline $\begin{array}{l}\text { Nordstrom } \\
\text { et a1. (1979) }\end{array}$ & $\begin{array}{l}\text { MINEQL/ } \\
\text { REDEQL2 }\end{array}$ & * & * & 25 & 1 \\
\hline Wolery (1979) & EQ3 & 140 & 8 & $0-300$ & $\begin{array}{l}\text { to steam } \\
\text { saturation } \\
\text { or } 500 \text { bars }\end{array}$ \\
\hline \multicolumn{6}{|c|}{ * depends on data base } \\
\hline
\end{tabular}


SOLMNEQ (Kharaka and Barnes 1973) and WATEQ (Truesdel1 and Jones 1974) use a straightforward approach in which the mass action expressions are substituted directly into the mass balance equations. Wigley (9177) has shown that an algebraic rearrangement of the equations into a continued fraction form leads to convergence with significantly fewer iterations.

These methods can be demonstrated and contrasted using the aqueous solution described by Equations (77), (78), and (79). Assuming for simplicity that all activity coefficients equal one, allows the direct substitution of the mass action expression (79) into the mass balance Equations (77) and (78):

$$
\begin{aligned}
& \mathrm{m}_{\mathrm{T}, \mathrm{Ca}}+2=\mathrm{m}_{\mathrm{Ca}}+2+\mathrm{K}_{\mathrm{CaSO}} \mathrm{O}_{4} \mathrm{~m}_{\mathrm{Ca}}+2 \mathrm{~m}_{4}^{-2} \\
& \mathrm{~m}_{\mathrm{T}, \mathrm{SO}_{4}^{-2}}=\mathrm{m}_{\mathrm{SO}_{4}^{-2}}+\mathrm{K}_{\mathrm{CaSO}_{4}}{ }_{\mathrm{Ca}} \mathrm{m}_{\mathrm{Ca}}+\mathrm{m}_{4}^{-2}
\end{aligned}
$$

In the direct back substitution scheme, these equations are rearranged and programmed as

$$
\begin{aligned}
& { }_{\mathrm{Ca}}{ }^{+2}, \mathrm{n}+1=\mathrm{m}_{\mathrm{T}, \mathrm{Ca}}+2-\mathrm{KaSO}_{4}^{\circ}{ }_{\mathrm{Ca}}{ }^{+2}, \mathrm{n}{ }_{\mathrm{SO}_{4}^{-2}, \mathrm{n}}^{\mathrm{m}} \\
& \mathrm{m}_{4}^{-2}, \mathrm{n}+1=\mathrm{m}_{\mathrm{T}, \mathrm{SO}_{4}^{-2}}{ }^{-\mathrm{K}} \mathrm{CaSO}_{4}^{\circ}{ }^{\mathrm{m}} \mathrm{Ca}^{+2}, \mathrm{n} \quad \mathrm{SO}_{4}^{-2}, \mathrm{n}
\end{aligned}
$$

For the first iteration, $(n=1)$, the free ion concentrations are set equal to their measured total concentrations. Iteration continues until the concentration changes per iteration fall below some preset value.

The continued fraction approach rearranges and programs Equations (83) and (84) slightly differently:

$$
{ }_{\mathrm{Ca}}^{\mathrm{m}}+\mathrm{n}+1=\frac{\mathrm{m}}{\mathrm{T}, \mathrm{Ca}}+2
$$




$$
\mathrm{sO}_{4}^{-2}, \mathrm{n}+1=\frac{{ }^{\mathrm{T}}, \mathrm{SO}_{4}^{-2}}{1+\mathrm{K} \mathrm{CaSO}_{4}^{\circ}{ }^{\mathrm{m} \mathrm{Ca}^{+2}, \mathrm{n}}}
$$

For the first iteration, the free ion concentrations are again set equal to the measured total concentrations. Because of its relatively rapid rate of convergence, the continued fraction method has been adopted for such second generation programs as WATEQF (P1ummer et a1. 1976) and WATEQ2 (Ba11 et al. 1979).

A Newton-Raphson approach was adopted for the solution of the mass balance and mass action equations in another one of the early aqueous equilibrium programs, MINEQL (Morel and Morgan 1972). Although apparently successful, the technique has not been widely adopted in other aqueous speciation models although it does appear in programs such as REDEQL 2 and GEOCHEM (described by Nordstrom et a1. 1979). As described in a later section, however, NewtonRaphson techniques are commonly used in a solution of geochemical reaction problems.

The third method for solving this set of nonlinear equations is the use of a monotone sequence (Wolery and Walters 1975). This method, which is in essence an extension of a continued fraction method, offers an increased stability with respect to a wide range of aqueous solution compositions used as input. This method is used only in the program EQ3 (Wolery 1979).

- In addition to the nonlinearity resulting from the need to combine additive mass balance with multiplicative mass action equations, a much less strong nonlinearity exists in ion-association models. During the course of the iterative solutions of whatever type, the modeled solution composition changes. This leads to changes in ionic strength and in the values of the activity coefficients (Eq. 75), which appear in the actual concentration equations solved. These changes are accounted for by determining new activity coefficients based on ionic strength values calculated for the distribution of species existing at the start of each iteration. Activity coefficients vary only slowly with ionic strengths in solutions to which the ion-association model is applicable. Thus, their changes at each iteration are too small to prevent or delay convergence. 


\section{Applications}

Aqueous speciation models have two main purposes. The first is to provide a thermodynamically adequate description of a solution given analyzed element total concentration data. The second is to use this description of the solution to determine the affinities of various reactions which the solution could undergo. Most programs of this type do not actually calculate affinities but rather a similar quantity called the saturation index (SI).

The thermodynamic description of the solution comprises simply the activities calculated from the solution of the mass balance and mass action equations, and the concentrations derived from them. The values of these solution species activities are of importance, less for their own sake, than because they form the basis of subsequent calculations, the results of which are of practical importance. For example, solution activities must be known at the start of and during the progress of the chemical reaction calculations described in the next section.

The results of most aqueous speciation programs are used to calculate reaction quotients for a number of minerals with which the solution could potentially react. Reaction quotients $(Q)$, sometimes called ion activity products (IAP), are identical in form to the solution equilibrium constants (KT) of minerals. That is

$$
Q_{\underline{j}}=I_{\underline{j}}=\prod_{\underline{i}} a_{\underline{i}}^{n} \underline{j}
$$

where the species activities, $a_{i}$, correspond to the actual solution being examined and the product is over all i. On the other hand, the solution equilibrium constant at temperature $T$ is

$$
K T_{\underline{j}}=\underset{\underline{i}}{\pi} a_{\underline{i} \underline{i j}}^{n} e q
$$

where the species activities, $a_{i}$, eq, are those obtained when the solution is in equilibrium with the mineral $j$. 
The tendency for a mineral, $\underline{j}$, to dissolve in or precipitate from a solution is indicated by comparing the IAP and KT using the saturation index:

$$
S I_{\underline{j}}=\log \frac{\text { IAP }_{j}}{K T_{\underline{j}}}
$$

If $S I=0$, the mineral and solution are at equilibrium; if $S I>0$, the solution will tend to precipitate the mineral, while if $S I<0$, the mineral will tend to dissolve. The ability to predict the tendency for a given water to dissolve or precipitate minerals is one of the most important contributions chemical modeling can make to ATES project design.

The affinity, A, of a reaction is another important result of aqueous speciation modeling. It is written as

$$
\underline{A}_{\underline{j}}=R T \ln \left(\frac{{ }^{K T}}{\underline{j}}{ }_{\underline{\underline{j}}}^{Q_{\underline{j}}}\right)
$$

where $R$ is the gas constant and $T$ the absolute temperature (Prigogine and Defay 1954). Like the saturation index, the affinity indicates the potential for reaction (aithough with opposite sign) so that when $\underline{A}_{\underline{j}}>0$, the mineral $\underline{j}$ will tend to dissolve; when $\underline{A}_{-j}<0$, it will tend to precipitate from the solution, and when $\underline{A}_{\underline{j}}=0$, the mineral and solution are at equilibrium.

The significance of the affinity is that for reactions not too far removed from equilbrium, it is a measure of reaction rate. The conduit from equilibrium thermodynamics, as represented by reaction affinity, to reaction kinetics, represented by rates, is Eyring's absolute reaction rate theory and, particularly, the expression:

$$
r_{\underline{j}}=k_{\underline{j}}\left(1-\exp \left(\underline{-A_{j}} / R T\right)\right)
$$


where $r_{j}$ is the overall rate of reaction $\underline{j}, k_{j}$ is a constant characteristic of $\underline{j}$ and the other terms are as before. Aagaard (1979) provides a recent evaluation of reaction rates in geochemistry, and the subject is mentioned again in the next section.

\section{GEOCHEMICAL REACTION MODELS}

The models described in the previous section were static in the sense that they provide only a description of the state of the system under the conditions of, for example, temperature and total elemental concentrations, input to them. Saturation indices are indicators of the tendency for reactions to occur but the aqueous speciation models are not capable of predicting what changes in either solution composition or in composition and mass of associated solids might occur as a result of these reactions.

The models discussed in this section are specifically designed to describe the changes in solutions composition and in relative masses of solids which may occur as a solution reacts toward equilibrium with solids, gases or other fluids with which it is brought into contact. The reaction description produced by these models is in terms of the masses of material which move from phase to phase in the course of the reaction. Thus, they are known variously as mass transfer or as reaction path models.

Two approaches toward reaction calculations have been used to develop the presently available codes. In the first of these, mass transfer modeling, the code solves directly for the final equilibrium state of the system from the initial conditions specified for it. In the reaction path approach on the other hand, small steps in reaction progress are taken from the initial specified state toward equilibrium so that the entire reaction path from initial to final state is worked out rather than simply the mass transfer involved in the overall reaction from initial to final states. These two model types are discussed in more detail in the following two sections.

\section{Mass Transfer Models}

Mass transfer models are similar to the aqueous speciation models in that they assume that a state of equilibrium extends throughout the entire 
system being examined by them. That is, they assume that not only do the aqueous phase reactions occur rapidly, but also that all potential solid phase solution or precipitation reactions occur equally rapidly.

\section{Governing Equations}

Solution reactions with solid or gas phases are handled in much the same way as are ion complexation reactions in the solution itself. For each mineral or gas reacting, a mass action expression can be written similar to that of Equation (73):

$$
k_{p}=\prod_{i} a_{i}^{n} n_{i p}, \quad p=1,2, \ldots, \quad p
$$

where

$$
\begin{aligned}
a_{\underline{i}} & =\text { activities of dissolved free ions } \underline{i} ; \\
k_{p} & =\text { equilibrium constant for the dissolution of phase } \underline{p} ; \\
n_{\underline{i p}} & =\text { stoichiometric coefficient of ion } \underline{i} \text { in phase } \underline{p} .
\end{aligned}
$$

The addition of this equation to the set of equations describing the solution allows the addition of another unknown to the mass balance equations for each ion $i$. This additional term is simply the number of moles transferred between the solution and the phase $p$.

For example, consider that the solution described earlier was brought in contact with a mineral of composition $\mathrm{CaSO}_{4}$ and that the amount of mass transferred between the mineral and solution phases in bringing the solidliquid system to equilibrium was sought. At equilibrium, the mass action expression for the solution (79) will continue to hold and in addition, the dissolution equilibrium constant for the solid (equivalent to Equation 90) must also hold.

$$
\mathrm{KaSO}_{4}=\underset{\mathrm{Ca}}{\mathrm{Ca}} \stackrel{\mathrm{a}}{\mathrm{SO}_{4}^{-2}}
$$

The addition of this second mass action equation permits the inclusion of an 
additional unknown in the mass balance Equations (77) and (78) as follows:

$$
\begin{aligned}
& \mathrm{m}_{\mathrm{T}, \mathrm{Ca}}+2=\mathrm{m}_{\mathrm{Ca}}+2+\mathrm{m}_{\mathrm{CaSO}_{4}}+{ }^{\Delta \mathrm{m}_{\mathrm{CaSO}_{4}}} \\
& \mathrm{~m}_{\mathrm{T}, \mathrm{SO}_{4}^{-2}}=\mathrm{m}_{\mathrm{SO}_{4}^{-2}}+\mathrm{m}_{\mathrm{CaSO}_{4}^{\circ}}+\Delta \mathrm{m}_{\mathrm{CaSO}_{4}}
\end{aligned}
$$

This additional term, $\Delta \mathrm{m}_{\mathrm{CaSO}}{ }_{4}$, is simply the amount of mass transfer required between the solid and the liquid phases to bring this system to equilibrium from its initial state.

Solution Techniques

The group of equations which must be solved by mass transfer codes of this type are essentially identical with those solved by ion-association models. Thus, it is not surprising that essentially the same solution techniques have been applied to this type of mass transfer model as were described previously for ion-association models.

A recent and rather efficient code of this type is PHREEQE (Parkhurst et al. 1980). This code uses a continued fraction formulation and simple iteration to solve its mass balance equations while it used a Newton-Raphson scheme to solve the remaining equations.

Another code which has limited mass transfer capabilities is MINEQL and its descendant REDEQL 2 (described by Nordstrom et al. 1979). These codes use Newton-Raphson iteration throughout.

\section{Applications}

Direct mass transfer calculating codes are of particular utility for several reasons. First, by virtue of their structure, they tend to run rapidly and efficiently and therefore can be used at little cost. They are valuable for performing calculations in systems in which rapid reactions are likely to occur. Problems to which they are particularly suitable include solution mixing problems and solution reaction with minerals that are likely to dissolve or precipitate rapidly. 
Some types of minerals, particularly silicates, react slowly with solutions or may react incongruently--that is, in such a way that one of their reaction products is another solid which tends to shield further reaction with the first solid. Under these conditions of slow approach to equilibrium, the direct mass transfer calculating codes are inappropriate and reaction path codes based on a description of incremental or differential mass transfer must be used.

Reaction Path Models

In reaction path modeling, it is assumed that equilibrium exists in the solution phase and between the solution and the reaction products. That is, during the course of progress of a reaction, equilibrium will continue to hold among dissolved species, and further, if the solution becomes oversaturated with respect to a phase, that phase will immediately form in whatever quantity is required to return the solution to equilibrium. The particular value of this approach to the study of geologic processes is that, unlike the mass transfer models discussed previously, it does not require the entire system to be at equilibrium. Thus, it permits the simulation of the path by which a system originally at dis-equilibrium reacts toward equilibrium. Information from this path simulation includes the masses of solid phases dissolved or formed during each reaction step rather than simply the overall mass transfer required to go from the initial to the final equilibrium state.

\section{Governing Equations}

Reaction path models are based on the principles of irreversible or non-equilibrium thermodynamics. The development of the equations by which these principles are applied to geochemical problems is due to Helgeson (1968, 1969, 1979). Computer codes which permit the application of these equations to real problems are provided in the work by Helgeson and his students (Helgeson et a1. 1970) and the results of applications of these codes by Helgeson and colleagues (Helgeson 1979; Helgeson et al. 1969).

Basically, Helgeson's approach consists of differentiating equations describing the reaction to be tracked with respect to a progress variable $\xi$. 
These progress variables, which may be different for each of the reactions considered, can be thought of as the relative rates of the reactions. The series of differential equations, together with the mass action and mass balance equations necessary to describe the equilibrium within the fluid phase, are sufficient to define the problem and in principle, can be solved for the amount of mass transferred at any stage of reaction progress.

\section{Solution Techniques}

The original computer code embodying Helgeson's approach was called PATHI or PATHCALC (Helgeson et al 1970). In this code the differential equations describing mass transfer were solved by finite-difference technique. An aqueous speciation model first produced a description of the initial solution and calculated the affinities with respect to possible products of reaction between the solution and existing specified phases (see Equation 92). Then small arbitrary steps in reaction progress were taken in the direction of reducing affinities. Following each step of reaction progress the new solution was re-solved and affinities calculated again. When phase boundaries were reached (when the affinity of any reaction became zero or negative) a process of interval-halving was used to more precisely define the exact value of the reaction progress variable at equilibrium between the solution and that phase.

Obviously such a solution procedure is very slow to compute and furthermore, has a tendency to drift. So, after many steps of reaction progress, such a model tends to produce system compositions different from those which would result from a one-step mass transfer calculation.

In order to reduce the computer time required for simulations using PATHI, high-order predictor-corrector methods of integration were put into a version of the program developed at Los Alamos Scientific Laboratory (Herrick, written communication 1976, reported by wolery 1979). Programs of this type, called FASTPATH, are presently in use by several investigators. This modification improves the running speed of the program markedly and it diminishes, but does not eliminate, the problems of drift. 
Another approach to the solution of reaction path computations has been taken by wolery (1979) in EQ6. This program is a combination of the direct mass transfer approach of the previous section with the differential pathfollowing approach of PATHI. While reaction paths continue to be calculated by approximations to Helgeson's differential equations, the compositions at points where reaction affinities reach zero are calculated directly by applying a mass transfer approach like that described in the previous section. Any drift introduced into the problem during the approach to the equilibrium position is corrected out by this direct solution for equilibrium mass transfer.

\section{Applications}

Even the most recently developed reaction path programs such as EQ6, execute considerably more slowly than the direct mass transfer programs. Generally, the geochemical understanding of the system will be such that its definition using mass transfer computations will suffice. However, there will occasionally arise a problem in which the identities and amounts of intermediate reaction products may be important and for these problems it is necessary to use reaction path models of the sort described here.

\section{MODEL APPLICATIONS}

The models which have been discussed are capable of predicting the occurrence of a number of chemical phenomena which may be important to ATES. In this section a number of these phenomena are discussed along with examples taken from the geochemical literature of the use of models for their elucidation. Where possible, the examples have been taken from the ATES or geothermal literature.

\section{Effects of Changing Temperature/Pressure}

The equilibrium positions of all chemical reactions will be influenced by changes in temperature and in pressure. The size of the temperature effect depends on the enthalpy of the reaction, because, the equilibrium positions of highly endothermic or exothernic reactions will change more extensively with temperature than will those reactions with very small enthalpies of reaction. 
The pressure effects on a reaction are dependent on the volume change accompanying that reaction. The equilibrium position of a reaction, which is accompanied by a large positive or negative volume change, will be much more strongly influenced by a pressure change than the small volume change.

Gas Solubility

Volume changes which accompany reactions in the liquid and solid phases are likely to be relatively small under the conditions which will be encountered in ATES projects. Thus, pressure effects on the equilibrium position on solid and liquid reactions are likely to be minimal and can be neglected in most chemical modeling schemes. Reactions in which gases are evolved or dissolved are accompanied by large volume changes and, thus, pressure effects must be explicitly taken into account. Available geochemical models tend not to include pressure effects even on gases because they are generally used on systems in which no separate gas phase is present. This assumption is made because ground water tends to be sufficiently confined to prevent the formation of a separate gas phase.

The development of a gas phase would be something to guard against in any ATES scheme because of the detrimental effect its presence would have on the permeability of a porous medium. To guard against this eventuality, some experimental injection projects have included degassifiers in their plant to insure that the water delivered to the injection well was as gas free as possible (Koch et al. 1973; Ragone and Vecchioli 1975). It is not known whether calculations based on the known chemistry on the water to be injected and the injection system properties were made in order to justify the inclusion of the degassifier. The test reports suggest that degassifier operation actually had little effect on the injectability of the system (Vecchioli and $\mathrm{Ku}$ 1972). Temperature effects are also important to gas solubilities and most geochemical models include routines for calculating gas partial pressures at various temperatures using simple Henry's Law formulations. The higher pressures which exist at depth within geothernal systems are frequently sufficient to hold in solution gas which evolves when the geothermal fluid is brought to the surface and the pressure on it is lowered. 
Geochemical models have been used to calculate from surface measured properties what the down-hole conditions were before gas evolved from the fluid. Truesdell and Singers (1974) describe a model which permits the calculation of aquifer chemistry given gas volume and water chemical information measured at the surface. Their computer code uses an aqueous model similar to that of WATEQ and was designed and used to study the chemistry of the Wairakei genthermal system. Pearson et al. (1978) have used model calculations to provide concordance among $\mathrm{pH}$, alkalinity and carbon dioxide partial pressure values measured at a number of low temperature geothermal springs. In several of these systems, the out-gasing which resulted from the pressure drop as the water approached the surface led to precipitation of carbonate minerals and therefore, affected the near-surface flow systems associated with the springs.

\section{Mineral Solubility}

The solubilities of such solids as aquifer minerals or surface plant construction material will in general be functions of temperature. Solids must be expected to dissolve in or precipitate from fluids associated with ATES. This increase or decrease in solid matter may result in increases or decreases in aquifer porosity and permeability or in scale build-up on, or corrosive solution of surface pumps, piping, and wellbore construction material. These phenomena will generally have unfortunate effects on the performance of an ATES plant and their potential occurrence shouid be investigated beforehand as much as possible using geochemical models.

The effects of temperature on the position of chemical equilibria are explicitly included in all of the generally available models discussed earlier. This is done by including in them, expressions which determine the values of the equilibrium constants in the mass action Equations (73) and (94) as functions of temperature. The temperature also effects the values of the activity coefficients $\gamma$ needed to interconnect the mass action and the mass balance sets of equations. The effects of small temperature changes can be estimated by using the aqueous speciation models and noting how the saturation indices in Equation (91) vary with temperature. A more rigorous approach to simulating temperature change effects is to use mass 
transfer or reaction path models to calculate the actual amounts of material dissolved or precipitated as a result of a temperature change. Amounts so calculated are much more useful in estimating whether the properties of the surface plant or the aquifer are likely to be seriously degraded by the operation of an ATES system.

The effects of temperature changes on high temperature geothermal fluids are well known and have often been reviewed. Arnфsson (1981) for example, has discussed the problem of mineral deposition as it affects the utilization of Icelandic geothermal waters. Less work has been done on mineral precipitation or solution effects in lower temperature geothermal waters. Bedinger et a1. (1979) and Pearson et a1. (1978) have used model calculations to show that a combination of carbon dioxide gas evolution and temperature change results in the precipitation of calcite in the Arkansas Hot Springs at about $60^{\circ} \mathrm{C}$.

One of the more commonly troublesome materials in geothermal systems is silica $\left(\mathrm{SiO}_{2}\right)$, the solubility of which is strongly a function of temperature. Geothermal waters exceeding perhaps $100^{\circ} \mathrm{C}$ are commonly in equilibrium with one or another silica mineral. Some waters with temperatures as $10 \mathrm{w}$ as $60^{\circ} \mathrm{C}$ (Bedinger et a1. 1979) or lower (Hobba et al. 1979) also appear to be close to equilibrium with silica. The precipitation of silica from cooling waters tends to be sluggish at temperatures be $10 \mathrm{w} 100^{\circ} \mathrm{C}$ and thus, it is one of the least satisfactory materials on which to apply an equilibrium-based model of the types discussed above. Silica is also one of the few materials on which the results of careful kinetic experimental work are available (Rimstidt and Barnes 1980). Thus, it may be possible to include this kinetic information in suitable reaction path models in order to predict the effect of silica precipitation and dissolution.

Despois and Nougarede (1979) have reported the possibility of permeability reductions due to silica dissolution. They explain that if the silica dissolves from the solid matrix into the liquid phase, the grains in the solid matrix become smaller and uncemented. Thus, the possibility for compaction is enhanced, which may reduce the aquifer permeability. 
Effects of Mixing Injected and Formation Waters

It is a fundamental precept of the ongoing ATES work that injected and receiving fluids be from a single aquifer. Injection and recovery wells will penetrate the same formations, and hence a temperature difference will exist between the injected and native fluids. However, if the fluid injected as part of an ATES scheme is different in chemical composition as well as in temperature from the native fluid present in the aquifer, chemical incompatibilities may exist. It is well known that mixing two fluids often produces a mixture capable of dissolving or precipitating material that neither of the end member fluids would tend to react with. An example would be the mixing in a beaker or by injection of a sulfate-bearing fluid with one containing a high strontium content. The resulting solution could be supersaturated with respect to strontium sulfate and would therefore tend to precipitate that substance. The end member solutions, though, could have low enough strontium and sulfate contents respectively, to be unsaturated with strontium sulfate.

There are also more subtle effects of mixing which can result in mineral precipitation or solution. These have been shown to occur in natural mixed water systems and so could equally well occur in an ATES system. The geochemical models used to develop an understanding of natural mixed systems are the types described above. Description of some of this work serves to illustrate how such models could be applied to potential ATES mixing phenomena.

A classical geochemical mixing problem is that of predicting the effects of mixing a calcite $\left(\mathrm{CaCO}_{3}\right)$ saturated fresh ground water with normal ocean water, which is also calcite saturated but of a quite different ionic strength. Such mixing occurs naturally at the periphery of such limestone peninsulas as Florida and the Yucatan where calcite saturated ground water discharges to the ocean. Because of the great difference in ionic strengths between the fresh and ocean waters, the activity coefficients of the dissolved species in the mixtures will vary nonlinearly between those of the end members. The appication of a modified aqueous speciation model to this problem was described by Plunmer (1975). 
The application of a more sophisticated mass transfer model to a field mixing problem is described by Back et al. (1979). These authors compared modeled and measured water compositions resulting from the mixing of fresh carbonate ground water with ocean water along the east coast of the Yucatan Peninsula. They conclude that while the ability to dissolve calcite is greater in the mixtures, the mixing also causes $\mathrm{CO}_{2}$ gas evolution which limits the ability of the mixtures actually to dissolve calcite. Such gas evolution would be less likely to occur under the confined conditions of an injection site, but this study serves as an illustration of the application of models to mixing problems.

In several other studies, models applied to actual recharge experiments required that mixing of waters be considered. The dominant geochemical effect in these studies was not the mixing phenomena, though, but the effect of the injected water on the formation itself. Therefore, these studies are mentioned in the next section.

\section{Effect of Injected Water On Formation Mineral Solubilities}

Ground waters tend to be at chemical equilibrium with respect to the reactive minerals in the aquifer. It has been demonstrated in many groundwater geochemical studies that, for example, waters in limestones tend to be in equilibrium with calcite $\left(\mathrm{CaCO}_{3}\right)$ and, if their residence time is long enough, with dolomite $\left(\mathrm{CaMg}\left(\mathrm{CO}_{3}\right)_{2}\right)$ as well. Likewise, waters associated with gypsum $\left(\mathrm{CaSO}_{4}, 2 \mathrm{H}_{2} \mathrm{O}\right)$ or halite $(\mathrm{NaCl})$ bearing rocks tend to be saturated with these minerals as well. At normal temperatures, ground waters are normally not at equilibrium with respect to silicate minerals which may be present in their aquifers because of the slow rate of reaction of such minerals. In geothermal systems though, where reaction rates are more rapid, the waters are frequently saturated with respect at least to one of the common silica $\left(\mathrm{SiO}_{2}\right)$ phases such as quartz or chalcedony. No strict lower limit can be placed on the temperature at which a water is likely to be silica saturated but at least one system is known--the Arkansas Hot Springs, with waters saturated with respect to chalcedony at about $60^{\circ} \mathrm{C}$ (Bedinger et al. 1979). 
An injected fluid will in general be different chemically from the fluid it displaces. It is therefore not likely to be at equilibrium with respect to the reactive minerals in the aquifer and may well react with them. These reactions could increase or decrease the aquifer porosity; hence, permeability, depending on whether they tend to dissolve or precipitate minerals. They could also result in changes to the water cremistry which will degrade its utility once it is pumped back to the surface. Such changes might include an increase in iron content which could cause rusting or fouling problems in the surface equipment or an increase in silica or carbonate content which could cause scaling. They might also increase the dissolved gas content of the water so that during the pressure drop associated with withdrawal pumping, a gas phase could form in the screen, pump, or surface lines.

Geochemical models can in principle be used to describe the reactions resulting from an initial dis-equilibrium between the injected fluid and the host aquifer. A mass transfer model together with a knowledge of the injected water chemistry, the aquifer mineralogy and native water chemistry could be used to calculate the mass dissolved or precipitated as various mixtures of injected and native water react with the aquifer material toward equilibrium. This information would show quantitatively the amount of mineral mass likely to be formed or destroyed by these reactions which could then be used to assess the amount of damage to the aquifer which might result from them. Likewise, the resulting chemistry of the fluid which would later be withdrawn could be predicted and estimates made of any harmful effects its chemistry might have on the surface plant.

Mass transfer modeling of the type described in the previous paragraph has not yet been done on an injection scheme. However, there are several examples of the use of aqueous solution models and the saturation indices calculated from them to shed light on reactions which occurred in several experimental injection schemes. Such models permit a choice between several hypothetical reaction paths and permit manual mass balance calculations to 
be performed. These models, however, did not perform the mass transfer calculations necessary for a complete quantitative understanding of the behavior of the system.

For many years, the petroleum industry has been injecting fluids of one type or another into their reservoirs to enhance petroleum recovery. Many of these injection schemes had operational difficulties because of reactions between waters and between injected water and formation minerals. Subcasky (1978) has recently reviewed petroleum industry experiences as they might apply to ATES problems. The industry does not seem to rely heavily on the use of models to predict potentially deleterious effects of injection schemes. Instead it relies on laboratory and bench tests in which the actual waters and aquifer material involved are mixed and the results observed before the actual injection takes place.

Two well-known injection research experiments reported in the literature have chemical data suitable for model use in their interpretation. These are a tertiary-treated sewage injection experiment carried out at Bay Park, Long Island, New York (Vecchioli and Ku 1972; Koch et al. 1973; Vecchioli et al. 1974; and Ragone 1977) and an experiment in which fresh water was artificially recharged into a brackish water aquifer at Norfolk, Virginia (Brown and Silvey 1977).

In the Norfolk experiment, degradation of the injection specific capacity was noted as was a turbidity when the injected water was withdrawn from the formation. A cause of both turbidity and the clogging was determined to be the dispersion of clay minerals present in the aquifer. These minerals were flocculated in the presence of the brackish formation water but became dispersed when that water was displaced by the fresher injected water. In the dispersed state, these clays moved and clogged aquifer pores degrading the ability of the aquifer to accept water. Also, while dispersed, they appeared in the withdrawn water as turbidity. Chemical analys is of the water withdrawn from the well after the injection showed that a change in 
the calcium to sodium ratio of the injected water had taken place during its residence in the formation. Calcium originally present in the injected water was replaced by sodium from the aquifer by a reaction which could be shown to be a clay cation exchange reaction. Calcium clays are considerably less likely to disperse than are sodium clays even in the presence of fresh water, therefore, it was possible to prevent additional dispersion of formation clays in later tests by introducing a strong calcium chloride solution at the start of subsequent fresh water injection cycles.

Available chemical models do not predict whether a clay present in the formation is likely to disperse or not with a given change in salinity but they might well have predicted the sodium for calcium exchange which would tend to discourage dispersion. Thus, a state of the art geochemical model applied at the beginning of such an injection scheme might have suggested that the calcium chloride solution should be injected. Had this been done early enough, it might have prevented the permanent deterioration of the aquifer which occurred as a result of first injecting fresh water alone.

In the Bay Park experiment, the aquifer was considerably less reactive than was the Norfolk aquifer, although a number of exchange reactions similar to those noted in the Norfolk experiment also occurred. The injection specific capacity of the well at Bay Park decreased with injection. This was due less to chemical changes within the aquifer than it was to physical clogging in the screen and overpack due to turbidity in the injected water and to bacterial growth on the screen.

One potentially deleterious change in the water chemistry did occur at Bay Park as a result of injection water reaction with aquifer materials. Both the native formation water and the injected water had very low dissolved iron contents. As field data and chemical modeling showed, this was because the formation water in the Bay Park area is reducing and its iron content is controlled by the solubility of the sulfide mineral pyrite $\left(\mathrm{FeS}_{2}\right)$. The injected water on the other hand, tended to be oxidizing and its iron content 
was probably set by a sparingly soluble ferric hydroxide mineral $\left(\mathrm{Fe}(\mathrm{OH})_{3}\right)$. Where the formation waters mixed a range of chemistries existed in which both iron minerals are relatively soluble. Thus, waters in this mixed zone had analyzed iron contents of several milligrams per liter rather than the levels of less than a few hundredths of a milligram per liter found in both the formation and the injected water. While this phenomena was not a problen in the Bay Park experiment, it could be in a longer experiment. For example, iron transported through the formation in such a mixing front could precipitate and degrade the aquifer. Iron so brought into solution could also have a detrimental effect on the surface works of an ATES application. In the Bay Park experiment aqueous solution models were used to elucidate the reaction described above in order to explain the observed high iron concentrations in the mixing zone. No attempt was made to calculate the actual amounts of iron by using more sophisticated mass transfer models. In an actual ATES application, the application of both aqueous solution and mass transfer models before the injection itself takes place would permit such effects to be predicted.

MODEL LIMITATIONS

This discussion on geochertical modeling would not be complete without a section on some of the limitations on geochemical modeling in ATES programs. These 1 imitations can be treated in two broad categories. The first are those which result from inherent weaknesses in the assumptions made to construct the models. To overcome these limitations will require a new generation of model types. The second category of limitations simply restricts the range of problems to which existing models can be applied. Limitations In Model Assumptions

The fundamental assumption underlying the formulation of all the models previously discussed, is that equilibrium exists throughout some part of the system, and that it can be described by classical chemical thermodynamic equations. Thus, except in the description of dissolution reactions in the 
reaction path models, the effects of variable reaction rates cannot be included in the present generation of geochemical models. From the standpoint of the chemistry of the solution itself, the equilibrium assumption can probably always be justified but equilibrium between solids and solution may not always be a valid assumption. In applying geochemical models it is necessary always to explicitly consider whether the fundamental equilibrium assumption is appropriate for the system being examined.

The generally available models described above simulate only the chemical aspects of a system's behavior. As several experimental injection programs have shown, though, physical and biological effects may also affect injection systems. Some of the geochemical results of injection experiments at Bay Park, New York were rientioned. While there were several interesting and potentially predictable geochemical effects which accompanied this injection, the experiment work at the facility showed that the actual clogging of the system was biological and physical rather than chemical. In some of the tests, decreases in injection specific capacity could be correlated with turbidity in the injected water (Ragone et al. 1975). In other tests, with less turbid injection water, specific capacity decreases were also noted. These were attributed to the growth on the screen and in the gravel pack of bacteria and other slime producing organisms (Vecchioli 1970; Vecchioli and $\mathrm{Ku}$ 1972). Geochemical models do not include either of these types of phenomena.

Injection tests made at Norfolk, Virginia were also described and it was pointed out that cation exchange reactions on the clays in the system could in principle at least have been predicted using geochemical models. The deterioration of aquifer permeability noted, though, was due to dispersion of the clays. While this dispersion can be thought of as a chemical phenonemon in that it results from a decreasing ionic strength of the water as a result of injection, it is still not the type of chemical phenomena included in the present generation of geochemical models. Thus, the models would not have been adequate to predict its occurrence. 
Limitations To Model Use

The limitations discussed in this section are practical limitations and restrict the types of problems to which the models can be applied. They are to be contrasted with the theoretical limitations discussed in the previous section.

The differences between ion-complexing and electrolyte types of conceptual solution models were described. While all generally available codes embody the ion-complexing model, this model is limited to solutions with ionic strengths less than a few molal. The state-of-the-art of geochemical modeling does not preclude the application of ATES to brine systems. However, for completeness, it is necessary to reiterate here that presently available geochemical models are limited practically to less saline waters.

The use of geochemical models can also be severely limited by the availability of thermodynamic data. While reasonably good data exist for important solid and solution species of a number of common elements, there are still certain data deficiencies which may be important. For example, cation exchange in clay minerals is frequently a very important control on the chemistry of ground waters, yet little data are available to permit its modeling (Thorstenson et al. 1979).

It is also possible that in a given ATES application, some less common element, strontium for example, or iron or manganese, may be potentially troublesome. While much thermodynamic data do exist for less common elements, the thermodynamic data base may still not be extensive enough to provide assurance that the system can in fact be adequately predicted.

Absence of other data also limits the use of geochemical models. According to Stottlemyre et al. (1979), prior to field development, the following geochemical and related data should be collected for an ATES site: 
- Ground water

cation and anion concentrations

dissolved silica

$\mathrm{pH}$

Eh

suspended solids

dissolved gases

- Rock or sediment

particulate plugging information

water sensitivity

mineralogy

bulk chemistry

thermo-mechanical properties

thermal diffusivity

Suspended solid, particulate plugging information, water sensitivity, thermochemical properties, and thermal diffusivity are not explicitly needed for geochemical modeling. However, the remaining detailed data are essential but rarely available. Thus the use of geochemical models is restricted. 


\section{APPLICATIONS OF TRANSPORT MODELS}

\section{REVIEW OF FIELD APPLICATIONS}

In addition to physical and chemical processes, mathematical descriptions, and the solution techniques presented, it is necessary to discuss actual examples and site-specific factors associated with ATES. Detailed descriptions of the mathematical techniques and the physio-chemical processes, though interesting theoretically, are of little value unless they are related to typical applications and natural conditions.

This discussion of site-specific considerations begins with a review of completed and on-going field applications. This will serve as a backdrop for the specific considerations associated with alternative aquifer characteristics, rock types, and chemical and fluid characteristics. The relevance of particular characteristics to the choice of appropriate analytical and numerical techniques for evaluation of ATES systems is also addressed.

An attempt is made to review the field studies in chronological order. Emphasis is placed on data and modeling results, if any. Those that have been completed are summarized in Table 12, which is extended from that given in Tsang et al. (1980a). Also, three on-going demonstration field studies are discussed. These demonstration projects are located at Minneapolis-St. Paul, Minnesota, Stony Brook, New York, and Bethel, Alaska. All of these studies, except the Minnesota project, have utilized or will utilize unconsolidated sediments as storage mediums. The Minnesota project will utilize a cemented sandstone sequence; the geologic conditions of the Alaska project have not been reported. The more detailed case histories are presented according to (1) location, (2) hydrogeology, (3) description of field operations, and (4) general discussion.

Besides the projects to be discussed in detail, a number of other field projects have been or are currently being conducted in a variety of countries, for which little or no data are available. For example, Sauty et al. (1978) mentioned two 1974 injection/production projects without interim storage at 
TABLE 12. Data From ATES Field Experiments

(extended and modified from Tsang, et a 1., 1980a)

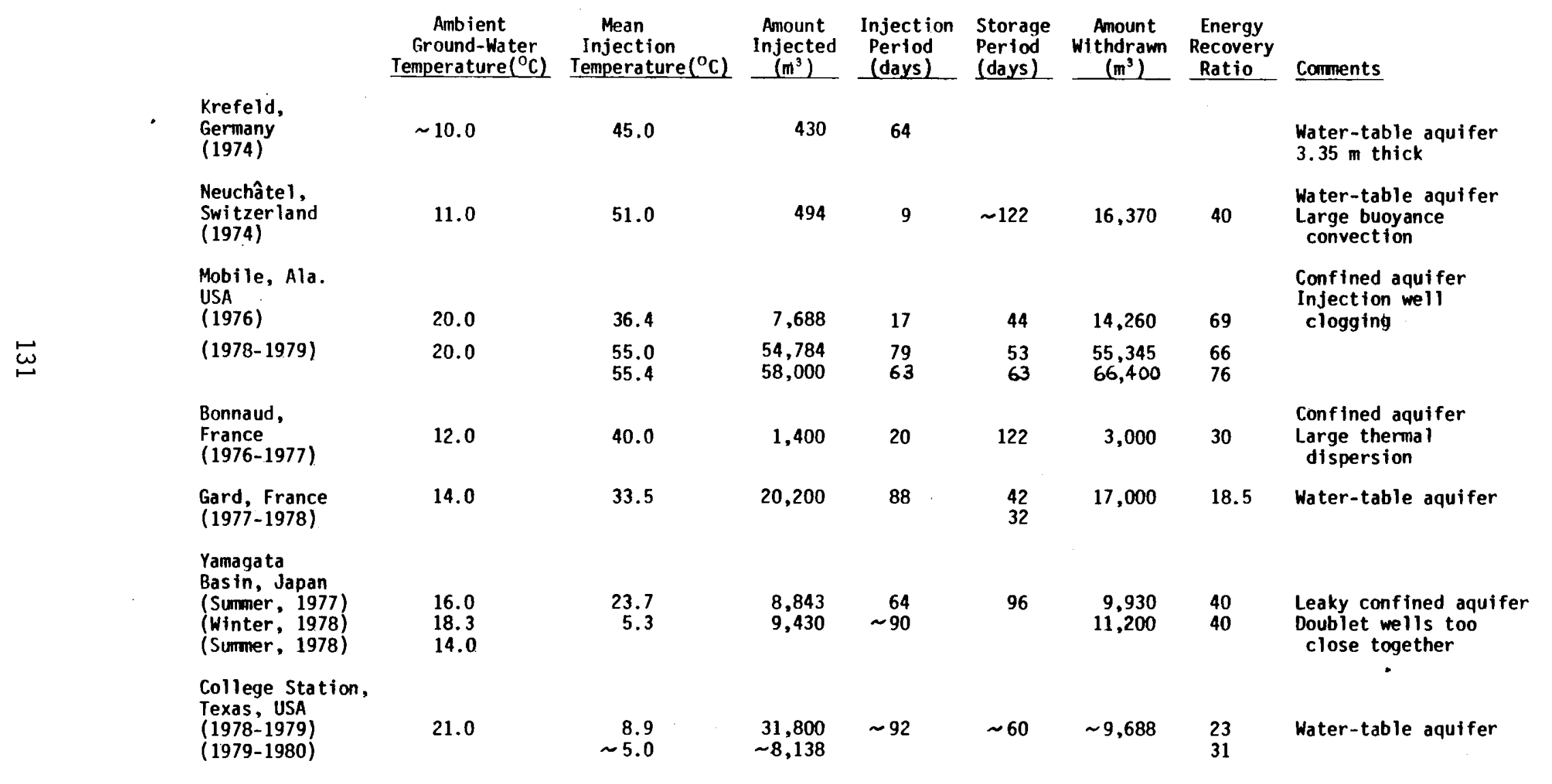


Neuilly and Noisy, France. Also, the Lawrence Berkeley Laboratory ATES Newsletters have mentioned studies in Germany and China. The German field tests were performed at Münsterlander Bucht, West Germany in 1978 and 1979 (ATES Newsletter, September 1979) and additional pilot studies were planned to utilize an aquifer in the vicinity of the Munich-Grosshadern Hospital in late 1980 or 1981 (ATES Newsletter, June 1980). Chinese projects occurred at Shanghai and Beijing. They entailed producing cool aquifer water for industrial use and re-injecting the spent warm water after treatment. These projects were primarily meant to combat land subsidence (ATES Newsletter, May 1979 and June 1980). Finally, Voss (1980) and Voss et al. (1981) have reported an ATES project in Stidsvig, Sweden. This project entailed the storage of industrial waste heat in an esker in southern Sweden and the use of mathematical models to evaluate the aquifer hydraulics and the subsequent heat transport analysis.

Krefeld, Germany

\section{Location}

This ATES site is located near Krefeld, Germany. The study was performed under the auspices of the Geological Survey of North RhineWestphalia. The test to be described began on December 27, 1973 and ended on March 1, 1974 (Werner and Kley 1977).

Hydrogeology

The storage aquifer was a shallow unconsolidated phreatic aquifer $3 \mathrm{~m}$ thick. It overlies a $6.75 \mathrm{~m}$ clay unit which confines a deeper sand and gravel aquifer. The effective porosity of the unconfined aquifer was $23 \%$ and its natural flow velocity was determined as $1 \mathrm{~cm} /$ day (Werner and Kley 1977).

\section{Description of Field Operations}

This test was considered preliminary to future long-term tests (Meyer et a1. 1976). It was an injection test only. The procedure entailed injecting $430 \mathrm{~m}^{3}$ of water at $45^{\circ} \mathrm{C}$ at a flow rate of $0.3 \mathrm{~m}^{3} / \mathrm{hr}$ for 64 days (Werner and Kley 1977). Temperature measurements were made from which 
heat profiles were constructed. Pre-injection temperatures were not reported. Also, a finite-difference model was developed to simulate the experimental results (Werner and Kley 1977 ).

\section{Discussion}

The aim of this study was to ascertain a quantitative physical basis for the analysis of ATES technical problems through experimentation and mathematical modeling. An experiment was performed and a finite-difference model was used to simulate the experimental results. The model was axisymmetric and it was based on a two-dimensional convective-diffusive heat transport equation. It simulated a three-layered system. As shown in Figure 14, good agreement between numerical and experimental results was obtained (Werner and Kley 1977).

It was noted that about one-third of the energy injected was lost to the top, base, and surrounding aquifer material through heat conduction; and that there was no evidence of horizontal thermal stratification (Meyer et al. 1976). The heat losses were most likely due to the unconfined condition of the aquifer, the slow injection rate, the small volume of injected hot water, and the use of steel casings in the injection and observation wells. The lack of thermal stratification was most likely due to the thinness of the aquifer.

Overal1, Werner and Kley (1977) emphasize that the success of an ATES project depends on the proposed heat storage scheme and on the local conditions. Local conditions include aquifer proximity, aquifer physical properties, the proximity of the energy producers and consumers, and any governing water laws. The actual heat storage scheme involves the managerial decisions of storage volumes, single or well doublet systems, and injection/ production cycles. These conditions can be evaluated without large scale experimentation through numerical modeling and managerial decision making. 
INJECTION WELL

BF
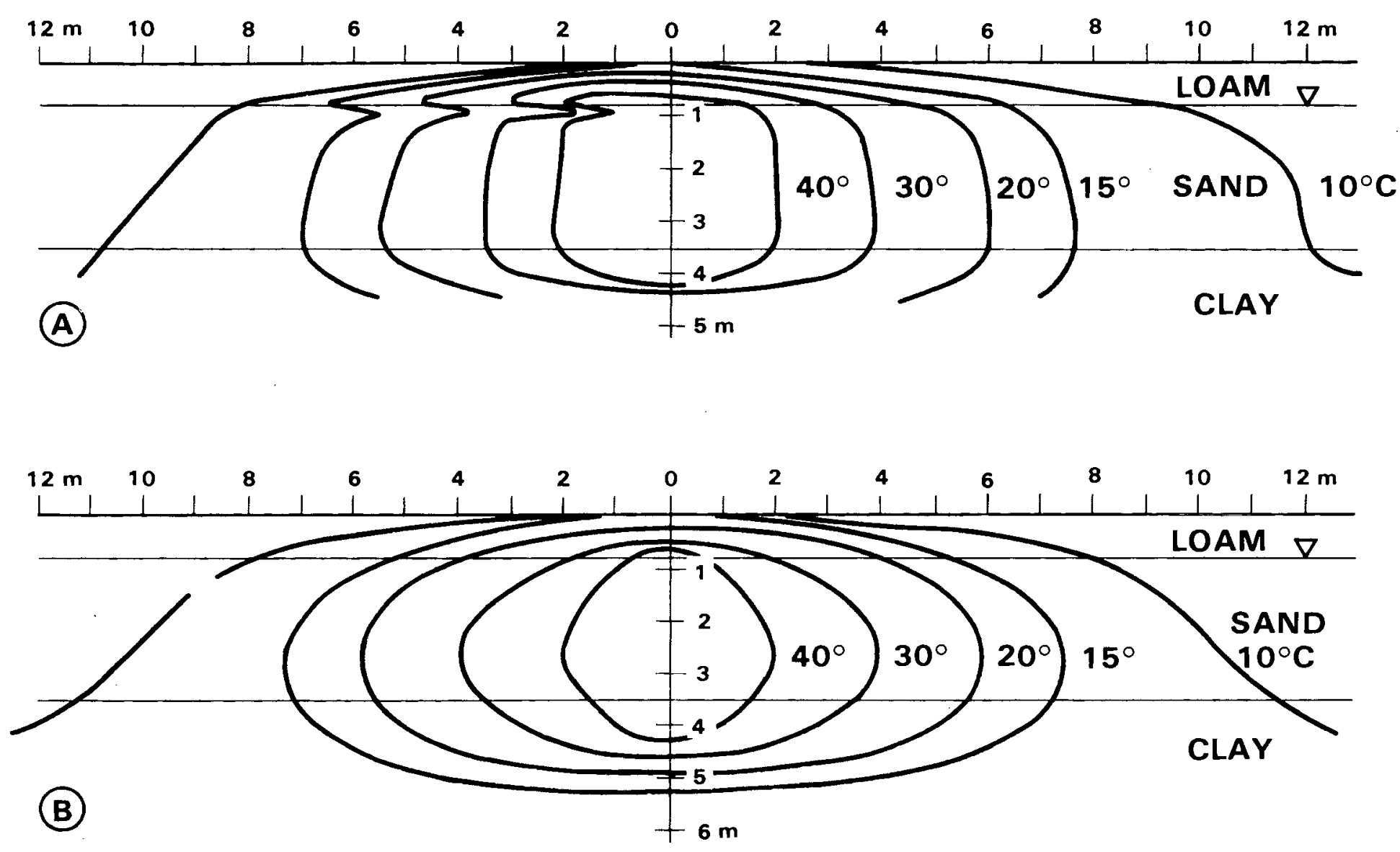

FIGURE 14. Isotherm Illustration in Longitudinal Section Parallel to the Flow of the Ground Water A. Field Experiment; B. Calculation Mode 1 (from Werner and Kley 1977) 
Neuchâtel, Switzerland

\section{Location}

This ATES site is located on the Areuse delta near Lake Neuchâtel, Neuchâtel, Switzerland. The study was financed by the Fonds National Suisse pour la Recherche Scientifique and it was performed under the auspices of three agencies: (1) Laboratoire de Géotechnique de l'École Polytechnique Féderale, Laussanne, (2) Laboratoire de Production d'Énergie de l'École Polytechnique Féderale, Laussanne, and (3) Centre d'Hydrogéologie de l'Université de Neuchâtel, Neuchâtel (Mathey 1977). The test to be described was performed in 1974 (Tsang et al. 1980a).

\section{Hydrogeology}

The storage formation used in this study is a phreatic aquifer known as the Columbier-Robinson aquifer. This aquifer consists of three sand and gravel layers, totalling $10 \mathrm{~m}$ thick, which overlie an argillaceous loam with chalk. The hydraulic properties of the aquifer have not been reported in detail. The only property reported was hydraulic conductivity (K), which was $1.0^{\circ} \times 10^{-2}, 3.0 \times 10^{-3}$, and $6.0 \times 10^{-3} \mathrm{~m} / \mathrm{s}$ for the upper, middle, and lower layers of the aquifer, respectively (Mathey, 1977). The ground-water levels, under natural conditions, are controlled by the stage of Lake Neuchâtel such that the ground water flows into the lake under a potential gradient on the order of $10^{-4}$. The depth to water was not given (Mathey 1977; Tsang et a1. 1980a).

\section{Description of Field Operations}

The test reported is the fifth of a water pumping or injection series. It consisted of three stages:

(1) 223 hours (about 9 days) of injecting $494 \mathrm{~m}^{3}$ of water at $51^{\circ} \mathrm{C}$;

(2) a 4 month storage period; and

(3) 28 days of water withdrawal totalling $16,370 \mathrm{~m}^{3}$ of water. The original ground-water temperature was $11^{\circ} \mathrm{C}$. During the experiment, 720 thermal profiles were collected from twelve piezometers. The energy recovery ratio for the total withdrawn volume of water was $40 \%$; when the withdrawn volume equaled the injection volume, the energy recovery ratio 
was only 7\% (Mathey 1977). The evaluation of experimental results was aided by the use of a mathematical model which was calibrated to simulate the extension and behavior of the thermal disturbance within the aquifer.

\section{Discussion}

The purpose of this study was to observe and evaluate the development, displacement, and resorption of a thermal disturbance within a natural aquifer. This purpose was accomplished via experimentation, monitoring, and mathematical analysis. However, there were some difficulties.

The energy recovery ratio was not satisfactory considering the large production volume required to achieve $40 \%$ recovery. According to Tsang et al. (1980a), this was due to the high permeability of the aquifer, the lack of an overlying confining bed, and the sriall injection volume. The high permeability made possible the strong thermal stratification which led to heat losses through the unsaturated zone. On the other hand, Mathey (1977) concluded insignificant heat losses but important thermal dilution of the stored heat due to thermal conduction. He suggests that thermal losses were balanced by heat contributions during the summer.

Difficulties were also encountered in the mathematical analysis. The mathematical model used a numerical integration scheme to solve the convective-diffusive equation for heat transport in radial coordinates. Complete details concerning the model were not reported in Mathey (1977), Tsang (1979), or Tsang et a1. (1980a). However, Mathey (1977) did note that the mode1 was axisymmetric and that it calculated thermal balances for both the saturated and unsaturated zones of the aquifer. The difficulty arose in simulating the natural convection or bouyancy flow in terms of radial coordinates. Mathey (1977) pointed out that the use of numerical methods to forecast the extension of a thermal disturbance in a radial system proved troublesome. He attributed this to the lack of a theoretical basis in describing natural convection in a radial system.

In addition, another experiment is currently in progress at this same site. The objectives of the new experiment are: 
(1) to test a thermal disturbance in the ground water,

(2) to test for thermal leakage in the unsaturated zone, and

(3) to make physio-chemical and bacteriological analysis of the water (ATES Newsletter, September 1980).

Mobile, Al abama

Location

The experiment was conducted at a site near Mobile, Alabama. It was conducted in two phases by personnel from Auburn University. The first phase took place in 1976 and consisted of one cycle of injection, storage, and recovery. This work is described in Molz et al. (1978) and the associated simulation is presented in Papadopulos and Larson (1978).

The second phase consisted of two injection-storage-recovery cycles. This phase occurred in 1978-1979 and is described in Molz et al. (1980) and Molz et al. (1979). The associated model for this phase is described in Tsang et al. (1980b).

\section{Hydrogeology}

A relatively uniform confined aquifer, approximately $21 \mathrm{~m}$ thick, was used for the experiment. The aquifer consists primarily of a medium to fine sand with interspersed clay and silt comprising about $15 \%$ by weight. It occurs from approximately $40 \mathrm{~m}$ to $61 \mathrm{~m}$ below land surface and is overlain by a clay sequence about $9 \mathrm{~m}$ thick. The aquifer is bounded at its base by another clay sequence of undetermined thickness. The lateral extent of the aquifer is not known.

An analysis of time-drawdown data from an observation well showed departures from the theoretical type curve for infinite confined aquifers. These indicate the presence of impermeable or lowly-permeable boundaries. The transmissivity and storage coefficient were determined using early-time data (Papadopulos and Larson 1978) to be $940 \mathrm{~m}^{2} / \mathrm{d}$ and $5 \times 10^{-4}$, respectively. Using the theory of images, distances to the boundaries were determined to be about $360 \mathrm{~m}$ and $1200 \mathrm{~m}$ from the observation well. This data was also analyzed by Doughty et al. (1979), who obtained similar results. 


\section{Description of Field Operations}

During the first phase of the Mobile study, water at a temperature of $36^{\circ} \mathrm{C}$ was injected. The exact amount of water injected into the aquifer is uncertain because of a possible breach of the confining layers. It was estimated, however, that approximately $7,700 \mathrm{~m}^{3}$ were stored for a 36 day period. Withdrawal indicated a recovery factor of 0.69 .

For the second phase, the first six-month injection-storage-production cycle involved the storage of $55,000 \mathrm{~m}^{3}$ of water at about $55^{\circ} \mathrm{C}$. The injection took 79 days, whereas the storage period was 53 days. Production consisted of pumping at an average rate of $1,350 \mathrm{~m}^{3} / \mathrm{d}$ until the recovered water temperature fell to about $33^{\circ} \mathrm{C}$. At that point, $66 \%$ of the injected energy was recovered. The second injection-storage-production cycle was performed in a similar fashion using $58,000 \mathrm{~m}^{3}$ of water at an average temperature of $55.4^{\circ} \mathrm{C}$. This time when the production temperature had decreased to $33^{\circ} \mathrm{C}$, a recovery of $76 \%$ of the injected energy was accomplished.

\section{Discussion}

The first phase of this study was simulated by Papadopulos and Larson (1978) using the code SWIP (Intercomp 1976). Because of the aquifer boundaries (whose actual location was not known), difficulties during injection, and a low natural hydraulic gradient, the conceptual model for the experiment was simplified to consist of a cylindrical portion of the aquifer extending to a radial distance of $57.3 \mathrm{~m}$. This radius corresponds to the average radial distance to several observation wells where pressure data were available. The average of the water levels in these wells, which were measured throughout the experiment, was assumed to represent the hydrautic head along the circular outer boundary. Within the circular area, it was assumed that (1) the aquifer is confined and homogeneous, and (2) the confining bed is impermeable. Given the above boundaries and boundary conditions, and the parameters in Table 13, the experiment was simulated.

The field experiment suffered from a clogging problem during injection. Backwashing operations were required to restore permeability, but injection pressures still were increased. Eventually, the increased pressure forced 


\section{TABLE 13. Parameters for Heat-Transport Model}

Heat capacity of rock

Medium thermal conductivity

Dispersivity

Overburden thermal conductivity

Aquifer porosity
$1.81 \times 10^{6}$ joules $/ \mathrm{m}^{3}-{ }^{\circ} \mathrm{C}$

$1.98 \times 10^{5}$ joules $/ \mathrm{m}-$ day- ${ }^{\circ} \mathrm{C}$

$1.5 \mathrm{~m}$

$2.21 \times 10^{5}$ joules $/ \mathrm{m}-$ day- ${ }^{\circ} \mathrm{C}$

0.25 
water into overlying formations either through a rupture in the confining bed or through a rupture in the annulus between the well casing and the borehole. Because of these problems, the injection rates had to be idealized.

In spite of these problems, a successful simulation was obtained (see Figures 15 and 16). The energy recovered associated with temperatures above the ambient ground-water temperatures was predicted to be about $3.8 \times 10$ joules, which is about $15 \%$ higher than that measured. This difference, which resulted in a predicted recovery ratio of 0.75 versus a measured value of 0.69 , is primarily due to the problems associated with performing the experiment.

During the second phase of the Mobile experiment, clogging was still a problem, but several improvements in the experimental techniques were made that helped overcome the severe problem of the first phase. These improvements consisted of (1) supply water being pumped from a sandy-gravel aquifer instead of using surface water and (2) using a regular well backwashing program.

The two cycles of the second phase were simulated by Tsang et al. (1980b) using the code CCC. For this simulation, the impermeable boundary determined by Papadopulos and Larson was neglected, and the problem was again simulated with a cylindrical grid. Many of the parameters used were similar to those used by Papadopulos and Larson (1978). Because neither the injection flow rate nor temperature was held constant, it was necessary to treat injection and production as segments having average flow rate and temperature values, but conserving injected mass and energy.

For the first cycle, the simulated recovery factor was 0.68 , which compares well with the observed value of 0.66 . For the second cycle, the simulated value was 0.78 , compared to an observed value of 0.76 . For both cycles, the initial simulated and observed temperature decreases slightly faster than the simulated temperature. During the latter part, the simulated temperature increases faster than the observed temperature so that by the end of the production period the simulated and observed temperatures again agree $\left(33^{\circ} \mathrm{C}\right)$. The discrepancy over the whole range has a maximum value of 1 to 2 degrees. 


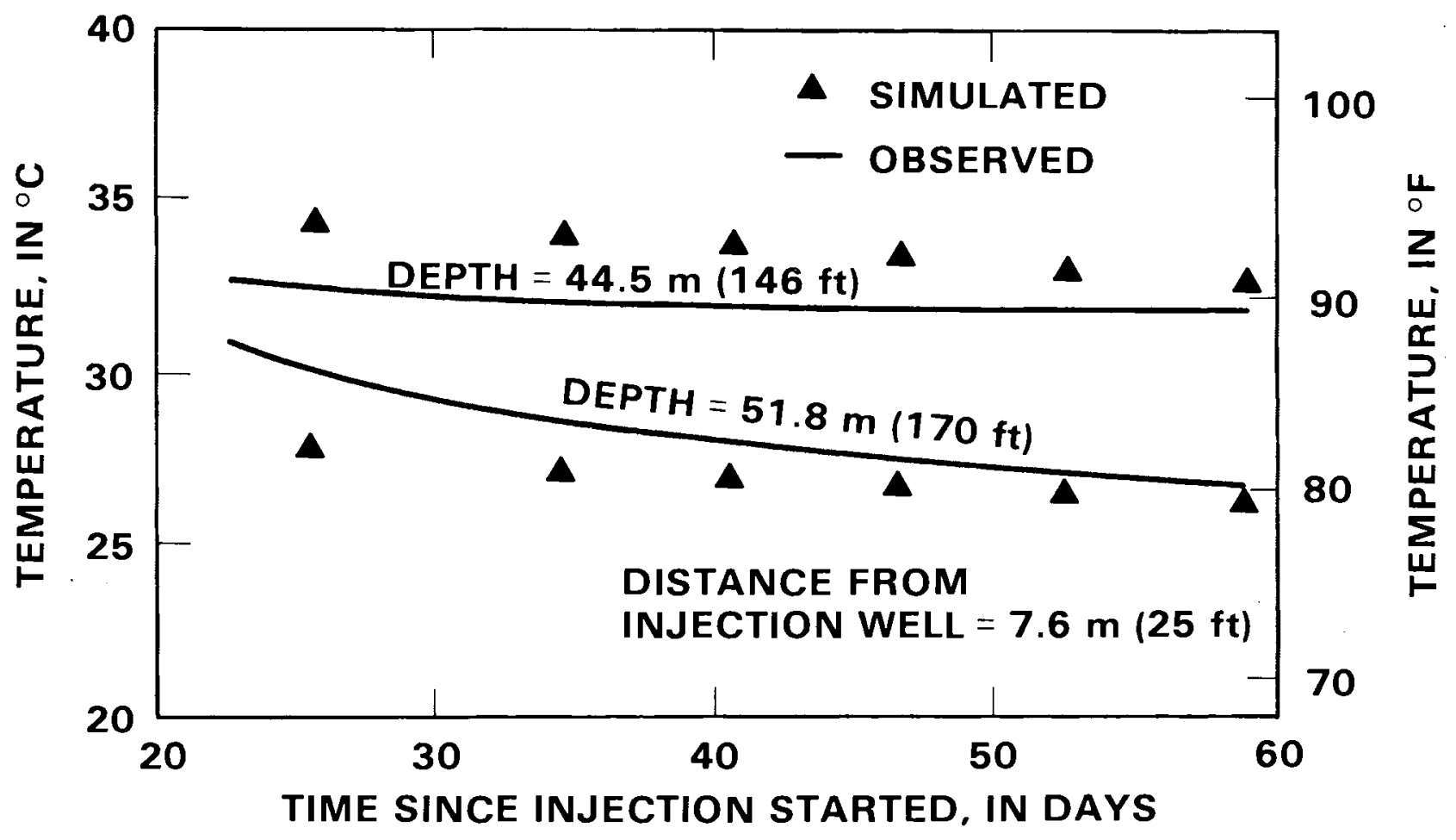

FIGURE 15. Comparison of Simulated and Observed Temperature History at Selected Depths in Well 1

(from Papadopulos and Larson 1978) 


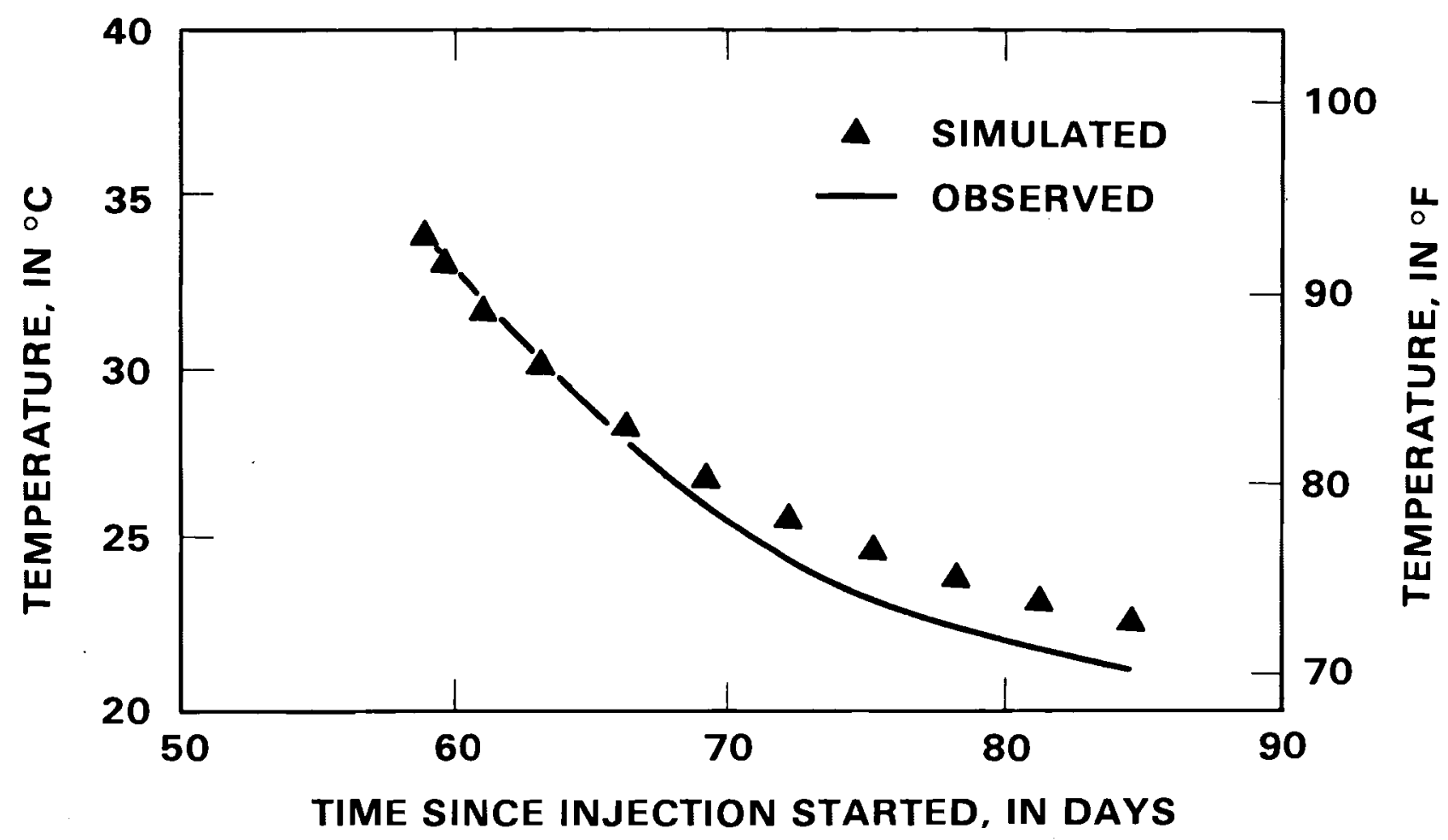

FIGURE 16. Comparison of Simulated and Observed Temperatures

of the Recovered Water

(from Papadopulos and Larson 1978) 


\section{Bonnaud, France}

\section{Location}

This ATES site is located at the Bonnaud Site in Jura, France, which is north of Lyon. The experiments to be discussed were performed by the Bureau de Recherches Géologiques et Minières, Orléans, France together with the Centre d'Études Nucléaires de Grenoble and the École des Mines de Paris (Tsang 1979). The experiments were performed in 1976 and 1977.

\section{Hydrogeology}

The storage aquifer was a shallow, confined sand and gravel $2.5 \mathrm{~m}$ thick. Details concerning aquifer hydraulic properties and water levels were not reported in Tsang (1979), Tsang et al. (1980a), or Sauty et al. (1978). The ambient ground water temperature was $12^{\circ} \mathrm{C}$ (Tsang, 1979).

\section{Description of Field Operations}

The Bonnaud experiments included constant rate injection of hot water in one well and production at the same rate from another well (doublet), and two series of heat storage experiments from a central well (Sauty et al. 1978). The first series of single well heat storage experiments consisted of three successive injection and production cycles during 1976. The second series of single well experiments consisted of four injection/production cycles during 1977 (Tsang et al. 1980a). Information regarding the doublet field experiment was not reported in Sauty et a1. (1978), Tsang (1979), or Tsang et al. (1980a). Only details of the first cycle of the first series of the single well heat storage experiments will be described as in Tsang (1979) and Tsang et al. (1980a).

In the first cycle of the first series, a total of $1,400 \mathrm{~m}^{3}$ of water at $40^{\circ} \mathrm{C}$ was injected over a 20 day period and stored for 122 days. After storage, $3,000 \mathrm{~m}^{3}$ of water was withdrawn for thermal energy recovery. The energy recovery ratio for this cycle was 30\% (Tsang, 1979; Tsang et al. 1980a). 


\section{Discussion}

The data from the 1976 single well experiment were used to calibrate a numerical model in order to predict the results of the 1977 experiments. The model used was four-layered and axisymmetric, and the numerical code, called ESTHER, was verified against an analytical solution derived by Rubinstein (1972). According to Sauty et al. (1978), it accurately predicted the 1977 experimental results except for some minor differences during injection. These differences were attributed to the fact that in the model the average temperature is calculated in a cylindrical node with a $2.7 \mathrm{~m}$ radius while in the field experiment, the temperature was measured inside the well (Sauty et al. 1978). Also, the real system was slightly anisotropic, while the model simulated an average thermal behavior as a function of distance from the central we11. This averaging of heterogeneities caused the apparent thermal conductivities to be much higher than the actual thermal conductivities.

In addition, a three-dimensional model was constructed and calibrated against both the single and doublet well storage experiments. Exact details of this model were not reported in Tsang (1979), Tsang et al. (1980a), or Sauty et al. (1978). However, Sauty et al. (1978) did report that the physical parameters used in the calibration of the three-dimensional mode 1 agreed well with those used in the axisymmetric model. He further indicated that the non-axisymmetric distributions of heat and temperature for the single well experiments were due to transmissivity heterogeneities.

Overall, the results give strong evidence of heat dispersion during injection and recovery with the resulting apparent thermal conductivities in the aquifer being much higher than the actual thermal conductivities (ATES Newsletter, January 1979).

\section{Gard, France}

\section{Location}

This ATES site is located at Gard, France, and is known as the Campuget experiment. The research was sponsored by the Ministère de l'Équipement, Plan Construction, and carried out jointly by Électricité 
de France, Direction des Études et Recherches, Départment Application de l'Électricité, and École des Mines de Paris, Centre d'Informatique Géologique, Fontainebleau (Cormary et a1. 1978). The field test began on July 3, 1977 and was terminated on March 15, 1978 (Cormary et al. 1978).

\section{Hydrogeology}

The storage aquifer was a shallow unconsolidated phreatic aquifer $7 \mathrm{~m}$ thick. Its hydrautic conductivity was given as $1.0 \times 10^{-3} \mathrm{~m} / \mathrm{s}$. An active pumping wel1 $200 \mathrm{~m}$ from the injection wel1 caused a $2 \%$ hydraulic gradient, and the depth to water ranged from 0.3-0.9 m during the course of the study (Cormary et a1. 1978).

\section{Description of Field Operations}

This experiment was a full scale hot-water storage project. It consisted of five stages which were:

(1) The injection of $20,200 \mathrm{~m}^{3}$ of water averaging $33.5^{\circ} \mathrm{C}$ between July 3, 1977 and September 28, 1977. The water was withdrawn from the same aquifer being used for storage through the pumping well previously mentioned in the hydrogeologic description. After withdrawal, the water was heated by solar energy and injected into the aquifer at a depth of 7 to $10 \mathrm{~m}$.

(2) A storage period lasting from September 28, 1977 to November 8, 1977. During this period, the temperature at the center of the heat plume decreased from $33.5^{\circ} \mathrm{C}$ to $30^{\circ} \mathrm{C}$.

(3) The withdrawal of $5,000 \mathrm{~m}^{3}$ of water (apparently from the same well used for injection) at a temperature decreasing from $30^{\circ} \mathrm{C}$ to $21^{\circ} \mathrm{C}$ between November 8, 1977 to December 20, 1977. This withdrawa 7 recovered $14 \%$ of the total heat stored.

(4) A second storage period lasting from December 20, 1977 to January 20, 1978. During this period, the temperature at the center of the plume decreased from $21^{\circ} \mathrm{C}$ to $19^{\circ} \mathrm{C}$.

(5) The withdrawal of $12,000 \mathrm{~m}^{3}$ of water at a temperature decreasing from $19^{\circ} \mathrm{C}$ to $14^{\circ} \mathrm{C}$. This withdrawal recovered $4.5 \%$ of the total heat stored.

Overa11,20,200 $\mathrm{m}^{3}$ of hot water was stored in an aquifer and, in two episodes, $17,000 \mathrm{~m}^{3}$ of the stored water was retrieved. The heat recovered in this experiment represented on $1 y 18.5 \%$ of the total heat stored. 


\section{Discussion}

There were two basic aims of the experiment. The first was a realization of a full-scale interseasonal storage project in a phreatic aquifer including the study of its evolution, the measurement of the efficiency of thermal recovery, and numerical simulations. The second was the utilization of the stored heat in existing greenhouses for space heating applications (Cormary et al. 1978 and ATES Newsletter, October 1978). Both have been accomplished. The experimental procedures were outlined in the previous section and details concerning the space heating applications were not reported in Cormary et al. (1978) or in the ATES Newsletters. A discussion of the efficiency of thermal recovery and of the numerical model follows.

The thermal recovery ratios have been given in the previous section. As reported, a significant decrease in efficiency was observed between the first and second withdrawal periods and the overall heat recovery for the experiment was not very high. This has been attributed to the phreatic nature of the aquifer, the shallow depth to water, precipitation dilution, and to the low air temperatures during the winter (Cormary et al. 1978; Tsang et al. 1980a). The primary heat loss mechanisms determined by computer analysis were (1) thermal dispersion in the aquifer, (2) conduction through the unsaturated zone, and (3) infiltration of rainwater which decreased the thickness of the unsaturated zone. Secondary heat loss mechanisms were regional ground-water flow and conduction to the lower confining layer (Tsang et al. 1980a).

The quantitative interpretation of the experimental results was performed using a finite element model which coupled the ground-water flow equation with the convective-diffusive equation for thermal energy transport. The model is axisymmetric and multi-layered. Good agreement between experimental and numerical results was reported (Cormary et al. 1978 and ATES Newsletter, May 1979).

Overall, it was considered that the thermal recovery efficiency was positive considering the poor experimental setting (i.e., water table too close to land surface) and that the models duplicated reality with good 
precision. It should also be noted that physical problems such as clogging did not pose serious threats. Bacterial growths did develop but were treated successfully with chlorine.

Yamagata Basin, Japan

\section{Location}

This ATES site is located in the Yamagata Basin in Japan. The study to be described was financed by Yonezawa City and the Yamagata prefecture government, and it was performed by the University of Yamagata in conjunction with Nippon Chikusui-Kaihatsu Co., Ltd. and Mayekawa Manufacturing Co., Ltd. The field experiments began on July 16, 1977 (Yokoyama et al. 1978).

\section{Hydrogeology}

The storage aquifer is a leaky confined aquifer composed of alluvial deposits which average about $19 \mathrm{~m}$ thick. The aquifer overlies green tuff rocks and it is interlayered with thin beds of clay and volcanic ash. The hydraulic conductivity was reported as $1.6 \times 10^{-4} \mathrm{~m} / \mathrm{s}$ and the pre-experimental hydraulic gradient was on the order of $10^{-3}$ to the northwest. In the experimental analysis, it was assumed that leakage did not occur (Yokoyama et a1. 1978).

\section{Description of Field Operations}

The test site consisted of two dual-purpose (i.e., injection/production) wells and one observation well. The three wells are positioned equilaterally with each well being $22.4 \mathrm{~m}$ apart from the other two. Basically, the experimental procedure entailed:

(1) production of cold water from the "North" well during the summer,

(2) using the cool water to air condition a commercial building,

(3) sprinkling the spent air conditioner water on the building's $300 \mathrm{~m}^{2}$ roof for heat collection,

(4) filtering the now warm water in a dual media (activated carbon and sand) filtration tower,

(5) further heating the water by a heat exchanger, and

(6) recharging the warm water back to the same aquifer through the "South" well. 
This process was reversed during the winter when warm water produced from the "South" well was used to melt roof top snow. It was then filtered and recharged as cool water through the "North" well (Yokoyama 1978; Tsang 1979; Tsang et al. 1980a).

At least four injection/production periods, interspersed with dormant storage periods, have been reported for this site. Details of only the first two periods were discussed in Tsang et al. (1980a), Tsang (1979), Yokoyama et al. (1978), and in the ATES Newsletter summaries. A summary of the reported information for the injection/production periods is given in Table 14.

In addition to the field experiments, numerical analyses were conducted to evaluate aquifer thermal behavior and doublet well spacing effects. Particular attention was focused on the phenomena of thermal breakthrough from the injection well to the production well. The analysis was accomplished by using an upstream weighted finite-difference model based on

(1) a complex variable potential function describing the water level distribution,

(2) a transient convective-diffusive equation describing the horizontal convection of thermal energy within the aquifer plus the vertical conduction of thermal energy across the aquifer/confining layer interface, and

(3) a transient 3-D diffusive equation describing the conduction of heat within the confining units (Yokoyama et al. 1978).

\section{Discussion}

According to Yokoyama et al. (1978), there was good agreement between experimental and numerical results. The experimental recovery ccefficient (a little less than $40 \%$ ) satisfactorily coincided with the numerical determination supporting the feasibility of seasonal regeneration. It was also noted that in ground-water systems with slow natural flow, as compared to the well flow (forced convection), horizontal and vertical diffusion are insignificant - further supporting the feasibility of seasonal regeneration. Some additional points were that the experiment was a convection dominated problem, and that the concept of buoyancy flow was neglected in the numerical analyses (Yokoyama et al. 1978 and ATES Newsletter, October 1978). 
TABLE 14. Summary of Injection/Production Information

\begin{tabular}{|c|c|c|c|c|c|}
\hline \multirow[b]{2}{*}{ Time Period } & \multicolumn{2}{|c|}{ Pumpage } & \multicolumn{2}{|c|}{$\begin{array}{l}\text { Mean } \\
\text { Temperature }\end{array}$} & \multirow{2}{*}{$\begin{array}{r}\text { Energ } \\
\text { Recove } \\
\text { Ratio } \\
\end{array}$} \\
\hline & $\begin{array}{l}\text { Prod. } \\
\left(\mathrm{m}^{3}\right)\end{array}$ & $\begin{array}{l}\operatorname{Inj} \\
\left(m^{3}\right) \\
\end{array}$ & $\begin{array}{l}\text { Prod. } \\
\left({ }^{\circ} \mathrm{C}\right)\end{array}$ & ${ }^{\mathrm{Inj}} \mathrm{C}$ & \\
\hline $\begin{array}{l}\text { JuTy } 16,1977 \text { to } \\
\text { September } 18,1977\end{array}$ & 9930 & 8843 & 17.1 & 23.7 & $40 \%$ \\
\hline $\begin{array}{l}\text { December } 26,1977 \text { to } \\
\text { March, } 1978\end{array}$ & 11200 & 9430 & 18.3 & 5.3 & $40 \%$ \\
\hline Summer 1978 & & & 14.0 & & \\
\hline
\end{tabular}


College Station, Texas

\section{Location}

This ATES site is located in the alluvial floodplain of the Brazos River in Burleson County, Texas. The experiment began in November 1978 and was completed in the fall of 1980 . The research was sponscred by the U.S. Department of Energy and was performed by the Agricultural and Chemical Engineering Departments of Texas A\&M University (Reddell et al., 1978).

\section{Hydrogeology}

The storage aquifer is an unconfined aquifer in the alluvial floodplain of the Brazos River. The floodplain alluvium reaches a maximum depth of about $85 \mathrm{ft}$, averaging about 50 to $60 \mathrm{ft}$ below land surface. The depth to water varies from less than 10 to more than $30 \mathrm{ft}$. Most of the water levels are about 15 to $25 \mathrm{ft}$ below land surface datum. The transmissivity is reported to be about 650 to $930 \mathrm{~m}^{2} / \mathrm{day}$ and the storage coefficient is about 0.15. The natural water temperature in the aquifer is about 18 to $21^{\circ} \mathrm{C}$. Also, the water quality is not good. It is high in sodium, calcium, chloride, and iron (Reddell et al. 1978).

\section{Description of Field Operations}

This experiment was designed to be a prototype for the injection and storage of chilled water in a ground-water aquifer. The field site consists of two injection/production wells, 12 observation wells, and a $404 \mathrm{~m}^{2}$ cooling pond (Reddell et al. 1978). One of the two injection/production wells is used as a production well to feed the cooling pond while the other injection/ production well is used to inject the cooled water back into the aquifer. Both can be used for either injection or production.

The experiment consisted of two cycles. The first cycle began in November 1978 and ended in the fall of 1979. There were three phases. The first phase began November 1978 and was terminated April 1979. This phase entailed pumping $31,800 \mathrm{~m}^{3}$ of $21^{\circ} \mathrm{C}$ water from the aquifer into the cooling pond, chilling the water to about $10^{\circ} \mathrm{C}$, chlorinating it, filtering it, and then injecting the chilled water back into the aquifer. The second phase 
was a storage period which lasted until June 1979. The third phase followed and lasted into the fall of 1979. It entailed recovering the chilled water from aquifer storage, passing it through a heat exchange process for air conditioning, and then reinjecting the water into the aquifer (Tsang, et a1., 1980a). 23\% of the input energy was recovered.

The second and final cycle duplicated the procedures of the first cycle but with less water and at lower injection temperatures. In the first phase, about $8,100 \mathrm{~m}^{3}$ of water was produced, chilled to about $5^{\circ} \mathrm{C}$, treated, and injected. It was stored until the summer at which time the cool water was produced recovering $31 \%$ of the input energy.

This was the final experiment of this series. However, further chilled water experiments are being planned. The proposed ATES experiments will be located at another site utilizing a confined aquifer. Also, the development of a mathematical model has recently been completed. It is a three-dimensional finite-difference model in radial coordinates that uses an alternating direction implicit matrix solution procedure. It will be used to further evaluate the results of the experiments described.

\section{Discussion}

The aim of this study was to design, develop, and demonstrate a prototype chilled water ATES system (ATES Newsletter, October 1978). This was accomplished in a two cycle experiment. Some initial concerns about the experiment involved the possibility of formation plugging and the effectiveness of the cooling procedure. No evidence of plugging was observed during either cycle and the chilling procedure proved effective especially after modifications permitted chilling the water about $5^{\circ} \mathrm{C}$ for the second cycle. The overall conclusions were that the chilling procedure worked well and that chilled:water ATES.can be feasible for air conditioning purposes. 


\section{Minneapolis-St. Paul, Minnesota}

\section{Location}

This ATES site is located on the St. Paul campus of the University of M nnesota at Minneapolis-St. Paul, Minnesota. The project is being funded by Pacific Northwest Laboratory for the U.S. Department of Energy and is being performed by the University of Minnesota with other major participants being Honeywe1l Technology Center, G.E. Tempo, the U.S. Geological Survey, the Minnesota Geological Survey, and the Minnesota Energy Agency (ATES Newsletter, March 1980). It is still in the initial stages of aquifer characterization via (1) preliminary flow and energy transport modeling, (2) geophysical logging, and (3) hydraulic testing of core holes. Initial results should be available in the summer of 1981 (ATES Newsletter, June 1980).

Hydrogeology

The intended storage medium is the Franconia-Ironton-Galesville sandstone aquifer system. This system ranges in depth from about 610 to a little over $800 \mathrm{ft}$ below land surface. It is a confined system with the Eau Claire and St. Lawrence Formations forming the basal and upper confining units, respectively.

\section{Description of Field Operations}

The plan for this demonstration project is to use co-generated steam from the University heating plant as an energy source. A doublet well system will be used to store high temperature $\left(150^{\circ} \mathrm{C}\right)$ water in the FranconiaIronton-Galesville aquifer system, and to later recover it for district heating. Actual thermal energy storage had not begun as of November 1980. However, test hole drilling and the geophysical logging of the test holes was complete. The data from the drilling and logging have been used in preliminary flow and energy transport models. 
Stony Brook, New York

\section{Location}

This ATES site is located on the campus of the State University of New York (SUNY) at Stony Brook, New York. The prime contractor performing this project is Dames \& Moore of Cranford, New Jersey. Other participants include SUNY at Stony Brook, the engineering firm of Ambrosino and DePinto, and the New York State ERDA. The project is being funded by Pacific Northwest Laboratory for the U.S. Department of Energy and by the New York State ERDA. It is currently in progress. As of September 1980, the well drilling and initial aquifer characterization were being performed (STES Newsletter, September 1980).

\section{Hydrogeology}

The intended storage aquifer is the Magothy Aquifer, which is a part of the Atlantic Coastal Plain. By the end of September 1980, two $122 \mathrm{~m}$ deep monitoring wells were completed and work was proceeding in the drilling of the remaining monitoring wells and the second doublet well. The geohydrologic data obtained from logging the newly completed holes and that from the preexisting well (to be used as one of the doublet we11s) correspond. Also, aquifer tests on the pre-existing well have indicated satisfactory yields. However, information regarding the hydraulic properties of the recently drilled wells has not been published (STES Newsletter, 1980).

\section{Description of Field Operations}

Dames \& Moore, Inc. are investigating the possibility of storing chilled water in the Magothy Aquifer underlying SUNY at Stony Brook, New York. The conceptual design is to use an existing cooling tower to chill aquifer water in the winter, to re-inject the chilled water, and then to produce it in the summer into a pre-existing chilled water distribution system for cooling several campus buildings. As of September 1980, the actual storuge of chilled water had not occurred. However, mathematical model preparation has been performed (STES Newsletter, September 1980). The model, GREASE2, has been adapted and verified against classical field 
problems and analytical solutions, and finite element meshes for the well doublet simulation have been constructed. The analysis and simulation of the aquifer test data was to be performed in the last quarter of 1980 followed by an analysis of alternative well field designs.

Bethel, Alaska

\section{Location}

This ATES site is located on the Kuskokwim River at Bethel, Alaska. The prime contractor performing the study is TRW, Inc. of Redondo Beach, California. Other participants include Harding and Lawson, Inc., WoodwardClyde, R and M Consultants, the Calista Corporation, the Bethel Utility Corporation, and the Bethel Hospital. It is being funded by Pacific Northwest Laboratory for the U.S. Department of Energy. As of September 1980 , it was in the initial stages of well drilling and aquifer hydraulic characterization (STES Newsletter, September 1980).

\section{Hydrogeology}

Not much is known, as yet, about the aquifer in the vicinity of the test site. The site is on the deltaic deposits of the Kuskokwim River which are underlain by about $122 \mathrm{~m}$ of permafrost. The aquifer is thought to be about 30 to $61 \mathrm{~m}$ thick, and to lie somewhere between 122 to $244 \mathrm{~m}$ below land surface (STES Newsletter, September 1980).

\section{Description of Field Operations}

The plan for this demonstration project is to use waste heat from the diesel generator power plant, owned and operated by the Bethel Utility Corporation, for the district heating of the town of Bethel. Any excess heat, in the form of hot water, will be placed in aquifer storage. The storage site will consist of five wells; two will constitute an injection/ production doublet and the other three will be used for observation wells. 


\section{AQUIFER CHARACTERISTICS}

The utilization of models in an ATES project will be application dependent. A major factor affecting the modeling approach will be the aquifer character. An ideal aquifer for ATES is difficult to define due to the many physical factors that must be considered. For example, a thin aquifer is more suitable for minimizing the tilting of a thermal front; however, a thin aquifer wi11 probably have greater heat losses. Table 15 provides a summary of some of the desirable characteristics of an ATES site, and circumstances that are favorable and unfavorable. In all cases, a low natural gradient is desirable.

To overcome deficiencies inherent in most aquifers considered for ATES requires an operating approach that minimizes the negative effects and maximizes thermal recovery. Models can be used for this operation design (see, for example, Sauty et a1. 1978). In the following discussion we discuss the use of models for several different types of aquifers including:

- shallow water-table aquifers

- deep water-table aquifers

- thin confined aquifers

- thick confined aquifers

- heterogeneous aquifers

- fissured or cavernous aquifers

Shälow Water-Table Aquifers

Shallow water-table aquifers are generally unsuitable for ATES projects. Special consideration must be given to heat lost to the surface or unsaturated zone above the aquifer. If the aquifer is highly permeable, tilting of the thermal front can lead to poor recovery. If the permeability is low, sufficient volumes of water cannot be injected without causing unacceptable buildup in the water table. An additional problem is the possibility of excessive recharge that could dilute the heated or cooled water.

To analyze the control of thermal tilting requires the use of crosssectional or three-dimensional models. The analytical solutions summarized in a previous section can be used to provide preliminary estimates of the tilting effect. 
TABLE 15. Desirable Characteristics of an ATES Project and Corresponding Favorable and Unfavorable Aquifer Conditions

Desired

Character

Lack of thermal tilting

Low heat loss to

confining beds

Low pumping and

injection costs

Rapid heat exchange

between fluid and rock

Low heat loss to surface

Minimum therna 1

dispersion

Lack of regional flow
Favorable

Condition

Thin aquifer

Low vertical permeability

Moderate horizontal permeability

Thick aquifer

High horizontal permeability

High porosity

Fine grains

Homogeneous conditions

Deep confined aquifer

Homogeneous aquifer

Low regional hydraulic head gradient (i.e., low pore water velocity)
Unfavorable Condition

Thick aquifer High vertical permeability

Thin aquifer

Low horizontal permeability

Heterogeneous conditions

Shallow watertable aquifer

Highly heterogeneous aquifer

High regional hydraulic head gradient (i.e., high pore water velocity) 
Water-table build-up due to injection or recharge can be evaluated using two-dimensional, areal models. If the aquifer is thin, large variations in the water-table position will be difficult to simulate because the governing equations will be strongly nonlinear. Furthermore, in a thin shallow aquifer the concept of a water table can be a too simplified approach. If this is the case, it may be necessary to use a non-isothermal, saturated-unsaturated flow model (which presently only exist as research tools).

Deep Water-Table Aquifers

Deep water-table aquifers, though far from ideal, are not as limited as shallow water-table aquifers. Difficulties with heat loss to the surface and water-level build-up are generally not as severe. But, because the deeper aquifers are usually thicker, the potential for tilting of the thermal front is greater. If tilting of the front is important, it will necessitate the use of cross-sectional, axisymmetric or three-dimensional models. Unfortunately, symmetry for the cross-sectional or axisymmetric models may not exist. Thin Confined Aquifers

Thin confined aquifers while less prone to tilting of a thermal front are apt to lose significant heat to confining beds. For thin aquifers, it is important that the heat loss and fluid loss (or gain) to confining beds be properly treated. Usually a two-dimensional, areal model will be appropriate if leakage to confining beds is included. Because heat loss will tend to diffuse the front, numerical dispersion will not be a major concern. Thick Confined Aquifers

"Moderately" thick confined aquifers with a "moderately" low permeability are desirable for ATES projects. A thick aquifer having high permeability and a low ratio of horizontal to vertical anisotropy will be susceptible to tilting of the thermal front. For those cases, a cross-sectional, axisymmetric or three-dimensional model should be used. Again, the axisymmetric and cross-sectional models will be useful if the required symmetry is present.

From a thermal recovery perspective, it is desirable to have a homogeneous media. Such media do not produce high thermal dispersion of the front. Without the additional dissipative mechanism of high heat loss to 
confining beds, the thermal front can be confined to a thinner zone. This can lead to important modeling difficulties--numerical dispersion or oscillation.

Heterogeneous Aquifers

Heterogeneous aqlifers are usually less desirable than more uniform ones due to greater thermal dispersion. However, one common type of heterogeneity is beneficial because it reduces the potential for tilting of the thermal front. This type of heterogeneity occurs in stratified material with thin layers of lowly permeable sediments interbedded with more permeable 1ayers. Despois and Nougarede (1979) provide a good discussion of the effects of heterogeneity on ATES.

From a modeling point of view, heterogeneity is both good and bad. It is good in that the larger natural thermal dispersion reduces the errors caused by numerical dispersion or oscillation. It is bad in that field data will generally be more erratic and difficult to compare with model results. For some applications in which different strata are thick and readily identified, it may be necessary to use a cross-sectional, axisymmetric, or threedimensional model. For other cases, a two-dimensional, areal model may be sufficient.

Fissured or Cavernous Aquifers

As noted in an earlier section, fissured or cavernous aquifers are usually unsuitable for ATES projects. The primary exception being pervasively fractured media that in effect behaves as porous media.

To illustrate the limitations of fractured media, consider the case of a single fracture zone in a relatively impermeable rock. This is the case of a thin aquifer in the extreme. As a first approximation, the physical situation can be described by the analytical solution of Lauwerier (1955). Using thermal properties typical of igneous rocks and assuming a fracture zone having a porosity of 0.3 , the results shown in Figure 17 were calculated for an injection period of $10^{7}$ seconds. As can be seen for fracture zones less than a few meters thick, the loss of heat to the surrounding rock is 


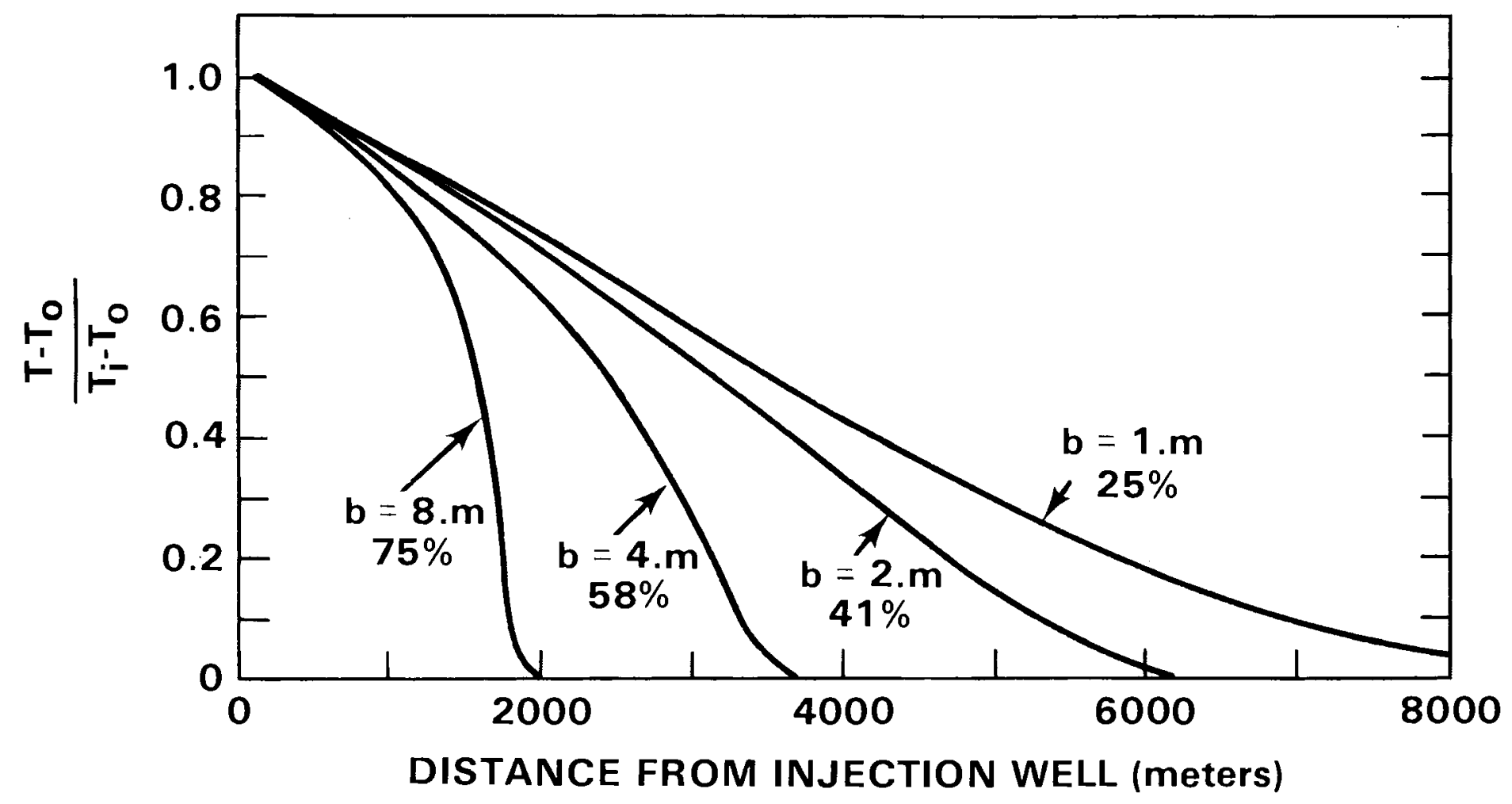

FIGURE 17. Example Results Showing the Effect of Fracture Zone Thickness (b) on Temperatures in Fracture and Percent of Heat Retained in the Fracture Zone 
high. For fracture zones thinner than considered in this analysis, the heat loss would be much greater.

For highly fissured aquifers, a porous media model will be appropriate. The previous discussion of aquifer types and modeling ccnsiderations is applicable to similar aquifer conditions in the pervasively fissured rocks.

\section{FLUID AND ROCK PROPERTIES}

The governing equations for fluid flow, heat transport, and solute transport include many parameters. In general, these parameters represent physical properties of the fluid or aquifer rock. In this section, we consider the parameters that are required for models. The remaining discussion emphasizes sources of data and the uncertainty associated with it.

For ground-water flow important parameters are:

fluid: $-\rho \ldots$ density, $\left(\mathrm{ML}^{-3}\right)$

- $\mu \ldots$ dynamic viscosity, $\left(\mathrm{ML}^{-1} \mathrm{t}^{-1}\right)$

- $\beta_{T} \ldots$ coefficient of thermal expansion, $\left(T^{-1}\right)$

- $\beta_{\mathrm{p}}$.... coefficient of fluid compressibility, $\left(\mathrm{ML}^{-1} \mathrm{t}^{-2}\right)$

rock: $\cdot \overline{\bar{k}}$... intrinsic permeability tensor, $\left(L^{2}\right)$

- $\phi . .$. porosity, (dimensionless)

- $\alpha \ldots$ intergranular vertical compressibility, $\left(\mathrm{M}^{-1} \mathrm{LT}^{2}\right)$

Additional properties required for heat transport include:

fluid: $-c_{p} \ldots$ heat capacity at constant pressure, $\left(\mathrm{L}^{2} \mathrm{t}^{-2} \mathrm{~T}^{-1}\right)$

rock: ${ }_{-}^{c} \mathrm{pr}$.. heat capacity at constant pressure, $\left(\mathrm{L}^{2} \mathrm{t}^{-2} \mathrm{~T}^{-1}\right)$

- $\overline{\bar{K}}_{\mathrm{m}}^{\star} \ldots$.... medium conduction/dispersion tensor, $\left(\mathrm{MLt}^{-3} \mathrm{~T}^{-1}\right)$

And for solute transport:

rock: $\cdot \bar{D} \ldots$ dispersion tensor, $\left(\mathrm{L}^{2} \mathrm{t}^{-1}\right)$

For the most general case, all of the above parameters are considered functions of temperature and pressure. For temperature variations found in ATES, this dependence is small and many of the parameters may be treated as constants. For projects involving temperature variations of $50^{\circ} \mathrm{C}$, the dependence on temperature should be included for at least the fluid properties. 
The fluid properties of pure water are we1l known and documented (see for example, Keenan and Keys 1969; Meyer et a1. 1968). The uncertainty in this data is extremely sma11 in comparison to that associated with rock properties. Even if the water has significant dissolved impurities, deviations from pure-water data are usually sma11.

Three types of rock properties can be determined with reasonable accuracy by laboratory measurements--porosity, heat capacity, and thermal conductivity. Representative data for various rock types can also be found in standard references. Freeze and Cherry (1979), for example, provide data on porosity for typical aquifer materials. The heat capacity and thermal conductivity of numerous samples are summarized by Clark (1966). Values of these parameters used in models associated with field applications previously discussed are summarized in Table 16. While not insignificant, the uncertainty associated with these parameters is of less concern than that associated with the properties yet to be discussed.

The remaining aquifer or rock properties are generally estimated from field data. Although $\alpha, \overline{\hat{k}}$, $\overline{\bar{D}}$, and $\overline{\bar{K}}_{m}^{\star}$ can be measured in the $1 a b$, the results usually do not correlate well with field-determined values. Each of these four parameters will have considerable uncertainty associated with it.

The permeability, $\overline{\bar{k}}$, and aquifer compressibility, a are determined from aquifer tests. Standard tests provide estimates of the composite parameters transmissivity and storage coefficient. Recall that transmissivity is the product of the average horizontal permeability and the aquifer thickness. Vertical permeability can be a very important consideration in areas where tilting of the thermal front is anticipated. The vertical component of the permeability tensor is more difficult to determine and it is subject to greater uncertainty.

Thermal and mechanical dispersion, $K_{m}^{\star}$ and $D$ are determined by thermal injection and tracer tests, respectively. In contrast to aquifer tests that can be conducted in a few days, these tests require much more time (from a few weeks to several months). If the aquifer is relatively 
TABLE 16. Reported Parameters Used for Field Applications

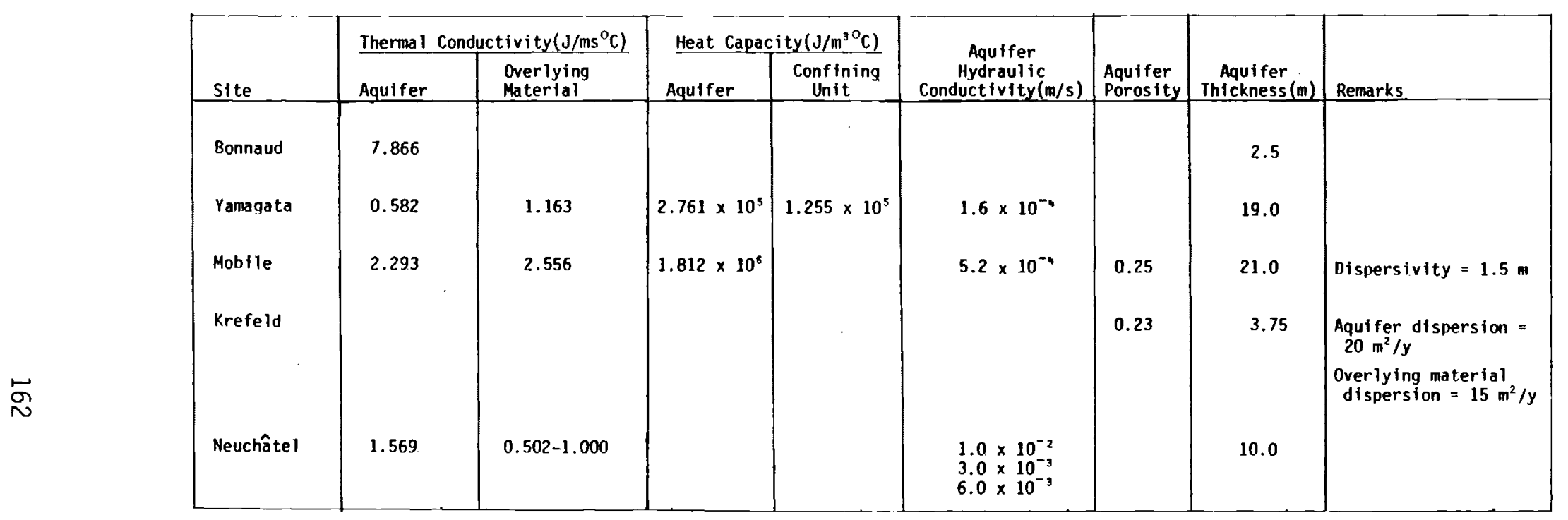


homogeneous and the wells penetrate and are screened over the aquifer's entire thickness, then analytical solutions exist to determine dispersivities (see for example, Sauty 1980 for a discussion of tracer tests and methods of analysis). Interpretation of the field dispersion terms must be done with caution as it is often observed that the field value of dispersivity is dependent on the scale of the field test. Sauty et al. (1978) show results for hot-water storage, which indicate that thermal dispersion approaches a uniform value at larger distances where local heterogeneities have less influence.

In the most general case, the field parameters $\alpha, \overline{\bar{k}}, \bar{K}_{m}^{\star}$, and $\overline{\bar{D}}$ can vary from point to point in the aquifer. Although the analytical solutions do not strictly apply to heterogeneous systems, they can be used as a first approximation. For permeability and aquifer compressibility, aquifer tests at multiple wells can provide a measure of spatial variability. For thermal and mechanical dispersion, multiple tests would be extremely expensive and time consuming. The uncertainty associated with the spatial variability of important parameters is very high in heterogeneous systems.

Because of the limiting assumption of many of the analytical solutions, numerical models are being used more frequently to analyze field tests. Although the numerical model enables a more complex analysis, the possibility of generating meaningless parameter values is also greater. The parameter estimates for complex models will be non-unique. Consequently, parameters that have little influence on the solution can be assigned unrealistic values.

BOUNDARY AND INITIAL CONDITIONS

In order to complete the description of a particular site, it is necessary to specify the initial and boundary conditions. Data for boundary and initial conditions is obtained in the field. Often relevant data exist in published form as hydrologic reports, geologic reports, geologic maps, and topographic maps. This data should be supplemented with studies at the site. Data from wells (cores, geologic logs, geophysical logs, water-level measurements, temperature measurements and concentration measurements) are the most direct. Indirect methods such as surface resistivity, seismic surveys, and remote sensing can extend the data base in a qualitative manner. 


\section{USES AND LIMITATIONS OF TRANSPORT MODELS}

To properly utilize aquifers as a storage medium for heat requires an approach for understanding and predicting the behavior of the relevant physical and chemical processes. Mathematical models are the most general tool for achieving these dual purposes. Because the relevant physical and chemical processes are complex, the models will often require the use of numerical methods. For certain applications and theoretical analysis, simple analytical models may be sufficient.

Models have been used in several ways for ATES. These include generic sensitivity analysis, site-specific aquifer characterization, and sitespecific predictive analysis. Most of the studies have focused on the first two as a means to gain a better understanding of the important processes in general or on a site-specific basis. Whereas generic studies have been useful in evaluating the general potential for ATES, progress in the utilization of ATES will require greater emphasis on site-specific applications.

\section{SITE-SPECIFIC MODEL USE}

Before applying models to an ATES site, it is first necessary to select the models to be used. This decision will be based on important processes, on data availability, and on management objectives. For example, in a particular aquifer, solute transport might be only a minor concern and, consequently, not modeled. The assessment of an aquifer for ATES will generally require a computer model; however, study objectives may be oriented such that a numerical model is unnecessary. Or, if necessary, objectives may require only a very simple model. Additionally, lack of data may not justify a sophisticated model.

The application of models to the assessment of a particular aquifer for ATES will normally have three main phases. These are: system conceptualization, history matching or model calibration, and prediction. A schematic of the interaction and components of the three phases is shown 
in Figure 18. Most applications involve each of the three phases; however, the relative effort devoted to each phase is application dependent.

System conceptualization involves organizing available information on the ground-water system in an internally consistent framework. Sources of information include: (1) published reports on the geology and hydrology of the system; (2) geologic and topographic maps and areal photographs; and (3) unpublished data such as field data, drillers' logs, and geophysical survey data. To complete the conceptualization task, the information is posed in terms of a qualitative model. The qualitative model is translated into mathematical terms such as boundary conditions, initial conditions, and hydrologic parameters. An appropriate ground-water model, which is quantitative, can then be applied to improve the qualitative model. The use of the model involves checking various observations and determining the sensitivity of system responses to hydrologic parameters and assumptions. The results of the system conceptualization should provide a basis for designing data collection efforts. For example, from a thermal injection simulation, the spacing of monitoring wells to observe movement of the thermal front may be determined. Use of a model at this stage not only forces the hydrologist to conceptualize within a tested framework, but also gives guidance in terms of data requirements.

History matching or model calibration is used to refine estimates of hydrologic and thermal parameters and boundary conditions. This is accomplished by comparing model results with observed data. In the early part of the study, observed data will consist of aquifer tests and thermal injection-extraction tests. Estimates of parameters are changed to improve the comparison. It is important to constrain the changes so that physically realistic parameters are specified. This generally requires an experienced ground-water hydrologist. The history-matching procedure can be done by either a trial and error approach or by an automatic regression approach. No matter which approach is selected, sensitivity analysis should be part of the history-matching phase. This sensitivity analysis will be more meaningful than that performed during the conceptualization phase because 


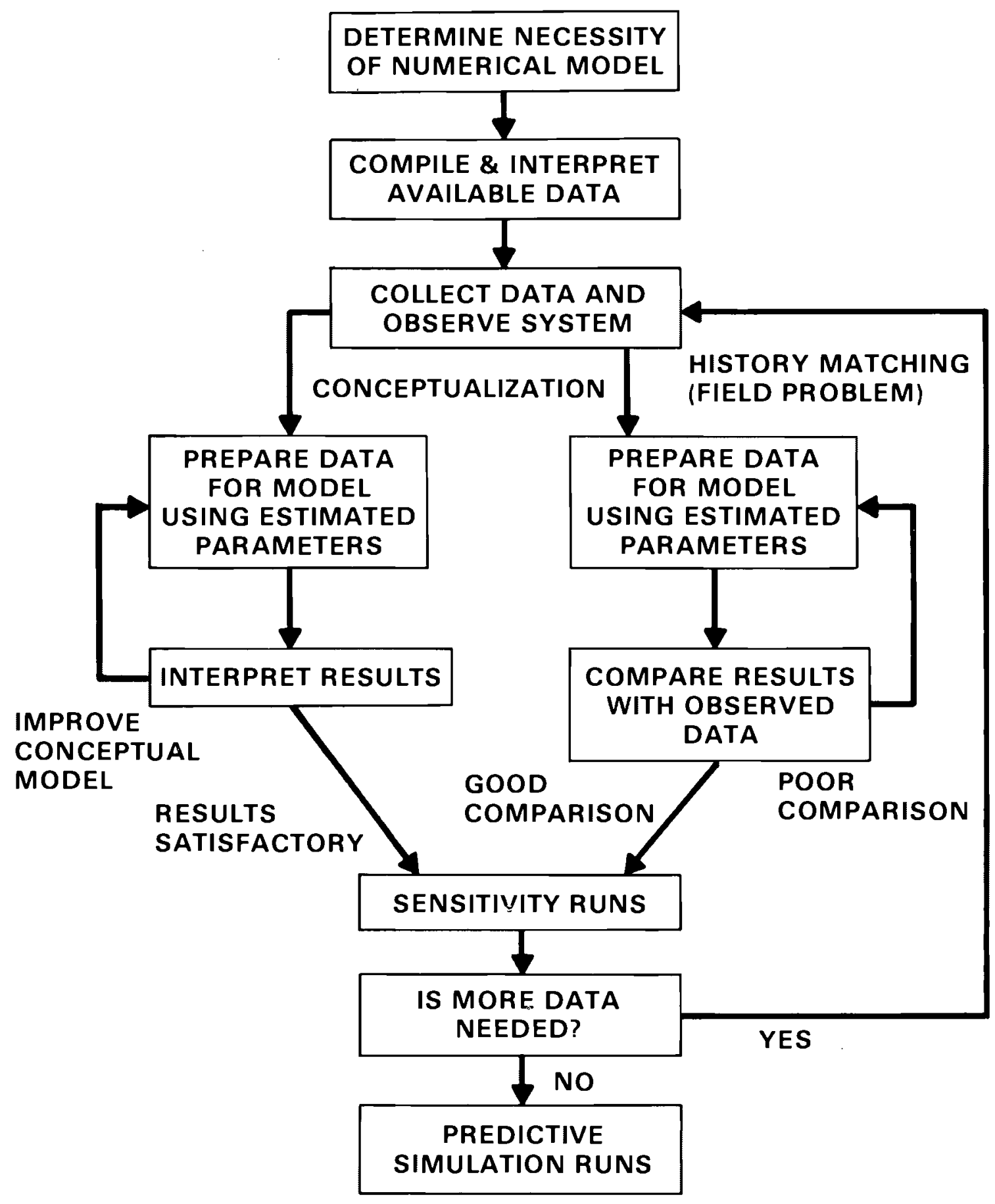

FIGURE 18. Flow Chart Showing Model Use (from Mercer and Faust 1980) 
it is based on more data. Again, the sensitivity analysis should serve as a guide in continuing data collection activities.

The final phase of modeling provides predictions on future system behavior. This is normally the shortest part of a study. Predictions are based on model results using the best estimates of system parameters obtained by history matching. Because the set of parameters is not unique, it is important to assess the uncertainty in the predicted results. This is usually accomplished by again using a sensitivity analysis.

The models discussed in the earlier sections are generally adequate to perform the analyses discussed in this section. Depending on how one designs the problem, these same codes may be used to examine near-well performance as well as environmental effects away from the well. Because of ATES design, both of these problems are considered local, as opposed to a regional study that encompasses several well fields over an area of tens to hundreds of square kilometers.

Although the literature review revealed nothing about the cost of using numerical models for ATES, it has been our experience that computer costs are a very small portion of the total cost of a project. Mantime costs are generally much more expensive than the computer-related costs. This is especially true in analyzing complicated field problems, where data reduction and interpretation are very time consuming.

\section{MODEL LIMITATIONS}

The validity and utility of model results are limited by several factors. In order of importance, these are:

- model misuse

- data uncertainty

- inadequate representation of physical or chemical processes

- numerical approximation and round-off error

Inappropriate model use is the major factor that contributes to a lack of credibility in model results. This misuse is usually related to the experience of the user. Transport models will generally be applied to ATES 
problems. These codes are often complicated and difficult to use, requiring the user to be highly trained. To avoid misuse of these models, one should have a thorough understanding of the physics and chemistry of ATES, as well as numerical analysis and computer techniques. Three common and related ways in which models are misused include: overki11, inappropriate prediction, and misinterpretation.

The temptation to apply the most sophisticated tool is difficult to resist. For ATES application, the use of a fully three-dimensional flow and heat transport model may be possible, but unwarranted. Data requirements for such a model may be excessive and unavailable. For other applications a three-dimensional model may be necessary, but only in the later stages of a study. Even in this situation, if the complex model is applied too early in the study, it may lead to results that are difficult to interpret and are misleading. One must assess the complexities of the problem, the amount of data available, and the objectives of the analysis, and then determine the best approach for the particular situation. In general, one should start with a simple model and a coarse aquifer description. As data and the understanding of the site improve, more complex and detailed models can then be used.

Inappropriate prediction is usually the result of a limited history matching period. One must always be aware that the history-matching portion of the simulation occurred under a given set of field conditions, and that these conditions are subject to change during the predictive portion. Because the models deal with the subsurface, there are always unknown factors that could affect results. In general, one should not predict more than about twice the period used for matching, and only then under similar conditions of system stresses.

Perhaps the worst possible misuse of a model is blind faith in model results. Calculations that contradict normal hydrologic intuition almost always are the result of some data entry mistake, a "bug" in the computer program, or misapplication of the model to a problem for which it was not designed. Proper interpretation of model results requires an understanding of the processes active in and the characteristics of the particular site. 
In addition to model misuse, data limitation can also lead to an improper aquifer characterization. Error caused by erroneous aquifer characterization data is difficult to assess since the true aquifer description is never known. A combination of core analysis, aquifer tests, and geological studies often give valuable insight into the nature of aquifer characteristics. As indicated, the final parameters that characterize the aquifer are usually determined by obtaining the best agreement between calculated and observed aquifer behavior during some historical period. For ATES, this historical period will usually be of short duration, therefore reducing credibility in long-range predictions.

Other limitations to model use are the assumptions on which the model is based. No matter how complex, a model is always less detailed than the system it attempts to represent. Sometimes the simplifications are invoked for convenience; other times they are made because the processes are poorly understood. Even with these approximations, it seems that mathematical models, both analytical and numerical, are sufficiently powerful to solve many ATES applications. Unfortunately, the conceptual basis of some of these mathematical models appears to need reinforcement. This is especially true for incorporating chemical and biological reactions into models.

The most easily recognized limitations on models are those due to the numerical approximations and round-off error. These can be analyzed in a straightforward manner. First, replacement of the model differential equations by a set of algebraic equations introduces truncation error; that is, the exact solution of the algebraic equations differs somewhat from the solution of the original differential equations. Second, the exact solution of the algebraic equations is not obtained due to round-off error, as a result of the finite accuracy of computer calculations. The level of truncation error in computed results may be estimated by repeating runs or portions of runs with smaller space and/or time increments. Significant sensitivity of computed results to changes in these increment sizes indicates a significant level of truncation error and the corresponding need for smaller spatial and/or time increments. Compared to the other error sources, round-off error is generally negligible. 


\section{REFERENCES}

Aagarrd, Per. 1979. Thermodynamic and Kinetic Analys is of Silicate Hydrolys is at Low Temperatures and Pressures. Unpublished Ph.D. dissertation, Univ. of Calif., Berkeley, $127 \mathrm{pp}$.

Allendoerfer, C.B., and C.0. Oakley. 1959. Fundamentals of Freshman Mathematics. McGraw-Hill, New York, New York, 475 pp.

Anderson, M.P. 1979. "Using Models to Simulate the Movement of Contaminants Through Ground-Water Flow Systems." Critical Reviews in Environmental Control. $9(2): 97-156$.

Arnøsson, Stefan. 1981. "Mineral Deposition from Icelandic Geothermal Waters: Environmental and Utilization Problems." Journal Petroleum Technology Jan. 1981:181-187.

Avdonin, N.A. 1964. "Some Formulas for Calculating the Temperature Field of a Stratum Subject to Thermal Injection." Nefti'i Gaz. 3:37-41.

Back, W., and J.A. Cherry. 1976. "Chemical Aspects of Present and Future Hydrogeologic Problems." In Advances in Groundwater Hydrology, 153 pp. A.Z. Saleem, ed. American Water Research Association, Minneapol is, Minn.

Back W., B.B. Hanshaw, T.B. Pyle, L.N. Plummer, and E.D. Weidie. 1979. "Geochemical Significance of Groundwater Discharge and Carbonate Solution to the Formation of Caleta Xel Ha, Quintana Roo, Mexico." Water Resources Research. 15:1521-1535.

Ba11, J.W., E.A. Jenne, and D.K. Nordstrom. 1979. "WATEQ2-A Computerized Chemical Model for Trace and Major Element Speciation and Mineral Equilibria of Natural Waters." In Chemical Modeling in Aqueous Systems, pp. 815-835. E.A. Jenne, ed. American Chemical Society Symposium Series 83, Washington, D.C.

Bear, J. 1972. Dynamics of Fluids in Porous Media. American Elsevier, New York, New York, 764 pp.

Bear, J., D. Zaslavsky, and S. Irmay. 1968. Physical Principles of Water Percolation and Seepage. UNESCO, Paris, France, p. 465.

Bedinger, M.S., et al. 1979. The Waters of Hot Springs National Park, Arkansas - Their Nature and Origin. U.S. Geol. Survey Professional Paper 1044-C, p. C1-C33. 
Brown, D.L., and W.D. Silvey. 1977. Artificial Recharge to a FreshwaterSensitive Brackish-Water Sand Aquifer, Norfolk, VA. U.S. Geol. Survey Professional Paper No. 939.

Campbe11, M.D., and J.H. Lehr. 1973. Water We11 Technology. McGraw-Hi11, New York, N.Y., p. 681.

Carslaw, H.S., and J.C. Jaeger. 1959. Conduction of Heat in Solids. Oxford Press. Clarendon, Great Britain, p. 510.

Cheng, P. 1978. "Heat Transfer in Geothermal Systems." Advances in Heat Transfer, 14:1-105.

Claesson, J. 1980. Tilting Rate of a Wide Thermal Front. Notice 1980/4, Department of Mathematical Physics, Lund Institute of Technology, Lund, Sweden.

Claesson, J., et al. 1978. Theoretical Analysis and Computer Simulation of Solid-Fluid Heat Storage Systems in the Ground; Extractions of Earth Heat. Interim report, part I and II, Department of Mathematical Physics and Building Science, Lund Institute of Technology, Lund, Sweden.

Clark, S.P. 1966. Handbook of Physical Constants. Geological Society of America, New York, New York, p. 587.

Coats, K.H. 1977. Geothermal Reservoir Modeling. Päper SPE6892 presented at the 52nd Annual Fall Meeting of the Society of Petroleum Engineers, AIME, Denver, Colorado.

Coats, K.H., et al. 1974. "Three-Dimensional Simulation of Steamflooding, SPE Journal, December.

Code11, R.B., and D.L. Schreiber. 1979. NRC Models for Evaluating the Transport of Radionuclides in Groundwater. In Management of Low-Level Radioactive Waste. Vol. 2, pp. 1193-1212, M.W. Carter et a1. ed.

Collins, R.E. 1961. Flow of Fluids Through Porous Materials. Reinhold Publishing Corporation, New York, New Yor, p. 270.

Collins, R.E., et a1. 1978. High Temperature Underground Thermal Energy Storage. Presented at Thermal Energy Storage in Aquifers Workshop, May 10-12, Berkeley, California.

Cooley, R.L. 1971. "A Finite Difference Method for Variably Saturated Porous Media: Application to a Single Pumping Wel1." Water Resources Research. 7(6):1607-1625.

Cormary, Y., et al. 1978. Heat Storage in a Phreatic Aquifer: Campuget Experiment (Gard, France). LBL-8431, Thermal Energy Storage in Aquifers Workshop Proceedings, Lawrence Berkeley Laboratory, University of Calif. 
Craft, B.C., et al. 1962. Well Design Drilling and Production. PrenticeHall, Inc., Englewood Cliffs, New Jersey, p. 571.

Crichlow, H.B. 1977. Modern Reservoir Engineering - a Simulation Approach. Prentice-Hal1, Englewood Cliffs, New Jersey, p. 354.

Davis, S.N. and R.J.M. DeWiest. 1966. Hydrogeology. John Wiley and Sons, Inc., New York, New York, p. 463.

de Marsily, G. 1978. "Peut-ou Stocker de l'Energie dans le Sol?" Annales des Mines. 1978(4):11-24.

Desai, C.S., and J.F. Abel. 1972. Introduction to the Fin ite Element Method. Van Nostrand Reinhold, New York, New York, p. 4T7.

Despois, J., and F. Nougarede. 1979. Faisabilite du Stockage Souterrain de Chaleur - Tome 1 et 2. Commissariat a l'Energie Atomique, Societe Nationale Elf-Aquitaine, Paris, France.

Donaldson, E.C., and B.A. Baker. 1977. Particle Transport in sandstones. SPE paper 6905 presented at the 52nd Annual Meeting of SPE, Denver, Colorado.

Doughty, C., et al. 1979. Multiple Well Variable Rate Well Test Analysis of Data from the Auburn University Thermal Energy Storage Program. LBL-10194-UC-66a, Lawrence Berkeley Laboratory, University of California.

Ellis, A.J. and W.A.J. Mahon. 1977. Chemistry and Geothermal Systems. Academic Press, London, p. 392.

Eugster, H.P., et a1. 1980. "Mineral Equilibria in the Six Component Sea Water System $\mathrm{Na}-\mathrm{K}-\mathrm{Mg}-\mathrm{Ca}-\mathrm{SO}_{4}-\mathrm{Cl}-\mathrm{H}_{2} \mathrm{O}$, at $25^{\circ} \mathrm{C}$." Geochim Cosmochim Acta. $44: 1335-1347$.

Faroug Ali, S.M. 1970. 0il Recovery By Steam Injection. Producers Publishing Company, Inc., Bradford, Pennsylvania, p. 122.

Faust, C.R., et al. 1980. The DOE Code Comparison Study: Summary of Results for Problem 1. Presented at the Sixth Workshop on Geothermal Reservoir Engineering, Dec. 16-18, Stanford University, California.

Faust, C.R. and J.W. Mercer. 1977. Theoretical Analys is of Fluid Flow and Energy Transport in Hydrothermal systems. U.S. Geological Survey Open File Report 77-60.

Faust, C.R., and J.W. Mercer. 1979a. "Geothermal Reservoir Simulation 1. Mathematical Models for Liquid- and Vapor-Dominated Hydrothermal Systems." Water Resources Research. $15(1): 23-30$. 
Faust, C.R., and J.W. Mercer. 1979b. "Geothermal Reservoir Simulation 2. Numerical Solution Techniques for Liquid- and Vapor-Dominated Hydrothermal Systems." Water Resources Research. 15(1):31-46.

Faust, C.R., and J.W. Mercer. 1980. "Ground-Water Modeling: Recent Developments." Ground Water. 18(6):569-577.

Finalyson, B.A. 1972. The Method of Weighted Residuals and Variational Principles. Academic Press, New York, New Yor, p. 412.

Freeze, R.A., and J.A. Cherry. 1979. Ground Water. Prentice-Ha11, Inc., Engelwood Cliffs, New Jersey, p. 604.

Fried, J.J. 1975. Groundwater Pollution. Elsevier Scientific, Amsterdam, p. 330 .

Gale, J.E. 1977. A Numerical Field and Laboratory Study of Flow in Rocks with Deformable Fractures. Scientific Series No. 72, Inland Waters Directorate, Ottawa, Canada, p. 145.

Garrels, R.M., and C.L. Christ. 1965. Solutions, Minerals, and Equilibria. Harper and Row, New York, New York. p. 450.

Garrels, R.M., and M.E. Thompson. 1962. "A Chemical Model for Sea Water at $25^{\circ} \mathrm{C}$ and One Atmosphere Total Pressure." American Journal Science. 260: 57-66.

Gibson, V.P., and R.D. Singer. 1971. Water Well Manual. Premier Press, Berkeley, Calif., p. 156.

Gringarten, A.C., and J.P. Sauty. 1975. "A Theoretical Study of Heat Extraction for Aquifers with Uniform Regional Flow." Journal of Geophysical Research. 80(35):4956-4962.

Gringarten, A.C., et al. 1975. "Theory of Heat Extraction from Fractured Hot Dry Rock." Journal of Geophysical Research. 80(8):1120-1124.

Gringarten, A.C., et al. 1977. Etude Theorique du Stockage d'Eau Chaude par Puits Unique Aquifere en 1'Absence d'Ecoulement Regional (Programme Esther). Paper RS-576, in Resume des Principaux Resultats Scientifiques et Techniques du Service Geologique National Pour 1977: Orleans, France, Service Bulletin of the Bureau de Recherches Geologiques et Minieres.

Grisak, G.E., and J.F. Pickens. 1981. "An Analytical Solution for Solute Transport Through Fractured Media with Matrix Diffusion." Journal of Hydrology. Vol. 50 .

Grubaugh, E.K. and D.L. Redde 11. 1980. Heat Transport in Ground-Water Systems: Volume 1-Finite Element Mode 1. TR-104, Volume 1. Texas Water Resources Institute, Texas A\&M University. 
Gupta, S.K., et al. 1980. Description and Applications of the FE3DGW and CFEST Three-Dimensional Finite-Element Models. Presented at the Lawrence Berkeley Laboratory Workshop on "Modeling Thermal-Hydrological Flow in Fractured Rock Mass" Feb. 19-20, Berkeley, Calif.

Guyton, W.F. 1946. "Artificial Recharge of Glacial Sand and Gravel with Filtered River Water at Louisville, Kentucky." Economic Geology. XLI (6):644658.

Hanck, J.A. and G. Nekoksa. 1975. Corrosion Rate Monitoring at The Geysers Geothermal Power Plant. Proceedings U.N. Symposium Development Use Geothermal Resources, 2nd. May 3, 1979-1984, San Francisco, Calif.

Hantush, M.S. 1964. "Hydraulics of Wells." In Advances in Hydroscience. pp. 281-432. V.T. Chow, ed., Academic Press, New York, New York.

Hausz, W. 1974. Heat Storage Wells. Presented at Institute Gas Technology Symposium, Efficient Use of Fuels in Process and Manufacturing Industries, April 17, 1974, Chicago, Illinois.

Helgeson, H.C. 1968. "Evaluation of Irreversible Reactions in Geochemical Processes Involving Minerals and Aqueous Solutions - I. Thermodynamic Relations." Geochim. Cosmochim. Acta. 32:853-877.

Helgeson, H.C. 1969. "Thermodynamics of Hydrothermal Systems at Elevated Temperatures and Pressures." American Journal Science. 267:729-804.

Helgeson, H.C. 1979. "Mass Transfer Among Minerals and Hydrothermal Solutions." In Geochemistry of Hydrothermal Ore Deposits, 2nd. edition. H.L. Barnes, ed., John Wiley, New York, New York.

Helgeson, H.C., et al. 1970. "Calculation of Mass Transfer in Geochemical Processes Involving Aqueous Solutions." Geochim. Cosmochim. Acta. 34:569592 .

Helgeson, H.C., et al. 1969. "Evaluation of Irreversible Reactions in Geochemical Processes Involving Minerals and Aqueous Solutions - II. Applications." Geochim. Cosmochim. Acta. 33:455-481.

Hellström, G., 1978. Aquifer Storage Projects in Sweden. LBL-8431, Proceedings, Thermal Energy Storage in Aquifers Workshop, Lawrence Berkeley Laboratory, California.

Hellström, G. 1979. Heat Storage in Eskers: A Numerical Study. Department of Matehmatical Physics, Lund Institute of Technology, Lund, Sweden.

Hellström, G., et al. 1979. Heat Storage in Aguifers. Buoyancy Flow and Thermal Stratification Problems. Lund Institute of Technology, Lund, Sweden. 
Hendrickson, P.L. 1980. Legal and Regulatory Issues Affecting the Aquifer Thermal Energy Storage Concept. PNL-3437. Pacific Northwest Laboratory, Richland, Washington, p.53.

Hewitt, C.H. 1963. "Analytical Techniques for Recognizing Water Sensitive Reservoir Rocks." Journal Petroleum Technology, August:813-818.

Hobba, W.A., Jr., et al. 1979. Hydrology and Geochemistry of Thermal Springs of the Appalachians. U.S. Geological Survey Professional Paper 1044-E.

Hooper and Angus Associates Ltd. 1979. Study to Examine the Technical and Economic Feasibility of Using Aquifers as a Chilled water Source or Storage Medium for Building Air Conditioning Systems - Vol. I., Toronto, Canada.

Hooper and Angus Associates Ltd. 1979. Study to Examine the Technical and Economic Feasibility of Using Aquifers as a Chilled Water Source or Storage Medium for Building Air Conditioning Systems - Volume II. Toronto, Canada.

Hubbert, M.K. 1940. "The Theory of Ground-Water Motion." Journal of Geology. XLVIII $(8): 765-944$.

Intercomp Resource Development and Engineering, Inc. 1976. A Model for Calculating Effects of Liquid Waste Disposal in Deep Saline Aquifers, Part I -- Development. 76-61. U.S. Geological Survey Water Resources Investigations.

Jacob, C.E. 1950. "Flow of Groundwater." In Engineering Hydraulics, pp.320386. H. Rouse, ed. John Wiley \& Sons, Inc. New York, New York.

Jenne, E.A., ed. 1979. Chemical Modeling in Aqueous Systems. American Chemical Society Symposium Series 93. p. 914.

Joos, B., 1978. "Tests de Sensibilite du Programme Diffusion-Transport." In Rapports Scientifiques et Techniques sur le Comportement des Nappes Souterraines, Vol. 6 . Institute de Production d'Energie, Centre d'Hydrogeologie de l'Universite de Neuchatel, Lusanne, Switzerland.

Katz, D.L. and K.H. Coats. 1973. Underground Storage of Fluids. Ulrich's

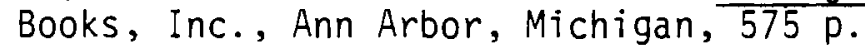

Kazmann, R.G. 1971. "Exotic Uses of Aquifers." Proceedings American Society Civil Engineers. 97(IR3):515-522.

Keenan, J.H., et al. 1969. Steam Tables. John Wiley, New York, New York, p. 162 .

Kharaka, Y.K., and I. Barnes. 1973. SOLMNEQ: Solution-Mineral Equilibrium Calculations. 215-899, Survey Computer Contribution. 
Koch, E., et a1. 1973. Design and Operation of the Artificial-Recharge Plant at Bay Park, New York. U.S. Geological Survey Professional Paper $751-B$.

Kruseman, G.P., and N.A. DeRidder. 1976. Analys is and Evaluation of Pumping Test Data. International Institute for Land Reclamation and Improvement Bulletin 11, Wageningen, The Netherlands.

Lauwerier, H.A. 1955. "The Transport of Heat in an $0 i 1$ Layer Caused by the Injection of a Hot Fluid." Appl. Sci. Res. Ser.A, 5(2-3):145-150.

Leggette, R.M., and M.L. Brashears, Jr. 1938. "Ground-Water for AirConditioning on Long Island, New York." Transactions, American Geophysical Union: 412-418.

Lewis, G.N., and M. Randa11. 1961. Thermodynamics. 2nd ed., rivised by K.S. Pitzer and Leo Brewer, McGraw-Hi11, New York, New York, p. 723.

Lippmann, M.J., and C.F. Tsang. 1980. "Ground Water Use for Cooling: Associated Aquifer Temperature Changes." Ground Water. 18(5):452-458.

List, E.J., and N.H. Brooks. 1967. "Lateral Dispersion in Saturated Porous Media." Journal of Geophysical Research. 72(10):2531-2541.

Maddock, T. III, et al. 1979. Management Model for Electrical Power Production from a Hot-Water Geothermal Reservoir. University of Arizona Technical Report No. 34.

Mangold, D.C., et a1. 1980. CCC User's Manual - Versions II (Draft). LBL10909, Lawrence Berkeley Laboratory, University of California, Berkeley, California.

Marshal1, T., and W.R. Braithwaite. 1973. "Corrosion Control in Geothermal Systems." In Geothermal Energy, UNESCO Earth Science Ser. No.12, p.151-160, H.C.H. Amstead, ed. Paris, France

Mathey, B. 1977. "Development and Resorption of a Thermal Disturbance in a Phreatic Aquifer with Natural Convection." Journal of Hydrology. 34:315-333.

Mathey, B., and A. Menjos. 1978. Underground Heat Storage: Choice of a Geometry and Efficiency. In Proceedings, Thermal Energy Storage in Aquifers Workshop, Lawrence Berkeley Laboratory, LBL-8431, Berkeley, Calif.

Mercer, J.W., and C.R. Faust. 1979a. "Geothermal Reservoir Simulation 3. Application of Liquid- and Vapor-Dominated Hydrothermal Modeling Techniques to Wairakei, New Zealand." Water Resources Research. 15(3): 653-671. 
Mercer, J.W., and C.R. Faust. 1979b. "A Review of Numerical Simulation of Hydrothermal Systems." Hydrological Sciences. Bulletin 24, 3, 9:335-343.

Mercer, J.W., and C.R. Faust. 1980. "Ground-Water Modeling: Mathematical Mode1s." Ground Water. 18(3):212-227.

Meyer, C.A., et al. 1968. ASME Steam Tables. 2nd edition. American Society of Mechanical Engineers, New York, New York. p. 328.

Meyer, C.F., et al. 1972. Thermal Storage for Eco-Energy Utilities. GE72TiMP-56, General Electric Company - TEMPO, Santa Barbara, Calif.

Meyer, C.F., et al. 1976. Role of the Heat Storage Well in Future U.S. Energy Systems. GE76TMP-27A, General Electric - TEMPO, Santa Barbara, Calif.

Meyer, C.F., and D.K. Todd. 1973A. "Conserving Energy with Heat Storage Wells." Environmental Science and Technology. ㄱ(6):512-516.

Meyer, C.F., and D.K. Todd. 1973b. Heat Storage Wells for Conserving Energy and Reducing Thermal Pollution. P-635, General Electric Company TEMPO, Santa Barbara, California.

Molz, F.J., and L.C. Be11. 1977. "Head Gradient Control in Aquifers Used for Fluid Storage." Water Resources Research. 13(4):795-798.

Molz, F.J., et al. 1978. "Aquifer Storage of Heated Water: Part I - A Field Experiment." Ground Water. 16(4):234-241.

Molz, F.J., et al. 1979. "Thermal Energy Storage in a Confined Aquifer: Experimental Results." Water Resources Research. 15(6):1509-1514.

Molz, F.J., et al. 1980. Experimental Study of the Storage of Thermal Energy in Confined Aquifers. ORNL/Sub-7336/1-UC94a. Prepared for Oak Ridge National Laboratory by the Water Resources Research Institute, Auburn University, Auburn, Alabama.

More1, Francois, and J. Morgan. 1972. "A Numerical Method for Computing Equilibria in Aqueous Chemical Systems." Environmental Science and Technology $\underline{6}: 58-57$.

Mungan, N. 1965. "Permeability Reduction Through Changes in pH and Salinity." Journal Petroleum Technology. Dec.:1449-1453.

Narasimhan, T.N., et al. 1978. "Numerical Model for Saturated-Unsaturated Flow in Deformable Porous Media. 2. The Algorithm." Water Resources Research. 14(2):255-261. 
Narasimhan, T.N., and P.A. Witherspoon. 1976. "An Integrated Finite Difference Method for Analyzing Fluid Flow in Porous Media." Water Resources Research. 12(1):57-64.

Nordstrom, D.K., et a1. 1979. "A Comparison of Computerized Chemical Models for Equilibrium Calculations in Aqueous Systems." pp. 857-892. E.A. Jenne, ed. Chemical Modeling in Aqueous Systems: American Chemical Society Symposium Series 93.

Ogata, A., and R. Banks. 1961. A Solution of the Differential Equation of Longitudinal Dispersion in Porous Media. U.S. Geological Survey Professional Paper 411-A, p. 7.

Papadopulos, S.S., and S.P. Larson. 1978. "Aquifer Storage of Heated Water: Part II - Numerical Simulation of Field Results." Ground Water. 16(4): $242-248$.

Parkhurst, D.L. et al. 1980. PHREEQE - A Computer Program for Geochemical Calculations. U.S. Geological Survey Water-Resources Investigations

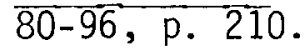

Patton, C.C. 1974. Oilfield Water Systems. 'Campbel1 Petroleum Series, Norman, Oklahoma, p. 178.

Peaceman, D.W. 1977. Fundamentals of Numerical Reservoir Simulation. Elsevier, New York, New York, p. i76.

Pearson, F.J., Jr., et al. 1978. "Correction of Ground-Water Chemistry and Carbon Isotopic Composition for Effect of $\mathrm{CO}_{2}$ Outgassing." Geochim. Cosmochim. Acta. 42:1799-1807.

Pelka, W. 1979. Two Well Storage Systems for Combined Heating and Air Conditioning by Groundwater Heat Pumps in Shallow Aquifers. Lawrence Berkeley Laboratory, University of California, Berkeley, Cal ifornia.

Pinder, G.F. 1979. State-of-the-Art Review of Geothermal Reservoir Modeling. LBL-9093-GSRMP-S-UC-66a, Lawrence Berkeley Laboratory, University of California.

Pinder, G.F., and J.D. Bredehoeft. 1968. "Application of the Digital Computer for Aquifer Evaluation." Water Resources Research. 4(5):1069-1093.

Pinder, G.F., and W.G. Gray. 1977. Finite Element Simulation in Surface and Subsurface Hydrology. Academic Press, New York, New York. p. 295.

Pinder, G.F., et al. 1980. GAFETTA - A Galerkin Assymmetric Finite Element Thermal Transport Aquifer Model. Report 80 08, Department of Water Resources Engineering, Royal Institute of Technology, Stockholm, Sweden. 
Pitzer, K.S. 1973. "Thermodynamics of Electrolytes. I. Theoretical Bas is and General Equations." Journal Physical Chemistry. 77:268-277.

Plummer, L.N. 1975. "Mixing of Sea Water with Calcium Carbonate Ground Water". Geological Society America Memoir 142:219-236.

Plummer, L.N., et al. 1976. WATEQF-A Fortran IV Version of WATEQ, a Computer Program for Calculating Chemical Equilibrium of Natural Water. U.S. Geological Survey Water-Resources Investigations 76-13. (Revised and Reprinted December 1978).

Poreh, M. 1965. "The Dispersivity Tensor in Isotropic and Axisymmetric Mediums." Journal of Geophysics Research. 70(16):3909-3913.

Potter, R.W., II. 1979. Computer Modeling in Low Temperature Geochemistry. Feviews of Geophysics and Space Physics. V. 17, pp. 850-855.

Prickett, T.A., and C.G. Lonnquist. 1971. Selected Digital Computer Techniques for Groundwater Resource Evaluation. Illinois State Water Survey Bu11. 55, Urbana, Illinois.

Prigogine, I., and R. Defay. 1954. Chemical Thermodynamics. Longmans Green, London, England.

Qvale, E.B. 1978. The Danish Seasonal Aquifer Warm-Water Storage Program. In Proceedings, Thermal Energy Storage in Aquifers Workshop. LBL-8431, Lawrence Berkeley Laboratory, Berkeley, Calif.

Rabbimov, R.T., et al. 1971. "Storage of Solar Energy in a Sandy-Gravel Ground." Geoliotekhnika. I(5):57-64.

Ragone, S.E. 1977. Geochemical Effects of Recharging the Magothy Aquifer, Bay Park, New York, with Tertiary-Treated Sewage. U.S. Geological Survey Professional Paper 751-D.

Ragone, S.E., et al. 1975. "Mobilization of Iron in Water in the Magothy Aquifer During Long-Term Recharge with Tertiary-Treated Sewage, Bay Park, New York." Journal Research U.S. Geological Survey. 3:93-98.

Ragone, S.E., and J. Vecchioli. 1975. "Chemical Interaction During Deep Well Recharge, Bay Park, New York." Ground Water. 13.

Ramey, H.J., Jr. 1967. "A Current Review of 0il Recovery by Steam Injection." Proceedings Seventh World Petroleum Cong. 3:471-476.

Redde11, D.L., and K.K. Sunada. 1970. Numerical Simulation of Dispersion in Groundwater Aquifers. Hydrology Papers No. 41, Colorado State Univ., Fort Collins, Colorado. 
Redde11, D.L., et a1. 1978. Thermal Storage of Cold Water in Ground-Water Aquifers for Cooling Purposes. LBL-8431, Thermal Energy Storage in Aquifers Workshop Proceedings. Lawrence Berkeley Laboratory, Berkeley, Calif.

Reed, M.G. 1977. "Formation Permeability Damage by Mica Alteration and Carbonate Dissolution." Journal Petroleum Technology. Sept.:1956-1960.

Reffstrup, J. 1979. Prediction of Recovery Rations and Flow Conditions in Connection with Seasonal Thermal Energy Storage in Confined Aquifers. Laboratory for Energetics, Lyngby, Denmark, Ph.D. Thesis, Technical University of Denmark.

Reilly, P.J., et al. 1971. "The Prediction of Osmotic and Activity Coefficients in Mixed-Electrolyte Solutions." Journal Physical Chemistry. 75: $1305-1315$.

Reilly, R.W. 1980. A Descriptive Analysis of Aquifer Thermal Energy Storage Systems. PNL-3298, Pacific Northwest Laboratory, Battel Te Memorial Institute, Richland, Washington.

Remson, I., et al. 1971. Numerical Methods in Subsurface Hydrology. Wiley-Interscience, New York, New York, p. 389.

Rimstidt, J.D., and H.L. Barnes. 1980. "The Kinetics of Silica-Water Reactions." Geochim. Cosmochim. Acta. 44:1683-1699.

Rubinstein, L.I. 1972. Thermal Effect in $0 i 1$ Reservoirs. Moscow:Nedra, Chap. V-VII.

Sauty, J.P., et al. 1978. The Effect of Thermal Dispersion on Injection of Hot Water in Aquifers. LBL-8883, Lawrence Berkeley Laboratory, Univ. of California Second Invitational Well-Testing Symposium Proceedings; Berkeley, Calif.

Sauty, J.P. 1980. "An Analysis of Hydrodispersive Trarsfer in Aquifers." Water Resources Research. 16(1):145-158.

Sauty, J.P., et al. 1981. "Sensible Energy Storage in Aquifers, 1 Theoretical Study." Submitted to Water Resources Research.

Schaetzle, W.J., et a1. 1980. Thermal Energy Storage in Aquifers: Design and Application. Pergamon Press, Inc., New York, New York, p. 177.

Scheidegger, A.E. 1961. "General Theory of Dispersion in Porous Media." Journal Geophysical Research. 66 (10):3273-3278.

Somerton, W.H., et al. 1974. "Thermal Behavior of Unconsolidated 0il Sands." Society Petroleum Engineering Journal. Oct.:513-521. 
Stottlemyre, J.A. et al. 1979. Geocherlical Equilibrium Modeling of the Auburn Thermal Energy Storage Field Test. PNL-3167, Pacific Northwest Laboratory, Battelle Memorial Institute, Richland, WA.

Stottlemyre, J.A. et al. 1980. Physicochemical Properties Analysis in Support of the Seasonal Thermal Energy Storage Program. Proceedings of Mechanica T, Magnetic and Underground Energy Storage 1980 Annua 1 Contractors' Review, CONF-801128, Nov. 10-13.

Streltsova-Adams, T.D. 1978. Fluid Flow in Naturally Fractured Reservoirs. Second Invitational well-Testing Symposium Proceedings, 0ct. 25-27, Berkeley, California.

Subcasky, W.J. 1978. Petroleum Industry Experience in Water Injection. In Proceedings, Thermat Energy Storage in Aquifers Workshop, LBL-8431, UC-94a, CONF-7805140.

Terzaghi, K. 1925. Principles of Soil Mechanics, A Summary of Experimental Results of Clay and Sand. Engineering News Record, 3-98.

Thomas, R.G. 1973. Groundwater mode1s. Irrigation and Drainage Paper 21. Food and Agriculture Organization of the United Nations, Rome, Italy, p. 192.

Thorstenson, D.C., et a1. "The Geochemistry of the Fox Hi11s - Basal Hel1 Creek Aquifer in Southwestern North Dakota and Northwestern South Dakota." Water Resources Research. 15:1479-1498.

Truesde11, A.H., and B.F. Jones. 1974. "WATEQ, A Computer Program for Calculating Chemical Equilibria of Natural Waters." Journal Research U.S. Geological Survey, 2:233-248.

Truesdell, A.H., and W. Singers. 1974. "The Calculation of Aquifer Chemistry in Hot-Water Geothermal Systems." Journal Research U.S. Geological Survey, 2:271-278.

Tsang, C.F. 1979. A Review of Current Aquifer Thermal Energy Storage Projects. Lawrence Berkeley Laboratory, University of California,

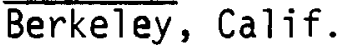

Tsang, C.F. 1980b. Aquifer Thermal Energy Storage Studies at LBL in 1980. Proceedings of Mechanical, Magnetic and Underground Energy Storage 1980 Annual Contractors' Review, CONF-801128, Nov. 10-13.

Tsang, C.F., et al. 1976. Numerical modeling of Cyclic Storage of Hot Water in Aquifers. Presented at the Fall Annual Meeting of the American Geophysical Union, Dec. 6-10, San Francisco, Calif.

Tsang, C.F., et a1. 1977. "Production and Reinjection in Geothermal Reservoirs." Geothermal Resource Council Trans. 1:301-303. 
Tsang, C.F., et al. 1978a. Mathematical Modeling of Thermal Energy Storage in Aquifers. LBL-8431, Lawrence Berkeley Laboratory, University of

California. Presented at the Thermal Energy Storage in Aquifers Workshop, Berkeley, Calif.

Tsang, et al. 1978b. Underground Aquifer Storage of Hot: Water From Solar Energy Collectors. Presented at the International Solar Energy Congress, New Delhi, India.

Tsang, C.F. et al. 1980a. Aquifer Thermal Energy Storage - A Survey. Lawrence Berkeley Laboratory, University of California., Berkeley, Calif.

Tsang, C.F., et al. 1980b. Experimental and Theoretical Studies of Thermal Energy Storage in Aquifers. Presented at the 15th Intersociety Energy Conversion. Engineering Conference, Seattle, Washington.

Tyson, H.N., and E.M. Weber. 1964. "Groundwater Management for the Nations Future - Computer Simulation of Groundwater Basins." lournal Hydraul. Div. Amer. Soc. Civil Eng. 90(HY4):59-77.

Van Zeggeren, F., and S.H. Storey. 1970. The Computation of Chemical Equilibria. Cambridge University Press, p. 176.

Vecchioli, J. 1970. "A Note on Bacterial Growth Around a Recharge Well at Bay Park, Long Island, New York." Water Resources Fiesearch. 6:14151419 .

Vecchioli, John, et al. 1974. Geohydrology of the Artificial-Recharge Site at Bay Park, Long Island, New York. U.S. Geol. Survey Professional Paper 751-C.

Vecchioli, John, and H.F.H. Ku. 1972. Preliminary Results of Injecting Highly Treated Sewage-Plant Effluent into a Deep Sand fquifer at Bay Park, New York. U.S. Geological Survey Professional Paper 751-A.

Verruijt, A. 1970. Theory of Groundwater Flow. Gordon and Breach Science Publishers, New York, New York, p. 190.

Voss, C. 1980. Notes from an International Seminar on reat Storage in Aquifers, Apri1 15-16, 1980. Lund-MPh-80/13, Lund Inst itute of Technology, Lund, Sweden.

Voss, C. et al. 1981. Simulation of Low Temperature Seasonal Energy Storage in a Shallow Glaciofluvial Aquifer. Report 8011, Swedish Board for Energy Source Development, Royal Institute of Technology, Stockholm, Sweden.

Walton, W.C. 1970. Groundwater Resource Evaluation. McGraw-Hill Book Co., New York, New York, p. 664. 
Warner, D.L. and J.H. Lehr. 1977. An Introduction to the Technology of Subsurface Waste Water Injection. Environmental Protection Technology Series EPA-600/2-77-240.

Werner, D., and W. Kley. 1977. "Problems of Heat Storage in Aquifers." Journal of Hydrology $34: 35-43$.

Wigley, T.M.L. 1977. WATSPEC: A Computer Program for Determining the Equilibrium Speciation of Aqueous Solutions. British Geomorphological Research Group Technical Bulletin No. 20.

Willhite, G.P., et al. 1974. Disposal of Heated Water Through GroundWater Systems: Volume 1 - Technical and Economic Feasibility. The Kansas Water Resources Research Institute, University of Kansas.

Wolery, T.J. 1979. Calculation of Chemical Equilibrium Between Aqueous Solution and Minera7s: The EQ3/6 Software Package. UCRL-52658, Lawrence Livermore Laboratory, Livermore, Calif.

Wolery, T.J., and L.G. Walters, Jr. 1975. "Calculation of Equilibrium Distributions of Chemical Species in Aqueous Solutions by Means of Monotone Sequences." Mathematical Geology. 7:99-115.

Yokoyama, T., et al. 1978. Seasonal Regeneration Through Underground Strata. LBL-8431, Thermal Energy Storage in Aquifers Workshop Proceedings, Lawrence Berkeley Laboratory, Berkeley, Calif.

Yokoyama, T., et al. 1980. "Seasonal Thermal Storage in Aquifer for Utilization." Bulletin of the JSME. 23(184):1646-1654.

Zienkiewicz, 0.C. 1971. The Finite Element Method in Engineering Science. McGraw-Hi11, London, England, p. 521. 


\section{APPENDIX A. EQUATION DEVELOPMENT}

The governing flow and transport equations describing aquifer thermal energy storage (ATES) may be obtained by several different methods. A rigorous formulation of the governing equations is through formal integration of the microscopic or pore-level balance equations over the porous medium (see, for example, Faust and Mercer 1977). Alternatively, a somewhat less rigorous approach is used in this appendix. It begins with balances for mass, momentum and energy. Before presenting these balance equations, however, a discussion of viewpoints is presented. 


\section{VIEWPOINTS}

Assuming we can identify or in some way understand the concept of ATES and related processes of interest, the development of a mathematical model may be approached from (A. Klute, written comm. 1970): (1) the molecular, (2) the microscopic or (3) the macroscopic viewpoint. A discussion of these viewpoints is important since confusion often arises between hydrologists over which viewpoint they are considering. An example of this is a modeler discussing chemical source terms at the macroscopic leve: with a geochemist who is describing the same chemical source terms at the molecular level.

In the molecular point of view, theories and explanations of the mechanisms of flow are given in terms of the behavior of the water molecules. For this approach, statistical mechanical concepts might be used. Chemical reaction mechanisms are generally described using this viewpoint.

At an intermediate level, the microscopic, a theory of flow may be developed treating water in the pores as a continuum and applying the principles of continuum mechanics, especially fluid mechanics, to work out the detailed behavior of the water within the pores. An example of this approach is using the Navier-Stokes equation for the flow of a viscous fluid to work out the detailed water velocity pattern within the pores. However, in natural environments, the identification of pore geometry and other conditions necessary for the solution of the equations is virtually impossible and thus solutions are generally available only for rather simple pore space geometry, such as flow in straight capillary tubes, or between parallel plates. This approach may be used to describe flow in fractures assuming simple geometry.

From a practical viewpoint, one generally must continue the theoretical development to the macroscopic level in order to create a useful tool. We cannot observe the behavior of individual molecules, nor can we observe the velocity and fluid pressure distributions that one might, in principle, calculate in the microscopic approach. All measurements in the field are generally made at the macroscopic level; therefore, to be useful, any theory of water movement must be developed to the point of describing flow on the macroscopic level. 
In the macroscopic approach, all variables are assumed continuous functions of space and time, with derivatives of as high an order as needed. The permeable medium is treated as a superposition of two continuous phases, solid and liquid. Velocities, hydraulic heads and other necessary variables are treated as point functions. That is, they are defined at every point in the region of interest. The way these variables are defined is important since it involves choosing a volume element of the permeable medium that is representative of the medium. For example, consider the bulk density, or mass of solids per unit bulk volume of permeable medium, at a point surrounded by some volume element. From Figure $A-1$, we see that if we choose the volume element smaller than $V_{1}$, then we may either have a volume of all solid or a volume of all liquid, causing the value for bulk density to vary. Alternatively, if we choose a volume element larger than $V_{2}$, the value of bulk density again varies due to heterogeneous effects (i.e., major changes in rock type). In practice the volume element (which in some literature is referred to as an REV or Representative Elementary Volume) is taken large enough to contain a representative assortment of pores, but at the same time small enough so that the values of macroscopic variables are approximately constant within the element. In our figure, this volume lies between $V_{1}$ and $V_{2}$. In the following development, all equations and coefficients are considered at the macroscopic level.

\section{MOMENTUM BALANCE}

For the momentum balance, Darcy's equation, which is based on empirical evidence, is used. In genera1, Darcy's equation relates a rate of flow, q, (volume per unit time per unit cross-sectional area normal to the direction of flow) to an energy loss gradient, $\Delta E / L$, by a proportionality constant, $K^{\prime}$ :

$$
q=K^{\prime} \Delta E / L
$$

where $E$ is the total potential energy and $L$ is the length over which the difference $\Delta E$ is applied. In ground-water problems, the potential energy is generally expressed in one of three ways: (1) pressure, $p$, (2) hydraulic potential, $\Phi$, or (3) hydraulic head, h. 


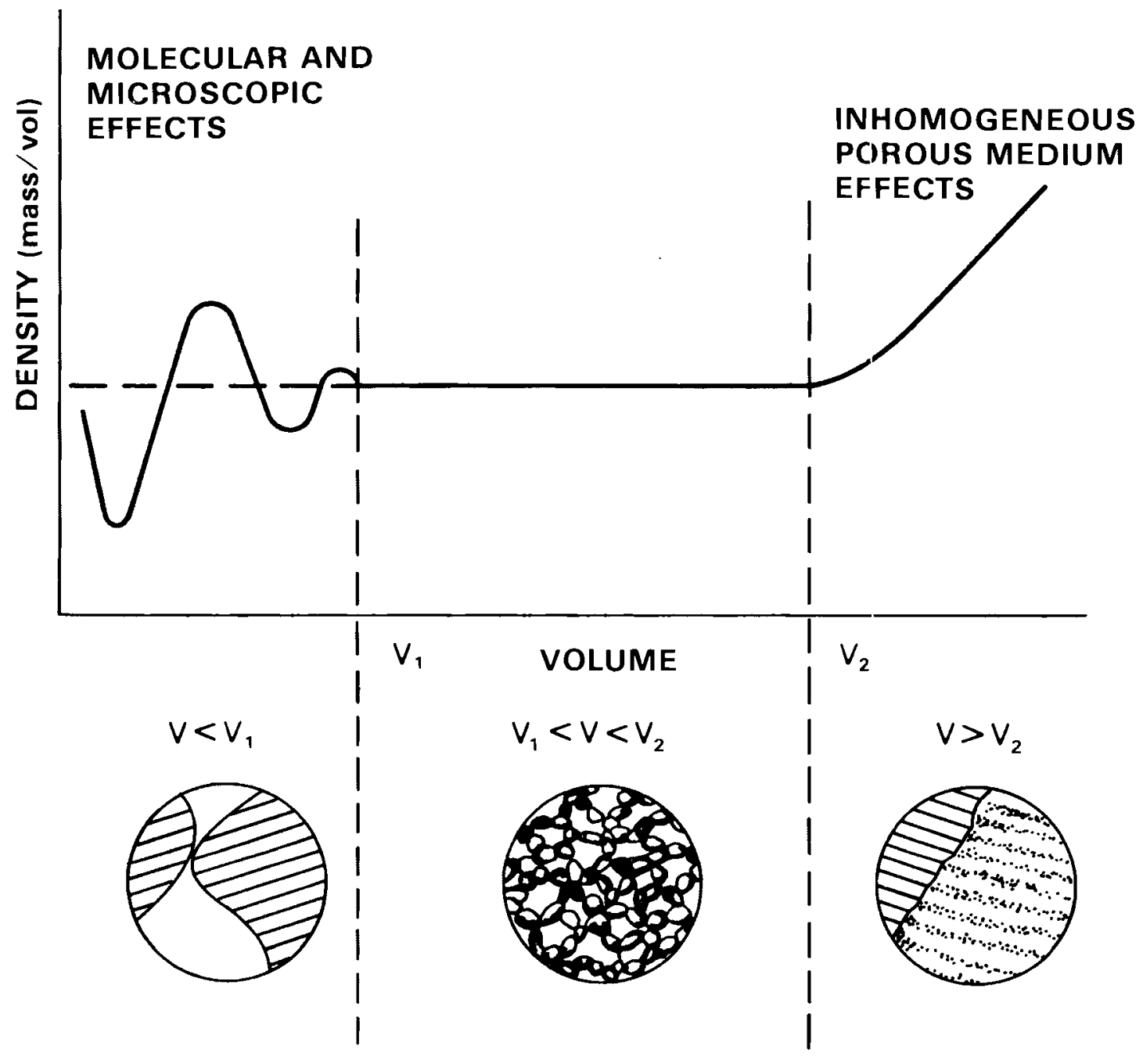

FIGURE A-1. Variation In Bulk Density As $A$ Function of The Average Volume, With Inserts

(from Mercer and Faust 1980) 
Let $V$ be a volume element, $\rho$ be density and $g$ be the gravitational acceleration constant, then $\rho V$ is the mass of the element and $\rho g V$ is the weight of the element. Pressure (force per unit area or energy per unit volume) is defined on a per unit volume basis as $\mathrm{p}=\mathrm{E} / \mathrm{V}$; hydraulic potential is defined on a mass basis as $\Phi=\mathrm{E} / \rho \mathrm{V}$; and hydraulic head is defined on a weight basis as $h=E / \rho g V$. Note that both hydraulic potential and hydraulic head are density dependent; therefore, for problems that involve variable density, hydraulic potential and hydraulic head are nonconservative (that is, they are path-dependent). For variable density problems, such as those associated with ATES, the pressure formulation is more accurate.

Darcy's equation may be written in terms of pressure as:

$$
\bar{q}=-\frac{\bar{k}}{\mu} \cdot(\bar{\nabla} p-\rho g \bar{\nabla} D)
$$

where

$$
\begin{aligned}
\bar{q} & =\text { flux, volumetric flow rate per unit area, }\left(\mathrm{Lt}^{-1}\right) ; \\
\overline{\bar{k}} & =\text { local intrinsic permeability tensor, }\left(\mathrm{L}^{2}\right) ; \\
\mu & =\text { dynamic viscosity of fluid, }\left(M L^{-1} \mathrm{t}^{-1}\right) ; \\
\mathrm{p} & =\text { pressure, }\left(\mathrm{ML}^{-1} \mathrm{t}^{-2}\right) ; \\
\rho & =\text { fluid density, }\left(M L^{-3}\right) ; \\
g & =\text { gravitational constant, }\left(\mathrm{Lt}^{-2}\right) ; \\
\bar{\nabla} \mathrm{D} & =\text { unit vector associated with gravity (positive downward), (L); and } \\
\bar{\nabla} & \text { is the differential operator defined as: } \\
\bar{\nabla} & =\operatorname{grad}=\overline{\mathrm{i}} \frac{\partial}{\partial \mathrm{x}}+\overline{\mathrm{j}} \frac{\partial}{\partial y}+\bar{k} \frac{\partial}{\partial z}
\end{aligned}
$$

where $\bar{i}, \bar{j}$ and $\bar{k}$ are vectors of unit length in the positive $x-, y-$ and $z-$ directions.

\section{MASS BALANCE}

The mass balance is determined by considering changes in the mass of a small volume element of porous material over a small time interval $(\Delta t)$. Figure A-2 shows such a volume element with a source-sink term and flow 


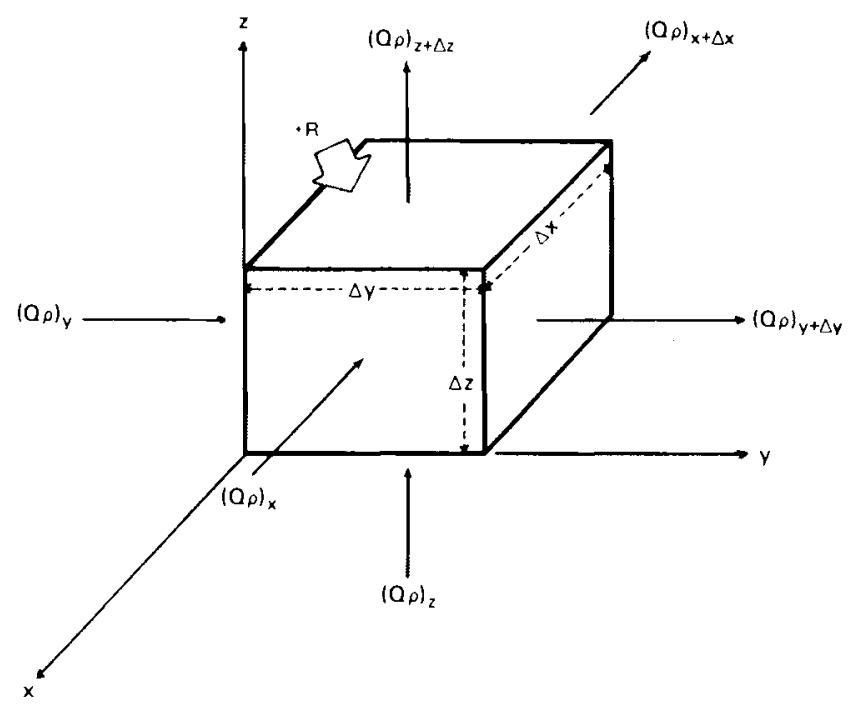

FIGURE A-2. Volume Element of A Porous: Material Showing Flow Across All Faces

(from Mercer and Faust 1980) 
across all faces. In words, the mass balance equation is

(mass leaving - mass entering) $+($ final mass - initial mass $)=0 .(A-4)$

Using the quantities shown in Figure $A-2$, Equation (A-4) may be written to give

$$
\begin{aligned}
& \left\{\left[(Q \rho)_{x+\Delta x}+(Q \rho)_{y+\Delta y}+(Q \rho)_{z+\Delta z}\right]\right. \\
& \left.-\left[(Q \rho)_{x}+(Q \rho)_{y}+(Q \rho)_{z}+\rho R \Delta x \Delta y \Delta z\right]\right\} \Delta t \\
& +\left[(\phi \rho)_{t+\Delta t}-(\phi \rho)_{t}\right] \Delta x \Delta y \Delta z=0
\end{aligned}
$$

where

$$
\begin{aligned}
& Q=\text { rate of volume flow, }\left(\mathrm{L}^{3} \mathrm{t}^{-1}\right) ; \\
& R=\text { volumetric injection rate per unit volume, }\left(\mathrm{t}^{-1}\right) ; \text { and } \\
& \phi=\text { porosity (dimensionless). }
\end{aligned}
$$

Dividing Equation $(A-5)$ by $\Delta x \Delta y \Delta z \Delta t$ and rearranging terms gives

$$
\begin{aligned}
& \frac{\left[(Q \rho)_{x+\Delta x}-(Q \rho)_{x}\right]}{\Delta x \Delta y \Delta z}-\frac{\left[\left(Q_{\rho}\right)_{y+\Delta y}-(Q \rho)_{y}\right]}{\Delta x \Delta y \Delta z}-\frac{\left[(Q \rho)_{z+\Delta z}-(Q \rho)_{z}\right]}{\Delta x \Delta y \Delta z} \\
& \quad+\rho R=\frac{\left[(\phi \rho)_{t+\Delta t}-(\phi \rho)_{t}\right]}{\Delta t} .
\end{aligned}
$$

The Darcy velocity is the flow volume across the element face divided by the area of the face, or

$$
q_{x}=\frac{Q_{x}}{\Delta y \Delta z} ; \quad q_{y}=\frac{Q_{y}}{\Delta x \Delta z} ; \quad q_{z}=\frac{Q_{z}}{\Delta x \Delta y} .
$$


Using Equations ( $A-7)$, and choosing smaller and smaller values of $\Delta x, \Delta y$, $\Delta z$ and $\Delta t$, and using the definition of a derivative, Equation (A-6) becomes

$$
-\frac{\partial\left(\rho q_{x}\right)}{\partial x}-\frac{\partial\left(\rho q_{y}\right)}{\partial y}-\frac{\partial\left(\rho q_{z}\right)}{\partial z}+\rho R=\frac{\partial(\phi \rho)}{\partial t}
$$

or

$$
-\bar{\nabla} \cdot(\rho \bar{q})+\rho R=\frac{\partial(\phi \rho)}{\partial t} \text {. }
$$

Substitution of Equation (A-2) for $\bar{q}$ gives

$$
\bar{\nabla} \cdot\left[\frac{\bar{k} \rho}{\mu} \cdot(\bar{\nabla} p-\rho g \bar{\nabla} D)\right]+\rho R=\frac{\partial(\phi \rho)}{\partial t}
$$

which is the unsteady or transient, three-dimensional ground-water flow equation.

\section{ENERGY BALANCE}

The energy balance may be determined by using the same approach as was taken in developing the mass balance. The conservation of energy principle requires that, over a time interval $(\Delta t)$,

(energy leaving - energy entering $)+($ final energy - initial energy $)=0$.

Using the same volume element in Figure A-2, but now considering energy, the components in Equation (A-11) are:

(1) Energy transport by convection, that is, energy transferred into the volume element with the fluid.

(2) Energy transport by conduction, which is energy transferred across the boundaries of the volume element due to the existence of temperature gradients across the boundaries. This will occur in both the fluid and the solid matrix of a porous medium.

(3) Energy transport by dispersion in the fluid, that is, energy transferred into the volume element associated with microscopic variations in velocities within individual pores.

(4) Energy transport associated with a source/sink term. 
Using these components, Equation (A-11) may be written as

$$
\begin{aligned}
& \left\{\left[\left(Q \rho c_{p} T\right)_{x+\Delta x}+\left(Q \rho c_{p} T\right)_{y+\Delta y}+\left(Q \rho c_{p} T\right)_{z+\Delta z}\right]\right. \\
& \left.-\left[\left(Q \rho c_{p} T\right)_{x}+\left(Q \rho c_{p} T\right)_{y}+\left(Q \rho c_{p} T\right)_{z}\right]\right\} \Delta t \\
& +\left\{\left[\left(\Lambda_{\mathrm{cw}}+\Lambda_{\mathrm{dw}}+\Lambda_{\mathrm{cr}}\right)_{\mathrm{x}+\Delta \mathrm{x}}+\left(\Lambda_{\mathrm{cw}}+\Lambda_{\mathrm{dw}}+\Lambda_{\mathrm{cr}}\right)_{\mathrm{y}+\Delta \mathrm{y}}\right.\right. \\
& \left.+\left(\Lambda_{\mathrm{cW}}+\Lambda_{\mathrm{dW}}+\Lambda_{\mathrm{cr}}\right)_{\mathrm{z}+\Delta \mathrm{z}}\right] \\
& -\left[\left(\Lambda_{\mathrm{cw}}+\Lambda_{\mathrm{dw}}+\Lambda_{\mathrm{cr}}\right)_{\mathrm{x}}+\left(\Lambda_{\mathrm{cw}}+\Lambda_{\mathrm{dw}}+\Lambda_{\mathrm{cr}}\right)_{\mathrm{y}}\right. \\
& \left.\left.+\left(\Lambda_{\mathrm{cw}}+\Lambda_{\mathrm{dw}}+\Lambda_{\mathrm{cr}}\right)_{\mathrm{z}}\right]\right\} \Delta \mathrm{t}-\rho \mathrm{Rc}_{\mathrm{p}} T^{\prime} \Delta x \Delta \mathrm{y} \Delta \mathrm{z} \Delta \mathrm{t} \\
& +\left\{\left(\phi \rho c_{p}{ }^{\top}\right)_{t+\Delta t}+\left[(1-\phi) \rho_{r} c_{p r}{ }^{\top}\right]_{t+\Delta t}\right. \\
& \left.-\left(\phi \rho c_{p}{ }^{\top}\right)_{t}-\left[(1-\phi) \rho_{r} c_{p r}{ }^{\top}\right] t\right\} \Delta x \Delta y \Delta z=0
\end{aligned}
$$

where the new terms are

$c_{p}=$ heat capacity at constant pressure, per unit mass, $\left(L^{2} t^{-2} T^{-1}\right)$;

$T=$ temperature, $(T)$;

$\Lambda_{C}=$ heat conduction flux, $\left(\mathrm{ML}^{2} \mathrm{t}^{-3}\right)$; and

$\Lambda_{d}=$ dispersive (thermal mixing) flux, $\left(\mathrm{ML}^{2} \mathrm{t}^{-3}\right)$.

In Equation ( $A-12)$, the subscripts $w$ and $r$ refer to water and rock, respectively, and the superscript ' refers to the source/sink term. Implicit in Equation (A-12) are the following assumptions:

(1) Thermal equilibrium exists between the water and the rock.

(2) The reversible internal energy increase in the volume element due to compression is negligible compared to internal energy increase due to convection or conduction (Reffstrup 1979).

(3) Viscous dissipation may be neglected (Reffstrup 1979). 
Dividing Equation ( $A-12)$ by $x$ y $z \mathrm{t}$ and rearranging terms gives

$$
\begin{aligned}
& -\frac{\left[\left(Q \rho c_{p} T\right)_{x+\Delta x}-\left(Q \rho c_{p} T\right)_{x}\right]}{\Delta x \Delta y \Delta z}-\frac{\left[\left(Q \rho c_{p} T\right)_{y+\Delta y}-\left(Q \rho c_{p} T\right)_{y}\right]}{\Delta x \Delta y \Delta z} \\
& -\frac{\left[\left(Q \rho c_{p} T\right)_{z+\Delta z}-\left(Q \rho c_{p} T\right)_{z}\right]}{\Delta x \Delta y \Delta z} \\
& -\frac{\left[\left(\Lambda_{c w}+\Lambda_{d w}+\Lambda_{c r}\right)_{x+\Delta x}-\left(\Lambda_{c w}+\Lambda_{d w}+\Lambda_{c r}\right)_{x}\right]}{\Delta x \Delta y \Delta z} \\
& -\frac{\left[\left(\Lambda_{c w}+\Lambda_{d w}+\Lambda_{c r}\right)_{y+\Delta y}-\left(\Lambda_{c w}+\Lambda_{d w}+\Lambda_{c r}\right)_{y}\right]}{\Delta x \Delta y \Delta z} \\
& -\frac{\left[\left(\Lambda_{c w}+\Lambda_{d w}+\Lambda_{c r}\right)_{z+\Delta z}-\left(\Lambda_{c w}+\Lambda_{d w}+\Lambda_{c r}\right)_{z}\right]}{\Delta x \Delta y \Delta z} \\
& +\rho R c_{p} T^{\prime}=\frac{\left\{\left[\phi \rho c_{p}+(1-\phi) \rho_{r} c_{p r}\right] T\right\}_{t+\Delta t}-\left\{\left[\phi \rho c_{p}+(1-\phi) \rho_{r} c_{p r}\right]\right\}_{t}}{\Delta t}
\end{aligned}
$$

The conductive and dispersive fluxes across the element face may be divided by the area of the face and are given the symbol $\lambda$. Noting this and using Equation (A-7), as with the mass balance, we may choose smaller and smaller values of $\Delta x, \Delta y, \Delta z$ and $\Delta t$. Using the definition of a derivative, Equation (A-13) becomes

$$
\begin{aligned}
& -\frac{\partial\left(\rho q_{x} c_{p} T\right)}{\partial x}-\frac{\partial\left(\rho q_{y} c_{p} T\right)}{\partial y}-\frac{\partial\left(\rho q_{z} c_{p} T\right)}{\partial z} \\
& -\frac{\partial\left(\lambda_{c w}+\lambda_{d w}+\lambda_{c r}\right)_{x}}{\partial x}-\frac{\partial\left(\lambda_{c w}+\lambda_{d w}+\lambda_{c r}\right)_{y}}{\partial y} \\
& -\frac{\partial\left(\lambda_{c w}+\lambda_{d w}+\lambda_{c r}\right)_{z}}{\partial z}+\rho R c_{p} T^{\prime} \\
& =\frac{\partial}{\partial t}\left[\phi \rho c_{p}+(1-\phi) \rho_{r} c_{p r}\right] T
\end{aligned}
$$


or

$$
\begin{aligned}
& -\bar{\nabla} \cdot\left(\rho q c_{p} T\right)-\bar{\nabla} \bar{\lambda}_{c w}-\bar{\nabla} \bar{\lambda}_{d w}-\bar{\nabla} \bar{\lambda}_{c r}+\rho R_{p}{ }^{\prime} \\
& =\frac{\partial}{\partial t}\left[\phi \rho c_{p}+(1-\phi) \rho_{r} c_{p r}\right] \mathrm{T} .
\end{aligned}
$$

It is assumed that the conduction-dispersion terms may be lumped together and are defined by a Fourier-type equation:

$$
\bar{\lambda}_{c w}+\bar{\lambda}_{d w}+\bar{\lambda}_{c r}=-\bar{K}_{m}^{\star} \cdot \bar{\nabla} T
$$

where $\bar{K}_{m}^{*}$ is the medium conduction-dispersion tensor. This tensor is discussed in the main text. Substitution of Equation (A-16) into Equation (A-15) gives

$$
-\bar{\nabla} \cdot\left(\rho \bar{q} c_{p} T\right)+\bar{\nabla} \cdot\left(\bar{K}_{m}^{*} \cdot \bar{\nabla} T\right)+\rho R c_{p} T^{\prime}=\frac{\partial}{\partial t}\left[\phi \rho c_{p}+(1-\phi) \rho_{r} c_{p r}\right] T(A-17)
$$

which is the transient, three-dimensional heat transport equation for water and rock. 

APPENDIX B. NUMERICAL APPROXIMATIONS

\section{FINITE-DIFFERENCE METHODS (FDM)}

One numerical approach that has been applied successfully to ATES involves finite-difference approximations. When using FDM to solve a partial differential equation, a grid is first established throughout the region of interest. For two-dimensional, areal problems, we overlay a grid system on a map view of the aquifer. There are two common types of grids: mesh-centered and block-centered. These are shown in Figure B-1. Associated with the grids are node points that represent the position at which the solution of the unknown values (pressure, for example) is obtained. In the mesh-centered grid the nodes are located on the intersection of grid lines, whereas in the block-centered grid the nodes are centered between grid lines. The choice of the type of grid to use depends largely on the boundary conditions. The mesh-centered grid is convenient for problems where values of pressure are specified on the boundary, whereas the block-centered grid has an advantage in problems where the flux is specified across the boundary. From a practical point of view, the differences in the two types of grids are minor.

Note that the grids shown in Figure $B-1$ are rectangular and regular, that is, the spacing in the $x$-direction, $\Delta x$, and $y$-direction, $\Delta y$, are constant. Often, irregular, rectangular grids with variable $\Delta x$ and $\Delta y$ are used to describe the aquifer, using smaller spacing for areas where more detail is required (as near a well field). Such a grid is shown in Figure B-2. In addition, grids need not be rectangular.

The integrated finite-difference method (IFDM) utilizes an arbitrary grid. Though less commonly applied than the conventional finite-difference approach, the IFDM has been used to study ground-water problems since the early 1960's, when it was known by a different name. Tyson and weber (1964) introduced the method to the ground-water field, but referred to it as a polygonal model technique. The method was further discussed by Cooley (1971) and Thomas (1973), and was finally given the current name by Narasimhan and Witherspoon (1976). 


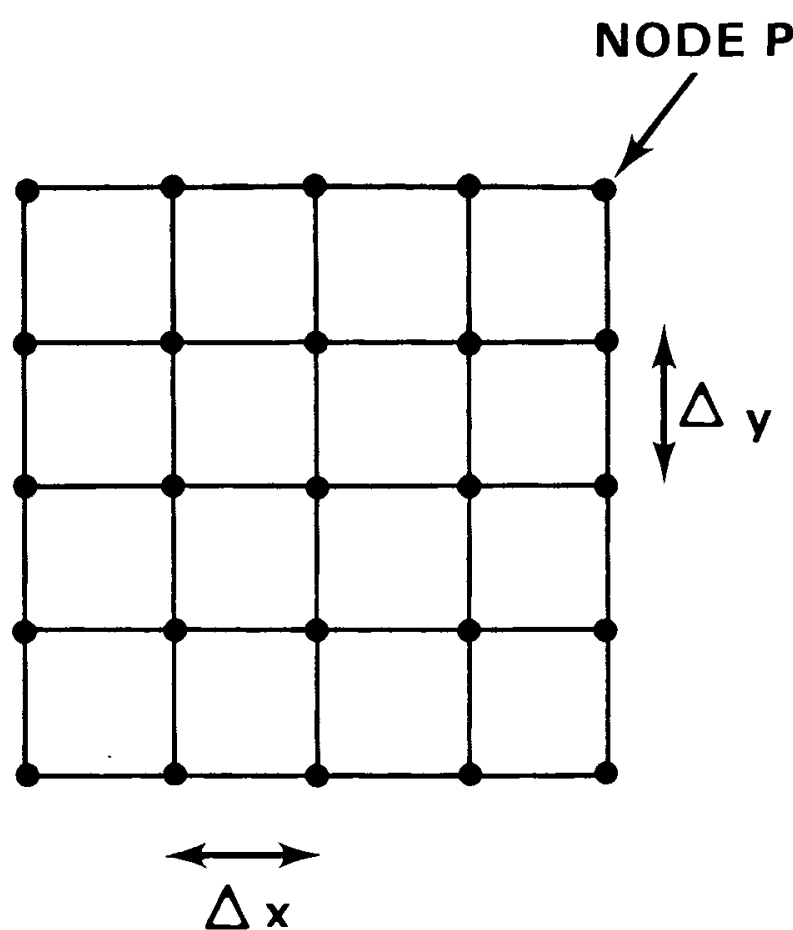

MESH CENTERED

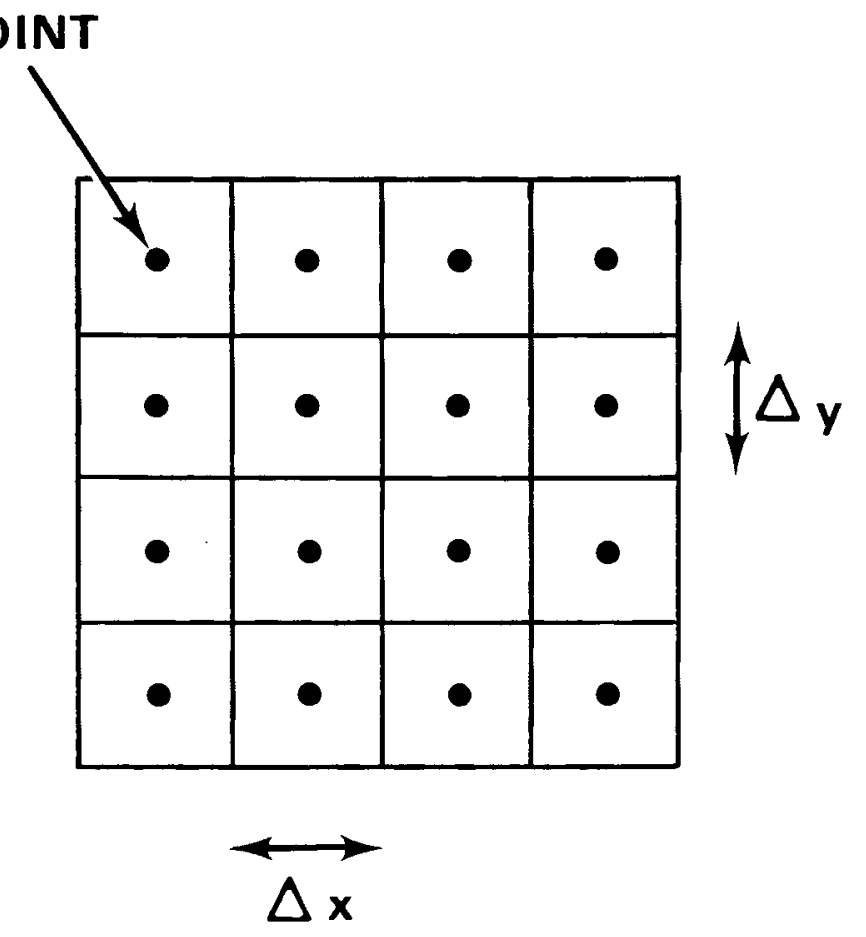

BLOCK CENTERED

FIGURRE B-1. Grids Showing Mesh-Centered

Nodes and Block-Centered Nodes

(from Faust and Mercer 1980) 

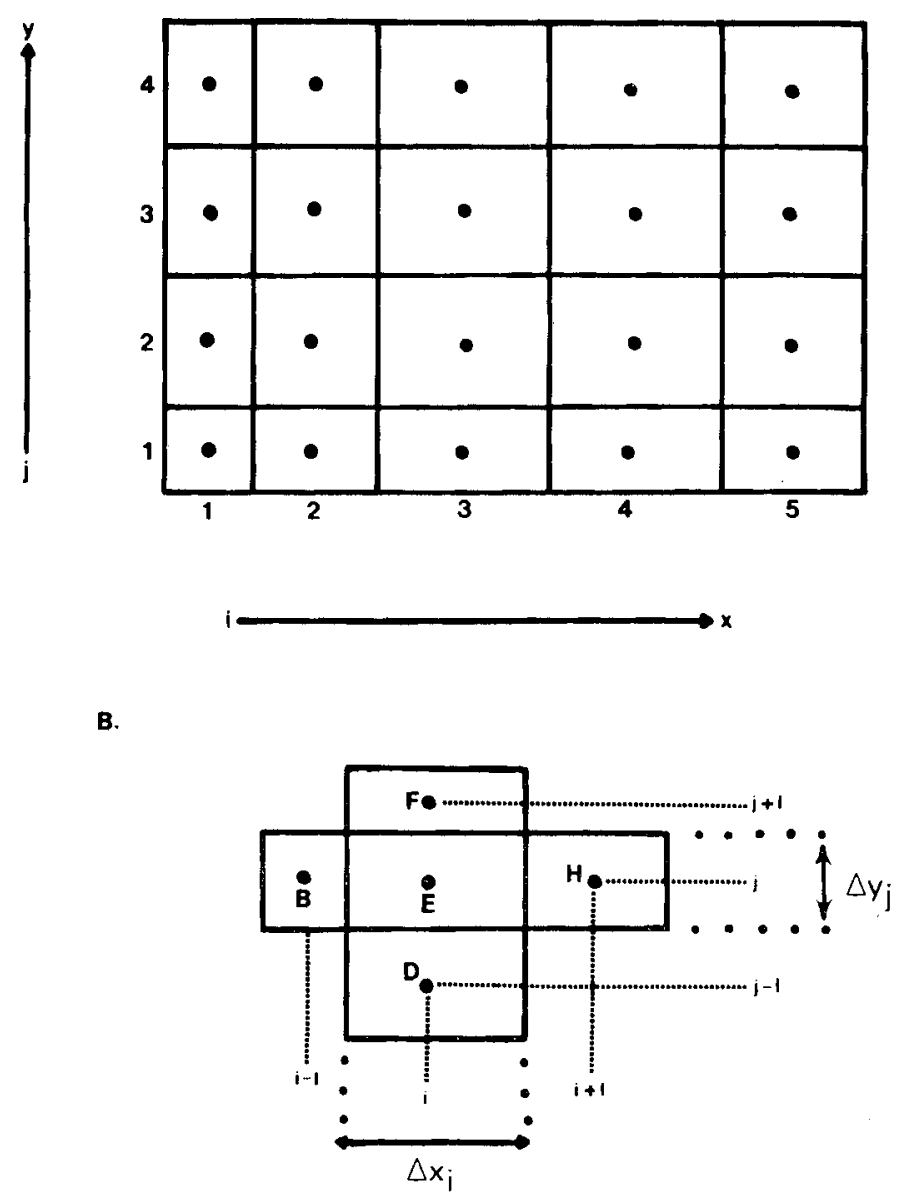

FIGURE B-2. Four by Five Block-Centered Grid Used in Difference Equation Development $(A)$ and Typical Connection (B) for Node $(i, j)$ (from Faust and Mercer 1980) 
For the IFDM, the region of interest must also be divided into smaller areas. Thomas (1973) refers to these as nodal areas, since they each have a node point which is used for mathematical purposes to connect each area with its neighbor. Further, as with other finite-difference methods, it is assumed that all recharge or withdrawal to and from the nodal area occurs at the node point and that water levels in the entire nodal area are the same as at the node point. For this reason, the polygon geometry as well as rectangular grids should be kept to a reasonable size to maintain accuracy.

A typical node point, adjacent nodes, and the polygonal zone associated with it are shown in Figure B-3. As may be seen, the triangles formed by connecting the node points have no interior angle greater than $90^{\circ}$ since the polygon sides are perpendicular bisectors of the lines connecting the intersects. When rectangular nodes are used, the interconnects form rectangles. Thomas (1973) points out that construction errors of more than 5 to 8 degrees from the $90^{\circ}$ limit will result in significant computational errors which cannot be easily identified in the results.

Difference approximations may be developed using truncated Taylor series (that is, only the first few terms in the series are included). Taylor series are used frequently in ground-water hydrology for many purposes. For example, if water density is a weakly dependent function of temperature, it may be expanded about some reference density using a Taylor series. Usually the series is truncated after the first derivative (i.e., truncated in first-order terms), resulting in a linear relationship between density and temperature. For numerical methods, truncated Taylor series may be used to approximate the derivatives in partial differential equations. Alternatively, a more intuitive approach can be used to obtain the final equations by considering the fluxes into and out of a finite-difference block. This second approach is essentially that used to derive the equations for the IFDM. Prickett and Lonnquist (1971) develop finite-difference equations using the second approach and a mesh-centered grid; whereas Freeze and Cherry (1979) present a development based on the first approach and a blockcentered grid. 


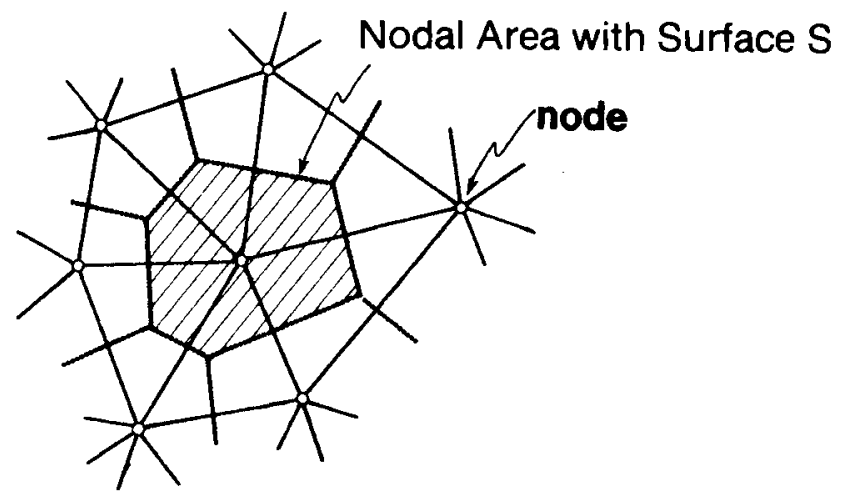

FIGURE B-3. Polygon Geometry (after Thomas 1973) 
Regardless of the approach, the final result is an algebraic equation for each node in the grid system. For the ground-water flow equation posed in terms of head and for a rectangular grid the form of a typical equation is

$$
\begin{aligned}
& B_{i, j} h_{i-1, j}^{n}+D_{i, j} h_{i, j-1}^{n}+E_{i, j} h_{i, j}^{n}+F_{i, j} h_{i, j+1}^{n} \\
& +H_{k, j} h_{i+1, j}^{n} \approx Q_{i, j}^{n-1} .
\end{aligned}
$$

The notation in Equation ( $B-1$ ) refers to the nodal locations shown in Figure $B-2$ where $h$ is the head at the designated node; the explicit definitions of the coefficients $B_{i, j}, D_{i, j}, E_{i, j}, F_{i, j}$ and $H_{i, j}$ are not given here, but may be found, for example, in Freeze and Cherry (1979). The main reason for presenting Equation (B-1) is to demonstrate the forn of the algebraic equation. This equation is for an arbitrary node $(i, j)$ and as may be seen, it has contributions from four adjacent nodes: north $(i, j+1)$, east $(i+1, j)$, south $(i, j-1)$, and west $(i-1, j)$. These are evaluated at the new time level $(n)$ and are related to some known quantity, $Q_{i, j}^{n-1}$, which is computed from information at the old time level $(n-1)$.

Writing an equation similar to (B-1) for each node results in $\mathrm{N}$ equations with $N$ unknown head values to be determined, where $N$ is the total number of nodes. This may be formulated in matrix form and solved using matrix methods. These are discussed in a later section.

A partial history of the use of this approach in solving the groundwater flow equation may be found in Pinder and Bredehoeft (1968) and Remson et al. (1971). Details of this approach applied to petroleum problems similar to ground-water flow and transport problems may be found in Crichlow (1977) and Peaceman (1977).

\section{FINITE ELEMENT METHODS (FEM)}

There are two fundamental problems in calculus: (1) examining the area under a curve, i.e., integration; and (2) examining the tangent of a curve at a point, i.e., differentiation. Both of these concepts were fairly well 
understood by the seventeenth century. For example, Archimedes demonstrated an understanding of integration by deriving his approximation for $\pi$. However, it was not until 1667 that Isaac Barrow (1630-1677), the teacher of Newton, discovered that integration and differentiation are essentially inverse to one another, which is the fundamental theorem of calculus (Allendoerfer and Oakley 1959).

Whereas FDM approximate differential equations by a differential approach, FEM approximate differential equations by an integral approach. Based on the fundamental theorem, one would expect the two methods to be related and should converge to the same solution, but perhaps from different directions.

The FEM actually refer to the numerical method whereby a region is divided into subregions called elements, whose shapes are determined by a set of points called nodes (see Figure B-4). Note that flexibility of elements enables consideration of regions with complex geometry; for example, a water-table aquifer with a meandering river can be outlined with elements fairly accurately.

For transient problems, the time domain may also be approximated using finite elements. In general, however, most studies use finite-difference approximations for the time derivatives.

Triangles were used in the grid shown in Figure B-4, however, several other element shapes may be used. For one-dimensional problems, the elements are lines; for two dimensions, the elements may be either triangles or quadrilaterals; and for three dimensions, they are tetrahedrons or prisms.

The first step in applying the FEM is to derive an integral representation of the partial differential equation. This may be accomplished by several methods; two of the more popular ones include: (1) the method of weighted residuals and (2) the variational method. In the method of weighted residuals (see, for example, Finlayson 1972), one works directly with the differential equation and boundary conditions, whereas in the variational method (see, for example, Zienkiewicz 1971), one uses a functional (a function of a function) related to the differential equation and boundary 


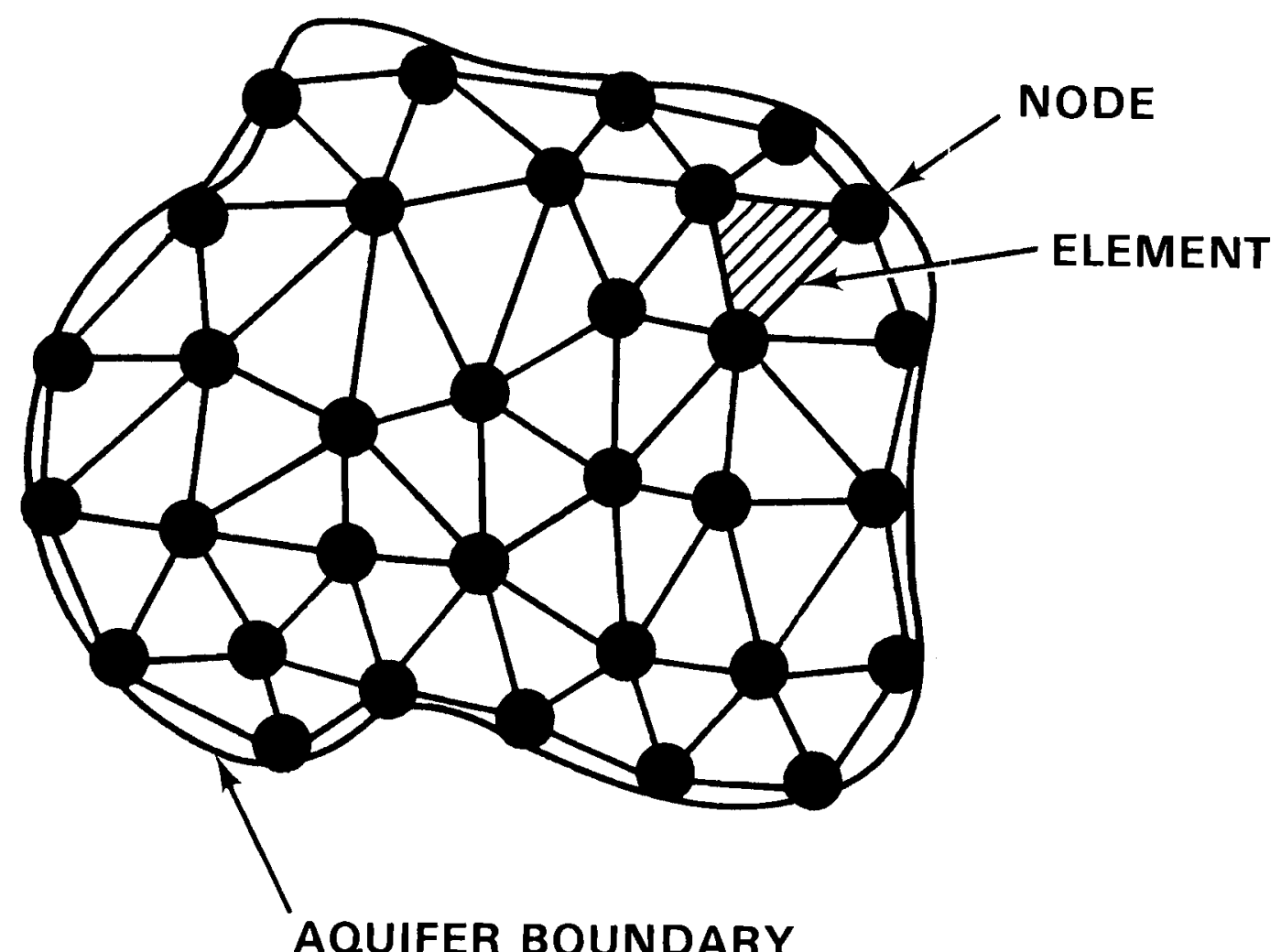

\section{FIGURE B-4. Finite Element Configuration}

Showing Typical Node and Element

(from Faust and Mercer 1980) 
conditions. The mathematics of both of these approaches is fairly straightforward, but not intuitive.

The next step is to approximate the dependent variables (head, concentration or temperature) in terms of interpolation functions. The interpolation functions are called basis functions, and are chosen to satisfy certain mathematical requirements and for ease of computation. Although any system of independent functions can be chosen as the basis function, piecewisecontinuous polynomial sets are often preferred because they are both easily integrated and differentiated. Since the element is usually small, the interpolation function can be sufficiently approximated by a low-order polynomial, for example, linear, quadratic, or cubic. As an example, consider a linear basis function for a triangular element. This basis function describes a plane surface including the values of the dependent variable (head) at the node points in the element. This is illustrated in Figure B-5. For additional information on basis functions, see Desai and Abel (1972) or Zienkiewicz (1971).

Once the basis functions are specified and the grid designed, the integral relationship must be expressed for each element as a function of the coordinates of all node points of the element. Next the values of the integrals are calculated for each element. The values for all elements are combined, including boundary conditions, to yield a system of first-order linear differential equations in time. As previously mentioned, this is approximated using finite-difference techniques to produce a set of algebraic equations. As with finite-difference equations, matrix methods are required for solution.

For additional information on the use of FEM used in ground water, see Verruijt (1970), Remson et a1. (1971), and Pinder and Gray (1977). 


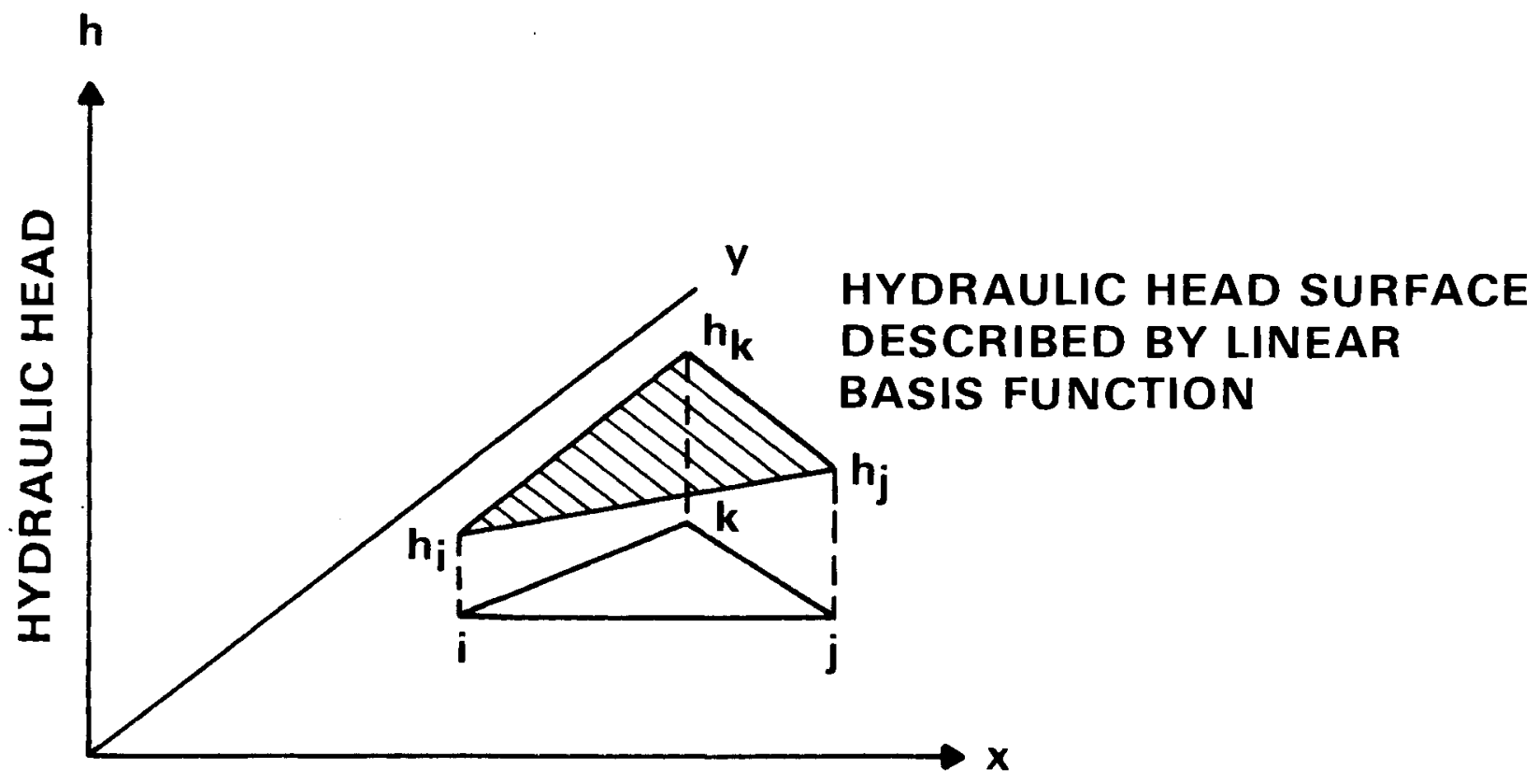

FIGURE B-5. Surface Described by Linear Basis Function for a Triangular Element (from Faust and Mercer 1980) 


\section{DISTRIBUTION}

No. of

Copies

\section{OFFSITE}

A. A. Churm

DOE Patent Division

9800 S. Cass Avenue

Argonne, IL 60439

John J. Brogan

DOE Office of Advanced Conservation Technologies Washington, DC 20585

Arnold Epstein, Project Manager CR-341, Forrestal Building DOE

Washington, DC 20585

B. J. Gallagher

DOE Office of Advanced Conservation Technologies

Washington, DC 20585

T. Levinson

DOE Office of Advanced Conservation Technologies

Washington, DC 20585

G. F. Pezdirtz

DOE Office of Advanced Conservation Technologies

Washington, DC 20585

Veronica $\mathrm{Rabl}$

DOE Office of Advanced Conservation Technologies

Washington, DC 20585

S. Strauch

DOE Office of Advanced Conservation Technologies

Washington, DC 20585
No. of

Copies

J. H. Swisher

DOE Office of Advanced Conservation Technologies

Washington, DC 20585

213 Technical Information Center

Fred J. Molz

School of Engineering

Auburn University

Auburn, AL 36830

Donald Langmuir

Department of Chemistry and Geochemistry

Colorado School of Mines

Golden, C0 80401

Louis Stern

Dames \& Moore

6 Commerce Drive

Cranford, NJ 07016

Walter Hausz

GE-TEMPO

Center for Advanced Studies

816 State Street

P.0. Drawer QQ

Santa Barbara, CA 93102

Charles F. Meyer

GE-TEMPO

Center for Advanced'Studies

816 State Street

P.0. Drawer QQ

Santa Barbara, CA 93102

Chin $\mathrm{Fu}$ Tsang

B1dg 90, Room 1012-H

University of California

Lawrence Berkeley Laboratory

1 Cyclotron Road

Berkeley, CA 94720 
No. of

Copies

Victor E. Hampel

Integrated Information Systems

Computation Dept., L-275

University of California

Lawrence Livermore Laboratory

P.0. Box 808

Livermore, CA 94550

Charles H. Lee

Midwest Research Institute

425 Volker Blvd

Kansas City, MO 64110

Mat Walton

Minnesota Geological Survey

319 15th Avenue S.E.

Minneapolis, MN 55455

National Aeronautics and Space Adminstration Asst Adm for Energy Programs Washington, DC 20546

National Science Foundation Division of Advanced Energy

Research and Technology

Room 1140

1800 G Street, NW

Washington, DC 20550

Director

New York State Energy Research

\& Development Agency

Rockefeller Plaza

Albany, NY 12223

J. F. Martin

Oak Ridge National Laboratory

P.0. Box $Y$

Oak Ridge, TN 37830

Douglas D. Huxtable, Director Energy R\&D

Rocket Research Company

York Center

Redmond, WA 98052
No. of

Copies

L. Radosevich

Sandia Laboratories, Livermore

P.0. Box 969

Livermore, CA 94550

W. G. Wilson

Sandia Laboratories, Livermore

P.0. Box 969

Livermore, CA 94550

Frank Baylin

Solar Enercly Research Institute

1536 Cole Eilvd

Golden, C0 80401

Charles Wymian

Solar Energy Research Institute

1536 Cole Blvd

Golden, CO 80401

Allan Michaels

Solar Thermal Storage Programs

Argonne National Laboratory

Building 362

9700 South Cass Avenue

Argonne, IL 60439

John F. Spencer

Department of Ecology

State of Washington

01 ympia, WA 98504

J. M. Cherne

TRW

One Space Park

Redondo Beach, CA 90278

Will iam Waldrop

Assistant Branch Chief

Water Systerns Development Branch

Division of Water Management

Tennessee Valley Authority

P.0. Drawer $E$

Norris, TN 37828 
No. of

Copies

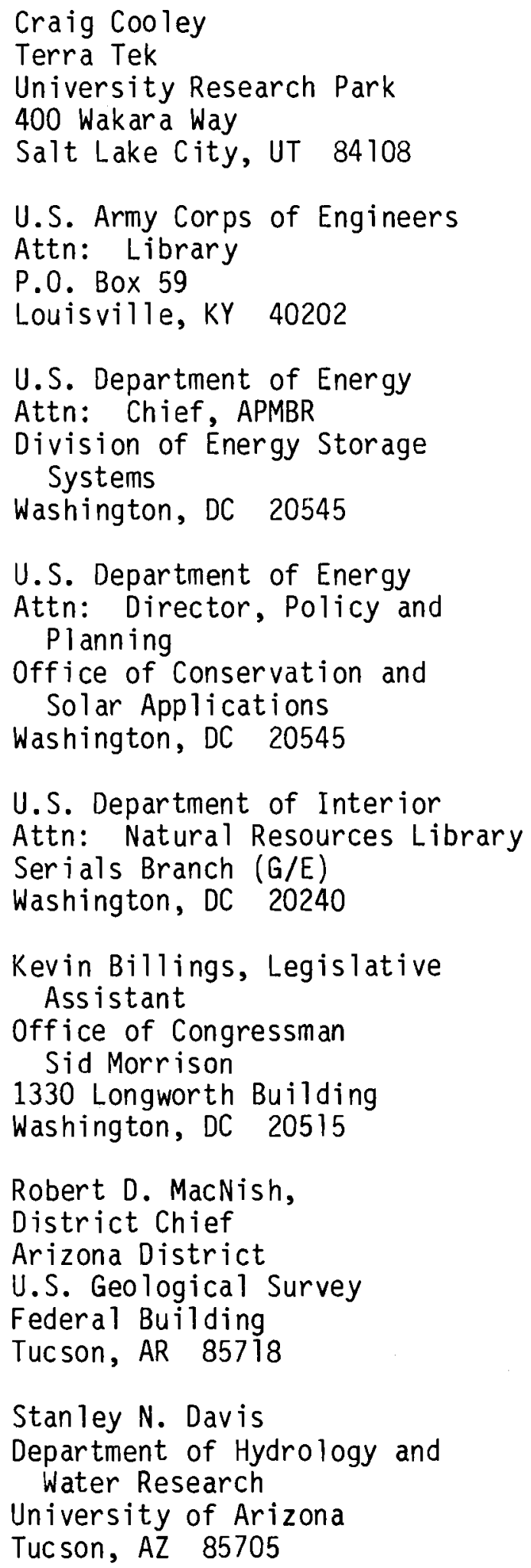

No. of

Copies

\author{
W. E. Soderberg \\ Program Director, ATES \\ University of Minnesota \\ Physical Plant Operations \\ 200 Shops Bldg. \\ 319 15th Avenue S.E. \\ Minneapolis, MN 55455 \\ James W. Crosby III \\ Geology Department \\ Washington State University \\ Pullman, WA 99164 \\ David L. Schreiber, Ph.D., P.E. \\ Consulting Hydraulic Engineer \\ P.0. Box 1087 \\ (c/o The Colony) \\ Coeur d'Alene, ID 83814
}

\section{ONSITE}

DOE Richland Operations Office

H. E. Ransom

57 Pacific Northwest Laboratory

D. Blahnik

C. H. Bloomster

S. M. Brown

D. B. Cear lock

D. E. Deonigi

T. J. Doherty

J. R. Eliason

K. Fox

W. A. Frier

A. F. Gasperino

P. L. Hendr ickson

C. T. Kincaid (5)

L. D. Kannberg

W. W. Laity

W. V. Loscutoff

R. P. Marshall

J. E. Minor

D. A. Myers

J. R. Raymond

R. W. Reilly 
No. of

Copies

W. D. Richmond

L. S. Prater

J. A. Stott lemyre

R. A. Walter

T. L. Willke

G. E. Wukelic

STES Library (20)

Technical Information (5)

Publishing Coordination (2) 